Regularidade analítica para estruturas

de coposto um

\author{
Érik Fernando de Amorim
}





\title{
Regularidade analítica para estruturas de coposto um
}

\author{
Érik Fernando de Amorim
}

Orientador: Prof. Dr. Sérgio Luís Zani

\begin{abstract}
Dissertação apresentada ao Instituto de Ciências Matemáticas e de Computação - ICMC-USP, como parte dos requisitos para obtenção do título de Mestre em Ciências - Matemática. VERSÃO REVISADA
\end{abstract}


Ficha catalográfica elaborada pela Biblioteca Prof. Achille Bassi e Seção Técnica de Informática, ICMC/USP, com os dados fornecidos pelo(a) autor(a)

Amorim, Érik Fernando
Regularidade analítica para estrutura de coposto
um / Érik Fernando Amorim; orientador Sérgio Luís
Zani. -- São Carlos, 2014.
112 p.
Dissertação (Mestrado - Programa de Pós-Graduação
em Matemática) - Instituto de Ciências Matemáticas
e de Computação, Universidade de São Paulo, 2014.
1. Equações diferenciais parciais lineares. 2.
Hipoeliticidade analítica. 3. Sistemas involutivos.
I. Zani, Sérgio Luís, orient. II. Título.




\section{Agradecimentos}

Ao Instituto de Ciências Matemáticas e de Computação da Universidade de São Paulo, por me proporcionar todos os recursos necessários para minha formação matemática e para o desenvolvimento deste trabalho. Ao corpo docente do Instituto; todos têm sua contribuição direta ou indireta em minha educação.

Ao professor Sérgio Luís Zani, por todo o empenho que dedicou ao projeto. Orientador durante os dois anos desse projeto e os três anos de iniciação científica que o precederam, teve papel decisivo no despertar de meu interesse por Análise.

Ao professor Adalberto Bergamasco, que em diversas oportunidades auxiliou na resolução de empecilhos matemáticos que surgiram pelo caminho.

À FAPESP, pelo apoio financeiro. 

Neste trabalho consideramos sistemas de equações diferenciais parciais lineares de primeira ordem, com coeficientes analíticos, definidos em variedades analíticas reais, no caso particular em que seu coposto é igual a um. Demonstramos que esse tipo de sistema admite integrais primeiras locais, e buscamos caracterizar sua hipoelipticidade analítica local e global em termos de propriedades topológicas das mesmas. Também provamos a Fórmula de Aproximação de Baouendi-Trèves. 

In this work we consider systems of first-order linear partial differential equations, with analytic coefficients, defined on real-analytic manifolds, in the special case in which the corank is equal to one. We prove that this type of systems admits local first integrals, and we seek to characterize their local and global analytic hypoellipticity in terms of topological properties of these first integrals. We also prove the Baouendi-Trèves Approximation Formula. 



\section{Introdução}

O presente trabalho está dividido em quatro capítulos, sendo que o primeiro aborda a base da teoria a ser utilizada, os dois seguintes contêm os resultados principais, e o quarto trata do tópico a partir de um ponto de vista moderno.

No primeiro capítulo desenvolvemos as ferramentas básicas da teoria de sistemas involutivos que serão necessárias para a formulação e resolução dos problemas presentes nos capítulos subsequentes. Introduzimos os conceitos de campos de vetores, vetores tangentes e formas diferenciais, permitindo-nos abordar questões de equações diferenciais parciais lineares sobre variedades suaves como questões pertinentes a essa teoria. Demonstramos o Teorema de Frobenius nesse contexto, que posteriormente é empregado na prova da existência de integrais primeiras locais para certos tipos de estruturas involutivas. Estas, por sua vez, desempenham papel fundamental durante o restante do trabalho. Mostramos como empregar mudanças de coordenadas com o propósito de simplificar os sistemas estudados sem perda de generalidade. Também apresentamos nossa demonstração de uma generalização do Princípio de Duhamel para sistemas; trata-se de uma fórmula importante no arsenal de qualquer analista e que no entanto é raramente mencionada nos livros. Finalmente, fechamos o capítulo com uma demonstração do Teorema de Nirenberg, a respeito da existência de integrais primeiras locais quando a estrutura em questão é elíptica.

O segundo capítulo é dedicado ao estudo da hipoelipticidade analítica local, tal como feito em [BT]. O resultado principal afirma que, para uma estrutura involutiva analítica de coposto um definida em uma variedade analítica, a propriedade de hipoelipticidade analítica em um ponto é equivalente à existência de uma integral primeira aberta em torno deste ponto. A demonstração deste fato envolverá o uso do chamado princípio de constância local das soluções da estrutura em questão. Hoje se conhecem versões mais fortes desse princípio, inclusive sem restrições sobre o posto da estrutura, mas a versão e a demonstração que apresentamos são a forma como esse importante resultado foi originalmente descoberto. O primeiro enunciado do principio a ser apresentado aqui, tratando 
de soluções contínuas, envolve um estudo meticuloso das fibras de uma integral primeira em torno de um ponto, e sua demonstração dependerá decisivamente do fato do coposto ser igual a um. O segundo enunciado, a respeito de soluções distribucionais, envolve um estudo da hipoelipticidade parcial dos operadores em questão (após uma mudança de coordenadas conveniente), e para tanto fez-se necessário explorar as propriedades de espaços vetoriais que aqui chamamos de espaços de Sobolev parciais. Trata-se de um conceito que não é estudado em detalhes na bibliografia, e que por isso gerou as maiores dificuldades durante o desenvolvimento deste projeto.

O terceiro capítulo trata novamente de hipoelipticidade analítica, mas agora no caso global, seguindo [BZ]. Dada uma estrutura involutiva analítica de coposto um sobre uma variedade analítica, definimos os chamados conjuntos de nivel, que essencialmente consistem das fibras locais de integrais primeiras coladas umas às outras. O principal resultado caracteriza a hipoelipticidade analítica global da estrutura em termos da existência, em cada conjunto de nível, de um ponto em torno do qual as integrais primeiras sejam abertas. Ressaltamos que essa caracterização é válida apenas sob certas hipóteses topológicas a respeito dos conjuntos de nível, e mencionamos um exemplo em que as hipóteses e a caracterização são violadas.

Finalmente, no quarto capítulo reproduzimos em detalhes uma demonstração da Fórmula de Aproximação de Baouendi-Trèves, baseada em [BCH]. Em linhas gerais, tratase de um teorema que afirma que toda solução suave de uma estrutura localmente integrável pode ser localmente aproximada por polinômios nas integrais primeiras. Este teorema pode ser considerado o resultado mais importante da teoria de sistemas involutivos, sendo empregado para as mais diversas aplicações. O próprio princípio da constância local também é uma consequência simples da aproximação fornecida fórmula. Entretanto, a complexidade do tópico não nos permitiu estudar aproximação de soluções distribucionais por esse método, e por isso foi necessário fazê-lo como no segundo capítulo.

As seguintes convenções notacionais serão utilizadas:

- Se $f: U \subseteq \mathbb{R}^{N} \rightarrow \mathbb{C}$ é uma aplicação diferenciável, $U$ aberto, a derivada parcial de $f$ com relação à coordenada $x_{j}$ será denotada às vezes por $\frac{\partial f}{\partial x_{j}}$, às vezes por $\partial_{x_{j}} f$. Diremos que f é suave se é de classe $\mathrm{C}^{\infty}$ em $\mathrm{U}$.

- Analítico sempre se refere a analítico real, e holomorfo é usado para analítico complexo. Se $\mathrm{U} \subseteq \mathbb{R}^{N}$ é aberto, $\mathrm{C}^{\omega}(\mathrm{U})$ denota o conjunto das funções analíticas em U. Se $\mathrm{V} \subseteq \mathbb{C}^{\mathrm{N}}$ é aberto, $\mathcal{O}(\mathrm{V})$ denota o conjunto das funções holomorfas em $\mathrm{V}$.

- O suporte singular de uma distribuição u, denotado por singsupp u, é o complementar do maior aberto onde $u$ é uma função $C^{\infty}$.

- Se $v_{1}, \ldots, v_{n}$ são elementos de um espaço vetorial, o espaço gerado por eles será denotado por $\operatorname{span}\left\{v_{1}, \ldots, v_{n}\right\}$. 
- $M_{m}(\mathbb{K})$ denota o espaço das matrizes quadradas $m \times m$ com elementos em um corpo $\mathbb{K}$.

- Quando $\gamma:[a, b] \rightarrow X$ é uma curva num espaço topológico, denotamos também por $\gamma$ sua imagem.

- Se p é um ponto de um espaço topológico, dizer que uma certa propriedade vale em torno de $p$ significa que existe uma vizinhança aberta de $p$ onde ela é verdadeira.

- Dizemos que um aberto $A$ de um espaço topológico está compactamente contido em um aberto $B$, e escrevemos $A \subset \subset B$, quando $\bar{A} \subset B$.

- No contexto de variedades, o termo carta local em torno de um ponto $p$ pode se referir a um aberto trivializante $\mathrm{U}$, a um sistema de coordenadas euclidianas locais $x=\left(x_{1}, \ldots, x_{N}\right)$, ou ao par $(U, x)$. 

1 Sistemas Involutivos $\quad 1$

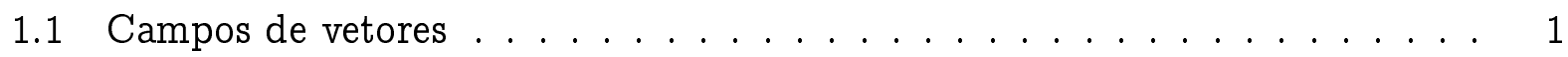

1.2 Vetores tangentes . . . . . . . . . . . . . . 5

1.3 Formas diferenciais . . . . . . . . . . . . . . . . . 8

1.4 Campos e formas reais . . . . . . . . . . . . . . . . 13

1.5 Teorema de Frobenius . . . . . . . . . . . . . . . . . 14

1.6 Estruturas holomorfas . . . . . . . . . . . . . . . 18

1.7 Conjunto característico . . . . . . . . . . . . . . . 19

1.8 Tipos de estruturas . . . . . . . . . . . . . . . . . 21

1.9 Estruturas analíticas . . . . . . . . . . . . . . . . 24

1.10 Geradores locais . . . . . . . . . . . . . . . . 25

1.11 Estruturas localmente integráveis . . . . . . . . . . . . . . . 29

1.12 Teorema de Nirenberg . . . . . . . . . . . . . . . . 40

2 Regularidade Analítica Local $\quad 45$

2.1 Princípio da constância local . . . . . . . . . . . . . . . 46

2.2 Soluções distribucionais . . . . . . . . . . . . . . . . . . 57

2.3 Consequências para hipoelipticidade . . . . . . . . . . . . . . . 74

2.4 Hipoelipticidade parcial das soluções . . . . . . . . . . . . . . 76

$\begin{array}{lll}3 & \text { Regularidade Analítica Global } & 79\end{array}$ 
3.1 Conjuntos de nível . . . . . . . . . . . . . . . . . 79

3.2 Hipoelipticidade analítica global . . . . . . . . . . . . . . 84

4 Teorema de Aproximação de Baouendi-Trèves 


\begin{tabular}{|l|c|}
\cline { 2 - 2 } & CAPÍtulO \\
\cline { 2 - 2 } & 1 \\
\hline
\end{tabular}

\section{Sistemas Involutivos}

Sistemas involutivos de campos vetoriais complexos são objetos abstratos definidos em uma variedade diferenciável, caracterizados por propriedades que os tornam generalizações de operadores diferenciais parciais lineares homogêneos de primeira ordem, o que nos permite estudar sistemas destes últimos definidos sobre variedades diferenciáveis.

Ao longo deste capítulo, $\Omega$ representará uma variedade $N$-dimensional, $N=1,2, \ldots$

\subsection{Campos de vetores}

Na Geometria, um campo de vetores suave em $\Omega$ é uma aplicação $L: \Omega \rightarrow T \Omega$ de classe $C^{\infty}$ que a cada ponto $p \in \Omega$ associa um vetor $L p \in T_{p} \Omega$ (aqui $T \Omega$ representa o fibrado tangente da variedade, e $\mathrm{T}_{\mathrm{p}} \Omega$ é o espaço tangente em $\mathrm{p}$ ). Entretanto, tendo-se em vista aplicar a teoria de sistemas involutivos às equações diferenciais parciais, é mais conveniente formalizar a seguinte definição:

Definição 1.1. Um campo de vetores (suave, complexo) em $\Omega$ é uma aplicação $\mathbb{C}$-linear

$$
\mathrm{L}: \mathrm{C}^{\infty}(\Omega) \rightarrow \mathrm{C}^{\infty}(\Omega)
$$

(onde $\mathrm{C}^{\infty}(\Omega)$ é o $\mathbb{C}$-módulo das funções suaves de $\Omega$ a valores complexos) que satisfaz a Regra de Leibniz:

$$
L(f g)=f L g+g L f \quad, \quad f, g \in C^{\infty}(\Omega)
$$

Denota-se o conjunto dos campos de vetores de $\Omega$ por $X(\Omega)$. 
O exemplo de campos de vetores mais trivial, e o que nos será mais importante, é o de um operador diferencial parcial linear homogêneo de primeira ordem, a coeficientes $C^{\infty}$.

Segue da Regra de Leibniz que se $f$ é uma função constante então Lf $=0$ para todo campo L. De fato, por $\mathbb{C}$-linearidade basta vermos que $L 1=0$, e isto é consequência de $\mathrm{L} 1=\mathrm{L}(1 \cdot 1)=1(\mathrm{~L} 1)+(\mathrm{L} 1) 1=2(\mathrm{~L} 1)$. Esta propriedade será usada para demonstrarmos:

Proposição 1.2. Se $f \in C^{\infty}(\Omega)$ e $L \in X(\Omega)$, então supp $L f \subseteq \operatorname{supp} f$.

O símbolo supp indica o suporte de uma função, isto é, o fecho do conjunto onde ela não se anula.

Demonstração: Deve-se provar que se $f$ se anula em um aberto $V$ então Lf também se anula ali. Dado $p \in V$, seja $g \in C^{\infty}(\Omega)$ tal que $f=(1-g) f$ e $g(p)=1$. Uma tal função realmente existe: basta escolher um aberto $\mathrm{U}$, com $p \in \mathrm{U} \subset \subset \mathrm{V}$, e uma função suave $\phi$ com suporte em $U$ tal que $\phi(p)=1$, e então definir a função (suave) $g=\phi$ em $U$ e $g \equiv 0$ em $\Omega \backslash U$. Dessa forma, $f=(1-g) f$ em $V$ porque ambos os membros são nulos, e a mesma igualdade vale fora de $\mathrm{V}$ porque ali $1-\mathrm{g} \equiv 1$. Temos portanto

$$
\operatorname{Lf}(p)=L((1-g) f)(p)=(1-g(p)) \operatorname{Lf}(p)+f(p) L(1-g)(p)=0
$$

pois $1-g(p)=f(p)=0$.

Uma consequência deste resultado e da linearidade dos campos é que, assim como é o caso com operadores diferenciais, o valor de Lf num ponto só depende dos valores de $f$ em qualquer vizinhança aberta desse ponto. Com esta propriedade, mostra-se a boa-definição do conceito de restrição de um campo a um aberto: se $U \subseteq \Omega$ é aberto (logo é uma variedade $\mathrm{N}$-dimensional) e $\mathrm{L} \in \mathcal{X}(\Omega)$, define-se o campo $\left.\mathrm{L}\right|_{\mathrm{u}} \in \mathcal{X}(\mathrm{U})$ por

$$
\left.\mathrm{L}\right|_{\mathrm{u}} f(p)=\mathrm{L} \tilde{f}(p) \quad, \quad f \in C^{\infty}(\mathrm{U}) \quad, \quad p \in U
$$

onde $\tilde{f} \in C^{\infty}(\Omega)$ é qualquer função que coincide com $f$ perto de $p$, construída, por exemplo, através de funções de corte. Dessa forma, o diagrama abaixo torna-se comutativo (setas verticais indicam a restrição usual de funções):

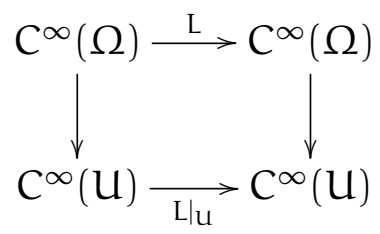

Definição 1.3. Uma solução de um campo $\mathrm{L}$ em um aberto $\mathrm{U} \subseteq \Omega$ é uma função suave $\mathrm{u} \in \mathrm{C}^{\infty}(\mathrm{U})$ tal que $\left.\mathrm{L}\right|_{\mathrm{u}}(\mathrm{u})=0$.

Duas operações importantes com campos são o produto por funções, que transforma $\chi(\Omega)$ em um $\mathrm{C}^{\infty}(\Omega)$-módulo, e o colchete de Lie. Mais precisamente, dados $\mathrm{g} \in \mathrm{C}^{\infty}(\Omega)$ 
e $L, M \in X(\Omega)$, definimos $g L \in X(\Omega)$ e $[L, M] \in X(\Omega)$ por

$$
(g L) f=g \cdot(L f) \quad, \quad[L, M] f=L(M f)-M(L f) \quad, \quad f \in C^{\infty}(\Omega)
$$

Verificar que realmente se trata de campos requer apenas cálculos diretos. Por exemplo, a Regra de Leibniz vale para o colchete de Lie:

$$
\begin{aligned}
{[L, M](f g)=} & L(M(f g))-M(L(f g))=L(f M g+g M f)-M(f L g+g L f) \\
= & f(L(M g))+(L f)(M g)+g(L(M f))+(L g)(M f)- \\
& \quad-f(M(L g))-(M f)(L g)-g(M(L f))-(M g)(L f) \\
= & f([L, M] g)+g([L, M] f)
\end{aligned}
$$

Observação 1.4. Assim como qualquer objeto definido em uma variedade, também os campos de vetores têm uma forma padrão de representação em coordenadas locais. Seja $p \in \Omega$ e seja ( $U, x)$ uma carta local, isto é, $U \subseteq \Omega$ é aberto, $p \in U$, e $x=\left(x_{1}, \ldots, x_{N}\right): U \rightarrow$ $\mathbb{R}^{N}$ é um difeomorfismo suave. As $x_{j}$ são as chamadas funções coordenadas locais. Denote por $a=\left(a_{1}, \ldots, a_{N}\right) \circ$ ponto $x(p) \in \mathbb{R}^{N}$. Então $f \circ x^{-1}: x(U) \rightarrow \mathbb{C}$ é uma função suave, e assim existe um aberto $V \subseteq \mathbb{R}^{N}$, com $a \in V$, e existem funções suaves $h_{1}, \ldots, h_{N}: V \rightarrow \mathbb{C}$ tais que

$$
\left(f \circ x^{-1}\right)(y)=\left(f \circ x^{-1}\right)(a)+\sum_{j=1}^{N} h_{j}(y)\left(y_{j}-a_{j}\right) \quad, \quad y \in V
$$

De fato, esta é uma aplicação padrão do teorema fundamental do cálculo. As funções $h_{j}$ são dadas por

$$
h_{j}(y)=\int_{0}^{1} \frac{\partial\left(f \circ x^{-1}\right)}{\partial y_{j}}\left(a_{1}+t\left(y_{1}-a_{1}\right), \ldots, a_{N}+t\left(y_{N}-a_{N}\right)\right) d t
$$

Transferindo os pontos a $U \subseteq \Omega$ através do difeomorfismo $x$, obtemos de (1.5) acima que

$$
f(q)=f(p)+\sum_{j=1}^{N}\left(h_{j} \circ x\right)(q)\left(x_{j}(q)-x_{j}(p)\right), q \in x^{-1}(V)
$$

Logo, se $\mathrm{L} \in \mathcal{X}(\Omega)$ :

$$
\operatorname{Lf}(q)=0+\sum_{j=1}^{N}\left(L\left(h_{j} \circ x\right)(q)\left(x_{j}(q)-x_{j}(p)\right)+\left(h_{j} \circ x\right)(q) L x_{j}(q)\right)
$$

Finalmente, fazendo $q=p$ :

$$
\operatorname{Lf}(p)=\sum_{j=1}^{N}\left(h_{j} \circ x\right)(p) \operatorname{Lx}(p)=\sum_{j=1}^{N} \frac{\partial\left(f \circ x^{-1}\right)}{\partial y_{j}}(x(p)) \operatorname{Lx}(p)
$$

As $\mathrm{N}$ aplicações

$$
\mathrm{C}^{\infty}(\mathrm{U}) \ni \mathrm{f} \mapsto \frac{\partial\left(\mathrm{f} \circ x^{-1}\right)}{\partial y_{j}} \circ x \in \mathrm{C}^{\infty}(\mathrm{U}), \quad j=1, \ldots, N
$$


são campos de vetores de $U$, denotados por $\frac{\partial}{\partial x_{j}}$ ou simplesmente $\partial_{x_{j}}$. É simples ver, aplicando-os separadamente a funções que dependem apenas de cada variável $y_{j}$, que esses campos são linearmente independentes sobre o anel $\mathrm{C}^{\infty}(\mathrm{U})$. Utilizando-os, podemos escrever a equação (1.7) acima como

$$
L=\sum_{j=1}^{N}\left(L x_{j}\right) \frac{\partial}{\partial x_{j}}
$$

Logo $\left\{\partial_{x_{j}} ; j=1, \ldots, N\right\}$ constitui uma base para o $\mathrm{C}^{\infty}(\mathrm{U})$-módulo $X(\mathrm{U})$.

Teorema 1.8. (Regra da Cadeia) Sejam L um campo de vetores sobre $\Omega$ e u $\in$ $\mathrm{C}^{\infty}(\Omega)$. Se f é diferenciável em um aberto contido em $\mathrm{u}(\Omega) \subseteq \mathbb{C}$ (considerando-se $f$ como função definida em um aberto de $\mathbb{R}^{2}$ ), então

$$
\mathrm{L}(\mathrm{f} \circ \mathrm{u})(\mathrm{p})=\frac{\partial \mathrm{f}}{\partial \zeta}(\mathrm{u}(\mathrm{p})) \mathrm{Lu}(\mathrm{p})+\frac{\partial \mathrm{f}}{\partial \bar{\zeta}}(\mathrm{u}(\mathrm{p})) \mathrm{L} \bar{u}(\mathrm{p})
$$

Em particular, se u é solução de L e f é holomorfa, então fou também é solução de L.

Demonstração: Podemos provar o resultado localmente, e portanto assumir que L é uma combinação $C^{\infty}$-linear de derivadas $\partial_{x_{j}}$. Mais do que isso, dada a $C^{\infty}$-linearidade da equação a ser provada, não há perda de generalidade em assumir que $L=\partial_{x_{j}}$ para algum $j=1, \ldots, N$. Identifique a imagem de $u$ em $\mathbb{C}$ com um subconjunto de $\mathbb{R}^{2}$, mas com "coordenadas" $(\zeta, \bar{\zeta})$. A rigor, não se trata de coordenadas, pois $\zeta$ e $\bar{\zeta}$ não são números reais, mas todos os procedimentos padrões do Cálculo, como por exemplo a regra da cadeia usual, podem ser aplicados a $\left(\mathbb{R}^{2},(\zeta, \bar{\zeta})\right)$ como se fossem. Dessa forma as funções coordenadas de $u$ são $u$ e $\bar{u}$. Identifique também a imagem de $f$ em $\mathbb{C}$ com $\mathbb{R}^{2}$, com as coordenadas usuais $(\mathfrak{R} \zeta, \mathfrak{I} \zeta$ ); portanto as funções coordenadas de $f$ são $\mathfrak{R} f$ e If. Nessas condições, a regra da cadeia usual afirma que $\partial_{x_{j}}(f \circ u)$ é dado pelo vetor $\left(\nu_{1}, v_{2}\right)$, onde

$$
\begin{aligned}
& v_{1}=\left(\frac{\partial \mathfrak{R} f}{\partial \zeta} \circ u\right) \frac{\partial u}{\partial x_{j}}+\left(\frac{\partial \Re \mathfrak{R}}{\partial \bar{\zeta}} \circ u\right) \frac{\partial \bar{u}}{\partial x_{j}} \\
& v_{2}=\left(\frac{\partial \mathfrak{I} f}{\partial \zeta} \circ u\right) \frac{\partial u}{\partial x_{j}}+\left(\frac{\partial \mathfrak{I} f}{\partial \bar{\zeta}} \circ u\right) \frac{\partial \bar{u}}{\partial x_{j}}
\end{aligned}
$$

De acordo com nossa última identificação, $\left(v_{1}, v_{2}\right)$ corresponde ao número complexo $v_{1}+\mathfrak{i} v_{2}$, isto é, ao número

$$
\begin{aligned}
& \left(\frac{\partial \Re f}{\partial \zeta} \circ u\right) \frac{\partial u}{\partial x_{j}}+\left(\frac{\partial \Re f}{\partial \bar{\zeta}} \circ u\right) \frac{\partial \bar{u}}{\partial x_{j}}+ \\
& +i\left(\left(\frac{\partial I f}{\partial \zeta} \circ u\right) \frac{\partial u}{\partial x_{j}}+\left(\frac{\partial \Im f}{\partial \bar{\zeta}} \circ u\right) \frac{\partial \bar{u}}{\partial x_{j}}\right)= \\
& =\left(\frac{\partial f}{\partial \zeta} \circ u\right) \frac{\partial u}{\partial x_{j}}+\left(\frac{\partial f}{\partial \bar{\zeta}} \circ u\right) \frac{\partial \bar{u}}{\partial x_{j}}
\end{aligned}
$$




\subsection{Vetores tangentes}

Assim como foi feito com os campos, é mais conveniente definir vetor tangente não como na Geometria, mas como uma aplicação que age em funções, ou neste caso em germes de funções. Um germe de funções suaves em um ponto $p \in \Omega$ é uma classe de equivalência da relação

$$
f \sim g \Longleftrightarrow f=g \text { em algum aberto } U \ni p \quad, \quad f, g \in C^{\infty}(\Omega)
$$

O germe de uma função $f$ será denotado também por f. O espaço dos germes em $p$ é um anel que denotaremos por $C^{\infty}(p)$.

Definição 1.9. Um vetor tangente (complexo) a $\Omega$ em $p \in \Omega$ é uma aplicação $\mathbb{C}$-linear

$$
v: \mathbb{C}^{\infty}(p) \rightarrow \mathbb{C}
$$

tal que

$$
v(f g)=f(p) v(g)+g(p) v(f) \quad, \quad f, g \in C^{\infty}(p)
$$

O espaço tangente (complexo) a $\Omega$ em $p$, denotado por $\mathbb{C T}_{p} \Omega$, é o $\mathbb{C}$-espaço vetorial dos vetores tangentes em $p$.

Dado um campo de vetores $L$ segundo a noção geométrica, podemos falar em seu valor num ponto $p \in \Omega$ : é o vetor tangente $L(p)$. A analogia com os campos como foram definidos aqui traduz esse conceito no seguinte: a localização de L em $p$ é o vetor tangente $\mathrm{L}_{\mathrm{p}}$ definido por

$$
\mathrm{L}_{p}(f)=L f(p) \quad, \quad f \in C^{\infty}(p)
$$

Essa operação está bem definida para germes porque o valor de Lf em $p$ depende de $f$ apenas em alguma vizinhança aberta de $p$. Também é claramente $C^{\infty}(\Omega)$-linear, no seguinte sentido:

$$
\left(L_{1}+f L_{2}\right)_{p}=\left(L_{1}\right)_{p}+f(p)\left(L_{2}\right)_{p} \quad, \quad L_{1}, L_{2} \in X(\Omega), f \in C^{\infty}(\Omega)
$$

Utilizando-se a representação local de campos, é simples provar o seguinte resultado:

Proposição 1.10. $\mathbb{C} T_{p} \Omega=\left\{L_{p} ; L \in X(\Omega)\right\}$ para todo $p \in \Omega$.

Se $(U, x)$ é uma carta local de $\Omega$ em torno de $p$, podemos usar a equação (1.6) acima e obter a representação local de um vetor tangente. De fato, aquela equação dizia que existe um aberto $\mathrm{V} \subseteq \mathrm{U}$, contendo $p$, onde qualquer $f \in \mathrm{C}^{\infty}(\Omega)$ é dada por

$$
f(q)=f(p)+\sum_{j=1}^{N}\left(h_{j} \circ x\right)(q)\left(x_{j}(q)-x_{j}(p)\right) \quad, \quad q \in x^{-1}(V)
$$


com as $h_{j}$ definidas como antes. Logo, se $v \in \mathbb{C} T_{p} \Omega$, temos

$$
\begin{aligned}
v(f) & =0+\sum_{j=1}^{N} v\left\{\left(h_{j} \circ x\right)(\cdot)\left(x_{j}(\cdot)-x_{j}(p)\right)\right\} \\
& =\sum_{j=1}^{N} v\left\{\partial_{x_{j}} f(\cdot)\left(x_{j}(\cdot)-x_{j}(p)\right)\right\} \\
& =\sum_{j=1}^{N}\left(\partial_{x_{j}} f(p) v\left(x_{j}\right)+\left(x_{j}(p)-x_{j}(p)\right) v\left(\partial_{x_{j}} f\right)\right) \\
& =\sum_{j=1}^{N} \partial_{x_{j}} f(p) v\left(x_{j}\right)=\sum_{j=1}^{N} v\left(x_{j}\right)\left(\partial_{x_{j}}\right)_{p}(f)
\end{aligned}
$$

Como f é arbitrária, concluímos que

$$
v=\sum_{j=1}^{N} v\left(x_{j}\right)\left(\frac{\partial}{\partial x_{j}}\right)_{q}
$$

Assim, a base canônica para $\mathbb{C T}_{\mathrm{p}} \mathrm{U}$ sobre $\mathbb{C}$ é $\left\{\left(\partial_{x_{1}}\right)_{p}, \ldots,\left(\partial_{x_{N}}\right)_{p}\right\}$.

O fibrado tangente (complexificado) de $\Omega$ é a reunião disjunta dos espaços tangentes a cada ponto de $\Omega$, denotado por $\mathbb{C} T \Omega$. Na Geometria, um subfibrado (real) deste fibrado vetorial consiste em uma reunião disjunta localmente trivial de subespaços de cada espaço tangente, todos com mesma dimensão real $n$. A trivialidade local significa que, em torno de cada ponto da variedade, existe um aberto $\mathrm{U}$ tal que o subfibrado restrito a $\mathrm{U}$ é difeomorfo a $\mathrm{U} \times \mathbb{R}^{\mathrm{n}}$. Na teoria de sistemas involutivos, este conceito se transforma no seguinte:

Definição 1.11. Um subfibrado vetorial (complexo) de $\mathbb{C} T \Omega$ de posto $\mathbf{n}$ e coposto $\mathbf{N}-\mathbf{n}$ é uma reunião disjunta

$$
\nu=\bigcup_{p \in \Omega} \nu_{p} \subseteq \mathbb{C T} \Omega
$$

onde cada $\mathcal{V}_{\mathrm{p}}$ é um subespaço de $\mathbb{C T}_{\mathrm{p}} \Omega$ de dimensão $\mathrm{n}$, satisfazendo a condição de trivialidade local: para cada $\mathrm{p} \in \Omega$, existem um aberto $\mathrm{U} \ni \mathrm{p}$ e campos $\mathrm{L}_{1}, \ldots, \mathrm{L}_{\mathrm{n}} \in$ $X(\mathrm{U})$ tais que, para todo $\mathrm{q} \in \mathrm{U}, \nu_{\mathrm{q}}$ é gerado pelos vetores tangentes $\left(\mathrm{L}_{1}\right)_{\mathrm{q}}, \ldots,\left(\mathrm{L}_{n}\right)_{\mathrm{q}}$. Também podemos nos referir a $V$ como um subfibrado tangente. Diz-se que os campos $\mathrm{L}_{\mathrm{j}}$ geram $\mathcal{V}$ em torno de $\mathbf{p}$. O espaço vetorial $\mathcal{V}_{\mathrm{p}}$ é dito a fibra de $\mathcal{V}$ em $\mathrm{p}$. Uma seção do subfibrado $\mathcal{V}$ sobre um aberto $\mathrm{W} \subseteq \Omega$ é um campo de vetores $\mathrm{L} \in X(\mathrm{~W})$ tal que $\mathrm{L}_{\mathrm{p}} \in \mathcal{V}_{\mathrm{p}}$ para cada $\mathrm{p} \in \mathrm{W}$.

Nosso principal objeto de estudo neste projeto serão os subfibrados vetoriais. Mas como o colchete de Lie terá papel fundamental nos teoremas que virão, é necessário nos restringirmos aos subfibrados que são num certo sentido fechados segundo essa operação. Isto é o que diz a próxima definição. 
Definição 1.12. Uma estrutura involutiva sobre $\Omega$ de posto $\mathbf{n}$, também chamada estrutura formalmente integrável, é um subfibrado $\mathcal{V}$ de posto $\mathrm{n}$ satisfazendo a condição involutiva de Frobenius: se $\mathrm{W} \subseteq \Omega$ é aberto e $\mathrm{L}, \mathrm{M} \in X(\mathrm{~W})$ são seções de $\mathcal{V}$ sobre $\mathrm{W}$, então $[\mathrm{L}, \mathrm{M}]$ também é seção de $\mathcal{V}$ sobre $\mathrm{W}$.

Uma solução clássica da estrutura involutiva $\mathcal{V}$ sobre um aberto $W \subseteq \Omega$ é uma função suave $u \in C^{\infty}(W)$ tal que $L u=0$ para toda seção $L$ de $\mathcal{V}$ sobre $W$. Pode-se denotar este fato escrevendo-se que $V u=0$ em $W$. Uma solução generalizada de $V$ sobre $W$ é uma distribuição $u \in \mathcal{D}^{\prime}(W)$ tal que $L u=0$ para toda seção $L$ de $\mathcal{V}$ sobre $W$. Não abordaremos distribuições em variedades neste trabalho, mas, ao longo do mesmo, encontraremos a necessidade de buscar por soluções generalizadas de estruturas involutivas. Entretanto, esses casos sempre serão lidados sobre o espaço euclidiano, e ali os campos de vetores não passam de operadores diferenciais parciais lineares, e nesse contexto a expressão Lu tem sentido para uma distribuição $u$.

Observe que, caso uma estrutura $\mathcal{V}$ seja gerada por campos $L_{1}, \ldots, L_{n}$, então $\mathcal{V}$ será involutiva se e somente se cada $\left[L_{j}, L_{k}\right]$ for uma seção de $\nu$ sobre $\Omega$, e uma função suave $u \in C^{\infty}(W)$ será uma solução de $\mathcal{V}$ se e somente se $L_{j} u=0$ em $W$ para cada $j=1, \ldots, n$.

Teorema 1.13. Se $V$ é uma estrutura involutiva de posto $\mathrm{n}$ e se os campos $\mathrm{L}_{1}, \ldots, \mathrm{L}_{n}$ geram $\mathcal{V}$ em torno de $p \in \Omega$, então existem funções $\lambda_{j k}^{l} \in C^{\infty}(\Omega), j, k, l=1, \ldots, n$, tais que, em torno de $p$, vale

$$
\left[\mathrm{L}_{j}, \mathrm{~L}_{\mathrm{k}}\right]=\sum_{\mathrm{l}=1}^{\mathrm{n}} \lambda_{j k}^{l} \mathrm{~L}_{l} \quad, \quad j, k=1, \ldots, \mathrm{n}
$$

Demonstração: Por involutividade, como perto de $p$ a estrutura é gerada pelos $L_{j}$, temos que, para cada q suficientemente próximo a $p$, existem $\lambda_{j k}^{v}(q) \in \mathbb{C}, j, k, v=1, \ldots, n$, tais que

$$
\left[\mathrm{L}_{j}, \mathrm{~L}_{\mathrm{k}}\right]_{\mathrm{q}}=\sum_{\nu=1}^{\mathrm{n}} \lambda_{j k}^{v}(\mathrm{q})\left(\mathrm{L}_{v}\right)_{\mathrm{q}}
$$

O trabalho agora é provar que as funções $\lambda_{j k}^{v}$ assim definidas são suaves, porque se isto estiver provado então teremos, para todo q próximo a $p$ e toda $f \in C^{\infty}(\Omega)$,

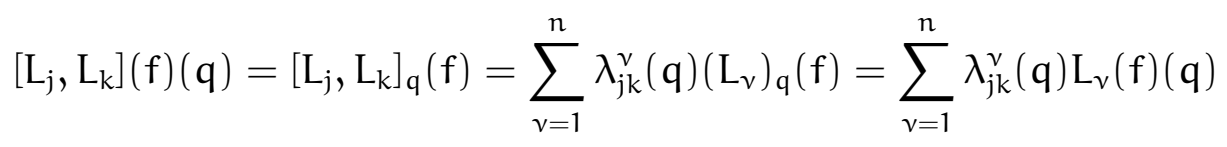

Se lembrarmos que as funções $\lambda_{j k}^{v}$ podem ser estendidas a $\Omega$ com funções de corte, 0 teorema estará provado.

Diminuindo se necessário a vizinhança de $p$ considerada, tome-a como uma carta local U. Existem campos $\partial_{x_{1}}, \ldots, \partial_{x_{N}}$ que geram $\mathcal{V}$ sobre $U$, e então existem $a_{j k} \in C^{\infty}(U)$, 
$j=1 \ldots, n, k=1 \ldots, N$ tais que

$$
L_{j}=\sum_{k=1}^{n} a_{j k} \frac{\partial}{\partial x_{k}} \quad, \quad j=1, \ldots, n
$$

Note que as funções $a_{j k}$ são dadas por

$$
a_{j k}(q)=\sum_{m=1}^{N} a_{j m}(q) \frac{\partial x_{k}}{\partial x_{m}}=L_{j}\left(x_{k}\right)(q)=\left(L_{j}\right)_{q}\left(x_{k}\right)
$$

Como o posto da estrutura é $n$, o posto da matriz $\left(a_{j k}(p) ; 1 \leq j \leq n, 1 \leq k \leq N\right)$ é $n$. Para facilitar a notação, reordene as coordenadas $x_{k}$ de forma que a matriz $\left(a_{j k}(p) ; 1 \leq\right.$ $j \leq n, 1 \leq k \leq n)$ seja invertível. Então

$$
A(q)=\left(a_{j k}(q) ; 1 \leq j \leq n, 1 \leq k \leq n\right)
$$

permanece invertível, por continuidade, para $\mathrm{q}$ em um aberto $\mathrm{V} \subseteq \mathrm{U}$ em torno de $\mathrm{p}$, e os elementos de $A^{-1}(q)$ são funções suaves, de acordo com o algoritmo da inversão de matrizes.

Para cada $q \in V$ e cada $j, k=1, \ldots, n$, defina o vetor

$$
V_{j k}(q)=\left(\lambda_{j k}^{1}(q), \ldots, \lambda_{j k}^{n}(q)\right)
$$

Portanto,

$$
\left[L_{j}, L_{k}\right]\left(x_{l}\right)(q)=\left[L_{j}, L_{k}\right]_{q}\left(x_{l}\right)=\sum_{v=1}^{n} \lambda_{j k}^{v}(q)\left(L_{v}\right)_{q}\left(x_{l}\right)=\sum_{v=1}^{n} \lambda_{j k}^{v}(q) a_{v l}(q)
$$

Isso prova que o vetor $V_{j k}(q) A(q)$ é igual a

$$
\left(\left[\mathrm{L}_{j}, \mathrm{~L}_{k}\right]\left(\mathrm{x}_{1}\right)(\mathrm{q}), \ldots,\left[\mathrm{L}_{\mathrm{j}}, \mathrm{L}_{\mathrm{k}}\right]\left(\mathrm{x}_{\mathrm{n}}\right)(\mathrm{q})\right)
$$

Em particular seus elementos são funções suaves de q. Logo, os elementos do vetor

$$
\left(\lambda_{j k}^{1}(q), \ldots, \lambda_{j k}^{n}(q)\right)=V_{j k}(q)=\left(V_{j k}(q) A(q)\right) A^{-1}(q)
$$

são funções suaves de q, como queríamos provar.

\subsection{Formas diferenciais}

O que nos será útil é mais precisamente o conceito geométrico de 1-formas diferenciais:

Definição 1.14. Uma (1-)forma diferencial em $\Omega$ é um elemento do dual $\Lambda^{1}(\Omega)$ do $\mathrm{C}^{\infty}(\Omega)$-módulo $X(\Omega)$, isto é, uma aplicação $\mathrm{C}^{\infty}(\Omega)$-linear

$$
\omega: X(\Omega) \rightarrow C^{\infty}(\Omega)
$$


Proposição 1.15. Se $\mathrm{L} \in X(\Omega)$ é o campo nulo em um aberto $\mathrm{U} \subseteq \Omega$, então $\omega(\mathrm{L})=0$ em U para toda $\omega \in \Lambda^{1}(\Omega)$.

Demonstração: Seja $p \in U$. Obtenha $g \in C^{\infty}(\Omega)$ que vale 1 em $p$ e 0 fora de $\mathrm{U}$; logo $\mathrm{L}=(1-\mathrm{g}) \mathrm{L}$ em $\Omega$, e tem-se

$$
\omega(\mathrm{L})=\omega((1-g) \mathrm{L})=(1-\mathrm{g}) \omega(\mathrm{L})
$$

que vale 0 em $p$.

Como consequência, prova-se a boa-definição do conceito de restrição de uma forma a um aberto: dados uma forma $\omega \in \Lambda^{1}(\Omega)$ e um aberto $U \subseteq \Omega$, pode-se definir $\left.\omega\right|_{u} \in \Lambda^{1}(U)$ de forma que o diagrama a seguir comute (as setas verticais indicam a restrição de campos e de funções suaves):

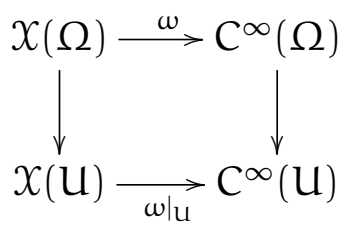

De fato, dados $M \in X(U)$ e $p \in U$, queremos definir $\left.\omega\right|_{u}(M)(p)$. Tome $g \in C_{c}^{\infty}(\Omega)$ tal que $\mathrm{g} \equiv 1$ perto de $\mathrm{p}$, e defina o campo $\tilde{M} \in X(\Omega)$ por

$$
\tilde{M}(f)=M(g f) \quad, \quad f \in C^{\infty}(\Omega)
$$

É simples mostrar que $M$ é de fato um campo de vetores - isto é verdade para qualquer $\mathrm{g} \in \mathrm{C}^{\infty}$. Além disso, $\mathrm{M}=\tilde{\mathrm{M}}$ em uma vizinhança aberta de $\mathrm{p}$. Portanto, a forma diferencial

$$
\left.\omega\right|_{\mathrm{u}}(M)(p)=\omega(\tilde{M})(p)
$$

está bem definida, e se $L \in X(\Omega)$, então

$$
\left.\omega\right|_{\mathrm{u}}\left(\left.\mathrm{L}\right|_{\mathrm{u}}\right)(\mathrm{p})=\omega\left(\left(\left.\mathrm{L}\right|_{\mathrm{U}}\right)^{\sim}\right)(\mathrm{p})=\omega(\mathrm{L})(\mathrm{p})
$$

Isto é, o diagrama acima comuta.

Proposição 1.16. Se $\mathrm{L} \in \mathcal{X}(\Omega)$ é tal que $\mathrm{L}_{\mathrm{p}}=0$ para algum $\mathrm{p} \in \Omega$, então $\omega(\mathrm{L})(\mathrm{p})=0$ para toda $\omega \in \Lambda^{1}(\Omega)$.

Demonstração: Escolha coordenadas locais $x_{1}, \ldots, x_{N}$. Temos $L=\sum_{j=1}^{N} L x_{j} \frac{\partial}{\partial x_{j}}$, e aplicandose $\omega$ :

$$
\omega(L)=\sum_{j=1}^{N}\left(L x_{j}\right) \omega\left(\frac{\partial}{\partial x_{j}}\right)
$$


Logo

$$
\omega(L)(p)=\sum_{j=1}^{N} L x_{j}(p) \omega\left(\frac{\partial}{\partial x_{j}}\right)(p)=\sum_{j=1}^{N} L_{p}\left(x_{j}\right) \omega\left(\frac{\partial}{\partial x_{j}}\right)(p)=0
$$

Seja $(U, x)$ uma carta local. Como $\left\{\partial_{x_{1}}, \ldots, \partial_{x_{N}}\right\}$ é uma base para $X(U)$ sobre $C^{\infty}(U)$ e como as formas diferenciais são $C^{\infty}$-lineares, uma forma está totalmente determinada em $\mathrm{U}$ pelos seus valores em cada campo $\partial_{x_{j}}$. De importância especial são as formas $d x_{1}, \ldots, d x_{N}$ definidas por

$$
d x_{j}\left(\frac{\partial}{\partial x_{k}}\right)=\delta_{j k} \quad, \quad j, k=1, \ldots, N
$$

isto é, $\left\{d x_{1}, \ldots, d x_{N}\right\}$ é a base dual de $\left\{\partial_{x_{1}}, \ldots, \partial_{x_{N}}\right\}$. Se $\omega \in \Lambda^{1}(U)$ e $L \in X(U)$, temos da representação local para $L$ que $d x_{j}(L)=L x_{j}$; logo

$$
\omega(L)=\sum_{j=1}^{N} L x_{j} \omega\left(\frac{\partial}{\partial x_{j}}\right)=\sum_{j=1}^{N} d x_{j}(L) \omega\left(\frac{\partial}{\partial x_{j}}\right)
$$

e assim

$$
\omega=\sum_{j=1}^{N} \omega\left(\frac{\partial}{\partial x_{j}}\right) d x_{j}
$$

Mais geralmente, se $f \in C^{\infty}(\Omega)$, pode-se definir $d f \in \Lambda^{1}(\Omega)$ por

$$
\mathrm{df}(\mathrm{L})=\mathrm{Lf} \quad, \quad \mathrm{L} \in \mathcal{X}(\Omega)
$$

Para esta forma diferencial, a equação (1.17) toma a forma conhecida

$$
d f=\sum_{j=1}^{N} d f\left(\frac{\partial}{\partial x_{j}}\right) d x_{j}=\sum_{j=1}^{N} \frac{\partial f}{\partial x_{j}} d x_{j}
$$

O espaço cotangente (complexificado) a $\Omega$ em $p$, denotado por $\mathbb{C} T_{p}^{*} \Omega$, é definido como o dual do $\mathbb{C}$-espaço vetorial $\mathbb{C} T_{p} \Omega$, e seus elementos são ditos vetores cotangentes (complexos) a $\Omega$ em p. Portanto um vetor cotangente em p é uma aplicação $\mathbb{C}$-linear

$$
\lambda: \mathbb{C} T_{p} \Omega \rightarrow \mathbb{C}
$$

A cada forma $\omega \in \Lambda^{1}(\Omega)$ está associado um vetor cotangente $\omega_{p}$ de $\mathbb{C T}_{p}^{*} \Omega$, sua localização em $p$, dado por

$$
\omega_{p}(v)=\omega(L)(p) \quad, \quad v \in \mathbb{C T}_{p} \Omega
$$

onde $L \in X(\Omega)$ é qualquer campo tal que $\mathrm{L}_{p}=v$. Este conceito está bem definido em vista das proposições 1.10 e 1.16 . Também está clara a $C^{\infty}(\Omega)$-linearidade da operação de localização, isto é,

$$
\left(\omega_{1}+f \omega_{2}\right)_{p}=\left(\omega_{1}\right)_{p}+f\left(\omega_{2}\right)_{p} \quad, \quad \omega_{1}, \omega_{2} \in \Lambda^{1}(\Omega), f \in C^{\infty}(\Omega)
$$


Observe que, em coordenadas locais, a base dual de $\left\{\left(\partial_{x_{1}}\right)_{p}, \ldots,\left(\partial_{x_{N}}\right)_{p}\right\} \subseteq \mathbb{C T}_{p} \Omega$ é $\left\{\left(d x_{1}\right)_{p}, \ldots,\left(d x_{N}\right)_{p}\right\} \subseteq \mathbb{C T}_{p}^{*} \Omega$. De fato, $\left(d x_{j}\right)_{p}\left(\left(\partial_{x_{k}}\right)_{p}\right)$ é definido como $d x_{j}(L)(p)$ onde L é qualquer campo tal que $L_{p}=\left(\partial_{x_{k}}\right)_{p}$. Podemos então tomar $L=\partial_{x_{k}}$ e obter $\left(d x_{j}\right)_{p}\left(\left(\partial_{x_{k}}\right)_{p}\right)=$ $\mathrm{d} x_{j}\left(\partial_{x_{k}}\right)(p)=\delta_{j k}$, como queríamos. Mais geralmente, se $L \in X(\Omega)$ e $\omega \in \Lambda^{1}(\Omega)$, temos por definição $\omega_{p}\left(L_{p}\right)=\omega(L)(p)$ para todo $p \in \Omega$.

Partindo da representação canônica local de uma forma $\omega \in \Lambda^{1}(\Omega)$,

$$
\omega=\sum_{k=1}^{N} \omega\left(\frac{\partial}{\partial x_{k}}\right) d x_{k}
$$

em torno de um dado $p \in \Omega$, obtemos a fórmula de sua localização em $p$ :

$$
\omega_{p}=\sum_{k=1}^{N} \omega\left(\frac{\partial}{\partial x_{k}}\right)(p)\left(d x_{k}\right)_{p}
$$

Também vale para formas diferenciais um resultado análogo à proposição 1.10 :

Proposição 1.19. $\mathbb{C T}_{\mathrm{p}}^{*} \Omega=\left\{\omega_{\mathrm{p}} ; \omega \in \Lambda^{1}(\Omega)\right\}$ para todo $\mathrm{p} \in \Omega$.

Demonstração: Devido à equação (1.18) e ao fato que $\left\{\left(d x_{k}\right)_{p}\right\}_{k=1, \ldots, N}$ é base para $\mathbb{C T}_{p}^{*} \Omega$, o que precisamos mostrar é que, dada uma carta $U \ni p$ e dados $a_{1}, \ldots, a_{N} \in \mathbb{C}$, existe uma forma $\omega \in \Lambda^{1}(\Omega)$ tal que

$$
\left.\omega\right|_{\mathrm{u}}\left(\frac{\partial}{\partial x_{\mathrm{k}}}\right)(\mathrm{p})=\mathrm{a}_{\mathrm{k}} \quad, \quad \mathrm{k}=1, \ldots, \mathrm{N}
$$

Para tanto, defina $\omega_{0} \in \Lambda^{1}(W)$ em algum aberto $W \subset \subset U$ contendo $p$ como $\omega_{0}=$ $\sum_{k=1}^{N} a_{k} d x_{k}$. Se g é uma função de corte que vale 1 em $W$ e 0 fora de $U$, a forma que procuramos é $\omega=g \omega_{0}$.

Definição 1.20. O fibrado cotangente (complexificado) de $\Omega$ é a reunião disjunta $\mathbb{C T}^{*} \Omega$ dos $\mathbb{C T}_{\mathrm{p}}^{*} \Omega$ sobre todos $\mathrm{p} \in \Omega$. Um subfibrado vetorial (complexo) de $\mathbb{C T}^{*} \Omega$ de posto $\mathbf{m}$ e coposto $\mathbf{N}-\mathbf{m}$ é uma reunião disjunta $\mathcal{W}=\cup_{p \in \Omega} \mathcal{W}_{p}$, onde cada $\mathcal{W}_{\mathrm{p}}$ é um subespaço vetorial de $\mathbb{C T}_{\mathrm{p}}^{*} \Omega$ de dimensão $\mathrm{m}$, satisfazendo a condição de trivialidade local: para todo $p \in \Omega$, existem um aberto $U \ni p$ e formas $\omega_{1}, \ldots, \omega_{m} \in \Lambda^{1}(U)$ de modo que, para cada $\mathrm{q} \in \mathrm{U}, \mathcal{W}_{\mathrm{q}}$ é gerado por $\left(\omega_{1}\right)_{\mathrm{q}}, \ldots,\left(\omega_{\mathrm{m}}\right)_{\mathrm{q}}$. Como antes, diz-se neste caso que $\omega_{1}, \ldots, \omega_{\mathrm{m}}$ geram $\mathcal{W}$ em torno de $\mathbf{p}$. Ainda, $\mathcal{W}_{\mathrm{q}}$ é dita fibra de $\mathcal{W}$ sobre $\mathrm{q}$, e podemos chamar $\mathcal{W}$ de subfibrado cotangente.

Proposição 1.21. Se os campos $\mathrm{L}_{1}, \ldots, \mathrm{L}_{\mathrm{n}} \in X(\Omega)$ são linearmente independentes em uma vizinhança aberta $\mathrm{U}$ de um ponto $\mathrm{p} \in \Omega$, então existem um aberto $\mathrm{V}$, com $\mathrm{p} \in \mathrm{V} \subseteq \mathrm{U}$, e formas $\omega_{1}, \ldots, \omega_{\mathrm{N}-\mathrm{n}} \in \Lambda^{1}(\mathrm{~V})$ linearmente independentes tais que

$$
\omega_{j}\left(L_{k}\right)=0 \text { em } V \quad, \quad j=1, \ldots, N-n, k=1, \ldots, n
$$


Demonstração: Diminua $U$ até que esteja contido em uma carta em torno de $p$, e escreva os campos $\mathrm{L}_{j}$ em U como

$$
L_{j}=\sum_{k=1}^{N} a_{j k} \frac{\partial}{\partial x_{k}} \quad, \quad j=1, \ldots, n
$$

para funções $a_{j k} \in C^{\infty}(U)$. Defina a matriz de funções suaves

$$
\begin{aligned}
A(q) & =\left(\begin{array}{ccc}
a_{11}(q) & \ldots & a_{1 N}(q) \\
\vdots & \ddots & \vdots \\
a_{n 1}(q) & \ldots & a_{n N}(q)
\end{array}\right)_{n \times N}= \\
& =\left(\begin{array}{ccc|ccc}
a_{11}(q) & \ldots & a_{1 n}(q) & a_{1, n+1}(q) & \ldots & a_{1 N}(q) \\
\vdots & \ddots & \vdots & \vdots & \ddots & \vdots \\
a_{n 1}(q) & \ldots & a_{n n}(q) & a_{n, n+1}(q) & \ldots & a_{n N}(q)
\end{array}\right)_{n \times N}= \\
&
\end{aligned}
$$

Então temos

$$
\left(\mathrm{L}_{1}, \ldots, \mathrm{L}_{\mathrm{n}}\right)^{\mathrm{T}}=\mathrm{A}\left(\partial_{\mathrm{x}_{1}}, \ldots, \partial_{\mathrm{x}_{\mathrm{N}}}\right)^{\mathrm{T}}
$$

e como os $L_{j}$ são linearmente independentes, a matriz $A(q)$ tem posto $n$ para todo $q \in U$. Para facilitar a notação, suponha sem perda de generalidade que $C(p)$ é invertível, e tome $\mathrm{V} \subseteq \mathrm{U}$ tal que $\mathrm{C}(\mathrm{q})$ é invertível para todo $\mathrm{q} \in \mathrm{V}$. Defina as matrizes

$$
E(q)=-C^{-1}(q) D(q) \quad, \quad B(q)=\left(\frac{E(q)_{n \times(N-n)}}{I_{N-n}}\right)_{N \times(N-n)}
$$

onde $\mathrm{I}_{\mathrm{N}-\mathrm{n}}$ é a matriz identidade com $\mathrm{N}-\mathrm{n}$ linhas e colunas. Note que $A B=\mathrm{CE}+\mathrm{DI}=$ $-\mathrm{D}+\mathrm{D}=0$. Portanto, basta definirmos formas $\omega_{1}, \ldots, \omega_{N-n} \in \Lambda^{1}(V)$ pela equação

$$
\left(\omega_{1}, \ldots, \omega_{N-n}\right)^{\top}=B\left(d x_{1}, \ldots, d x_{N}\right)^{\top}
$$

Isto é, se $B=\left(b_{j k}\right)$, então $\omega_{i}=\sum_{p=1}^{N} b_{i p} d x_{p}$, e assim

$$
\omega_{i}\left(L_{j}\right)=\sum_{p=1}^{N} \sum_{k=1}^{N} a_{j k} b_{p i} d x_{p}\left(\frac{\partial}{\partial x_{k}}\right)=\sum_{k=1}^{N} a_{j k} b_{k i}
$$

Este é o elemento $(j, i)$ da matriz $A B$, que é zero.

Pode-se obter um subfibrado de $\mathbb{C} T^{*} \Omega$ de posto $N-n$ a partir de um subfibrado $V$ de $\mathbb{C} T \Omega$ de posto $n$, dito complemento ortogonal ou anulador de $\mathcal{V}$, e denotado por $\mathcal{V}^{\perp}$, definindo suas fibras como sendo os subespaços

$$
\nu_{p}^{\perp}=\left\{\lambda \in \mathbb{C T}_{p}^{*} \Omega ; \lambda(v)=0 \quad \forall v \in \nu_{p}\right\} \subseteq \mathbb{C T}_{p}^{*} \Omega
$$


É necessário verificar que se trata de um subfibrado. Que cada fibra é um espaço vetorial $(N-n)$-dimensional está claro; provemos a condição de trivialidade local. Seja $p \in \Omega$; precisamos encontrar um aberto $V \ni p$ e formas $\omega_{1}, \ldots, \omega_{N-n} \in \Lambda^{1}(V)$ tais que, para cada $q \in V$, a fibra $V_{q}^{\perp}$ seja gerada por $\left(\omega_{1}\right)_{q}, \ldots,\left(\omega_{N-n}\right)_{q}$. Mas sabemos, sendo $V$ um subfibrado de $\mathbb{C} T \Omega$, que existem um aberto $U \ni p$ e campos linearmente independentes $\mathrm{L}_{1}, \ldots, \mathrm{L}_{n} \in X(\mathrm{U})$ tais que, para cada $\mathrm{q} \in \mathrm{U}$, a fibra $\mathcal{V}_{\mathrm{q}}$ é gerada por $\left(\mathrm{L}_{1}\right)_{\mathrm{q}}, \ldots,\left(\mathrm{L}_{n}\right)_{\mathrm{q}}$. Então defina $V$ e $\omega_{j}$ tais como dados pela proposição anterior. Fixe $q \in V$. Como as formas $\omega_{j}$ são linearmente independentes, também os vetores cotangentes $\left(\omega_{j}\right)_{q} \circ$ são, para $j=1, \ldots, N-n$. Resta assim mostrar que pertencem a $\mathcal{V}_{q}^{\perp}$; mas de fato, para quaisquer $j=1, \ldots, N-n$ e $k=1, \ldots, n$, temos

$$
\left(\omega_{j}\right)_{q}\left(\left(L_{k}\right)_{q}\right)=\omega_{j}\left(L_{k}\right)(q)=0
$$

logo também $\left(\omega_{\mathrm{j}}\right)_{\mathrm{q}}(v)=0$ para qualquer $v \in \mathcal{V}_{\mathrm{q}}=\operatorname{span}\left\{\left(\mathrm{L}_{1}\right)_{\mathrm{q}}, \ldots,\left(\mathrm{L}_{\mathrm{n}}\right)_{\mathrm{q}}\right\}$, isto é, $\left(\omega_{\mathrm{j}}\right)_{\mathrm{q}} \in$ $V_{\mathrm{q}}^{\perp}$.

\subsection{Campos e formas reais}

Faz-se necessário ao longo do desenvolvimento desta teoria especificar quando campos, vetores, subfibrados etc. são ditos reais. Para tanto, é suficiente estender o conceito de conjugação complexa a essas estruturas. A seguinte definição envolve cálculos trabalhosos porém triviais, necessários para provar que os objetos definidos são de fato campos, formas ou vetores (co-)tangentes:

Definição 1.22. Sejam $L \in X(\Omega), \omega \in \Lambda^{1}(\Omega), v \in \mathbb{C} T_{p} \Omega, \lambda \in \mathbb{C T}_{p}^{*} \Omega$. Definimos seus conjugados $\overline{\mathrm{L}} \in X(\Omega), \bar{\omega} \in \Lambda^{1}(\Omega), \bar{v} \in \mathbb{C T}_{\mathrm{p}} \Omega$ e $\bar{\lambda} \in \mathbb{C T}_{\mathrm{p}}^{*} \Omega$ por

$$
\begin{array}{cl}
\overline{\mathrm{L} f}=\overline{\mathrm{L}(\overline{\mathrm{f}})}, f \in \mathrm{C}^{\infty}(\Omega) & \bar{\omega} \mathrm{L}=\overline{\omega(\overline{\mathrm{L}})}, \mathrm{L} \in \mathcal{X}(\Omega) \\
\bar{v}(\mathrm{f})=\overline{v(\overline{\mathrm{f}})}, f \in \mathrm{C}^{\infty}(\mathrm{p}) & \bar{\lambda}(v)=\overline{\lambda(\bar{v})}, \quad v \in \mathbb{C T}_{p} \Omega
\end{array}
$$

Dizemos que $\mathrm{L}, \omega, v$ e $\lambda$ são reais quando $\mathrm{L}=\overline{\mathrm{L}}, \bar{\omega}=\omega, v=\bar{v}$ e $\lambda=\bar{\lambda}$, respectivamente.

Sejam as seguintes notações:

$$
\begin{gathered}
\mathrm{C}^{\infty}(\Omega ; \mathbb{R})=\left\{\mathrm{f} \in \mathrm{C}^{\infty}(\Omega) ; \mathrm{f} \text { a valores reais }\right\} \\
\mathrm{T}_{\mathrm{p}} \Omega=\left\{v \in \mathbb{C} \mathrm{T}_{\mathrm{p}} \Omega ; v \text { real }\right\} \\
\mathrm{T} \Omega=\bigcup_{\mathrm{p} \in \Omega} \mathrm{T}_{\mathrm{p}} \Omega=\left\{\lambda \in \mathbb{C T}_{\mathrm{p}}^{*} \Omega ; \lambda \text { real }\right\} \\
\mathrm{T}^{*} \Omega=\bigcup_{\mathrm{p} \in \Omega} \mathrm{T}_{\mathrm{p}}^{*} \Omega
\end{gathered}
$$


É simples ver que um campo $L$ é real se e só se $L\left(C^{\infty}(\Omega ; \mathbb{R})\right) \subseteq C^{\infty}(\Omega ; \mathbb{R})$. Ainda, outra condição equivalente é que suas coordenadas num sistema local na base $\left\{\partial_{x_{1}}, \ldots, \partial_{x_{N}}\right\}$ sejam elementos de $C^{\infty}(\Omega ; \mathbb{R})$, e o mesmo vale para formas. A propriedade análoga também vale para vetores (co-)tangentes, dados em coordenadas complexas locais com relação às bases $\left\{\left(\partial_{x_{1}}\right)_{p}, \ldots,\left(\partial_{x_{N}}\right)_{p}\right\}$ ou $\left\{\left(d x_{1}\right)_{p}, \ldots,\left(d x_{N}\right)_{p}\right\}$ : essas coordenadas devem ser números reais.

Seja $\mathcal{V}$ um subfibrado de $\mathbb{C} T \Omega$. Então a reunião disjunta

$$
\bar{\nu}=\bigcup_{p \in \Omega} \overline{\mathcal{V}_{p}}
$$

onde por definição $\overline{V_{p}}=\left\{\bar{v} ; v \in \mathcal{V}_{p}\right\}$, constitui um subfibrado de $\mathbb{C} T \Omega$ de mesmo posto. A justificativa é que, se $L_{1}, \ldots, L_{n}$ geram $V$ em torno de um ponto, então $\overline{L_{1}}, \ldots, \overline{L_{n}}$ geram $\overline{\mathcal{V}}$ em torno do mesmo ponto, e isso se deve ao fato que, para quaisquer $c_{j} \in \mathbb{C}$,

$$
\overline{\sum c_{j}\left(L_{j}\right)_{q}}(f)=\overline{\sum c_{j}\left(L_{j}\right)_{q}(\bar{f})}=\sum \overline{c_{j}} \overline{\left(L_{j}\right)_{q}(\bar{f})}=\sum \overline{c_{j}\left(L_{j}\right)_{q}}(f)
$$

isto é

$$
\overline{\sum \mathrm{c}_{\mathrm{j}}\left(\mathrm{L}_{\mathrm{j}}\right)_{\mathrm{q}}}=\overline{\mathrm{c}_{\mathrm{j}}} \overline{\left(\mathrm{L}_{\mathrm{j}}\right)_{\mathrm{q}}}
$$

A definição análoga vale também caso $\mathcal{V}$ seja subfibrado de $\mathbb{C T}^{*} \Omega$.

Proposição 1.23. Seja $\nu$ subfibrado de $\mathbb{C} T \Omega$. Então

$$
\overline{\mathcal{V}^{\perp}}=\overline{\mathcal{V}}^{\perp}
$$

Demonstração: Basta mostrarmos a igualdade entre as fibras. Fixe $p \in \Omega$. Temos

$$
\begin{aligned}
& \lambda \in{\overline{\mathcal{V}_{p}}}^{\perp} \Longleftrightarrow \lambda(\bar{v})=0 \text { para todo } v \in \mathcal{V}_{p} \\
& \lambda \in \overline{\mathcal{V}_{p}^{\perp}} \Longleftrightarrow \bar{\lambda}(v)=0 \text { para todo } v \in \mathcal{V}_{p}
\end{aligned}
$$

Pela definição de $\bar{\lambda}$, essas duas condições são equivalentes.

\subsection{Teorema de Frobenius}

O Teorema de Frobenius permite encontrar, sob certas condições, coordenadas locais nas quais um subfibrado fica gerado pelos campos da base canônica local de $X(\Omega)$. É constantemente utilizado em trabalhos que buscam resultados de natureza local, uma vez que simplifica as estruturas estudadas sem perda de generalidade. O próximo lema é um caso particular que será utilizado em sua demonstração.

Lema 1.24. Seja $\mathrm{L} \in X(\Omega)$ um campo real que nunca se anula. Então, dado $p \in \Omega$, existem uma vizinhança de $\mathrm{p}$ e um sistema de coordenadas $\mathrm{y}_{1}, \ldots, \mathrm{y}_{\mathrm{N}}$ nesta vizinhança de forma que $\mathrm{L}=\partial_{y_{1}}$. 
Demonstração: Assim como é o caso com qualquer resultado local em variedades, podemos assumir que $\Omega=\mathbb{R}^{N}$ e $p=0$. Sabemos então que existem funções $a_{1}, \ldots, a_{N} \in$ $C^{\infty}(\Omega ; \mathbb{R})$ tais que

$$
L=\sum_{j=1}^{N} a_{j} \frac{\partial}{\partial x_{j}}
$$

Como $L \neq 0$, podem-se reordenar as coordenadas para que tenhamos $a_{1}(0) \neq 0$. Considere então o seguinte problema de Cauchy para um sistema de EDO's dadas em funções $x_{1}, \ldots, x_{N}$ das variáveis $y_{1}, \ldots, y_{N}$ :

$$
\left\{\begin{array}{l}
\frac{\partial x_{j}}{\partial y_{1}}=a_{j}\left(x_{1}, \ldots, x_{N}\right), \quad j=1, \ldots, N \\
x_{1}\left(0, y_{2}, \ldots, y_{N}\right)=0 \\
x_{j}\left(0, y_{2}, \ldots, y_{N}\right)=y_{j}, j=2, \ldots, N
\end{array}\right.
$$

Este sistema tem solução única $x_{1}, \ldots, \chi_{N}$ em torno de 0 , e é crucial para tanto a hipótese que as $a_{j}$ são funções reais. O determinante Jacobiano da aplicação $\left(y_{1}, \ldots, y_{N}\right) \mapsto$ $\left(x_{1}, \ldots, x_{N}\right)$ é

$$
\left|\begin{array}{cccc}
a_{1} & 0 & \ldots & 0 \\
a_{2} & 1 & \ldots & 0 \\
\vdots & \vdots & \ddots & \vdots \\
a_{N} & 0 & \ldots & 1
\end{array}\right|=a_{1}\left(y_{1}, \ldots, y_{N}\right)
$$

Como $a_{1}$ é não-nulo em 0 , temos pelo Teorema da Função Inversa que a aplicação acima é uma mudança de coordenadas (isto é, um difeomorfismo suave) numa vizinhança de 0 . Agora observe que

$$
\begin{aligned}
& 0=\frac{\partial y_{j}}{\partial y_{1}}=\sum_{k=1}^{N} \frac{\partial y_{j}}{\partial x_{k}} \frac{\partial x_{k}}{\partial y_{1}}=\sum_{k=1}^{N} a_{k} \frac{\partial y_{j}}{\partial x_{k}}=L y_{j}, j=2, \ldots, N \\
& 1=\frac{\partial y_{1}}{\partial y_{1}}=\sum_{k=1}^{N} \frac{\partial y_{1}}{\partial x_{k}} \frac{\partial x_{k}}{\partial y_{1}}=\sum_{k=1}^{N} a_{k} \frac{\partial y_{1}}{\partial x_{k}}=L y_{1}
\end{aligned}
$$

Portanto, L escrito nas coordenadas y tem a forma

$$
L=\sum_{j=1}^{N}\left(L y_{j}\right) \frac{\partial}{\partial y_{j}}=\frac{\partial}{\partial y_{1}}
$$

Teorema 1.25. (Frobenius) Seja $\mathcal{V}$ um subfibrado de $\mathbb{C} T \Omega$. Sejam $\mathrm{L}_{1}, \ldots, \mathrm{L}_{n}$ campos reais linearmente independentes que geram $\mathcal{V}$ em torno de $p \in \Omega$. Se $\mathcal{V}$ é uma estrutura involutiva, então existem coordenadas $\mathrm{y}_{1}, \ldots, \mathrm{y}_{\mathrm{N}}$ em torno de $\mathrm{p}$ tais que $\mathcal{V}$ é gerada em torno de $p$ pelos campos $\partial_{y_{1}}, \ldots, \partial_{y_{n}}$. 
Demonstração: Assuma como antes $\Omega=\mathbb{R}^{N}$ e $p=0$. Prova-se por indução em $N$. Se $N=1$, também temos $n=1$, e reduzimos o problema ao lema acima. Agora suponha que o resultado vale para $N-1$ no lugar de N. Escreva os campos $L_{j}$ em torno de 0 na forma

$$
\mathrm{L}_{j}=\sum_{\mathrm{k}=1}^{\mathrm{N}} \mathrm{a}_{\mathrm{jk}} \frac{\partial}{\partial \mathrm{x}_{\mathrm{k}}}
$$

Como o que queremos provar é um resultado local, a fórmula para os $L_{j}$ longe de 0 não importa; logo podemos assumir que a representação acima é válida em $\mathbb{R}^{\mathrm{N}}$ (estendendo-se as $a_{j k}$ através de funções de corte). Aplique o lema ao campo $L_{1}$ para que tenhamos, sem perda de generalidade, $a_{11} \equiv 1$ e $a_{1 k} \equiv 0(k \geq 2)$, e sejam

$$
\begin{aligned}
& \mathrm{L}_{1}^{\sharp}=\mathrm{L}_{1} \\
& \mathrm{~L}_{\mathrm{j}}^{\sharp}=\mathrm{L}_{\mathrm{j}}-\mathrm{a}_{\mathrm{j} 1} \mathrm{~L}_{1} \quad, \quad \mathrm{j} \geq 2
\end{aligned}
$$

Os campos $L_{j}^{\sharp}$ são linearmente independentes, portanto continuam gerando $\mathcal{V}$, e o teorema 1.13 garante que existem $\lambda_{j l}^{k} \in C^{\infty}\left(\mathbb{R}^{N}\right)$ tais que

$$
\left[\mathrm{L}_{j}^{\sharp}, \mathrm{L}_{\mathrm{l}}^{\sharp}\right]=\sum_{\mathrm{k}=1}^{\mathrm{n}} \lambda_{j l}^{\mathrm{k}} \mathrm{L}_{\mathrm{k}}^{\sharp}
$$

Um cálculo direto mostra que $\lambda_{j l}^{1}=0$ para $j, l \geq 2$. De fato, a representação local do colchete de Lie é dada por

$$
\left[L_{j}^{\sharp}, L_{l}^{\sharp}\right]=\sum_{k=1}^{N}\left[L_{j}^{\sharp} L_{l}^{\sharp} x_{k}-L_{l}^{\sharp} L_{j}^{\sharp} x_{k}\right] \frac{\partial}{\partial x_{k}}
$$

e, para $j \geq 2$, os campos $L_{j}^{\sharp}$ não têm a derivada $\partial_{x_{1}}$, logo $L_{j}^{\sharp} x_{k}=0, k=2, \ldots, N$.

Sejam

$$
M_{j}=\sum_{k=2}^{N} a_{j k}\left(0, x_{2}, \ldots, x_{N}\right) \frac{\partial}{\partial x_{k}} \quad, \quad j=2, \ldots, n
$$

Provemos que os campos $M_{j} \in X\left(\mathbb{R}^{N-1}\right)$ são linearmente independentes. Suponha que existam funções $\alpha_{j} \in C^{\infty}\left(\mathbb{R}^{N-1}\right)$ tais que

$$
\sum_{j=2}^{n} \alpha_{j} M_{j}=0 \text { em } \mathbb{R}^{N-1}
$$

Queremos provar que as $\alpha_{j}$ são funções nulas. Dado $q=\left(q_{2}, \ldots, q_{N}\right) \in \mathbb{R}^{N-1}$, definamos $\tilde{q}=\left(0, q_{2}, \ldots, q_{N}\right) \in \mathbb{R}^{N}$, e dada $f \in C^{\infty}\left(\mathbb{R}^{N}\right)$, definamos $\hat{f} \in C^{\infty}\left(\mathbb{R}^{N-1}\right)$ por $\hat{f}\left(x_{2}, \ldots, x_{N}\right)=$ $f\left(0, x_{2}, \ldots, x_{N}\right)$. Observe inicialmente que, para toda $f \in C^{\infty}\left(\mathbb{R}^{N}\right)$, todo $q=\left(q_{2}, \ldots, q_{N}\right) \in$ 
$\mathbb{R}^{\mathrm{N}-1}$ e todo $j=2, \ldots, N$

$$
\begin{aligned}
L_{j}^{\sharp} f(\tilde{q}) & =\sum_{k=2}^{N} a_{j k}\left(0, q_{2}, \ldots, q_{N}\right) \frac{\partial f}{\partial x_{k}}(\tilde{q}) \\
& =\sum_{k=2}^{N} a_{j k}\left(0, q_{2}, \ldots, q_{N}\right) \frac{\partial \hat{f}}{\partial x_{k}}(q)=M_{j} \hat{f}(q)
\end{aligned}
$$

Dessa forma, temos

$$
\sum_{j=2}^{n} \alpha_{j}(q)\left(L_{j}^{\sharp}\right)_{\tilde{q}}(f)=\sum_{j=2}^{n} \alpha_{j}(q)\left(L_{j}^{\sharp} f\right)(\tilde{q})=\sum_{j=2}^{n} \alpha_{j}(q) M_{j} \hat{f}(q)=\left(\sum_{j=2}^{n} \alpha_{j} M_{j} \hat{f}\right)(q)=0
$$

Segue da arbitrariedade de $f$ que $\sum_{j=2}^{n} \alpha_{j}(q)\left(L_{j}^{\sharp}\right)_{\tilde{q}}=0$. Mas como os vetores tangentes $\left(L_{j}^{\sharp}\right)_{\tilde{q}}$ são linearmente independentes, obtemos $\alpha_{j}(q)=0$ para todo $q$, como queríamos.

Note que os campos $M_{j}, j=2, \ldots, n$, também geram uma estrutura involutiva $V^{\prime}$ sobre $\mathbb{R}^{N-1}$. Para tanto, basta vermos que $\left[M_{j}, M_{l}\right]$ é uma seção, isto é, $\left[M_{j}, M_{l}\right]_{q}$ é dado como combinação $\mathbb{C}$-linear dos $\left(M_{k}\right)_{q}$ para cada $q \in \mathbb{R}^{N-1}$. E de fato, de (1.26) e (1.27), obtemos

$$
\left[M_{j}, M_{l}\right]=\sum_{k=2}^{n} \widehat{\lambda_{j l}^{k}} M_{k}
$$

Logo, para qualquer $q \in \mathbb{R}^{N-1}$, tem-se $\left[M_{j}, M_{l}\right]_{q}=\sum_{k=2}^{n} \widehat{\lambda_{l j}^{k}}(q) M_{k_{q}} \in \mathcal{V}_{q}^{\prime}$.

Dessa forma, podemos aplicar a hipótese de indução a $\mathcal{V}^{\prime}$ para obter coordenadas $y_{2}, \ldots, y_{N}$ de $\mathbb{R}^{N-1}$ tais que

$$
\frac{\partial}{\partial y_{2}}, \ldots, \frac{\partial}{\partial y_{n}}
$$

gerem $V^{\prime}$ em torno de 0 . Se definirmos $y_{1}=x_{1}$, a aplicação $\left(x_{1}, \ldots, x_{N}\right) \mapsto\left(y_{1}, \ldots, y_{N}\right)$ é uma mudança de coordenadas, e temos

$$
\begin{aligned}
& \mathrm{L}_{1}^{\sharp}=\frac{\partial}{\partial x_{1}}=\frac{\partial}{\partial y_{1}} \\
& L_{j}^{\sharp}=\sum_{k=1}^{N} \tilde{a}_{j k} \frac{\partial}{\partial y_{k}} \quad, \quad j=2, \ldots, n
\end{aligned}
$$

para certas funções $\tilde{a}_{j k} ;$ portanto $\nu$ está gerado pelos campos $\frac{\partial}{\partial y_{1}}, \ldots, \frac{\partial}{\partial y_{n}}, \ldots, \frac{\partial}{\partial y_{N}}$.

$\mathrm{O}$ objetivo agora é mostrar que $\tilde{a}_{j k}=0$ para $j=2, \ldots, n$ e $k=n+1, \ldots, N$. Dessa forma $\mathcal{V}$ ficará gerado apenas por

$$
\frac{\partial}{\partial y_{1}}, \ldots, \frac{\partial}{\partial y_{n}}
$$


o que era a tese. É simples ver que, para $k>n, \tilde{a}_{j k} \equiv 0$ em $\left\{x_{1}=0\right\}$, tendo em conta que $\mathcal{V}^{\prime}$ é gerado apenas por $\partial_{y_{2}}, \ldots, \partial_{y_{n}}$. Agora, por um lado,

$$
\left[L_{1}^{\sharp}, L_{j}^{\sharp}\right]=\sum_{k=1}^{n} C_{j l}^{k} L_{k}^{\sharp}=C_{1 j}^{1} \frac{\partial}{\partial y_{1}}+\sum_{k=2}^{n} C_{1 j}^{k} \sum_{l=2}^{N} \tilde{a}_{k l} \frac{\partial}{\partial y_{l}}
$$

e por outro,

$$
\begin{aligned}
& {\left[L_{1}^{\sharp}, L_{j}^{\sharp}\right] f=L_{1}^{\sharp}\left(L_{j}^{\sharp} f\right)-L_{j}^{\sharp}\left(L_{1}^{\sharp} f\right)=\frac{\partial}{\partial y_{1}}\left(\sum_{l=2}^{N} \tilde{a}_{j l} \frac{\partial f}{\partial y_{l}}\right)-\left(\sum_{l=2}^{N} \tilde{a}_{j l} \frac{\partial}{\partial y_{l}} \frac{\partial f}{\partial y_{1}}\right)=} \\
&=\sum_{l=2}^{N} \frac{\partial \tilde{a}_{j l}}{\partial y_{1}} \frac{\partial f}{\partial y_{l}} \quad, \quad f \in C^{\infty}\left(\mathbb{R}^{N}\right)
\end{aligned}
$$

isto é,

$$
\left[\mathrm{L}_{1}^{\sharp}, \mathrm{L}_{\mathrm{j}}^{\sharp}\right]=\sum_{\mathrm{l}=2}^{\mathrm{N}} \frac{\partial \tilde{\mathrm{a}}_{\mathrm{jl}}}{\partial \mathrm{y}_{1}} \frac{\partial}{\partial \mathrm{y}_{\mathrm{l}}}
$$

Igualando-se os coeficientes de $\partial_{y_{\imath}}$ entre as expressões (1.28) e (1.29), obtém-se:

$$
\frac{\partial \tilde{a}_{j l}}{\partial y_{1}}=\sum_{k=2}^{n} C_{1 j}^{k} \tilde{a}_{k l}
$$

Portanto, se $k>n$, o vetor $\left(\tilde{a}_{2 k}, \ldots, \tilde{a}_{n k}\right)$ satisfaz o seguinte problema de Cauchy:

$$
\left\{\begin{array}{l}
\left.\left(\tilde{\mathrm{a}}_{2 \mathrm{k}}, \ldots, \tilde{\mathrm{a}}_{\mathrm{nk}}\right)\right|_{\left\{\mathrm{x}_{1}=0\right\}}=0 \\
\frac{\partial}{\partial \mathrm{y}_{1}}\left(\begin{array}{c}
\tilde{\mathrm{a}}_{2 \mathrm{k}} \\
\vdots \\
\tilde{\mathrm{a}}_{\mathrm{nk}}
\end{array}\right)=\left(C_{1 \mathrm{j}}^{\mathrm{l}}\right)_{(\mathrm{n}-1) \times(\mathrm{n}-1)}\left(\begin{array}{c}
\tilde{\mathrm{a}}_{2 \mathrm{k}} \\
\vdots \\
\tilde{\mathrm{a}}_{\mathrm{nk}}
\end{array}\right)
\end{array}\right.
$$

Por unicidade local da solução de sistemas de EDO's, tal como no lema 1.24, obtemos $\left(\tilde{\mathrm{a}}_{2 \mathrm{k}}, \ldots, \tilde{\mathrm{a}}_{\mathrm{nk}}\right) \equiv 0$ para $\mathrm{k}>\mathrm{n}$.

\subsection{Estruturas holomorfas}

Denote por $\mathbb{C}^{N}$ o conjunto $\left\{x+i y ; x, y \in \mathbb{R}^{N}\right\}$, que identificamos com $\mathbb{R}^{2 N}$, e seja $\Sigma \subseteq \mathbb{C}^{\mathrm{N}}$ um aberto. $\Sigma$ adquire dessa forma uma estrutura de variedade $2 \mathrm{~N}$-dimensional, mas, definindo-se as variáveis complexas $z_{j}=x_{j}+i y_{j}$, podemos também considerar a álgebra $\mathcal{O}(\Sigma)$ das funções holomorfas em $\Sigma$ (isto é, holomorfas em cada $z_{\mathrm{j}}$ ), e assim $\Sigma$ pode ser vista como uma variedade holomorfa. Um sistema de coordenadas holomorfas em um aberto $U \subseteq \Sigma$ é um conjunto $\left\{\mathcal{w}_{1}, \ldots, w_{N}\right\}$ de funções holomorfas em $U$ tais que a aplicação

$$
\left(z_{1}, \ldots, z_{N}\right) \mapsto\left(w_{1}, \ldots, w_{N}\right)
$$

seja um biholomorfismo de $\mathrm{U}$. 
Definição 1.30. Um campo $\mathrm{L} \in \mathcal{X}(\Sigma)$ é dito ser holomorfo se

$$
\text { Lf } \in \mathcal{O}(\Sigma) \quad \text { e } \quad \overline{\mathrm{f}}=0 \quad \text { para toda } \quad \mathrm{f} \in \mathcal{O}(\Sigma)
$$

Todo campo $L \in X(\Sigma)$ se escreve como combinação linear sobre $C^{\infty}(\Sigma)$ dos campos

$$
\begin{array}{cc}
\frac{\partial}{\partial z_{j}}=\frac{1}{2}\left(\frac{\partial}{\partial x_{j}}-i \frac{\partial}{\partial y_{j}}\right), \quad j=1, \ldots, N \\
\frac{\partial}{\partial \overline{z_{j}}}=\frac{1}{2}\left(\frac{\partial}{\partial x_{j}}+i \frac{\partial}{\partial y_{j}}\right), \quad j=1, \ldots, N
\end{array}
$$

e vê-se que L será holomorfo se e somente se puder ser escrito apenas como combinação $\mathcal{O}(\Sigma)$-linear dos campos

$$
\frac{\partial}{\partial z_{1}}, \ldots, \frac{\partial}{\partial z_{N}}
$$

Isto segue do fato que $\left(\partial / \partial \overline{z_{j}}\right) f=0, j=1, \ldots, N$, se $f$ é holomorfa, o que por sua vez é consequência das Equações de Cauchy-Riemann.

O teorema de Frobenius vale também para campos holomorfos sobre uma variedade como $\Sigma$ acima, e a mesma demonstração dada no primeiro caso vale neste também, porque o teorema de existência e unicidade para soluções de um sistema de EDO's é válido também no caso holomorfo:

Teorema 1.31. (Frobenius - Versão Holomorfa) Seja $\mathcal{V}$ um subfibrado de $\mathbb{C} T \Sigma$, onde $\Sigma \subseteq \mathbb{C}^{\mathrm{N}}$ é um aberto. Sejam $\mathrm{L}_{1}, \ldots, \mathrm{L}_{\mathrm{n}}$ campos holomorfos linearmente independentes que geram $\mathcal{V}$ em torno de $p \in \Sigma$. Se $\mathcal{V}$ é involutiva, então existem coordenadas holomorfas $z_{1}, \ldots, z_{N}$ em torno de $p$ tais que $V$ é gerado em torno de $p$ pelos campos $\partial_{z_{1}}, \ldots, \partial_{z_{n}}$.

\subsection{Conjunto característico}

Definição 1.32. O símbolo de um campo $\mathrm{L} \in \mathcal{X}(\Omega)$ é a função $\sigma(\mathrm{L}): \mathrm{T}^{*} \Omega \rightarrow \mathbb{C}$ definida por

$$
\sigma(\mathrm{L})(\lambda)=\lambda\left(\mathrm{L}_{\mathrm{p}}\right) \quad, \quad \lambda \in \mathrm{T}_{\mathrm{p}}^{*} \Omega
$$

para cada $p \in \Omega$.

Suponha que L esteja dado em coordenadas locais:

$$
L=\sum_{j=1}^{N} a_{j} \frac{\partial}{\partial x_{j}}
$$

Seja $\lambda \in \mathrm{T}_{\mathrm{p}}^{*} \Omega$. Sejam $\lambda_{j}, j=1, \ldots, N$, as coordenadas (reais) de $\lambda$ na base canônica de $\mathbb{C T}_{\mathrm{p}}^{*} \Omega$, isto é,

$$
\lambda=\sum \lambda_{j}\left(d x_{j}\right)_{p}
$$


Sabemos pela proposição 1.19 que $\lambda=\omega_{p}$ para alguma $\omega \in \Lambda^{1}(\Omega)$. Note inicialmente que, como $\omega=\sum \omega\left(\partial_{x_{j}}\right) d x_{j}$ e $\lambda=\omega_{p}$, vale a igualdade

$$
\omega\left(\frac{\partial}{\partial x_{j}}\right)(p)=\lambda_{j}
$$

Logo,

$$
\begin{aligned}
& \sigma(L)(\lambda)=\lambda\left(L_{p}\right)=\lambda\left(\sum\right.\left.a_{j}(p)\left(\frac{\partial}{\partial x_{j}}\right)_{p}\right)=\sum a_{j}(p) \lambda\left(\frac{\partial}{\partial x_{j}}\right)_{p}= \\
&=\sum a_{j}(p) \omega_{p}\left(\frac{\partial}{\partial x_{j}}\right)_{p}=\sum a_{j}(p) \omega\left(\frac{\partial}{\partial x_{j}}\right)(p)=\sum a_{j}(p) \lambda_{j}
\end{aligned}
$$

Isto é, se L é um operador diferencial parcial linear homogêneo de primeira ordem em $\mathbb{R}^{\mathrm{N}}$, a definição de símbolo coincide com a de seu polinômio símbolo, desde que identifiquemos $\mathrm{T}_{\mathrm{p}}^{*}\left(\mathbb{R}^{\mathrm{N}}\right) \operatorname{com} \mathbb{R}^{\mathrm{N}}$.

Definição 1.33. O conjunto característico de uma estrutura involutiva $v$ é definido por

$$
\mathrm{T}^{0}=\mathcal{V}^{\perp} \cap \mathrm{T}^{*} \Omega
$$

isto é, são os vetores cotangentes reais que estão no anulador de $\mathcal{V}$. Também se definem as fibras

$$
\mathrm{T}_{\mathrm{p}}^{0}=\mathcal{V}_{\mathrm{p}}^{\perp} \cap \mathrm{T}_{\mathrm{p}}^{*} \Omega
$$

para cada $\mathrm{p} \in \Omega$ (muito embora $\mathrm{T}^{0}$ não seja em geral um subfibrado vetorial sobre $\mathbb{R})$.

Proposição 1.34. Fixe um subfibrado tangente $\mathcal{V}$ e seja $\lambda \in \mathrm{T}_{\mathrm{p}}^{*} \Omega$. Então $\lambda \in \mathrm{T}_{\mathrm{p}}^{0}$ se e somente se $\sigma(\mathrm{L})(\lambda)=0$ para todo $\mathrm{L}$ seção de $\mathcal{V}$ sobre alguma vizinhança aberta de $p$.

Demonstração: Se L é seção em torno de $p$, então $\mathrm{L}_{p} \in \mathcal{V}_{\mathrm{p}}$, e de $\lambda \in \mathrm{T}_{\mathrm{p}}^{0} \subseteq \mathcal{V}_{\mathrm{p}}^{\perp}$ obtemos $\sigma(L)(\lambda)=\lambda\left(L_{p}\right)=0$. Reciprocamente, suponha que $\sigma(L)(\lambda)=0$ para toda seção $L$ em torno de $p$; resta provar que $\lambda \in \mathcal{V}_{p}^{\perp}$. Existem campos $L_{1}, \ldots, L_{n}$ que geram $\mathcal{V}$ em torno de $p$, e assim basta verificar que $\lambda\left(\left(L_{j}\right)_{p}\right)=0$ para $j=1, \ldots, n$. Mas isto segue da hipótese que $\sigma(L)(\lambda)=0$ para toda seção L em torno de $p$, e do fato que, por gerarem $\mathcal{V}$, os campos $L_{j}$ são seções em torno de $p$.

Essa proposição implica que, se $\mathcal{V}$ é gerado localmente por

$$
L_{j}=\sum_{k=1}^{N} a_{j k} \frac{\partial}{\partial x_{k}} \quad, \quad j=1, \ldots, n
$$

então a condição $\lambda \in \mathrm{T}_{\mathrm{p}}^{0}$ é caracterizada por

$$
\lambda_{j} \in \mathbb{R}, \quad j=1, \ldots, n
$$




$$
\sum_{k=1}^{N} a_{j k}(p) \lambda_{k}=0, j=1, \ldots, n
$$

o que constitui a definição de conjunto característico no ponto $p$ de um conjunto de operadores diferenciais parciais lineares homogêneos de primeira ordem $L_{1}, \ldots, L_{n}$ em $\mathbb{R}^{N}$ dados como acima.

Para cada $p$, o conjunto $\mathrm{T}_{\mathrm{p}}^{0}$ é um $\mathbb{R}$-espaço vetorial. Uma condição necessária para que $\mathrm{T}^{0}$ constituísse um subfibrado vetorial real seria que todas as fibras tivessem a mesma dimensão real. O exemplo a seguir mostra que em geral essa propriedade não é verdadeira.

Exemplo 1.35. (Estrutura de Mizohata) O operador

$$
M=\partial_{t}-i t \partial_{x}
$$

definido em $\mathbb{R}^{2}=\{(x, t) ; x, t \in \mathbb{R}\}$, é chamado operador de Mizohata. Analisemos a estrutura involutiva gerada em $\mathbb{R}^{2}$ por $M$, visto como um campo vetorial complexo.

Se $p=\left(p_{1}, p_{2}\right) \in \mathbb{R}^{2}$, temos $M_{p}=-i p_{2}\left(\partial_{x}\right)_{p}+\left(\partial_{t}\right)_{p}$, que identificamos com $\left(-\mathrm{ip}_{2}, 1\right) \in \mathbb{C}^{2}$, tendo-se em conta que $\mathbb{C T}_{\mathrm{p}} \mathbb{R}^{2} \approx \mathbb{C}^{2}$ e que a base canônica de $\mathbb{C T}_{\mathrm{p}} \mathbb{R}^{2}$ sobre $\mathbb{C}$ é $\left\{\left(\partial_{x}\right)_{p},\left(\partial_{t}\right)_{p}\right\}$. Observe que $M_{p} \neq 0$ para qualquer $p \in \mathbb{R}^{2}$, logo $M$ gera um subfibrado tangente de posto 1 sobre $\mathbb{R}^{2}$.

Seja $\lambda=\left(\lambda_{1}, \lambda_{2}\right) \in \mathbb{R}^{2} \approx \mathrm{T}_{\mathrm{p}}^{*} \mathbb{R}^{2}$. Temos

$$
\sigma(M)(\lambda)=\lambda\left(M_{p}\right)=-i p_{2} \lambda_{1}+\lambda_{2}
$$

Logo, se $\mathrm{p}_{2}=0$, o conjunto

$$
\mathrm{T}_{\mathrm{p}}^{0}=\left\{\lambda \in \mathbb{R}^{2} ; \sigma(M)(\lambda)=0\right\}=\left\{\lambda \in \mathbb{R}^{2} ; \lambda_{2}=0\right\}
$$

é um $\mathbb{R}$-espaço vetorial de dimensão 1 . Por outro lado, se $p_{2} \neq 0$, tem-se que

$$
\mathrm{T}_{\mathrm{p}}^{0}=\left\{\lambda \in \mathbb{R}^{2} ; \sigma(\mathrm{M})(\lambda)=0\right\}=\{(0,0)\}
$$

é um $\mathbb{R}$-espaço vetorial de dimensão 0. Dessa forma, $\mathrm{T}^{0}$ não pode ser um subfibrado sobre $\mathbb{R}^{2}$.

\subsection{Tipos de estruturas}

Seja $\mathcal{V}$ um subfibrado tangente. Dizemos que $\mathcal{V}$ é uma estrutura:

- elíptica se para todo $p \in \Omega$ vale $T_{p}^{0}=0$;

- de Cauchy-Riemann (CR) se para todo $p \in \Omega$ vale $\mathcal{V}_{p}^{\perp}+\overline{\mathcal{V}_{p}^{\perp}}=\mathbb{C T}_{p}^{*} \Omega$; 
- complexa se para todo $p \in \Omega$ vale $\mathcal{V}_{\mathrm{p}}^{\perp} \oplus \overline{\mathcal{V}_{\mathrm{p}}^{\perp}}=\mathbb{C T}_{\mathrm{p}}^{*} \Omega$;

- essencialmente real se para todo $p \in \Omega$ vale $\mathcal{V}_{p}^{\perp}=\overline{\mathcal{V}_{p}^{\perp}}$.

Obviamente, também se pode dizer que uma estrutura é de um certo tipo apenas no ponto p. Uma estrutura essencialmente real no ponto $p$ é comumente chamada uma estrutura generalizada de Mizohata no ponto $\mathbf{p}$, tendo-se em vista o que ocorre com o operador de Mizohata.

Aplicando-se propriedades elementares de Álgebra Linear a respeito de espaços anuladores, é possível obter caracterizações para os três últimos casos em termos de $\mathcal{V}$ ao invés de $\mathcal{V}^{\perp}$. Elas são:

- $V$ é $C R$ se e somente se para todo $p \in \Omega$ vale $\mathcal{V}_{p} \cap \overline{\mathcal{V}_{p}}=0$;

- $\mathcal{V}$ é complexa se e somente se para todo $p \in \Omega$ vale $\mathcal{V}_{p} \oplus \overline{\mathcal{V}_{p}}=\mathbb{C} T_{p} \Omega$;

- $V$ é essencialmente real se e somente se para todo $p \in \Omega$ vale $\mathcal{V}_{p}=\overline{\mathcal{V}_{p}}$.

Mas obter uma caracterização similar para o caso elíptico requer alguns cálculos adicionais:

Lema 1.36. Seja $\vee$ um $\mathbb{C}$-subespaço vetorial de $\mathbb{C}^{N}=\mathbb{R}^{N}+i \mathbb{R}^{N}$ e considere o $\mathbb{R}$-espaço vetorial

$$
\mathrm{V}^{0}=\mathrm{V} \cap \mathbb{R}^{\mathrm{N}}=\{z \in \mathrm{V} ; z=\bar{z}\}
$$

Então $\mathrm{V} \cap \overline{\mathrm{V}}=\mathrm{V}^{0}+i \mathrm{~V}^{0}$

Demonstração: Seja $z \in V \cap \bar{V}$. Então $z, \bar{z} \in V$, o que implica que $(1 / 2)(z+\bar{z})$ e $(1 / 2)(i \bar{z}-i z)$ pertencem a $V^{0}$. Em particular, $z=(1 / 2)(z+\bar{z})+i(1 / 2)(i \bar{z}-i z) \in V^{0}+i V^{0}$. Reciprocamente, suponha que $z \in \mathrm{V}^{0}+i \mathrm{~V}^{0}$; então $z=x+i y \operatorname{com} x, y \in \mathrm{V}^{0}$, e segue que $z \in \mathrm{V}$ e $z=\overline{x-i y} \in \bar{V}$, isto é, $z \in \mathrm{V} \cap \overline{\mathrm{V}}$.

Corolário 1.37. Um subfibrado tangente $\mathcal{V}$ é uma estrutura elíptica se e somente se para cada $\mathrm{p} \in \Omega$ vale

$$
\nu_{p}+\overline{\nu_{p}}=\mathbb{C} T_{p} \Omega
$$

Demonstração: Seja $p \in \Omega$. Através da identificação $\mathcal{V}_{p} \approx \mathbb{C}^{n}$, e portanto também $\mathrm{T}_{\mathrm{p}}^{0} \approx \mathbb{R}^{n}$, o lema acima se aplica com $\mathcal{V}_{\mathrm{p}}^{\perp}$ no lugar de $\mathrm{V}$ e $\mathrm{T}_{\mathrm{p}}^{0}$ no lugar de $\mathrm{V}^{0}$, fornecendo

$$
T_{p}^{0}+i T_{p}^{0}=\mathcal{V}_{p}^{\perp} \cap \overline{\mathcal{V}_{p}^{\perp}}
$$

Logo a condição $T_{p}^{0}=0$, equivalente a $T_{p}^{0}+i T_{p}^{0}=0$, é caracterizada pela condição $\mathcal{V}_{p}^{\perp} \cap \overline{\mathcal{V}_{p}^{\perp}}=$ 0 , ou ainda, tomando os anuladores em ambos os lados, $\nu_{p}+\overline{\nu_{p}}=\mathbb{C T}_{p} \Omega$.

Tendo em mãos as caracterizações deduzidas, vê-se que um subfibrado tangente é uma estrutura complexa se e somente se é CR e elíptica. 
Proposição 1.38. Um subfibrado de $\mathbb{C} T \Omega$ é essencialmente real se e somente se é localmente gerado por campos reais. O mesmo vale para subfibrados de $\mathbb{C T}^{*} \Omega$, com formas reais ao invés de campos reais.

Demonstração: Mostraremos a proposição apenas para subfibrados tangentes, pois a situação no fibrado cotangente é inteiramente análoga. Suponha inicialmente que n campos reais $L_{1}, \ldots, L_{n}$ gerem um subfibrado $\mathcal{V}$ em um aberto $U$ em torno de $p \in \Omega$, e seja $v \in \overline{\mathcal{V}_{p}}$. Observe que

$$
\overline{\left(\mathrm{L}_{\mathrm{j}}\right)_{\mathrm{p}}}=\left(\mathrm{L}_{\mathrm{j}}\right)_{\mathrm{p}}, \quad \mathrm{j}=1, \ldots, \mathrm{n}
$$

pela definição de $\left(L_{j}\right)_{p}$ e por $L_{j}$ ser real. Além disso, existem escalares $a_{1}, \ldots, a_{n}$ tais que

$$
\bar{v}=\sum_{j=1}^{n} a_{j}\left(L_{j}\right)_{p} \quad \text { em } u
$$

Temos assim

$$
v=\sum_{j=1}^{n} \overline{a_{j}} \overline{\left(L_{j}\right)_{p}}=\sum_{j=1}^{n} \overline{a_{j}}\left(L_{j}\right)_{p} \in \mathcal{V}_{p}
$$

o que prova que $\overline{\nu_{p}} \subseteq \mathcal{V}_{p}$. Analogamente, $\mathcal{V}_{\mathrm{p}} \subseteq \overline{\mathcal{V}_{\mathrm{p}}}$, logo $\mathcal{V}$ é essencialmente real.

Reciprocamente, sejam $n$ campos $L_{1}, \ldots, L_{n} \in X(\Omega)$ que geram um subfibrado $V$ essencialmente real em torno de algum $p$. Defina os campos reais

$$
\mathfrak{R} \mathrm{L}_{j}=(1 / 2)\left(\mathrm{L}_{\mathrm{j}}+\overline{\mathrm{L}_{j}}\right) \quad, \quad \mathfrak{I} \mathrm{L}_{\mathrm{j}}=(1 / 2 i)\left(\mathrm{L}_{\mathrm{j}}-\overline{\mathrm{L}_{\mathrm{j}}}\right) \quad, \quad \mathrm{j}=1, \ldots, \mathfrak{n}
$$

Observe que

$$
\mathrm{L}_{j}=\mathfrak{R} \mathrm{L}_{j}+i \mathfrak{I} \mathrm{L}_{j} \quad, \quad j=1, \ldots, n
$$

Da hipótese que $\mathcal{V}_{\mathrm{q}}=\overline{\mathcal{V}_{\mathrm{q}}}$ para todo $\mathrm{q} \in \Omega$, segue que os campos $\overline{\mathrm{L}_{\mathrm{j}}}$, e portanto também os campos $\mathfrak{R} L_{j}$ e $\mathfrak{I} L_{j}$, são seções de $\mathcal{V}$. Em particular,

$$
\left(\mathfrak{R} \mathrm{L}_{\mathrm{j}}\right)_{\mathrm{p}},\left(\mathfrak{I} \mathrm{L}_{\mathrm{j}}\right)_{\mathrm{p}} \in \mathcal{V}_{\mathrm{p}} \quad, \quad \mathfrak{j}=1, \ldots, \mathrm{n}
$$

e portanto, da definição de $\mathfrak{R} L_{j}$ e $\mathfrak{I} L_{j}$,

$$
\operatorname{span}\left\{\left(\mathfrak{R L} \mathrm{L}_{\mathfrak{j}}\right)_{\mathrm{p}},\left(\mathfrak{I} \mathrm{L}_{\mathfrak{j}}\right)_{\mathfrak{p}} ; \mathfrak{j}=1, \ldots, \mathfrak{n}\right\}=\mathcal{V}_{\mathrm{p}}
$$

Este conjunto contém $2 n$ vetores, mas $\operatorname{dim} \nu_{p}=n$. Então é possível extrair $n$ deles que sejam linearmente independentes e gerem $V_{p}$. Denote-os por $\left(M_{1}\right)_{p}, \ldots,\left(M_{n}\right)_{p}$, onde os $M_{j}$ são alguns dos campos reais $\mathfrak{R} L_{j}$ e $\mathfrak{I} L_{j}$. Por continuidade, estes mesmos $M_{j}$ geram $\mathcal{V}$ em um aberto possivelmente menor em torno de $p$, como queríamos.

Teorema 1.39. Se $\mathcal{V}$ é uma estrutura $C R$ de posto $\mathrm{n}$, então $\mathrm{T}^{0}$ é um subfibrado de $\mathrm{T}^{*} \Omega$ de posto $\mathrm{N}-2 \mathrm{n}$. 
Devemos destacar que o posto de um subfibrado de $\mathrm{T} \Omega$ ou de $\mathrm{T}^{*} \Omega$ refere-se à dimensão real de suas fibras, pois estas são neste caso $\mathbb{R}$-espaços vetoriais. Ainda, nas hipóteses do teorema, temos $N-2 n \geq 0$, já que $\mathcal{V}_{p} \cap \overline{\mathcal{V}_{p}}=0$ por hipótese, implicando

$$
2 \mathrm{n}=\operatorname{dim}\left(\mathcal{V}_{\mathrm{p}}+\overline{\mathcal{V}_{\mathrm{p}}}\right) \leq \operatorname{dim} \mathbb{C} \mathrm{T}_{\mathrm{p}} \Omega=\mathrm{N}
$$

Demonstração de 1.39. Para cada $p \in \Omega$, seja $\mathcal{W}_{p}=\mathcal{V}_{p} \oplus \overline{\mathcal{V}_{p}}$, e seja

$$
\mathcal{W}=\bigcup_{p \in \Omega} \mathcal{W}_{p}
$$

A dimensão complexa de cada fibra $\mathcal{W}_{p}$ é $2 n$, e se $L_{1}, \ldots, L_{n}$ são os campos que geram $\mathcal{V}$ em torno de um dado $p$, então os campos $L_{1}, \ldots, L_{n}, \overline{L_{1}}, \ldots, \overline{L_{n}}$ geram $\mathcal{W}$ ali. Logo $\mathcal{W}$ é um subfibrado tangente, e ainda, é essencialmente real, dado que $\mathcal{W}_{\mathrm{p}}=\overline{\mathcal{W}_{\mathrm{p}}}$. Em particular, $\mathcal{W}^{\perp}$ é também essencialmente real, devido à proposição 1.23 .

Seja $p \in \Omega$ fixado. A proposição 1.38 permite afirmar que $\mathcal{W}^{\perp}$ é gerado sobre $\mathbb{C}$ em uma vizinhança $U \ni p$ por formas reais $\omega_{1}, \ldots, \omega_{d}$. Para cada $q \in U$, defina o $\mathbb{R}$-espaço vetorial

$$
A_{\mathrm{q}}=\operatorname{span}_{\mathbb{R}}\left\{\left(\omega_{1}\right)_{\mathrm{q}}, \ldots,\left(\omega_{\mathrm{d}}\right)_{\mathrm{q}}\right\}
$$

Vamos provar que $A_{q}=T_{q}^{0}$, e dessa forma $T^{0}$ será um subfibrado real.

Inicialmente observe que, como $\left(\omega_{j}\right)_{q} \in \mathcal{W}_{q}^{\perp}=\left(\mathcal{V}_{q} \oplus \overline{\mathcal{V}_{q}}\right)^{\perp}$, temos $\left(\omega_{j}\right)_{q}(v)=0$ para todo $v \in \mathcal{V}_{\mathrm{q}} ; \operatorname{logo} A_{\mathrm{q}} \subseteq \mathcal{V}_{\mathrm{q}}^{\perp}$. Ainda, sendo cada $\omega_{\mathrm{j}}$ real, e sendo $A_{\mathrm{q}}$ um espaço real, temos $A_{\mathrm{q}} \subseteq \mathrm{T}_{\mathrm{q}}^{*} \Omega$. Portanto $A_{\mathrm{q}} \subseteq \mathrm{T}_{\mathrm{q}}^{0}$.

Reciprocamente, seja $\xi \in \mathrm{T}_{\mathrm{q}}^{0}$. Então $\xi \in \mathcal{V}_{\mathrm{q}}^{\perp}$ e também $0=\overline{\xi(\nu)}=\bar{\xi}(\bar{v})=\xi(\bar{v})$ para cada $\nu \in \mathcal{V}_{\mathrm{q}}$, isto é, $\xi \in \overline{\mathcal{V}_{\mathrm{q}}^{\perp}}$. Logo $\xi \in \mathcal{W}_{\mathrm{q}}^{\perp}$. Então escreva

$$
\xi=\sum_{j=1}^{d} \alpha_{j}\left(\omega_{j}\right)_{q} \quad, \quad \alpha_{j} \in \mathbb{C}
$$

Sendo $\xi=\bar{\xi}$ e sendo os covetores $\left(\omega_{j}\right)_{q}$ linearmente independentes sobre $\mathbb{C}$, se tomarmos o conjugado na igualdade acima obteremos que $\alpha_{j} \in \mathbb{R}$. Então $\xi \in A_{q}$.

\subsection{Estruturas analíticas}

Assuma durante esta seção que $\Omega$ é uma variedade analítica, isto é, as funções de mudança de coordenadas são não apenas suaves, como também analíticas reais. Definiremos campos, formas, subfibrados etc. analíticos em $\Omega$ generalizando o que foi feito até então.

Um campo $L \in X(\Omega)$ é dito analítico real se $L\left(C^{\omega}(\Omega)\right) \subseteq C^{\omega}(\Omega)$ (aqui $C^{\omega}(\Omega)$ denota o espaço das funções analíticas em $\Omega$ ), ou, equivalentemente, se sempre que $L$ for dado em coordenadas locais na forma $L=\sum a_{j} \partial_{x_{j}}$, as funções $a_{j}$ forem analíticas. Uma forma 
$\omega \in \Lambda^{1}(\Omega)$ é dita analítica se $\omega(L) \in C^{\omega}(\Omega)$ para todo $L \in X(\Omega)$ analítico. Vale também uma equivalência com a representação local de $\omega$ em coordenadas, como para campos. Um subfibrado de $\mathbb{C} T \Omega$ (resp. $\mathbb{C T}^{*} \Omega$ ) é analítico se é gerado localmente por campos (resp. formas) analíticos.

Seja $L=\sum_{j=1}^{N} a_{j} \frac{\partial}{\partial x_{j}}$ um campo analítico dado em coordenadas locais $x_{1}, \ldots, x_{N}$ em um aberto $u \subseteq \Omega$. Fixada $u \in C^{\omega}(\Omega)$, é sabido que podemos estender as funções $a_{1}, \ldots, a_{N}, u$ a funções holomorfas $\tilde{a}_{1}, \ldots, \tilde{a}_{N}, \tilde{u}$ em um aberto $\Sigma \subseteq \mathbb{C}^{N}$ que contém $U$. Defina o campo holomorfo

$$
\tilde{\mathrm{L}}=\sum_{j=1}^{\mathrm{N}} \tilde{\mathrm{a}}_{j} \frac{\partial}{\partial z_{j}}
$$

Então temos

$$
\mathrm{Lu}=\left.(\tilde{\mathrm{L}} \tilde{u})\right|_{\mathrm{u}}
$$

De fato, se $x \in \mathrm{U}$,

$$
(\tilde{L} \tilde{u})(x)=\sum \tilde{a}_{j}(x) \frac{\partial \tilde{u}}{\partial z_{j}}(x)=\sum a_{j}(x) \frac{\partial \tilde{u}}{\partial x_{j}}(x)=\sum a_{j}(x) \frac{\partial u}{\partial x_{j}}(x)
$$

o que é decorrente do fato que a derivação complexa de uma função holomorfa coincide com a derivação com relação à coordenada correspondente à parte real (isto é, os campos $\partial_{x_{j}}$ e $\partial_{z_{j}}$ coincidem em $\left.\mathcal{O}(\Sigma)\right)$.

A importância de se estender um campo analítico de forma que valha a equação (1.40) reside no fato que campos holomorfos se comportam como análogos complexos dos campos reais, e por isso admitem propriedades úteis como o Teorema de Frobenius. Utilizaremos essa abordagem futuramente.

\subsection{Geradores locais}

Os teoremas seguintes, cujas demonstrações envolvem apenas Álgebra Linear elementar, fornecem uma representação canônica para os campos que geram um subfibrado tangente qualquer em torno de um ponto, bem como simplificações que ocorrem para os diferentes tipos de estruturas.

Lema 1.41. Seja $\mathrm{V}$ um subespaço vetorial de $\mathbb{C}^{\mathrm{N}}$, com $\operatorname{dim}_{\mathbb{C}} \mathrm{V}=\mathrm{m}$. Defina $\mathrm{V}^{0}=\mathrm{V} \cap \mathbb{R}^{\mathrm{N}}$ e denote sua dimensão real por $\mathrm{d}=\operatorname{dim}_{\mathbb{R}} \mathrm{V}^{0}$. Seja $\left\{\xi_{\mathrm{m}-\mathrm{d}+1}, \ldots, \xi_{\mathrm{m}}\right\}$ uma base para $\mathrm{V}^{0}$ sobre $\mathbb{R}$. Seja ainda $\mathrm{V}_{1}$ o subespaço de $\mathrm{V}$ tal que $\left(\mathrm{V}^{0}+i \mathrm{~V}^{0}\right) \oplus \mathrm{V}_{1}=\mathrm{V}$, e seja $\left\{\eta_{1}, \ldots, \eta_{v}\right\}$ uma base para $\mathrm{V}_{1}$ sobre $\mathbb{C}$ (note que $v=\mathrm{m}-\mathrm{d}$ ). Para $\mathrm{j}=1, \ldots, \mathrm{v}$, defina $\xi_{\mathrm{j}}, \lambda_{\mathrm{j}} \in \mathbb{R}$ por $\eta_{j}=\xi_{j}+i \lambda_{j}$. Nessas condições, temos:

- $\left\{\eta_{1}, \ldots, \eta_{v}, \xi_{v+1}, \ldots, \xi_{m}\right\}$ é uma base para $\mathrm{V}$ sobre $\mathbb{C}$; 
- o conjunto $\left\{\xi_{1}, \ldots, \xi_{m}, \lambda_{1}, \ldots, \lambda_{v}\right\}$ é $\mathbb{R}$-linearmente independente;

- $v+m \leq N$.

Demonstração: Para provar o primeiro item, basta completarmos $\left\{\xi_{v+1}, \ldots, \xi_{m}\right\}$, que é uma base para $\mathrm{V}^{0}+i \mathrm{~V}^{0}$ sobre $\mathbb{C}$, com a base de $\mathrm{V}_{1}$, obtendo assim uma base para $\mathrm{V}$.

Agora observe que, de acordo com o lema 1.36,

$$
\mathrm{V} \cap \overline{\mathrm{V}_{1}} \subseteq \mathrm{V} \cap \overline{\mathrm{V}}=\mathrm{V}^{0}+i \mathrm{~V}^{0}=\overline{\mathrm{V}^{0}+i \mathrm{~V}^{0}}
$$

Logo,

$$
\mathrm{V} \cap \overline{\mathrm{V}_{1}} \subseteq \overline{\mathrm{V}_{1}} \cap \overline{\mathrm{V}^{0}+i \mathrm{~V}^{0}}=\overline{\mathrm{V}_{1} \cap\left(\mathrm{V}^{0}+i \mathrm{~V}^{0}\right)}=\{0\}
$$

e portanto $\mathrm{V}^{0} \cap \overline{\mathrm{V}_{1}}=\mathrm{V}_{1} \cap \overline{\mathrm{V}_{1}}=\{0\}$, uma vez que $\mathrm{V}^{0}, \mathrm{~V}_{1} \subseteq \mathrm{V}$. Junte-se a isso o fato que também $V^{0} \cap V_{1}=\{0\}$. Então qualquer reunião de uma $\mathbb{C}$-base de $V_{1}$, uma $\mathbb{C}$-base de $\overline{V_{1}} \mathrm{e}$ uma $\mathbb{C}$-base de $\mathrm{V}^{0}$ será linearmente independente sobre $\mathbb{C}$ :

$$
\left\{\eta_{1}, \ldots, \eta_{v}, \overline{\eta_{1}}, \ldots, \overline{\eta_{v}}, \xi_{v+1}, \ldots, \xi_{m}\right\} \text { é l.i. sobre } \mathbb{C}
$$

Tomando partes reais e imaginárias:

$$
\left\{\xi_{1}, \ldots, \xi_{v}, \lambda_{1}, \ldots, \lambda_{v}, \xi_{v+1}, \ldots, \xi_{m}\right\} \text { é l.i. sobre } \mathbb{R}
$$

Isto prova o segundo item. Quanto ao terceiro, é suficiente notar que o conjunto l.i. obtido em (1.42) tem $2 v+m-(v+1)+1=v+$ m elementos.

Teorema 1.43. Seja $\mathcal{V}$ um subfibrado tangente de posto n. Então, dado $p \in \Omega$, existem inteiros não-negativos $\mathrm{h}, \mathrm{d}, \mathrm{r}$ satisfazendo $2 \mathrm{~h}+\mathrm{d}+\mathrm{r}=\mathrm{N}$ (onde $\mathrm{N}=\operatorname{dim} \Omega$ ) e $\mathrm{h}+\mathrm{r}=\mathrm{n}$, e existem coordenadas locais

$$
x_{1}, \ldots, x_{h}, y_{1}, \ldots, y_{h}, s_{1}, \ldots, s_{d}, t_{1}, \ldots, t_{r}
$$

com centro em $p$ (isto é, $p$ corresponde a $x_{j}=y_{j}=s_{k}=t_{l}=0$ ) tais que $v$ é gerado em torno de p por campos da forma

$$
\begin{aligned}
& \mathrm{L}_{\mu}=\frac{\partial}{\partial \overline{z_{\mu}}}+\sum_{j=1}^{\nu} \mathrm{a}_{\mu j} \frac{\partial}{\partial z_{j}}+\sum_{k=1}^{d} \mathrm{c}_{\mu k} \frac{\partial}{\partial s_{k}}, \mu=1, \ldots, v \\
& \tilde{\mathrm{L}}_{\mu}=\frac{\partial}{\partial \mathrm{t}_{\mu}}+\sum_{j=1}^{v} \tilde{\mathrm{a}}_{\mu j} \frac{\partial}{\partial z_{j}}+\sum_{\mathrm{k}=1}^{\mathrm{d}} \tilde{\mathrm{c}}_{\mu \mathrm{k}} \frac{\partial}{\partial s_{k}} \quad, \quad \mu=1, \ldots, r
\end{aligned}
$$

Aqui, $\mathrm{a}_{\mu \mathrm{j}}, \tilde{\mathrm{a}}_{\mu \mathrm{j}}, \mathrm{c}_{\mu \mathrm{k}}, \tilde{\mathrm{c}}_{\mu \mathrm{k}} \in \mathrm{C}^{\infty}(\Omega)$ são funções que se anulam em $\mathrm{p}$, e estabelecemos as coordenadas

$$
\begin{array}{ll}
z_{\mu}=x_{\mu}+i y_{\mu} & , \quad \mu=1, \ldots, h \\
\overline{z_{\mu}}=x_{\mu}-i y_{\mu} & , \quad \mu=1, \ldots, h
\end{array}
$$


e os campos

$$
\frac{\partial}{\partial z_{\mu}}=\frac{1}{2}\left(\frac{\partial}{\partial x_{\mu}}-i \frac{\partial}{\partial y_{\mu}}\right) \quad e \quad \frac{\partial}{\partial \overline{z_{\mu}}}=\frac{1}{2}\left(\frac{\partial}{\partial x_{\mu}}+i \frac{\partial}{\partial y_{\mu}}\right)
$$

A definição dos campos $\partial_{z_{\mu}}$ e $\partial_{\overline{z_{\mu}}}$ é como acima para que estes sejam os duais das formas $\mathrm{d} z_{\mu}$ e $\mathrm{d} \overline{z_{\mu}}$, que ficam definidas a partir das equações (1.44). Dessa maneira, eles serão também os campos correspondentes a cada coordenada $z_{\mu}$ e $\overline{z_{\mu}}$, de forma análoga à construção feita em (1.4). Novamente, ressaltamos que $z_{\mu}$ e $\overline{z_{\mu}}$ não são de fato coordenadas reais em $\Omega$, mas, conforme veremos, tudo funcionará como se fossem, e será conveniente trabalhar com elas a partir daqui.

Demonstração de 1.43. Aplique o lema $1.41 \mathrm{com} \mathcal{V}_{p}^{\perp}$ no lugar de V. Conclui-se a existência de uma $\mathbb{C}$-base de $\mathcal{V}_{p}^{\perp}$,

$$
\left\{\eta_{1}, \ldots, \eta_{v}, \xi_{v+1}, \ldots, \xi_{m}\right\} \quad, \quad \eta_{j}=\xi_{j}+i \lambda_{j}, j=1, \ldots, v
$$

satisfazendo:

- $\mathrm{m}=\operatorname{dim}_{\mathbb{C}} \mathcal{V}_{\mathrm{p}}^{\perp}$

- $\mathrm{d}=\mathrm{v}+\mathrm{m}=\operatorname{dim}_{\mathbb{R}} \mathrm{T}_{\mathrm{p}}^{0} \leq \mathrm{N}$

- $\left\{\xi_{v+1}, \ldots, \xi_{m}\right\}$ é base para $T_{p}^{0}$ sobre $\mathbb{R}$;

- $\left(T_{p}^{0}+i T_{p}^{0}\right) \oplus \operatorname{span}\left\{\eta_{1}, \ldots, \eta_{v}\right\}=\mathcal{V}_{p}^{\perp}$

- $\left\{\xi_{1}, \ldots, \xi_{m}, \lambda_{1}, \ldots, \lambda_{v}\right\}$ é linearmente independente sobre $\mathbb{R}$.

Devido a este último item, é possível escolher uma carta centrada em p com coordenadas

$$
x_{1}, \ldots, x_{v}, y_{1}, \ldots, y_{v}, s_{1}, \ldots, s_{d}, t_{1}, \ldots, t_{r}
$$

de tal forma que

$$
\begin{gathered}
\left(d x_{j}\right)_{p}=\xi_{j},\left(d y_{j}\right)_{p}=\lambda_{j} \quad, \quad j=1, \ldots, v \\
\left(d s_{k}\right)_{p}=\xi_{v+k} \quad, \quad k=1, \ldots, d
\end{gathered}
$$

Observe que $r=N-2 v-d$ e assim $v+r=n$. Se definirmos as coordenadas $z_{j}$ e $\overline{z_{j}}$ como no enunciado, então todo campo $L \in \mathcal{X}(\Omega)$ se escreve nessa carta na forma

$$
L=\sum_{j=1}^{v} A_{j} \frac{\partial}{\partial z_{j}}+\sum_{j=1}^{v} B_{j} \frac{\partial}{\partial \overline{z_{j}}}+\sum_{k=1}^{d} C_{k} \frac{\partial}{\partial s_{k}}+\sum_{l=1}^{r} D_{l} \frac{\partial}{\partial t_{l}}
$$


Mas se $L$ for seção de $\mathcal{V}$, todo vetor cotangente $\lambda \in \mathcal{V}_{p}^{\perp}$ anula $L_{p}$, e assim

$$
\begin{array}{r}
0=\left(d\left(x_{j}+i y_{j}\right)\right)_{p}\left(L_{p}\right)=2\left(d z_{j}\right)_{p}\left(L_{p}\right)=2 A_{j}(p), \quad j=1, \ldots, v \\
0=\left(d s_{k}\right)_{p}\left(L_{p}\right)=C_{k}(p), \quad k=1, \ldots, d
\end{array}
$$

Sejam $\mathrm{L}_{1}, \ldots, \mathrm{L}_{v}, \tilde{\mathrm{L}}_{1}, \ldots, \tilde{\mathrm{L}}_{r}$ campos que geram $v$ perto de $p$, e que expressos nas coordenadas acima sejam

$$
\begin{aligned}
& \mathrm{L}_{\mu}=\sum_{j=1}^{v} \mathrm{a}_{\mu j} \frac{\partial}{\partial z_{j}}+\sum_{p=1}^{v} \mathrm{~b}_{\mu j} \frac{\partial}{\partial \overline{z_{j}}}+\sum_{k=1}^{\mathrm{d}} \mathrm{c}_{\mu k} \frac{\partial}{\partial s_{k}}+\sum_{l=1}^{r} \mathrm{~d}_{\mu l} \frac{\partial}{\partial t_{l}}, \mu=1, \ldots, v \\
& \tilde{\mathrm{L}}_{\mu}=\sum_{j=1}^{v} \tilde{\mathrm{a}}_{\mu j} \frac{\partial}{\partial z_{j}}+\sum_{j=1}^{v} \tilde{b}_{\mu j} \frac{\partial}{\partial \overline{z_{j}}}+\sum_{k=1}^{\mathrm{d}} \tilde{c}_{\mu k} \frac{\partial}{\partial s_{k}}+\sum_{l=1}^{r} \tilde{d}_{\mu l} \frac{\partial}{\partial t_{l}}, \quad \mu=1, \ldots, r
\end{aligned}
$$

onde $a_{\mu j}, b_{\mu j}, c_{\mu k}, d_{\mu l}, \tilde{a}_{\mu j}, \tilde{b}_{\mu j}, \tilde{c}_{\mu k}, \tilde{d}_{\mu l}$ são funções suaves que, de acordo com o observado acima, satisfazem

$$
a_{\mu j}(p), c_{\mu k}(p), \tilde{a}_{\mu j}(p), \tilde{c}_{\mu k}(p)=0 \quad \text { para todos } \mu, j, k, l
$$

Sejam as matrizes

$$
\begin{array}{llll}
A=\left(a_{\mu j}\right)_{v \times v} & B=\left(b_{\mu j}\right)_{v \times v} & C=\left(c_{\mu k}\right)_{v \times d} & D=\left(d_{\mu l}\right)_{v \times r} \\
\tilde{A}=\left(\tilde{a}_{\mu j}\right)_{r \times v} & \tilde{B}=\left(\tilde{b}_{\mu j}\right)_{r \times v} & \tilde{C}=\left(\tilde{c}_{\mu k}\right)_{r \times d} & \tilde{D}=\left(\tilde{d}_{\mu l}\right)_{r \times r}
\end{array}
$$

Se definirmos

$$
\begin{gathered}
\mathrm{L}=\left(\mathrm{L}_{1}, \ldots, \mathrm{L}_{v}, \tilde{\mathrm{L}}_{1}, \ldots, \tilde{\mathrm{L}}_{\mathrm{r}}\right)^{\mathrm{T}} \\
\nabla=\left(\partial_{z_{1}}, \ldots, \partial_{z_{v}}, \partial_{\overline{z_{1}}}, \ldots, \partial_{\overline{z_{v}}}, \partial_{s_{1}}, \ldots, \partial_{s_{\mathrm{d}}}, \partial_{t_{1}}, \ldots, \partial_{\mathrm{t}_{r}}\right)^{\top} \\
M=\left(\begin{array}{c|c|c|c}
\mathrm{A} & \mathrm{B} & \mathrm{C} & \mathrm{D} \\
\hline \tilde{A} & \tilde{\mathrm{B}} & \tilde{\mathrm{C}} & \tilde{\mathrm{D}}
\end{array}\right)_{n \times N}
\end{gathered}
$$

então $L=M \nabla$, e o posto de $M(p)$ é o mesmo que o da estrutura $\mathcal{V}$, isto é, n. Seja agora

$$
P=\left(\begin{array}{l|l}
B & D \\
\hline \tilde{B} & \tilde{D}
\end{array}\right)_{n \times n}
$$

Devido a (1.45) e ao fato do posto de $M(p)$ ser $n$, a matriz $P(p)$ é necessariamente invertível, e por continuidade $P(q)$ também é invertível para q numa vizinhança $W$ de $p$. Em $W$, defina $n$ campos de vetores linearmente independentes $L^{\sharp}=\left(L_{1}^{\sharp}, \ldots, L_{v}^{\sharp}, \tilde{L}_{1}^{\sharp}, \ldots, \tilde{L}_{r}^{\sharp}\right)^{\top}$ por

$$
\mathrm{L}^{\sharp}=\mathrm{P}^{-1} \mathrm{~L}=\mathrm{P}^{-1} M \nabla=\left(\begin{array}{c|c|c|c}
A_{v \times v}^{\sharp} & \mathrm{I}_{v \times v} & \mathrm{C}_{v \times \mathrm{d}}^{\sharp} & O_{v \times \mathrm{r}} \\
\hline \tilde{A}_{v \times v}^{\sharp} & O_{r \times v} & \tilde{C}_{v \times d}^{\sharp} & \mathrm{I}_{\mathrm{r} \times \mathrm{r}}
\end{array}\right) \nabla
$$


Os campos $L^{\sharp}$ são então escritos na forma

$$
\begin{aligned}
& \mathrm{L}_{\mu}^{\sharp}=\frac{\partial}{\partial \overline{z_{\mu}}}+\sum_{j=1}^{v} a_{\mu j}^{\sharp} \frac{\partial}{\partial z_{j}}+\sum_{k=1}^{d} c_{\mu k}^{\sharp} \frac{\partial}{\partial s_{k}}, \mu=1, \ldots, v \\
& \tilde{L}_{\mu}^{\sharp}=\frac{\partial}{\partial t_{\mu}}+\sum_{j=1}^{v} \tilde{a}_{\mu j}^{\sharp} \frac{\partial}{\partial z_{j}}+\sum_{k=1}^{d} \tilde{c}_{\mu k}^{\sharp} \frac{\partial}{\partial s_{k}}, \mu=1, \ldots, r
\end{aligned}
$$

e geram $\mathcal{V}$ por serem linearmente independentes.

Corolário 1.46. Com as notações do teorema acima, se $\mathcal{V}$ é uma estrutura:

- elíptica em $\mathrm{p}$, então $\mathrm{d}=0$ e $\mathrm{v}=\mathrm{N}-\mathrm{n}$;

- Cauchy-Riemann em $\mathrm{p}$, então $\mathrm{r}=0$;

- complexa em $\mathrm{p}$, então $\mathrm{d}=\mathrm{r}=0$ e $\mathrm{v}=\mathrm{N}-\mathrm{n}$.

- essencialmente real em $\mathrm{p}$, então $\mathrm{d}=\mathrm{m}, \boldsymbol{v}=0$ e $\mathrm{r}=\mathrm{n}$.

\subsection{Estruturas localmente integráveis}

Definição 1.47. Um subfibrado tangente $\mathcal{V}$ de posto $n$ é dito ser uma estrutura localmente integrável se em torno de cada $p \in \Omega$ existe uma vizinhança $\mathrm{U}$ onde estejam definidas $\mathrm{m}=\mathrm{N}-\mathrm{n}$ integrais primeiras da estrutura, isto é, funções $\mathrm{Z}_{1}, \ldots, \mathrm{Z}_{\mathrm{m}} \in \mathrm{C}^{\infty}(\mathrm{U})$ tais que

$$
\operatorname{span}\left\{\left(d Z_{1}\right)_{q}, \ldots,\left(d Z_{m}\right)_{q}\right\}=V_{q}^{\perp} \quad, \quad q \in U
$$

Portanto, se $\mathcal{V}$ é gerada por campos linearmente independentes $L_{1}, \ldots, L_{n}$, então será localmente integrável se e somente se pudermos encontrar $m$ soluções locais $Z_{1}, \ldots, Z_{m}$ do sistema $L_{j} Z=0, j=1, \ldots, n$, tais que $\left\{d Z_{1}, \ldots, d Z_{m}\right\}$ seja linearmente independente. Dessa forma, uma estrutura localmente integrável de posto $n$ é aquela que admite o número esperado $\mathrm{m}=\mathrm{N}-\mathrm{n}$ de soluções independentes.

Lema 1.48. Se V é um subfibrado tangente, então

$$
\mathrm{dg} \text { é seção de } \mathcal{V}^{\perp} \Longleftrightarrow \mathrm{Lg}=0 \text { para todo } \mathrm{L} \text { seção de } \mathcal{V}
$$

para toda $\mathrm{g} \in \mathrm{C}^{\infty}(\Omega)$. 
Demonstração: Se dg e L são seções de $\mathcal{V}^{\perp}$ e $\mathcal{V}$, nessa ordem, então para todo $p \in \Omega$ tem-se $\operatorname{Lg}(p)=d g(L)(p)=(d g)_{p}\left(L_{p}\right)=0$, já que $(d g)_{p} \in \mathcal{V}_{p}^{\perp}$ e $L_{p} \in \mathcal{V}_{p}$. Reciprocamente, suponha que toda seção de $\mathcal{V}$ anula $g$, e tome $p \in \Omega$ e $v \in \mathcal{V}_{p}$. Queremos provar que $(d g)_{p}(v)=0$. E de fato, sabemos que existe uma seção $L$ tal que $L_{p}=v$, e portanto $(\mathrm{dg})_{\mathfrak{p}}(v)=(\mathrm{dg})_{\mathfrak{p}}\left(\mathrm{L}_{\mathrm{p}}\right)=\operatorname{Lg}(\mathrm{p})=0$.

Teorema 1.49. Toda estrutura localmente integrável é involutiva.

Demonstração: Sejam L, $M$ duas seções de $\mathcal{V}$ sobre um aberto $W \subseteq \Omega$, e tome $p \in W$. Queremos demonstrar que $[L, M]_{p} \in \mathcal{V}_{p}$. Seja $U \subseteq W$ uma vizinhança aberta de $p$ onde estejam definidas integrais primeiras $Z_{1}, \ldots, Z_{m} \in C^{\infty}(U)$. Em particular, $d Z_{1}, \ldots, d Z_{m}$ são seções de $\mathcal{V}^{\perp}$ sobre $U$ e geram $\mathcal{V}_{q}^{\perp}$ para todo $q \in U$. Pelo lema 1.48, temos $L\left(Z_{k}\right)=$ $M\left(Z_{k}\right)=0$ em $U$ para $k=1, \ldots, m$; logo também $[L, M]\left(Z_{k}\right)=0$. Agora tome $g \in C^{\infty}(U)$ arbitrária tal que dg seja seção de $\mathcal{V}^{\perp}$ sobre $U$. Se q é qualquer ponto de $U$, existem escalares $a_{1}, \ldots, a_{m}$ tais que

$$
(d g)_{q}=\sum_{k=1}^{m} a_{k}\left(d Z_{k}\right)_{q}
$$

Portanto,

$$
[L, M] g(q)=(d g)_{q}\left([L, M]_{q}\right)=\sum_{k=1}^{m} a_{k}\left(d Z_{k}\right)_{q}\left([L, M]_{q}\right)=\sum_{k=1}^{m} a_{k}[L, M] Z_{k}(q)=0
$$

Novamente pelo lema 1.48, concluímos que [L, M] é seção de $V$ sobre $\mathrm{U}$, o que prova que $[\mathrm{L}, \mathrm{M}]_{\mathrm{p}} \in \mathcal{V}_{\mathrm{p}}$, como queríamos.

Exemplo 1.50. Considere o operador de Mizohata $M=\partial_{t}-i t \partial_{x}$ em $\mathbb{R}^{2}$. Seja $Z(t, x)=$ $x+(\mathfrak{i} / 2) \mathrm{t}^{2}$. Então $\mathrm{dZ}=\mathrm{d} x+\mathrm{idt} \neq 0$ em $\mathbb{R}^{2}, e$

$$
M Z=\partial_{t}\left(i \frac{t^{2}}{2}\right)-i t \partial_{x}(x)=i t-i t=0
$$

Logo a estrutura de Mizohata é localmente integrável. Na verdade poderíamos excluir o advérbio localmente, já que Z é uma integral primeira global neste caso.

Teorema 1.51. Toda estrutura involutiva essencialmente real é localmente integrável.

Demonstração: Seja $\mathcal{V}$ involutiva essencialmente real e seja $p \in \Omega$. Pela proposição $1.38, V$ é gerada por campos reais em torno de $p$. Então podemos aplicar o Teorema de Frobenius para encontrar uma carta $(U, x)$ contendo $p$ onde $V$ seja gerada por $\partial_{x_{1}}, \ldots, \partial_{x_{n}}$. Em $U$, as funções $Z_{k}=x_{n+k}, k=1, \ldots, m$, se qualificam como integrais primeiras para $V$.

Teorema 1.52. Toda estrutura involutiva analítica é localmente integrável. 
Demonstração: O princípio aqui é o mesmo que no teorema anterior: aplicar o Teorema de Frobenius. Seja $\mathcal{V}$ involutiva e analítica e seja $p \in \Omega$. Existem campos analíticos $\mathrm{L}_{1}, \ldots, \mathrm{L}_{n}$ que geram $V$ em uma vizinhança $W$ de $p$. Para simplificar a notação, podemos assumir que $W=\mathbb{R}^{N}$ e $p=0$, e assim

$$
L_{j}=\sum_{j=1}^{N} a_{j k} \frac{\partial}{\partial x_{k}} \quad, \quad j=1, \ldots, n
$$

para certas funções analíticas $a_{j k}$. Também sabemos que existem funções $\lambda_{\alpha \beta}^{\gamma}$, a princípio suaves, tais que

$$
\left[\mathrm{L}_{\alpha}, \mathrm{L}_{\beta}\right]=\sum_{\gamma=1}^{\mathrm{n}} \lambda_{\alpha, \beta}^{\gamma} \mathrm{L}_{\gamma}, \quad \alpha, \beta=1, \ldots, \mathrm{n}
$$

Porém, se repetirmos a demonstração do teorema 1.13, veremos que na verdade $\lambda_{\alpha, \beta}^{\gamma}$ são analíticas. Então seja $U \subseteq \mathbb{C}^{N}$ aberto contendo a origem onde todas as funções $a_{j k}$ e $\lambda_{\alpha \beta}^{\gamma}$ possam ser estendidas a funções holomorfas $\tilde{a}_{j k}$ e $\tilde{\lambda}_{\alpha \beta}^{\gamma}$, e defina

$$
\tilde{\mathrm{L}}_{j}=\sum_{j=1}^{\mathrm{N}} \tilde{\mathrm{a}}_{j k} \frac{\partial}{\partial z_{k}} \quad, \quad j=1, \ldots, n
$$

Como o campo holomorfo

$$
\left[\tilde{\mathrm{L}}_{\alpha}, \tilde{\mathrm{L}}_{\beta}\right]-\sum_{\gamma=1}^{\mathrm{n}} \tilde{\lambda}_{\alpha \beta}^{\gamma} \tilde{\mathrm{L}}_{\gamma}
$$

é nulo em $\mathrm{U} \cap \mathbb{R}^{\mathrm{N}}$, é também nulo em $\mathrm{U}$. Assim, os campos $\tilde{\mathrm{L}}_{1}, \ldots, \tilde{\mathrm{L}}_{n}$ geram uma estrutura involutiva holomorfa $\mathcal{W}$ em $\mathcal{U}$. Pela versão holomorfa do Teorema de Frobenius, existe um aberto $V$ em torno de $0 \in \mathbb{C}^{N}$ onde $\mathcal{W}$ é gerada por $\partial_{z_{1}}, \ldots, \partial_{z_{n}}$, e ali as funções $W_{k}=z_{n+k}$, $k=1, \ldots, m$, se qualificam como integrais primeiras para $\mathcal{W}$. Devido às observações feitas ao final da seção 1.9 , as funções analíticas $Z_{k}=\left.W_{k}\right|_{\mathbb{R}^{N}}$ são integrais primeiras para $\mathcal{V}$ em $V \cap \mathbb{R}^{N}$.

Nosso próximo passo é encontrar geradores locais canônicos de $\mathcal{V}$ e $\mathcal{V}^{\perp}$ em torno de $p \in \Omega$ para uma estrutura localmente integrável $\mathcal{V}$, e então analisar o caso que nos será de interesse futuramente: quando $V$ é uma estrutura analítica.

Teorema 1.53. Seja $V$ localmente integrável de posto n. Seja $p \in \Omega$, e denote por $\mathrm{d}$ a dimensão real de $\mathrm{T}_{\mathrm{p}}^{0}$ e por $\mathrm{m}$ o coposto $\mathrm{N}-\mathrm{n}$. Então existe um sistema de coordenadas local centrado em $\mathrm{p}$

$$
x_{1}, \ldots, x_{v}, y_{1}, \ldots, y_{v}, s_{1}, \ldots, s_{d}, t_{1}, \ldots, t_{r}
$$

onde $v+\mathrm{d}=\mathrm{m}$ e $\mathrm{v}+\mathrm{r}=\mathrm{n}$, e existem funções suaves $\phi_{1}, \ldots, \phi_{\mathrm{d}}$, a valores reais, definidas em uma vizinhança de $\mathrm{p}$ e satisfazendo

$$
\phi_{k}(p)=0 \quad, \quad d \phi_{k}(p)=0, \quad k=1, \ldots, d
$$


tais que as diferenciais das funções suaves

$$
\begin{aligned}
z_{j}(x, y, s, t)=x_{j}+i y_{j} & , \quad j=1, \ldots, v \\
W_{k}(x, y, s, t)=s_{k}+i \phi_{k}(x, y, s, t) & , \quad k=1, \ldots, d
\end{aligned}
$$

geram $\mathcal{V}^{\perp}$ em torno de p. Ainda,

$$
\operatorname{span}\left\{d s_{1}(p), \ldots, d s_{d}(p)\right\}=T_{p}^{0}
$$

Demonstração: Por hipótese, existem $U \ni p$ aberto e $G_{1}, \ldots, G_{m} \in C^{\infty}(U)$ tais que $\mathrm{dG}_{1}, \ldots, \mathrm{dG}_{\mathrm{m}}$ geram $\mathcal{V}^{\perp}$ em $\mathrm{U}$. Aplicamos mais uma vez o lema $1.41 \mathrm{com} V=\mathcal{V}_{\mathrm{p}}^{\perp}$. Se $\left\{\eta_{1}, \ldots, \eta_{v}, \xi_{v+1}, \ldots, \xi_{m}\right\}$ é a base de $\mathcal{V}_{p}^{\perp}$ tal como dada naquele lema, com $v=m-d$ e $\mathrm{d}=\operatorname{dim}_{\mathbb{R}} \mathrm{T}_{\mathrm{p}}^{0}$, existem números $c_{j k} \in \mathbb{C}, j, k=1, \ldots, \mathrm{m}$, tais que a matriz $\left(c_{j k}\right)$ é invertível e

$$
\begin{aligned}
& \eta_{j}=\sum_{k=1}^{m} c_{j k} d_{G_{k}}(p), \quad j=1, \ldots, v \\
& \xi_{j}=\sum_{k=1}^{m} c_{j k} d G_{k}(p), \quad j=v+1, \ldots, m
\end{aligned}
$$

Defina em $U$ as funções suaves

$$
\begin{array}{cl}
Z_{j}=\sum_{k=1}^{m} c_{j k}\left(G_{k}-G_{k}(p)\right) \quad, \quad j=1, \ldots, v \\
W_{l}=\sum_{k=1}^{m} c_{v+l, k}\left(G_{k}-G_{k}(p)\right), \quad l=1, \ldots, m-v=d
\end{array}
$$

Estas funções se anulam em $p$, e suas diferenciais continuam gerando $\mathcal{V}^{\perp}$ em $\mathrm{U}$. Agora, devido ao segundo item do lema 1.41 , podemos definir coordenadas reais $x_{j}, y_{j}, s_{l}$ em uma vizinhança aberta $\mathrm{V} \ni \mathrm{p}$ possivelmente menor que $\mathrm{U}$, dadas por

$$
x_{j}=\mathfrak{R} Z_{j} \quad, \quad y_{j}=\mathfrak{I} Z_{j} \quad, \quad s_{l}=\mathfrak{R} W_{l}
$$

Finalmente, complete esse sistema de coordenadas com $t_{1}, \ldots, t_{r}$ (onde $r=N-2 v-d$ ) que se anulem em $p$, de forma que

$$
\left\{d x_{1}, \ldots, d x_{v}, d y_{1}, \ldots, d y_{v}, d s_{1}, \ldots, d s_{d}, d t_{1}, \ldots, d t_{r}\right\}
$$

seja linearmente independente em $V$. Basta agora definirmos $\phi_{k}=\mathfrak{I} W_{k}, k=1, \ldots, d$. Note que $\phi_{k}(p)=0$, porque $W_{k}(p)=0$, e que $d \phi_{k}(p)=0$, pois $d W_{k}(p)=\xi_{v+k}$ é real. 
Teorema 1.54. Seja $\mathcal{V}$ localmente integrável de posto $n$. Seja $p \in \Omega$ e seja $(x, y, s, t)$ a carta local dada pelo teorema anterior. Defina novas coordenadas $z_{j}=x_{j}+i y_{j}=Z_{j}$ $e \overline{z_{j}}=x_{j}-i y_{j}$, e considere a carta centrada em $p$ dada por

$$
z_{1}, \ldots, z_{v}, \overline{z_{1}}, \ldots, \overline{z_{v}}, s_{1}, \ldots, s_{d}, t_{1}, \ldots, t_{r}
$$

Então em torno de p existem campos linearmente independentes

$$
\mathrm{L}_{1}^{b}, \ldots, \mathrm{L}_{v}^{b}, \mathrm{~L}_{1}, \ldots, \mathrm{L}_{v}, \mathrm{M}_{1}, \ldots, \mathrm{M}_{\mathrm{d}}, \tilde{\mathrm{L}}_{1}, \ldots, \tilde{\mathrm{L}}_{\mathrm{r}}
$$

que geram o fibrado tangente, comutam dois a dois e são dados no formato

$$
\begin{aligned}
L_{j}^{b}=\frac{\partial}{\partial z_{j}}+\sum_{\rho=1}^{d} a_{j \rho}^{b} \frac{\partial}{\partial s_{\rho}} & L_{j}=\frac{\partial}{\partial \overline{z_{j}}}+\sum_{\rho=1}^{d} a_{j \rho} \frac{\partial}{\partial s_{\rho}} \\
M_{k}=\sum_{\rho=1}^{d} \mu_{k \rho} \frac{\partial}{\partial s_{\rho}} & \tilde{L}_{l}=\frac{\partial}{\partial t_{l}}+\sum_{\rho=1}^{d} \tilde{a}_{l \rho} \frac{\partial}{\partial s_{\rho}}
\end{aligned}
$$

onde $\mathrm{a}_{\mathrm{j} \rho}^{b}, \mathrm{a}_{\mathrm{j} \rho}, \mu_{\mathrm{k} \rho}, \tilde{\mathrm{a}}_{\mathrm{l} \rho}$ são funções suaves, tais que $\mathcal{V}$ é gerada por $\mathrm{L}_{1}, \ldots, \mathrm{L}_{v}, \tilde{\mathrm{L}}_{1}, \ldots, \tilde{\mathrm{L}}_{\mathrm{r}}$, e tais que a base dual da base

$$
\left\{d z_{1}, \ldots, d z_{v}, d \overline{z_{1}}, \ldots, d \overline{z_{v}}, d W_{1}, \ldots, d W_{d}, d t_{1}, \ldots, d t_{r}\right\}
$$

de $\mathbb{C T}^{*} \mathrm{U} e ́$

$$
\left\{\mathrm{L}_{1}^{b}, \ldots, \mathrm{L}_{v}^{b}, \mathrm{~L}_{1}, \ldots, \mathrm{L}_{v}, \mathrm{M}_{1}, \ldots, \mathrm{M}_{\mathrm{d}}, \tilde{\mathrm{L}}_{1}, \ldots, \tilde{\mathrm{L}}_{\mathrm{r}}\right\}
$$

Demonstração: Abreviaremos a lista $\left(z_{1}, \ldots, z_{v}, \overline{z_{1}}, \ldots, \overline{z_{v}}, s_{1}, \ldots, s_{d}, t_{1}, \ldots, t_{r}\right)$ escrevendo apenas $(z, s, t)$. Primeiramente, é possível encontrar, próximo a $p$, funções suaves $\mu_{k \rho}$, $k, \rho=1, \ldots, d$, tais que os campos

$$
M_{k}=\sum_{\rho=1}^{d} \mu_{k \rho}(z, s, t) \frac{\partial}{\partial s_{\rho}} \quad, \quad k=1, \ldots, d
$$

satisfaçam $M_{k} W_{\rho}=\delta_{k \rho}$. De fato, a matriz Jacobiana $\left(\partial_{s_{\rho}} W_{k}\right)_{d \times d}$ é igual à identidade em $p$, portanto é invertível em uma vizinhança de $p$, e basta definirmos as funções $\mu_{k \rho}$ como os elementos de sua inversa.

Sejam agora

$$
\begin{array}{ll}
\mathrm{L}_{\mathrm{j}}=\frac{\partial}{\partial \overline{z_{\mathrm{j}}}}-i \sum_{\mathrm{k}=1}^{\mathrm{d}} \frac{\partial \phi_{\mathrm{k}}}{\partial \overline{z_{\mathrm{j}}}}(z, s, \mathrm{t}) M_{\mathrm{k}}, & \mathrm{j}=1, \ldots, v \\
\tilde{\mathrm{L}}_{\mathrm{l}}=\frac{\partial}{\partial \mathrm{t}_{\mathrm{l}}}-\mathrm{i} \sum_{\mathrm{k}=1}^{\mathrm{d}} \frac{\partial \phi_{\mathrm{k}}}{\partial \mathrm{t}_{\mathrm{l}}}(z, s, \mathrm{t}) \mathrm{M}_{\mathrm{k}}, \quad l=1, \ldots, r
\end{array}
$$


onde as $\phi_{k}$ são dadas como no teorema anterior. Trata-se de campos l.i., tendo-se em vista que a primeira parcela é única em cada um e não aparece na segunda parcela. Mas também geram $v$ em U. De fato, temos:

$$
\begin{aligned}
\mathrm{L}_{\mathrm{j}} \mathrm{Z}_{\mathfrak{u}}=\partial_{\overline{z_{j}}} \mathrm{Z}_{\mathfrak{u}}-0=0 & \tilde{\mathrm{L}}_{\mathrm{l}} \mathrm{Z}_{\mathfrak{u}}=0-0=0 \\
\mathrm{~L}_{\mathrm{j}} \mathrm{W}_{\rho}=\partial_{\overline{z_{j}}} W_{\rho}-i \partial_{\overline{z_{j}}} \phi_{\rho}=0 & \tilde{\mathrm{L}}_{\mathrm{l}} \mathrm{W}_{\rho}=\partial_{\mathrm{t}_{\mathrm{l}}} W_{\rho}-i \partial_{\mathrm{t}_{l}} \phi_{\rho}=0
\end{aligned}
$$

Então, porque $d\left(Z_{u}\right), d\left(W_{\rho}\right)$ geram $\mathcal{V}^{\perp}$ em $U$, concluímos que cada $L_{j}$, $\tilde{L}_{l}$ é seção de $\mathcal{V}$ sobre $U$ (vide lema 1.48), e por estarem em quantidade certa $(\nu+r=n)$, esses campos geram $\mathcal{V}$ em $\mathrm{U}$, como havíamos afirmado.

Por fim, defina os seguintes campos em U:

$$
L_{j}^{b}=\frac{\partial}{\partial z_{j}}-i \sum_{k=1}^{d} \frac{\partial \phi_{k}}{\partial z_{j}}(z, s, t) M_{k} \quad, \quad j=1, \ldots, v
$$

Cálculos diretos tais como acima provam a afirmação sobre a dualidade de bases. Dessa afirmação também segue que todos os campos $L_{j}^{b}, L_{j}, M_{k}, \tilde{L}_{l}$ comutam dois a dois. De fato, se $P, Q$ são dois quaisquer deles, e se $f$ é uma das funções $z_{j}, \overline{z_{j}}, W_{k}, t_{l}$, então $\mathrm{d} f([P, Q])=$ $[P, Q] f=P(Q f)-Q(P f)=0$, pois $P(f)$ e $Q(f)$ são iguais a 0 ou 1. Como as diferenciais de $z_{j}, \overline{z_{j}}, W_{k}, t_{l}$ geram o fibrado cotangente, isso é o bastante para concluir que $[\mathrm{P}, \mathrm{Q}]=0$.

Os teoremas 1.53 e 1.54 acima fornecem representações locais canônicas para as integrais primeiras e os campos que geram uma estrutura localmente integrável, enfatizando o que ocorre com o conjunto característico $\mathrm{T}_{\mathrm{q}}^{0}$. Em geral, e inclusive nos próximos capítulos deste trabalho, não é necessário aplicá-los em toda essa generalidade, sendo suficiente enunciá-los como segue:

Corolário 1.55. Seja $\mathcal{V}$ localmente integrável de posto $n$. Seja $\mathrm{p} \in \Omega$, e denote por $\mathrm{m}$ o coposto $\mathrm{N}-\mathrm{n}$. Então existe um sistema de coordenadas local centrado em $\mathrm{p}$

$$
x_{1}, \ldots, x_{m}, t_{1}, \ldots, t_{n}
$$

e existem funções suaves $\phi_{1}, \ldots, \phi_{m}$, a valores reais, definidas em uma vizinhança de $\mathrm{p}$ e satisfazendo

$$
\phi_{k}(p)=0 \quad, \quad d_{x} \phi_{k}(p)=0 \quad, \quad k=1, \ldots, m
$$

tais que as diferenciais das funções suaves

$$
Z_{k}(x, t)=x_{k}+i \phi_{k}(x, t) \quad, \quad k=1, \ldots, m
$$

geram $\mathcal{V}^{\perp}$ em torno de $p$. 
Demonstração: $O$ que fizemos foi renomear as coordenadas

$$
x_{1}, \ldots, x_{v}, s_{1}, \ldots, s_{d}, y_{1}, \ldots, y_{v}, t_{1}, \ldots, t_{r}
$$

e as integrais primeiras $Z_{1}, \ldots, Z_{v}, W_{1}, \ldots, W_{d}$ dadas pelo teorema 1.53, chamando-as agora de $x_{1}, \ldots, x_{m}, t_{1}, \ldots, t_{n}$ e $Z_{1}, \ldots, Z_{m}$ (as novas variáveis $x$ correspondem às antigas $x$ e $s$, enquanto que as novas variáveis $t$ correspondem às antigas $y e t)$. Note que as primeiras $v$ funções $\phi_{k}$ obtidas aqui são as coordenadas $y_{1}, \ldots, y_{v}$ antigas.

Corolário 1.56. Seja $\mathcal{V}$ localmente integrável de posto $n$. Seja $\mathrm{p} \in \Omega$, e denote por $\mathrm{m}$ $o$ coposto $\mathrm{N}-\mathrm{n}$. Então existe um sistema de coordenadas local $\mathrm{x}_{1}, \ldots, \mathrm{x}_{\mathrm{m}}, \mathrm{t}_{1}, \ldots, \mathrm{t}_{\mathrm{n}}$ centrado em $p$ e existem campos $L_{1}, \ldots, L_{n}, M_{1}, \ldots, M_{m}$ que geram o fibrado tangente, comutam dois a dois e são dados no formato

$$
\mathrm{L}_{j}=\frac{\partial}{\partial \mathrm{t}_{j}}+\sum_{\rho=1}^{\mathrm{m}} \mathrm{a}_{\mathrm{j} \rho} \frac{\partial}{\partial x_{\rho}} \quad \mathrm{M}_{\mathrm{k}}=\sum_{\rho=1}^{\mathrm{m}} \mu_{\mathrm{k} \rho} \frac{\partial}{\partial x_{\rho}}
$$

onde $a_{j \rho}, \mu_{k \rho}$ são funções suaves, tais que $\mathcal{V}$ é gerada por $L_{1}, \ldots, L_{n}$.

Demonstração: $\operatorname{Sejam}(x, t)$ e $Z_{k}$ as coordenadas e as integrais primeiras dadas pelo corolário acima. Definimos as funções $\mu_{\mathrm{k} \rho}$ como os elementos da inversa do Jacobiano $\left(\partial_{x_{\rho}} Z_{k}\right)_{m \times m}$ em alguma vizinhança da origem, e então definimos os campos $M_{k}$ como no enunciado. Isso é suficiente para que tenhamos $M_{k} Z_{\rho}=\delta_{k \rho}$. Agora definimos

$$
\mathrm{L}_{\mathrm{j}}=\frac{\partial}{\partial \mathrm{t}_{\mathrm{j}}}-\mathrm{i} \sum_{\mathrm{k}=1}^{\mathrm{m}} \frac{\partial \phi_{\mathrm{k}}}{\partial \mathrm{t}_{\mathrm{j}}} \mathrm{M}_{\mathrm{k}}, \quad \mathrm{j}=1, \ldots, \mathrm{n}
$$

Cálculos diretos mostram que $\mathrm{L}_{\mathrm{j}} \mathrm{Z}_{\mathfrak{u}}=0, j=1, \ldots, n, z=1, \ldots, \mathrm{m}$, e portanto os $\mathrm{L}_{j}$ geram $v$ em torno de p. As outras afirmações seguem como na demonstração do teorema 1.54 .

Exemplo 1.58. Seja $\mathrm{U} \subseteq \mathbb{R}^{n}$ aberto. Uma estrutura tubular em $\mathbb{R}^{\mathrm{m}} \times \mathrm{U}$ é uma estrutura localmente integrável $\mathcal{V}$ sobre $\mathbb{R}^{\mathrm{m}} \times \mathrm{U}$ que admite integrais primeiras locais dadas na forma

$$
Z_{k}(x, t)=x_{k}+i \phi_{k}(t) \quad, \quad k=1, \ldots, m
$$

para alguma aplicação $\phi=\left(\phi_{1}, \ldots, \phi_{\mathrm{m}}\right)$ suave. A diferença nesse caso é que agora as funções $\phi$ dependem só de $t$. Nesse caso os campos $M_{k}$ do corolário acima são dados simplesmente por $\partial_{x_{\mathrm{k}}}$.

Proposição 1.59. Se $\Omega$ é uma variedade analítica e $\mathcal{V}$ é uma estrutura involutiva analitica (em particular, localmente integrável), então as funções $\phi_{\mathrm{k}}$ do corolário 1.55 podem ser tomadas analíticas, e satisfazendo

$$
\phi_{k}(x, 0)=0, k=1, \ldots, m
$$

para todo $(x, 0)$ suficientemente próximo a 0. 
Demonstração: Obtenha as funções $Z_{k}$ como naquele corolário, e defina $Z=\left(Z_{1}, \ldots, Z_{m}\right)$, que pelas demonstrações dos teoremas 1.52 e 1.53 é uma aplicação analítica. Estenda $Z$ holomorficamente a um aberto $\mathrm{U} \times \mathrm{V}$ de $\mathbb{C}^{\mathrm{m}} \times \mathbb{C}^{\mathrm{n}}$ contendo a origem. Considere a seguinte função em $\mathbb{C}^{\mathrm{m}} \times \mathbb{C}^{\mathrm{m}} \times \mathbb{C}^{\mathrm{n}}=\{(\zeta, x, t)\}$ a valores em $\mathbb{C}^{\mathrm{m}}$ :

$$
F(\zeta, x, t)=Z(x, t)-\zeta
$$

Temos $F(0,0,0)=0$, e o Jacobiano $\left(\partial_{x_{k}} F_{\rho}\right)_{m \times m}(\zeta, x, t)$, que é dado por

$$
\left(\frac{\partial Z_{\rho}}{\partial x_{k}}(x, t)\right)_{k, \rho=1, \ldots, m}
$$

é invertível em $(0,0,0)$. Pelo Teorema da Função Implícita (versão holomorfa), se diminuirmos $U$ e $V$ garantimos que existe uma função holomorfa $H(\zeta, t)=\left(H_{1}, \ldots, H_{m}\right)(\zeta, t)$ : $\mathrm{U} \times \mathrm{V} \rightarrow \mathbb{C}^{\mathrm{m}}$, definida em $\mathrm{U} \times \mathrm{V}$, tal que, para todo $x$ em uma certa vizinhança $\mathrm{W}$ da origem,

$$
\mathrm{H}(0,0)=0, \quad \mathrm{Z}(\mathrm{H}(\zeta, \mathrm{t}), \mathrm{t})=\zeta
$$

Em particular, se diminuirmos $W \times V$ para que $Z(x, t) \in U$ (por continuidade), teremos $Z(H(Z(x, t), t), t)=Z(x, t)$, e igualando as partes reais dos dois membros dessa equação obtemos

$$
H(Z(x, t), t)=x
$$

Defina então

$$
Z_{k}^{\sharp}(x, t)=H_{k}(Z(x, t), 0) \quad, \quad k=1, \ldots, m
$$

Observe que $Z_{k}^{\sharp}(x, 0)=H_{k}(Z(x, 0), 0)=x_{k}$, e que

$$
\begin{aligned}
d Z_{k}^{\sharp}= & \sum_{j=1}^{m} \frac{\partial Z_{k}^{\sharp}}{\partial x_{j}} d x_{j}+\sum_{l=1}^{n} \frac{\partial Z_{k}^{\sharp}}{\partial t_{l}} d t_{l} \\
= & \sum_{j=1}^{m}\left(\sum_{r=1}^{m} \frac{\partial H_{k}}{\partial \zeta_{r}}(Z(x, t), 0) \frac{\partial Z_{r}}{\partial x_{j}}(x, t)\right) d x_{j}+ \\
& +\sum_{l=1}^{n}\left(\sum_{r=1}^{m} \frac{\partial H_{k}}{\partial \zeta_{r}}(Z(x, t), 0) \frac{\partial Z_{r}}{\partial t_{l}}(x, t)\right) d t_{l} \\
= & \sum_{r=1}^{m} \frac{\partial H_{k}}{\partial \zeta_{r}}(Z(x, t), 0)\left(\sum_{j=1}^{m} \frac{\partial Z_{r}}{\partial x_{j}}(x, t) d x_{j}+\sum_{l=1}^{n} \frac{\partial Z_{r}}{\partial t_{l}}(x, t) d t_{l}\right) \\
= & \sum_{r=1}^{m} \frac{\partial H_{k}}{\partial \zeta_{r}}(Z(x, t), 0) d Z_{r}
\end{aligned}
$$

Mas, como a aplicação $x \mapsto \mathrm{H}(\mathrm{Z}(x, t), \mathrm{t})$ é a identidade, a regra da cadeia implica que

$$
\frac{\partial Z}{\partial x}(x, t)=\left(\frac{\partial H}{\partial \zeta}(Z(x, t), t)\right)^{-1} \Rightarrow \frac{\partial H}{\partial \zeta}(0,0)=I_{m \times m}
$$


Logo, (1.60) implica que os vetores cotangentes $\left(d Z_{k}^{\sharp}\right)_{(0,0)}$ e $\left(d Z_{k}\right)_{(0,0)}$ são o mesmo, para cada $\mathrm{k}=1, \ldots, \mathrm{m}$. Em particular, $\mathrm{d} Z_{1}^{\sharp}, \ldots, \mathrm{d} Z_{\mathrm{m}}^{\sharp}$ são l.i. na origem e em torno dela. Mais do que isso, essas formas geram $\mathcal{V}^{\perp}$ em torno da origem. De fato, basta provarmos que, se um campo $L$ satisfaz $L Z_{k}=0$, então também satisfaz $L Z_{k}^{\sharp}=0$, para $k=1, \ldots, m$, e isto segue imediatamente da regra da cadeia:

$$
\mathrm{LZ}_{\mathrm{k}}^{\sharp}=\sum_{\mathrm{r}=1}^{\mathrm{m}} \frac{\partial \mathrm{H}_{\mathrm{k}}}{\partial \zeta_{\mathrm{r}}}(\mathrm{Z}, 0) \mathrm{LZ}_{\mathrm{r}}=0
$$

Para cada $t$ fixo, o Jacobiano da aplicação $\chi \mapsto \Re Z^{\sharp}(x, t)$ é dado por

$$
\left(\mathfrak{R} \frac{\partial Z_{\rho}^{\sharp}(x, t)}{\partial x_{k}}\right)_{k, \rho}=\left(\Re \sum_{r=1}^{m} \frac{\partial H_{\rho}}{\partial \zeta_{r}}(Z(x, t), 0) \frac{\partial Z_{r}}{\partial x_{k}}(x, t)\right)_{k, \rho}
$$

que em $(x, t)=(0,0)$ vale

$$
\left(\mathfrak{R} \frac{\partial Z_{\rho}}{\partial x_{k}}(0,0)\right)_{k, \rho}=I_{m \times m}
$$

Logo, a aplicação $\sigma:(x, t) \mapsto(X, T)=\left(\Re Z^{\sharp}(x, t), t\right)$ é uma mudança de coordenadas perto da origem, e nessas novas coordenadas podemos escrever

$$
Z_{k}^{\sharp}(X, T)=X_{k}+i \phi_{k}^{\sharp}(X, T)
$$

para alguma função analítica $\phi_{k}^{\sharp}$. Finalmente, note que

$$
\sigma:(x, 0) \mapsto\left(\Re Z^{\sharp}(x, 0), 0\right)=(x, 0)
$$

e $\operatorname{assim} Z_{k}^{\sharp}(X, 0)=Z_{k}^{\sharp}(x, 0)=x_{k} \in \mathbb{R}$, isto é, $\phi_{k}^{\sharp}(X, 0)=0$ para todo $k$.

Corolário 1.61. Suponha que $\mathcal{V}$ é uma estrutura involutiva analítica, gerada em uma carta local $\mathrm{U}$ por campos $\mathrm{L}_{\mathrm{j}}$ no formato canônico do corolário 1.56 , isto é,

$$
\mathrm{L}_{\mathrm{j}}=\frac{\partial}{\partial \mathrm{t}_{j}}+\sum_{\rho=1}^{\mathrm{m}} \mathrm{a}_{\mathrm{j} \rho} \frac{\partial}{\partial x_{\rho}} \quad, \quad j=1, \ldots, n
$$

Suponha que as integrais primeiras $\mathrm{Z}_{1}, \ldots, \mathrm{Z}_{\mathrm{n}}$ estejam definidas em $\mathrm{U}$. Seja $\mathrm{h}=\mathrm{h}(\mathrm{x})$ analítica. Então o problema de Cauchy

$$
\left\{\begin{array}{l}
L_{j} u=0 \\
u(x, 0)=h(x)
\end{array}, \quad j=1, \ldots, n\right.
$$

admite uma única solução analítica em $\mathrm{U}$, dada por

$$
u(x, t)=h\left(Z_{1}(x, t), \ldots, Z_{n}(x, t)\right)
$$


Demonstração: Este problema é não-característico, dado que a superfície dos dados iniciais é $\{t=0\}$, enquanto que os campos $L_{j}$ não são paralelos a ela. Também, o dado inicial é analítico por hipótese, e os coeficientes dos campos são analíticos. De fato, de acordo com a equação (1.57), esses coeficientes são somas de parcelas na forma $\mu_{k \rho} \partial_{t_{j}} \phi_{k}$; as funções $\phi_{k}$ são analíticas pela proposição anterior, e as funções $\mu_{k \rho}$ também, porque foram obtidas como os elementos da inversa do Jacobiano $\left(\partial_{x_{\rho}} Z_{k}\right)$. Portanto, se existir uma solução analítica em alguma vizinhança da superfície $\{t=0\}$, ela será única, uma vez que todas suas derivadas estão determinadas nessa superfície. $E \mathrm{u}(x, t)$ dada no enunciado é de fato solução, pois

$$
\begin{gathered}
u(x, 0)=h\left(Z_{1}(x, 0), \ldots, Z_{m}(x, 0)\right)=h\left(x_{1}, \ldots, x_{m}\right)=h(x) \\
L_{j} u=\sum_{k=1}^{m} \frac{\partial h}{\partial x_{k}}\left(Z_{1}, \ldots, Z_{m}\right) L_{j} Z_{k}=0
\end{gathered}
$$

Teorema 1.62. Considere as mesmas condições do corolário acima, com $\mathrm{U}$ conexo por caminhos, e seja $f(x, t)=\left(f_{1}, \ldots, f_{n}\right)$ uma aplicação suave analítica satisfazendo as seguintes condições de compatibilidade: $\mathrm{L}_{\mathrm{j}} \mathrm{f}_{\mathrm{k}}=\mathrm{L}_{\mathrm{k}} \mathrm{f}_{\mathrm{j}}$ para todos $\mathrm{j}, \mathrm{k}$. Então a única solução analítica do problema de Cauchy em U

$$
\left\{\begin{array}{l}
L_{j} u=f_{j} \\
u(x, 0)=0
\end{array}, \quad j=1, \ldots, n\right.
$$

é dada pela integral de linha $\mathrm{u}(\mathrm{x}, \mathrm{t})=\int_{\gamma_{\mathrm{t}}} \overrightarrow{\mathrm{F}}_{\mathrm{x}, \mathrm{t}} \mathrm{d} \overrightarrow{\mathrm{r}}$ onde a curva $\gamma_{\mathrm{t}}$ é qualquer caminho retificável ligando 0 a $\mathrm{t}$, o campo $\overrightarrow{\mathrm{F}}_{x, \mathrm{t}}=\left(\mathrm{F}_{\mathrm{x}, \mathrm{t}}^{(1)}, \ldots, \mathrm{F}_{x, \mathrm{t}}^{(\mathrm{n})}\right)$ é dado por

$$
\overrightarrow{\mathrm{F}}_{x, \mathrm{t}}: \mathbb{R}^{n} \rightarrow \mathbb{R}^{n} \quad, \quad \overrightarrow{\mathrm{F}}_{x, t}(s)=\left(f_{1}\left(h_{s}(x, t), s\right), \ldots, f_{n}\left(h_{s}(x, t), s\right)\right)
$$

e, para cada s fixo, a aplicação $\mathrm{h}_{\mathrm{s}}=\left(\left(\mathrm{h}_{\mathrm{s}}\right)_{1}, \ldots,\left(\mathrm{h}_{\mathrm{s}}\right)_{\mathrm{m}}\right): \mathrm{U} \rightarrow \mathbb{R}^{\mathrm{m}}$ é a solução em $\mathrm{U}$ do problema

$$
\left\{\begin{array}{l}
L_{j} h_{s}=0 \\
h_{s}(x, s)=x
\end{array}, \quad j=1, \ldots, n\right.
$$

Este teorema pode ser considerado como uma generalização do chamado Princípio de Duhamel para equações de evolução (vide [J], seção 5.1c, p. 135). Observe que $h_{s}$ realmente existe e está definida em $U$ : cada coordenada $\left(h_{s}\right)_{k}$ pode ser obtida como no corolário 1.61.

Demonstração: A unicidade da solução analítica segue como acima. Também é claro que o dado inicial $u(x, 0)=0$ é satisfeito pela $u$ dada no enunciado; resta provarmos que $L_{j} u=f_{j}$ para cada $j$. Para simplificar a escrita, vamos assumir $m=1$, isto é, só existe 
uma variável $x$. A demonstração é exatamente a mesma quando $m>1$. Então existem funções analíticas $\lambda_{1}, \ldots, \lambda_{n}$ tais que os campos $L_{j}$ são escritos como

$$
L_{j}=\frac{\partial}{\partial t_{j}}+\lambda_{j}(x, t) \frac{\partial}{\partial x} \quad, \quad j=1, \ldots, n
$$

Denotemos a expressão $h_{s}(x, t)$ por $h(x, t, s)$. Logo o dado inicial no problema (1.63) implica que $h(x, s, s)=x$. Agora observe que

$$
\frac{\partial h}{\partial s_{j}}(x, t, s)=\lambda_{j}(h(x, t, s), s) \quad \text { para todos } x, t, s
$$

De fato, derivando-se $h(x, s, s)=\chi$ com relação a $s_{j}$ e usando o sistema (1.63), obtemos que

$$
\frac{\partial h}{\partial s_{j}}(x, s, s)=-\frac{\partial h}{\partial t_{j}}(x, s, s)=\lambda_{j}(x, s) \frac{\partial h}{\partial x}(x, s, s)
$$

Mas a equação $h(x, s, s)=x$ implica que $\lambda_{j}(x, s)=\lambda_{j}(h(x, s, s), s)$ e que $\partial_{x} h(x, s, s)=1$, e assim (1.65) se torna

$$
\frac{\partial h}{\partial s_{j}}(x, s, s)=\lambda_{j}(h(x, s, s), s)
$$

Por outro lado, tanto $\partial_{s_{j}} h(x, t, s)$ como $\lambda_{j}(h(x, t, s), s)$ são soluções do sistema $L_{k} w=0$, $\mathrm{k}=1, \ldots, \mathrm{n}$, a primeira porque $\partial_{s_{j}}$ comuta com $\mathrm{L}_{\mathrm{k}}$ e a segunda pela regra da cadeia. Juntando-se a isso a equação (1.66) acima, concluímos que ambas são soluções analíticas do problema de Cauchy

$$
\left\{\begin{array}{l}
\mathrm{L}_{k} w(x, t, s)=0 \\
w(x, s, s)=\partial_{s_{j}} h(x, s, s)
\end{array}, \quad k=1, \ldots, n\right.
$$

que tem unicidade de solução analítica, da mesma forma que o problema do corolário 1.61 (de fato, podemos considerá-lo como uma família de problemas nas variáveis $(x, t)$ parametrizada por s, e o raciocínio é o mesmo de antes). Isso prova a equação (1.64).

Com isso podemos concluir que, para cada $(x, t)$, o campo $\vec{F}_{x, t}$ é conservativo: para quaisquer $j, k$ dados,

$$
\begin{array}{r}
\frac{\partial F_{x, t}^{(k)}}{\partial s_{j}}(s)=\frac{\partial}{\partial s_{j}}\left(f_{k}(h(x, t, s), s)\right)=\frac{\partial f_{k}}{\partial x}(h(x, t, s), s) \frac{\partial h}{\partial s_{j}}(x, t, s)+\frac{\partial f_{k}}{\partial t_{j}}(h(x, t, s), s)= \\
=\frac{\partial f_{k}}{\partial x}(h(x, t, s), s) \lambda_{j}(h(x, t, s), s)+\frac{\partial f_{k}}{\partial t_{j}}(h(x, t, s), s)=\left(L_{j} f_{k}\right)(h(x, t, s), s)
\end{array}
$$

e analogamente

$$
\frac{\partial F_{x, t}^{(j)}}{\partial s_{k}}(s)=\left(L_{k} f_{j}\right)(h(x, t, s), s)
$$


o que nos diz, graças às condições de compatibilidade, que $\partial_{s_{j}} F_{x, t}^{(k)}=\partial_{s_{k}} F_{x, t}^{(j)}$, como queríamos. Assim existe um potencial para $\vec{F}_{x, t}$, isto é, uma função escalar $G_{x, t}=G_{x, t}(s)$ satisfazendo $\nabla G_{x, t}=\vec{F}_{x, t}$, e $u$ fica dada por

$$
u(x, t)=\int_{\gamma_{t}} \vec{F}_{x, t} d \vec{r}=G_{x, t}(t)-G_{x, t}(0)
$$

Calculamos $\mathrm{L}_{\mathrm{j}} \mathrm{u}$ pela regra da cadeia:

$$
L_{j} u(x, t)=\left(L_{j} G_{x, t}\right)(t)+\sum_{l=1}^{n} \frac{\partial G_{x, t}}{\partial s_{l}}(t)\left(L_{j} t_{l}\right)-\left(L_{j} G_{x, t}\right)(0)
$$

Agora afirmamos que $\left(L_{j} G_{x, t}\right)(t)=\left(L_{j} G_{x, t}\right)(0)$. De fato, observe que

$$
\begin{aligned}
\nabla\left(L_{j} G_{x, t}\right)(s) & =\left(\frac{\partial}{\partial s_{1}}\left(L_{j} G_{x, t}\right)(s), \ldots, \frac{\partial}{\partial s_{n}}\left(L_{j} G_{x, t}\right)(s)\right) \\
& =\left(L_{j} \frac{\partial}{\partial s_{1}} G_{x, t}(s), \ldots, L_{j} \frac{\partial}{\partial s_{n}} G_{x, t}(s)\right) \\
& =\left(L_{j}\left(f_{1}(h(x, t, s), s)\right), \ldots, L_{j}\left(f_{1}(h(x, t, s), s)\right)\right)=\overrightarrow{0}
\end{aligned}
$$

Logo $L_{j} G_{x, t}$ é constante em $U$. Com este fato, a equação (1.67) é simplificada:

$$
L_{j} u(x, t)=\sum_{l=1}^{n} \frac{\partial G_{x, t}}{\partial s_{l}}(t)\left(L_{j} t_{l}\right)=\frac{\partial G_{x, t}}{\partial s_{t}}(t)=f_{j}(h(x, t, t), s)=f_{j}(x, s)
$$

como queríamos provar.

\subsection{Teorema de Nirenberg}

Newlander e Nirenberg provaram em [NN] que toda estrutura involutiva complexa é localmente integrável. Não reproduziremos este resultado aqui, dada a extensão de sua demonstração e os detalhes envolvidos. Mas com base neste teorema é possível obter um corolário mais forte:

Corolário 1.68. (Nirenberg) Toda estrutura involutiva elíptica é localmente integrável.

Demonstração: Sejam $V$ uma estrutura involutiva elíptica de posto $n$ e coposto $m=$ $N-n$, e seja $p \in \Omega$ arbitrário. Então

$$
\mathcal{V}^{\perp} \oplus \overline{\mathcal{V}^{\perp}}=\bigcup_{q \in \Omega} \mathcal{V}_{p}^{\perp} \oplus \overline{\mathcal{V}_{p}^{\perp}}
$$


é um subfibrado cotangente de dimensão $2 \mathrm{~m}$. De fato, se $\omega_{1}, \ldots, \omega_{m}$ são formas que geram $\nu^{\perp}$ em uma vizinhança aberta de $p$, então $\omega_{1}, \ldots, \omega_{m}, \overline{\omega_{1}}, \ldots, \overline{\omega_{m}}$ geram $\mathcal{V}^{\perp} \oplus \overline{\nu^{\perp}}$ na mesma vizinhança. Note que em particular deduzimos que $2 \mathrm{~m} \leq \mathrm{N}$. Tomando o anulador, concluímos que

$$
\mathcal{V} \cap \overline{\mathcal{V}}=\bigcup_{\mathrm{q} \in \Omega} \mathcal{\nu}_{\mathrm{p}} \cap \overline{\mathcal{V}_{p}}
$$

é um subfibrado tangente de posto $r=N-2 m$.

Afirmamos que

$$
\nu \cap \mathrm{T} \Omega=\bigcup_{q \in \Omega} \nu_{p} \cap \mathrm{T}_{p} \Omega
$$

é um subfibrado de $\mathrm{T} \Omega$ de dimensão real $r$. Para provar isto, tome campos $\mathrm{L}_{1}, \ldots, \mathrm{L}_{\mathrm{r}} \in$ $X(\Omega)$ que geram $\mathcal{V} \cap \bar{V}$ sobre $\mathbb{C}$ em torno de $p$. Como $\mathcal{V} \cap \overline{\mathcal{V}}$ é essencialmente real, os campos reais $\mathfrak{R} L_{1}, \mathfrak{I} L_{1}, \ldots, \mathfrak{R} L_{r}, \mathfrak{I} L_{r}$ são seções desse fibrado e também o geram ali, sobre $\mathbb{R}$. Assim, existem $r$ deles que são l.i. em p e portanto também em uma vizinhança aberta de $p$ possivelmente menor. Resta vermos que o subfibrado gerado por eles é exatamente $\nu \cap \mathrm{T} \Omega$. Portanto o que devemos provar é que, se $v_{1}, \ldots, v_{\mathrm{r}} \in \mathrm{T}_{\mathrm{q}} \Omega$ satisfazem

$$
\operatorname{span}_{\mathbb{C}}\left\{v_{1}, \ldots, v_{\mathrm{r}}\right\}=\mathcal{V}_{\mathrm{q}} \cap \overline{\mathcal{V}_{\mathrm{q}}}
$$

então

$$
\operatorname{span}_{\mathbb{R}}\left\{v_{1}, \ldots, v_{\mathrm{r}}\right\}=\mathcal{V}_{\mathrm{q}} \cap \mathrm{T}_{\mathrm{q}} \Omega
$$

A inclusão " $\subseteq$ " em (1.69) está clara, já que cada $v_{i}$ pertence ao espaço vetorial real $V_{\mathrm{q}} \cap \mathrm{T}_{\mathrm{q}} \Omega$. Quanto à inclusão contrária, seja $v \in \mathcal{V}_{\mathrm{q}} \cap \mathrm{T}_{\mathrm{q}} \Omega$. Então $v=\bar{v} \Rightarrow v \in \mathcal{V}_{\mathrm{q}} \cap \overline{\mathcal{V}_{\mathrm{q}}} \Rightarrow v$ se escreve como

$$
v=\sum_{j=1}^{r} a_{i} v_{i} \quad, \quad a_{j} \in \mathbb{C}
$$

Como $v=\bar{v}$ e os $v_{i}$ são $l . i$. sobre $\mathbb{C}$, tomando o conjugado nessa igualdade concluímos que cada $a_{j}$ é real, e dessa forma $v \in \operatorname{span}_{\mathbb{R}}\left\{v_{1}, \ldots, v_{\mathrm{r}}\right\}$, como queríamos.

Agora apliquemos o Teorema de Frobenius ao subfibrado real $\mathcal{V} \cap T \Omega$ : podemos afirmar que existem coordenadas $x_{1}, \ldots, x_{2 m}, t_{1}, \ldots, t_{r}$ centradas em $p$ tais que $\nu \cap T \Omega$ é gerado por

$$
\frac{\partial}{\partial t_{1}}, \ldots, \frac{\partial}{\partial t_{r}}
$$

num aberto $U \ni p$. Complete o conjunto $\left\{\left(\partial_{t_{1}}\right)_{p}, \ldots,\left(\partial_{t_{r}}\right)_{p}\right\} \subseteq V_{p}$ com vetores tangentes $v_{1}, \ldots, v_{m}$ para obter uma base de $\nu_{p}$, e sejam $L_{1}, \ldots, L_{m} \in X(\Omega)$ tais que

$$
\left(L_{j}\right)_{p}=v_{j}, j=1, \ldots, m
$$

Observe que, por continuidade, temos que $\left\{\left(L_{1}\right)_{q}, \ldots,\left(L_{m}\right)_{q},\left(\partial_{t_{1}}\right)_{q}, \ldots,\left(\partial_{t_{r}}\right)_{q}\right\}$ é linearmente independente sobre $\mathbb{C}$ em algum aberto $\mathrm{V}$ com $p \in V \subseteq U$. Dessa forma, os campos

$$
\mathrm{L}_{1}, \ldots, \mathrm{L}_{\mathrm{m}}, \frac{\partial}{\partial \mathrm{t}_{1}}, \ldots, \frac{\partial}{\partial \mathrm{t}_{\mathrm{r}}}
$$


geram $\mathcal{V}$ sobre $V$, e cada $L_{k}$ é composto por derivações apenas nas variáveis $x$ :

$$
\mathrm{L}_{\mathrm{k}}=\sum_{\mathrm{l}=1}^{2 m} \mathrm{a}_{\mathrm{kl}}(x, \mathrm{t}) \frac{\partial}{\partial \mathrm{x}_{\mathrm{l}}}
$$

para funções $a_{k l} \in C^{\infty}(V)$. Mas como a matriz $A_{m \times 2 m}$ formada pelas funções $a_{k l}$ tem posto $m$, alguma matriz menor $\tilde{A}_{m \times m}$ é invertível em $p$ e também em alguma vizinhança aberta de p. Para simplificar a notação, suponha que $\tilde{A}$ é formada pelas $m$ primeiras colunas de A. Então, se considerarmos um vetor de $m$ campos $L^{\sharp}$ dados por $L^{\sharp}=\tilde{A}^{-1} L$, onde $L$ é o vetor dos campos $L_{k}$ originais, obtemos que

$$
\mathrm{L}_{\mathrm{k}}^{\sharp}=\frac{\partial}{\partial x_{k}}+\sum_{l=m+1}^{2 \mathrm{~m}} b_{k l}(x, t) \frac{\partial}{\partial x_{l}} \quad, \quad k=1, \ldots, \mathrm{m}
$$

para certas funções $b_{k l} \in C^{\infty}(V)$. Por isso, não há perda de generalidade em assumir que os campos $L_{k}$ sejam dados em $V$ pela expressão (1.70).

Note que, uma vez que $\left\{\mathrm{L}_{1}, \ldots, \mathrm{L}_{\mathrm{m}}, \partial_{\mathrm{t}_{1}}, \ldots, \partial_{\mathrm{t}_{\mathrm{r}}}\right\}$ gera $\mathcal{V}$, e por hipótese $\mathcal{V}+\overline{\mathcal{V}}=\mathbb{C} T \Omega$, conclui-se que

$$
\left\{\mathrm{L}_{1}, \ldots, \mathrm{L}_{\mathrm{m}}, \overline{\mathrm{L}_{1}}, \ldots, \overline{\mathrm{L}_{\mathrm{m}}}, \frac{\partial}{\partial \mathrm{t}_{1}}, \ldots, \frac{\partial}{\partial \mathrm{t}_{\mathrm{r}}}\right\} \text { gera } \mathbb{C} T \Omega .
$$

Mas a cardinalidade desse conjunto é menor ou igual a $2 m+r=N$. Portanto só pode ser $\mathrm{N}$, e se trata de um conjunto l.i. Em particular,

$$
\left\{\mathrm{L}_{1}, \ldots, \mathrm{L}_{\mathrm{m}}, \overline{\mathrm{L}_{1}}, \ldots, \overline{\mathrm{L}_{m}}\right\} \text { é linearmente independente sobre } \mathbb{C}
$$

Calculemos o seguinte comutador:

$$
\begin{aligned}
{\left[\frac{\partial}{\partial t_{j}}, L_{k}\right] } & =\frac{\partial}{\partial t_{j}}\left(\frac{\partial}{\partial x_{k}}+\sum_{l=m+1}^{2 m} b_{k l} \frac{\partial}{\partial x_{l}}\right)-\left(\frac{\partial}{\partial x_{k}}+\sum_{l=m+1}^{2 m} b_{k l} \frac{\partial}{\partial x_{l}}\right) \frac{\partial}{\partial t_{j}} \\
& =\frac{\partial^{2}}{\partial t_{j} \partial x_{k}}+\sum_{l=m+1}^{2 m}\left(\frac{\partial b_{k l}}{\partial t_{j}} \frac{\partial}{\partial x_{l}}+b_{k l} \frac{\partial^{2}}{\partial t_{j} \partial x_{l}}\right)-\frac{\partial^{2}}{\partial x_{k} \partial t_{j}}-\sum_{l=m+1}^{2 m} b_{k l} \frac{\partial^{2}}{\partial x_{l} \partial t_{j}} \\
& =\sum_{l=m+1}^{2 m} \frac{\partial b_{k l}}{\partial t_{j}}(x, t) \frac{\partial}{\partial x_{l}}
\end{aligned}
$$

Por $\mathcal{V}$ ser involutiva, devem existir funções $C_{k}, D_{j} \in C^{\infty}(V)$ tais que

$$
\sum_{l=m+1}^{2 m} \frac{\partial b_{k l}}{\partial t_{j}}(x, t) \frac{\partial}{\partial x_{l}}=\left[\frac{\partial}{\partial t_{j}}, L_{k}\right]=\sum_{k=1}^{m} C_{k}(x, t) L_{k}+\sum_{j=1}^{r} D_{j}(x, t) \frac{\partial}{\partial t_{j}}
$$

Se aplicarmos essa igualdade em $x_{k}(k=1, \ldots, m)$, vamos obter que $C_{k}$ é nula, e se aplicarmos em $t_{j}$, que $D_{j}$ é nula. Logo

$$
\sum_{l=m+1}^{2 m} \frac{\partial b_{k l}}{\partial t_{j}}(x, t) \frac{\partial}{\partial x_{l}}=0, \quad k=1, \ldots, m, j=1, \ldots, r
$$


Dessa forma as funções $b_{k l}$ não dependem de $t_{1}, \ldots, t_{r}$, e assim os campos $L_{k}$ dependem apenas de $x_{1}, \ldots, x_{2 m}$.

Deduzimos então que $L_{1}, \ldots, L_{m}$ geram uma estrutura involutiva $\mathcal{W}$ na subvariedade $V_{1}$ de $\Omega$ dada por $V \cap\{t=0\}$ (de dimensão $2 m$ ). Note que $\mathcal{W}$ é complexa. De fato, por um lado temos $\mathcal{V}_{\mathrm{q}} \oplus \overline{\mathcal{V}_{\mathrm{q}}}=\mathbb{C T}_{\mathrm{q}} \Omega$ para todo $\mathrm{q} \in \mathrm{V}$, mas por outro, os campos $\mathrm{L}_{\mathrm{k}}$ geram toda a parte de $\mathcal{V}$ correspondente às variáveis $x$, isto é, $\left\{\left(\mathrm{L}_{1}\right)_{\mathrm{q}}, \ldots,\left(\mathrm{L}_{\mathrm{m}}\right)_{\mathrm{q}}\right\}$ é base de $\mathbb{C T}_{\mathrm{q}} \mathrm{V}_{1}$ para todo $\mathrm{q} \in \mathrm{V}$. Aliando-se (1.71) a isto, temos $\mathcal{W}_{\mathrm{q}} \oplus \overline{\mathcal{W}_{\mathrm{q}}}=\mathbb{C T}_{\mathrm{q}} \mathrm{V}_{1}$, como queríamos. Observe também que o coposto dessa estrutura é $2 m-m=m$.

Pelo Teorema de Newlander-Nirenberg, existem $\mathrm{Z}_{1}, \ldots, \mathrm{Z}_{\mathrm{m}} \in \mathrm{C}^{\infty}\left(\mathrm{V}_{1}\right)$ tais que $\mathrm{L}_{\mathrm{k}} \mathrm{Z}_{\mathrm{l}}=$ 0 e $\left\{d Z_{1}, \ldots, d Z_{m}\right\}$ geram $\mathcal{W}^{\perp}$ em $V_{1}$. Por fim, a forma como trabalhamos com as coordenadas em $V$ permite estender essas funções àquele aberto, definindo-se

$$
\tilde{Z}_{l}(x, t)=Z_{l}(x), \quad l=1, \ldots, m
$$

Como $\partial_{t_{j}} \tilde{Z}_{l}=0$, esse é um sistema de integrais primeiras para $V$ em torno de p. Assim, $V$ é localmente integrável.

Observação 1.72. Vimos até aqui que estruturas involutivas elipticas, complexas, essencialmente reais e analíticas são localmente integráveis. Em [BCH], seção I.16, p. 38, está detalhado um exemplo de uma estrutura involutiva Cauchy-Riemann em $\mathbb{R}^{2 n+1}$ de posto $n$ que não é localmente integrável em nenhuma vizinhança da origem. Este exemplo está relacionado ao bem conhecido operador não-resolúvel de Hans-Lewy (vide [L]). Veja também [T3] para um estudo de estruturas não-localmente integráveis. 



\section{CAPÍTULO

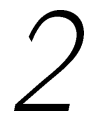 \\ Regularidade Analítica Local}

Este capítulo reproduz o trabalho [BT] de Baouendi e Trèves, um precursor da fórmula de aproximação que leva os nomes dos autores. Enquanto que os resultados presentes no artigo são provados de forma concisa, nosso objetivo nesta etapa foi escrevê-los com todos os detalhes necessários.

Aqui, $\Omega$ passará a denotar uma variedade analítica, e, para manter a notação original usada no artigo, vamos de encontro às convenções do capítulo anterior: $m+1$ denotará sua dimensão. Um ponto arbitrário $\omega_{0} \in \Omega$ está fixado, e em $\Omega$ está definida uma estrutura involutiva analítica $\mathbb{L}$ de coposto um, portanto de posto $\mathrm{m}$. Estaremos interessados em caracterizar a chamada hipoelipticidade analitica local em $\omega_{0}$ :

Definição 2.1. A estrutura $\mathbb{L}$ é dita analítica hipoelíptica $(\boldsymbol{A} \boldsymbol{H})$ em $\omega_{0}$ se o seguinte ocorre: para toda vizinhança aberta $\mathrm{U}$ de $\omega_{0}$ e toda distribuição $u \in \mathcal{D}^{\prime}(\mathrm{U})$, se para toda seção $M$ de $\mathbb{L}$ sobre $\mathrm{U}$ existir uma vizinhança aberta $\mathrm{V}_{M}$ de $\omega_{0}$ satisfazendo $\mathrm{Mu} \in \mathrm{C}^{\omega}\left(\mathrm{V}_{\mathrm{M}}\right)$, então existe uma vizinhança aberta $\mathrm{V}$ de $\omega_{0}$ satisfazendo $\mathrm{u} \in \mathrm{C}^{\omega}(\mathrm{V})$.

Caso $\mathbb{L}$ seja gerada em um aberto $\Omega^{\prime}$ por $m$ campos $L_{1}, \ldots, L_{m}$, esta definição toma a seguinte forma: para toda distribuição $u \in \mathcal{D}^{\prime}\left(\Omega^{\prime}\right)$, se existir uma vizinhança aberta $U$ de $\omega_{0}$ satisfazendo $L_{j} u \in C^{\omega}(U)$ para cada $j=1, \ldots, m$, então existe uma vizinhança aberta $V$ de $\omega_{0}$ satisfazendo $u \in C^{\omega}(V)$.

Um conceito que estará relacionado ao anterior é o seguinte:

Definição 2.2. Uma aplicação $\mathrm{f}: \mathrm{X} \rightarrow \mathrm{Y}$ entre dois espaços topológicos é dita aberta em $x \in \mathrm{X}$ se existe uma vizinhança aberta $\mathrm{U} \ni \mathrm{x}$ tal que, para qualquer aberto $\tilde{\mathrm{U}} \subseteq \mathrm{U}$ contendo $x$, a imagem $f(\tilde{u})$ é um aberto contendo $f(x)$. 


\subsection{Princípio da constância local}

Por se tratar de um estudo local, podemos nos valer de todas as formas canônicas locais deduzidas no capítulo anterior. Inicialmente, podemos supor que $\omega_{0}=0$ e que $\Omega$ é um aberto de $\mathbb{R}^{\mathrm{m}+1}$, e então, diminuindo $\Omega$ se necessário, existem coordenadas $t_{1}, \ldots, t_{\mathrm{m}}, x$ de $\mathbb{R}^{m+1}$ tais que $\mathbb{L}$ é gerada em $\Omega$ por campos da forma

$$
\mathrm{L}_{j}=\frac{\partial}{\partial \mathrm{t}_{j}}+\lambda_{j}(t, x) \frac{\partial}{\partial x} \quad, \quad j=1, \ldots, \mathrm{m}
$$

onde $\lambda_{j}$ são funções analíticas em $\Omega$ a valores complexos. Ainda, por se tratar de uma estrutura analítica involutiva, sabemos que $\mathbb{L}$ é localmente integrável; logo também podemos supor que uma integral primeira $z(t, x)$ está definida em $\Omega$, e tem a forma

$$
z(t, x)=x+i \phi(t, x)
$$

para alguma $\phi$ analítica a valores reais, que satisfaz $\phi(0, x)=0$ para todo $x$ (tal que $(0, x) \in \Omega)$.

Podemos escrever cada $\lambda_{j}$ em função de $\phi$ :

$$
0=L_{j} z=\frac{\partial z}{\partial t_{j}}+\lambda_{j} \frac{\partial z}{\partial x}=i \phi_{t_{j}}+\lambda_{j}\left(1+i \phi_{x}\right) \Rightarrow \lambda_{j}(t, x)=\frac{-i \phi_{t_{j}}(t, x)}{1+i \phi_{x}(t, x)}
$$

Em particular, nos pontos onde $d_{t} \phi=0$ (isto é, todas as derivadas de $\phi$ com relação às variáveis $t$ se anulam), as funções $\lambda_{j}$ são todas nulas, e os campos $L_{j}$ se reduzem a $\partial_{t_{j}}$. Esta observação nos será útil futuramente.

Lema 2.5. Os campos $\mathrm{L}_{j}$ comutam.

Demonstração: O corolário 1.56 pode ser invocado para provar este lema, mas daremos aqui a prova que aparece em [BT]. Como $\mathbb{L}$ é involutiva, para cada $j, k=1, \ldots, m$ existem funções $A_{j k}^{1}, \ldots, A_{j k}^{m} \in C^{\omega}(\Omega)$ tais que

$$
\left[L_{j}, L_{k}\right]=\sum_{v=1}^{m} A_{j k}^{v}(t, x) L_{v}=\sum_{v=1}^{m} A_{j k}^{v}(t, x) \frac{\partial}{\partial t_{v}}+\left(\sum_{v=1}^{m} A_{j k}^{v} \lambda_{v}(t, x)\right) \frac{\partial}{\partial x}
$$

Calculemos o colchete de Lie utilizando a expressão (2.3):

$$
\begin{aligned}
& {\left[\mathrm{L}_{j}, \mathrm{~L}_{k}\right] }=\left(\frac{\partial}{\partial t_{j}}+\lambda_{j} \frac{\partial}{\partial x}\right)\left(\frac{\partial}{\partial t_{k}}+\lambda_{k} \frac{\partial}{\partial x}\right)-\left(\frac{\partial}{\partial t_{k}}+\lambda_{k} \frac{\partial}{\partial x}\right)\left(\frac{\partial}{\partial t_{j}}+\lambda_{j} \frac{\partial}{\partial x}\right) \\
&= \frac{\partial^{2}}{\partial t_{j} \partial t_{k}}+\frac{\partial \lambda_{k}}{\partial t_{j}} \frac{\partial}{\partial x}+\lambda_{k} \frac{\partial^{2}}{\partial t_{j} \partial x}+\lambda_{j} \frac{\partial^{2}}{\partial x \partial t_{k}}+\lambda_{j} \lambda_{k} \frac{\partial^{2}}{\partial x^{2}}- \\
& \quad-\frac{\partial^{2}}{\partial t_{k} \partial t_{j}}-\frac{\partial \lambda_{j}}{\partial t_{k}} \frac{\partial}{\partial x}-\lambda_{j} \frac{\partial^{2}}{\partial t_{k} \partial x}-\lambda_{k} \frac{\partial^{2}}{\partial x \partial t_{j}}-\lambda_{k} \lambda_{j} \frac{\partial^{2}}{\partial x^{2}} \\
&=\left(\frac{\partial \lambda_{k}}{\partial t_{j}}+\lambda_{j} \frac{\partial \lambda_{k}}{\partial x}-\frac{\partial \lambda_{j}}{\partial t_{k}}-\lambda_{k} \frac{\partial \lambda_{j}}{\partial x}\right) \frac{\partial}{\partial x} \\
&=\left(L_{j} \lambda_{k}-L_{k} \lambda_{j}\right) \frac{\partial}{\partial x}
\end{aligned}
$$


Assim, $\left[L_{j}, L_{k}\right]$ apenas possui derivação em $x$. Mas em (2.6) havíamos visto que o coeficiente de $\partial_{t_{v}}$ em $\left[L_{j}, L_{k}\right]$ é $A_{j k}^{v}$. Dessa forma, cada função $A_{j k}^{v}$ é nula, e substituindo isto em (2.6) concluímos que $\left[\mathrm{L}_{\mathrm{j}}, \mathrm{L}_{\mathrm{k}}\right]=0$.

Observando que $z_{x}$ nunca se anula, introduza em $\Omega$ o campo

$$
\mathrm{L}_{0}=\frac{1}{z_{x}(\mathrm{t}, x)} \frac{\partial}{\partial x}
$$

Temos então $\mathrm{L}_{0} z=1$. Considerar $\mathrm{L}_{0}$ é um passo natural, assim como foi feito em (1.56), com a família de campos $M_{k}$. Como agora o coposto é 1 , só existe um $M_{k}$, que é justamente o campo $\mathrm{L}_{0}$.

Lema 2.7. O campo $\mathrm{L}_{0}$ comuta com os campos $\mathrm{L}_{\mathrm{j}}$.

Demonstração: Novamente, poderíamos recorrer ao corolário 1.56, mas segue abaixo a demonstração dos autores em [BT]. Para facilitar a notação, seja $a(t, x)=z_{\chi}(t, x)^{-1}$. Calculemos:

$$
\begin{aligned}
{\left[\mathrm{L}_{j}, \mathrm{~L}_{0}\right] } & =\left(\frac{\partial}{\partial t_{j}}+\lambda_{j} \frac{\partial}{\partial x}\right)\left(a \frac{\partial}{\partial x}\right)-\left(a \frac{\partial}{\partial x}\right)\left(\frac{\partial}{\partial t_{j}}+\lambda_{j} \frac{\partial}{\partial x}\right) \\
& =\frac{\partial a}{\partial t_{j}} \frac{\partial}{\partial x}+a \frac{\partial^{2}}{\partial t_{j} \partial x}+\lambda_{j} \frac{\partial a}{\partial x} \frac{\partial}{\partial x}+\lambda_{j} a \frac{\partial^{2}}{\partial x^{2}}-a \frac{\partial^{2}}{\partial x \partial t_{j}}-a \frac{\partial \lambda_{j}}{\partial x} \frac{\partial}{\partial x}-a \lambda_{j} \frac{\partial^{2}}{\partial x^{2}} \\
& =\left(\frac{\partial a}{\partial t_{j}}+\lambda_{j} \frac{\partial a}{\partial x}-a \frac{\partial \lambda_{j}}{\partial x}\right) \frac{\partial}{\partial x}= \\
& =\left(L_{j} a-a \frac{\partial \lambda_{j}}{\partial x}\right) \frac{\partial}{\partial x}
\end{aligned}
$$

Então o que devemos provar é que $L_{j} a-a \frac{\partial \lambda_{j}}{\partial x}=0$. Derivando-se a equação $L_{j} z=0$ com respeito a $x$, temos

$$
0=\frac{\partial}{\partial x}\left(\frac{\partial z}{\partial t_{j}}+\lambda_{j} \frac{\partial z}{\partial x}\right)=\frac{\partial z_{x}}{\partial t_{j}}+z_{x} \frac{\partial \lambda_{j}}{\partial x}+\lambda_{j} \frac{\partial z_{x}}{\partial x}=L_{j}\left(a^{-1}\right)+\left(a^{-1}\right) \frac{\partial \lambda_{j}}{\partial x}
$$

Devido ao teorema 1.8 , temos $L_{j}\left(a^{-1}\right)=-\left(L_{j} a\right) / a^{2}$ (note que a função $\zeta \mapsto 1 / \zeta$ é holomorfa na imagem de $a)$, e assim a equação (2.8) se torna

$$
0=-\frac{L_{j} a}{a^{2}}+a^{-1} \frac{\partial \lambda_{j}}{\partial x} \Rightarrow 0=-L_{j} a+a \frac{\partial \lambda_{j}}{\partial x}
$$

como queríamos.

Fixe $r>0$ e um intervalo aberto $\mathrm{J} \ni 0$ da reta tais que $\mathcal{B}_{\mathrm{r}} \times \mathrm{J} \subset \subset \Omega$, onde $\mathcal{B}_{\mathrm{r}}$ denota a bola de centro 0 e raio $r$ em $\mathbb{R}^{m}=\left\{\left(t_{1}, \ldots, t_{m}\right)\right\}$. Definimos

$$
\mathcal{N}_{0}=\left\{x \in \overline{\mathrm{J}} ; \phi(\cdot, x) \text { é a função nula em } \overline{\mathcal{B}_{\mathrm{r}}}\right\}
$$


Suponha inicialmente que $\mathcal{N}_{0}$ tenha um ponto de acumulação. Dessa forma, para cada $t \in \overline{\mathcal{B}_{\mathrm{r}}}$ fixado, a função $\phi(t, \cdot)$, que é analítica de uma variável real, teria zeros acumulandose em $\overline{\mathrm{J}}$, o que implicaria que $\phi(t, x)=0$ para todo $(t, x) \in \overline{\mathcal{B}_{\mathrm{r}}} \times \overline{\mathrm{J}}$. Em particular, também $d_{t} \phi$ seria identicamente nula, e, conforme observamos acima, isto implicaria que $L_{j}=\partial_{t_{j}}$ em $\overline{\mathcal{B}_{r}} \times \bar{J}$. Então o estudo da estrutura $\mathbb{L}$ se reduziria ao estudo da derivada exterior $\mathrm{d}_{\mathrm{t}}$, já amplamente abordado na literatura (os resultados a serem provados aqui valem trivialmente nesse caso). Por esse motivo, trabalharemos sob a hipótese adicional de que $\mathcal{N}_{0}$ não acumula em $\bar{J}$. Por compacidade deste último, temos que $\mathcal{N}_{0}$ é finito.

Listemos algumas propriedades do domínio e da imagem da função $z$. Veja também a figura 2.1 abaixo. Para cada $x \in \bar{J}$ fixado, a imagem de $\overline{\mathcal{B}_{\mathrm{r}}} \times\{x\}$ por $z$ está contida na reta vertical $\{\zeta \in \mathbb{C} ; \mathfrak{R} \zeta=\chi\}$. Mais ainda, como $\overline{\mathcal{B}_{\mathrm{r}}} \times\{x\}$ é compacto e conexo e $z$ é contínua, podemos afirmar que existe um intervalo $\mathrm{I}(\mathrm{x})=\left[\alpha_{x}, \beta_{x}\right]$ da reta, para certos $\alpha_{x} \leq \beta_{x}$, tal que

$$
z\left(\overline{\mathcal{B}_{\mathrm{r}}} \times\{x\}\right)=x+i \mathrm{I}(x)=\{\zeta \in \mathbb{C} ; \mathfrak{R} \zeta=x, \mathfrak{I} \zeta \in \mathrm{I}(x)\}
$$

De acordo com a definição de $\mathcal{N}_{0}$, temos $\alpha_{x}=\beta_{x}=0$ se e somente se $x \in \mathcal{N}_{0}$. Note ainda que $0 \in \mathrm{I}(\mathrm{x})$, uma vez que $\mathfrak{I} z=\phi$ e $\phi(0, x)=0$ para todo $x$. Mas pode ocorrer de 0 estar no interior do intervalo $\mathrm{I}(\mathrm{x})$ ou na fronteira. Mais precisamente, para o ponto que nos interessa:

Proposição 2.9. O ponto 0 está no interior de $\mathrm{I}(0)$ se e somente se z é aberta em 0 .

Demonstração: Se $0 \in \partial \mathrm{I}(0)$, então, para qualquer aberto $U \ni 0 \in \mathbb{R}^{\mathrm{m}+1}$, se definirmos $\tilde{\mathrm{U}}=\mathrm{U} \cap\left(\mathcal{B}_{\mathrm{r}}\right) \times \mathrm{J}$ teremos que $z(\tilde{\mathrm{U}})$ não é uma vizinhança aberta de $0 \in \mathbb{C}$, porque se o fosse existiriam $\left(t^{(1)}, 0\right),\left(t^{(2)}, 0\right) \in \tilde{U}$ tais que

$$
\Im z\left(t^{(1)}, 0\right)>0, \quad \Im z\left(t^{(2)}, 0\right)<0
$$

o que implicaria $0 \in \mathrm{I}(0)^{\circ}$. Reciprocamente, se $0 \in \mathrm{I}(0)^{\circ}$ então por continuidade existe $\eta>0$ tal que $0 \in \mathrm{I}(x)^{\circ}$ para todo $x \operatorname{com}|x|<\eta$. Seja o aberto

$$
\begin{aligned}
\mathrm{U}=\left(\mathcal{B}_{\mathrm{r}} \times J\right) \cap z^{-1}\{\zeta \in \mathbb{C} ; \mathfrak{R} \zeta \in(-\eta, \eta), \Im \zeta \in \mathrm{I}(\mathfrak{R} \zeta)\}= & \\
& =\left\{(\mathrm{t}, \mathrm{x}) \in \mathbb{R}^{\mathrm{m}+1} ; x \in(-\eta, \eta), \mathrm{t} \in \mathcal{B}_{\mathrm{r}}\right\}
\end{aligned}
$$

Como $z(t, x)=x+i \phi(t, x)$, é claro que $z(U)$ é aberto em torno de $0 \in \mathbb{C}$ para qualquer $\mathrm{U} \subseteq \tilde{\mathrm{U}}$ aberto em torno de $0 \in \mathbb{R}^{\mathrm{m}+1}$.

Também é útil observar que as fibras de $z$ em $\overline{\mathcal{B}_{\mathrm{r}}} \times \overline{\mathrm{J}}$, isto é, seus conjuntos de nível, são fechadas e estão contidas em conjuntos da forma $H_{x}=\left\{(x, t) ; t \in \overline{\mathcal{B}_{r}}\right\}$, para $x \in \overline{\mathrm{J}}$, já que $\mathfrak{R z}=x$ (chamaremos esses conjuntos de hiperplanos). Ainda, uma fibra contida em um hiperplano $H_{x}$ coincide com $H_{x}$ se e somente se $x \in \mathcal{N}_{0}$, porque $\Im z=\phi$ e $\mathcal{N}_{0}$ é composto precisamente dos pontos $x$ tais que $\phi$ é constante em $\mathrm{H}_{x}$. 
Denotaremos por $\mathrm{W} \subseteq \mathbb{C}$ a imagem de $\overline{\mathcal{B}_{r}} \times \overline{\mathrm{J}}$ por $z$. Logo,

$$
W=\bigcup_{x \in \bar{J}}(x+i I(x))
$$

e devido ao fato de $\mathcal{N}_{0}$ ser finito, o interior $W^{\circ}$ de $W$ em $\mathbb{C}$ é não-vazio.

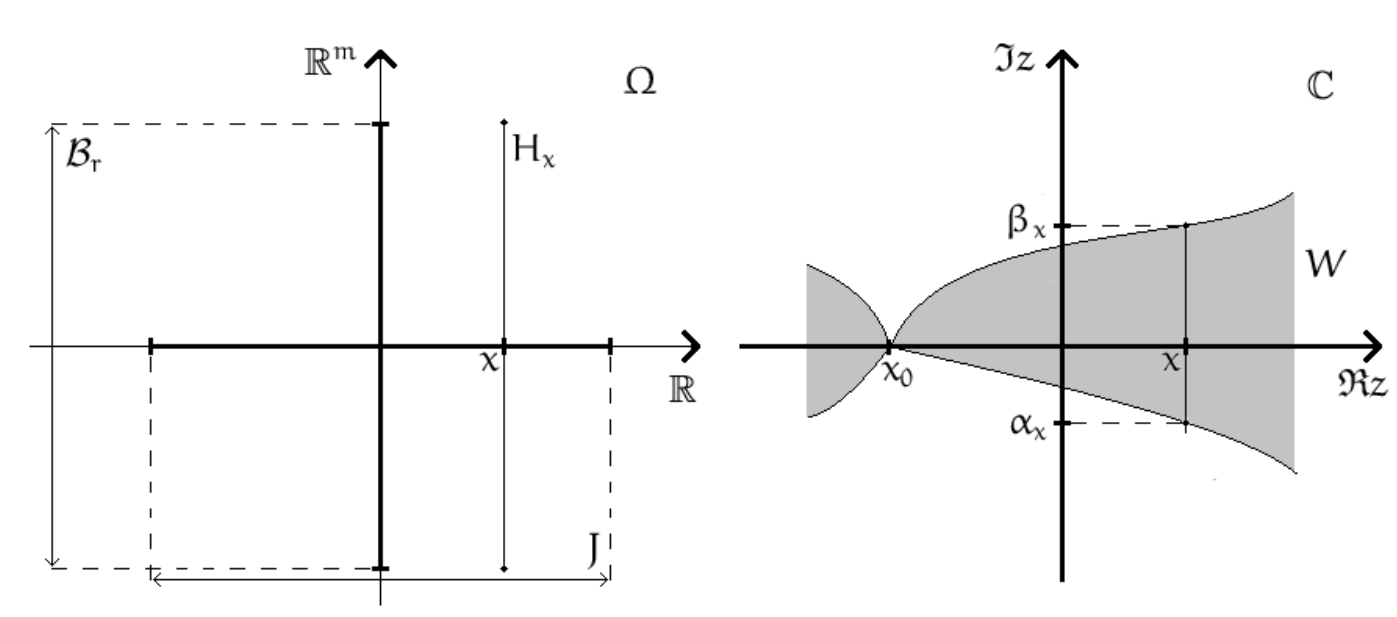

Figura 2.1: Dominio e imagem da função z. Neste exemplo, $x_{0}$ é o único ponto em $\mathcal{N}_{0}$, portanto o interior de $\mathrm{W}$ tem duas componentes conexas.

Lema 2.10. Sejam $\mathrm{r}, \mathrm{s}>0$. Se $\mathrm{f}:[-\mathrm{r}, \mathrm{r}] \times[-\mathrm{s}, \mathrm{s}] \rightarrow \mathbb{C}$ é analítica, isto é, $\mathrm{f}$ é analítica em um aberto da forma $(-\mathrm{r}-\delta, \mathrm{r}+\delta) \times(-\mathrm{s}-\delta, \mathrm{s}+\delta)$, com $\delta>0$, e se a função $\mathrm{f}(\cdot, 0)$ tem apenas finitos zeros em $[-r, r]$, então existem $M \geq 0$ e $\varepsilon>0$ tais que, para cada $x \in(0, \varepsilon]$, a função $f(\cdot, x)$ tem exatamente $M$ zeros em $[-r, r]$.

Demonstração: Sem perda de generalidade, podemos assumir, diminuindo $s$, que, para cada $x \in[-s, s]$, a função $f(\cdot, x)$ tem apenas finitos zeros em $[-r, r]$. Isto se deve ao mesmo argumento que usamos acima quando concluímos que $\mathcal{N}_{0}$ é finito. Da mesma forma, podemos assumir que $f$ não tem zeros isolados em $[-r, r] \times([-s, s] \backslash\{0\})$.

Note inicialmente que só há finitos zeros isolados e finitos zeros não-isolados de $f$ na forma $(t, 0)$. Seja

$$
Z=\{t \in[-r, r] ;(t, 0) \text { é zero não-isolado de } f\}
$$

O conjunto de zeros de $f$ em $[-r, r] \times[-s, s]$ é dado pelos seus zeros isolados em $\{x=0\}$ mais finitas curvas analíticas, e podemos diminuir $s$ de forma que cada uma passe por pelo menos um dos zeros não-isolados em $\{x=0\}$. Essas curvas podem sofrer bifurcações, mas diminuindo $s$ podemos assumir que as únicas bifurcações dentro de $[-r, r] \times[0, s)$ ocorrem em pontos de $\{x=0\}$. Veja a figura 2.2 abaixo.

Dessa forma, se $(t, 0)$ é um zero não-isolado de $f$, existem $N_{t}$ curvas analíticas $\gamma_{j}^{t}=$ $\left(\alpha_{j}^{\mathrm{t}}(\theta), \beta_{j}^{\mathrm{t}}(\theta)\right), j=1, \ldots, N_{t}, \theta \in\left[0, \theta_{0}\right), \beta_{j}^{\mathrm{t}}(\theta)>0$ para $\theta>0$ próximo a 0 , todas partindo 
de $(t, 0)$, isto é, $\alpha_{j}^{t}(0)=t$ e $\beta_{j}^{t}(0)=0$, e existe $r_{t}>0$ tal que o conjunto dos zeros de $f$ na semibola aberta $\mathcal{B}^{\mathrm{t}}=\mathcal{B}\left((t, 0), r_{t}\right) \cap\{x>0\}$ é precisamente

$$
\bigcup_{j=1}^{N_{t}} \gamma_{j}^{t} \cap \mathcal{B}^{t}
$$

Diminuindo $r_{t}$, podemos assumir que as $\gamma_{j}^{t}$ dentro de $\mathcal{B}^{t}$ não se interceptam. Mas, para cada $j$, a cardinalidade de $\left\{\theta \in\left[0, \theta_{0}\right) ;\left(\beta_{j}^{t}\right)^{\prime}(\theta)=0\right\}$ é finita, porque $f(\cdot, x)$ tem apenas finitos zeros em $[-r, r]$ para todo $x$. Em particular, podemos diminuir $s$ e assumir que $\left(\beta_{j}^{t}\right)^{\prime}(\theta) \neq 0$ para todos $j, \theta$. Então todas as $\beta_{j}^{t}$ são estritamente crescentes, o que implica que existe $0<R_{t}<r_{t}$ tal que a quantidade de zeros de $f$ em cada fatia $\mathcal{B}^{t} \cap\left\{x=x_{0}\right\}$, $0<x_{0}<R_{t}$, é exatamente $N_{t}$.

Seja $\varepsilon>0$ menor que todos os $R_{t}$, variando $t$ sobre $Z$. Então qualquer hiperplano $\left\{(t, x) ; t \in[-r, r], x=x_{0}\right\}$ com $0<x_{0} \leq \varepsilon$ cruza exatamente $\sum_{t \in Z} N_{t}$ zeros de $f$ dentro de $\cup_{t \in Z} \overline{\mathcal{B}^{t}}$, e não cruza nenhum zero fora dessa reunião: caso isso acontecesse, esse zero pertenceria a alguma curva $\gamma_{j}^{\mathrm{t}}$, e pelo Teorema de Rolle existiria $\theta>0$ tal que $\left(\beta_{j}^{\mathrm{t}}\right)^{\prime}(\theta)=0$, absurdo. O lema está provado.

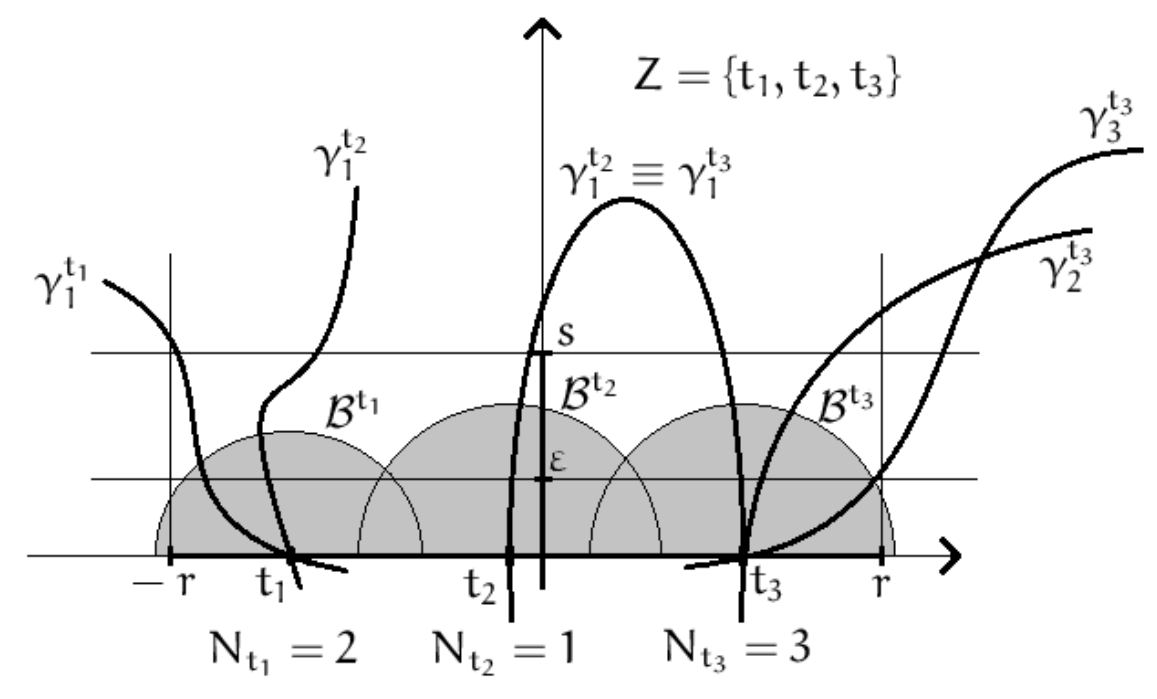

Figura 2.2: Demonstração do lema 2.10, exemplificando situações que podem ocorrer. Note que existem duas curvas $\gamma_{j}^{\mathrm{t}}$ que coincidem, mas diminuindo-se s podemos considerar separadamente dois pedaços, cada um partindo de um zero de $f$ em $\{x=0\}$. Também, algumas curvas $\gamma_{j}^{\mathrm{t}}$ se interceptam ou se tornam horizontais, mas apenas para $x>s$.

Note que, devido às bifurcações das curvas $\gamma_{j}^{t}$ que podem ocorrer em $\{x=0\}$, em geral não existe $\varepsilon>0$ tal que $f(\cdot, x)$ tenha a mesma quantidade de zeros em $[-r, r]$ para todo $x \in[-\varepsilon, \varepsilon]$. O que podemos fazer é aplicar o mesmo lema ao semiplano $x<0$, obtendo um outro número $M^{\prime}$ de zeros de $f(\cdot, x)$ em cada hiperplano $x=x_{0},-\varepsilon \leq x_{0}<0$. 
Lema 2.11. Nas condições do lema anterior, se $f$ é a valores reais, existem inteiros não-negativos $M_{1}, M_{2}, \ldots$, quase todos nulos, e $\varepsilon>0$ tais que, para cada $x \in(0, \varepsilon] e$ cada $\mathrm{j}=1,2, \ldots$, a função $\mathrm{f}(\cdot, \mathrm{x})$ tem exatamente $\mathrm{M}_{\mathrm{j}}$ zeros em $[-\mathrm{r}, \mathrm{r}]$ com multiplicidade j.

Demonstração: Como $f$ é a valores reais, os zeros de $f(\cdot, x)$ com multiplicidade pelo menos $j$, para cada $x \in[-s, s]$ fixo, são exatamente os zeros de

$$
g_{j}(t, x)=(f(t, x))^{2}+\left(\frac{\partial f}{\partial x}(t, x)\right)^{2}+\left(\frac{\partial^{2} f}{\partial x^{2}}(t, x)\right)^{2}+\cdots+\left(\frac{\partial^{j-1} f}{\partial x^{j-1}}(t, x)\right)^{2}
$$

Ainda, os zeros de $g_{j}$ são também zeros de $g_{k}$ para $k<j$. Se $j_{0}$ é a maior multiplicidade que ocorre com relação à variável $x$ dos zeros de $f$ em $[-r, r] \times[-s, s]$, então basta aplicarmos o lema anterior a cada função analítica $g_{1}, \ldots, g_{j_{0}}$ e tomarmos o menor dos $\varepsilon>0$ obtidos.

Lema 2.12. Sejam D $\subseteq \mathbb{C}$ um dominio e $\delta \subseteq \mathrm{D}$ uma reunião finita de conjuntos analíticos próprios, isto é, de conjuntos de zeros de funções holomorfas não identicamente nulas. Se $\mathrm{f}: \mathrm{D} \rightarrow \mathbb{C}$ é continua em $\mathrm{D}$ e holomorfa em $\mathrm{D} \backslash \delta$, então $\mathrm{f} e ́$ holomorfa em D.

A ideia aqui é que o conjunto $\delta$ tem medida de Lebesgue nula e é formado por uma quantidade finita de curvas retificáveis. Estas hipóteses são suficientes para concluirmos que f é holomorfa em D, de acordo com o teorema 5.3, cap. 6, p. 197 em [S].

O próximo teorema é um dos dois principais resultados no artigo [BT], e justifica a constância local mencionada em seu título.

Teorema 2.13. (Baouendi-Trèves) Para todo $\omega_{0} \in \Omega$, existe um aberto $\Omega^{\prime} \ni \omega_{0}$ tal que, para qualquer integral primeira $z \in \mathrm{C}^{\omega}\left(\Omega^{\prime}\right)$ e qualquer $u \in \mathrm{C}^{0}\left(\overline{\Omega^{\prime}}\right)$ solução de $\mathbb{L}$ em $\Omega^{\prime}$ (em sentido distribucional), existe

$$
\tilde{u}: W^{\prime} \rightarrow \mathbb{C} \quad, \quad \tilde{u} \in C^{0}\left(W^{\prime}\right) \cap \mathcal{O}\left(W^{\prime \circ}\right)
$$

(onde $\mathrm{W}^{\prime}=z\left(\overline{\Omega^{\prime}}\right)$ ) de forma que vale a decomposição

$$
u=\tilde{u} \circ z \quad e m \overline{\Omega^{\prime}}
$$

Em particular, u é constante ao longo das fibras de $z$ em $\overline{\Omega^{\prime}}$.

Demonstração: Fazendo uso das coordenadas locais e da forma canônica dos campos e da integral primeira observados acima, o que provaremos é que existe um intervalo aberto $\mathrm{J}^{\prime}=(-\varepsilon, \varepsilon) \subseteq \mathrm{J}$ em torno da origem tal que, para toda $u \in \mathrm{C}^{0}\left(\overline{\mathcal{B}_{\mathrm{r}}} \times \overline{\mathrm{J}^{\prime}}\right)$ solução de $\mathbb{L}$ em $\mathcal{B}_{\mathrm{r}} \times \mathrm{J}^{\prime}$ (em sentido distribucional), existe

$$
\tilde{u}: W^{\prime} \rightarrow \mathbb{C} \quad, \quad \tilde{u} \in C^{0}\left(W^{\prime}\right) \cap \mathcal{O}\left(W^{\prime \circ}\right)
$$


(onde $W^{\prime}=z\left(\overline{\mathcal{B}_{r}} \times \overline{J^{\prime}}\right)$ ) tal que $u=\tilde{u} \circ z$ em $\overline{\mathcal{B}_{r}} \times \overline{J^{\prime}}$. Note que o aberto $\mathcal{B}_{\mathrm{r}} \times J$ apenas precisa ser diminuído na variável $x$, com $J$ substituído por $J^{\prime}$, mas $r$ pode ser mantido o mesmo de antes.

Antes de mais nada, observe que, se $0 \in \mathcal{N}_{0}$, então $\overline{\mathcal{B}_{r}} \times\{0\}$ é uma fibra de $z$, e os campos $L_{j}$ se escrevem como $L_{j}=\partial_{t_{j}}$ ali. Sendo $L_{j} u=0$ para todo $j$, concluímos que $u$ é constante ao longo dessa fibra. Portanto, no restante da prova assuma que $0 \notin \mathcal{N}_{0}$.

Primeiro caso: Provemos o teorema inicialmente para $m=1$. Portanto aqui só existe uma variável $t$, e $\mathbb{L}$ é gerada por um único campo $L=\partial_{t}+\lambda \partial_{x}$. A ideia principal será provar que existe uma quantidade finita de abertos perto de 0 , cujas fronteiras são curvas analíticas, nos quais a função $z$ é invertível, e assim poderemos encontrar ũ como no enunciado simplesmente definindo-a como $u \circ z^{-1}$ nesses abertos e estendendo-a adequadamente.

Notemos que, para qualquer $x \in \overline{\mathrm{J}}$, se $x \notin \mathcal{N}_{0}$, então a função $\phi_{\mathrm{t}}(\cdot, x): \overline{\mathcal{B}_{\mathrm{r}}} \rightarrow \mathbb{R}$ tem uma quantidade finita de zeros. De fato, se houvesse infinitos zeros, eles acumulariam em $\overline{\mathcal{B}_{r}}$, e a função analítica $\phi_{\mathrm{t}}$ seria identicamente nula em $\overline{\mathcal{B}_{\mathrm{r}}} \times\{x\}$, isto é, $\phi$ seria constante ali. Mas como $\phi(0, x)=0$, teríamos $x \in \mathcal{N}_{0}$, contradição.

Dessa forma, se $\varepsilon>0$ é tal que $[-\varepsilon, \varepsilon] \subseteq \bar{J}$ não intercepta $\mathcal{N}_{0}$ ( $\varepsilon$ existe devido à finitude de $\mathcal{N}_{0}$ e ao fato que $0 \notin \mathcal{N}_{0}$ ), então $\phi_{\mathrm{t}}$ tem uma quantidade finita de zeros em cada hiperplano

$$
\mathrm{H}_{\mathrm{x}}=\left\{(\mathrm{t}, \mathrm{x}) ; \mathrm{t} \in \overline{\mathcal{B}_{\mathrm{r}}}\right\} \quad, \quad|x| \leq \varepsilon
$$

De acordo com o lema 2.11, podemos diminuir $\varepsilon$ de forma que, para cada natural $\mathrm{N}$, a quantidade de zeros de $\phi_{\mathrm{t}}(\cdot, x)$ em $\overline{\mathcal{B}_{\mathrm{r}}}$ com multiplicidade $N$ independa de $x \in(0, \varepsilon]$, e o mesmo pode ser feito para $x \in[-\varepsilon, 0)$. Seja $x \in(0, \varepsilon]$ arbitrário. Denote por $N^{\prime}$ a quantidade de zeros de $\phi_{\mathrm{t}}(\cdot, x)$ em $\overline{\mathcal{B}_{\mathrm{r}}}$ com multiplicidade impar, e denote estes zeros em ordem crescente por $\rho_{1}(x), \ldots, \rho_{N^{\prime}}(x)$. Defina também $\rho_{0}(x)=-r$ e $\rho_{N^{\prime}+1}(x)=r$. Dessa forma,

$$
\rho_{j}:(0, \varepsilon] \rightarrow \overline{\mathcal{B}_{\mathrm{r}}} \quad, \quad j=0, \ldots, \mathrm{N}^{\prime}+1
$$

são curvas analíticas que não se interceptam, e a função $\phi_{\mathrm{t}}(\cdot, x)$ troca de sinal em cada $\rho_{j}(x), j=1, \ldots, N^{\prime}$, o que implica que $\phi(\cdot, x)$ é estritamente monótona em cada intervalo

$$
\left[\rho_{j}(x), \rho_{j+1}(x)\right], \quad j=0, \ldots, N^{\prime}
$$

alternando entre crescente e decrescente em intervalos consecutivos. Agora defina, para cada $j=0, \ldots, N^{\prime}$,

$$
\begin{gathered}
A_{j}=\left\{(t, x) \in \overline{\mathcal{B}_{r}} \times(0, \varepsilon] ; \rho_{j}(x) \leq t \leq \rho_{j+1}(x)\right\} \\
B_{j}=z\left(A_{j}\right)=\left\{\zeta \in \mathbb{C} ; 0<\mathfrak{R} \zeta \leq \varepsilon, \Im \zeta \text { está entre } \phi\left(\rho_{j}(x), x\right) \text { e } \phi\left(\rho_{j+1}(x), x\right)\right\}
\end{gathered}
$$

Temos então que $z$ é um homeomorfismo de $A_{j}$ em $B_{j}$. Deve-se destacar que $\phi_{t}$ pode se anular no interior de $A_{j}$ (os pontos $\rho_{j}(x)$ são apenas seus zeros de multiplicidade impar), 
mas o conjunto de zeros dessa função ali é uma reunião de finitas curvas analíticas $\delta_{1}, \ldots, \delta_{K}$ onde $\phi_{\mathrm{t}}$ não muda de sinal, o que não afeta a monotonicidade de $\phi$. Definimos

$$
C_{j}=A_{j} \backslash \bigcup_{k=1}^{k} \delta_{k} \subseteq A_{j}
$$

Portanto, em $C_{j}$, a função $z$ é um difeomorfismo sobre sua imagem. Sejam ainda $\Gamma_{0}, \ldots, \Gamma_{\mathrm{N}^{\prime}+1}$ as curvas analíticas $z\left(\rho_{0}\right), \ldots, z\left(\rho_{N^{\prime}+1}\right) \subseteq W^{\prime}$. Como $\phi(\cdot, x)$ é estritamente monótona entre duas $\rho_{j}$ consecutivas, deduzimos que duas $\Gamma_{j}$ consecutivas não podem se interceptar, embora nada possa ser afirmado a respeito de interseções entre as $\Gamma_{j}$ em geral. Também notamos que, novamente pela monotonicidade de $\phi(\cdot, x)$, e pelo fato desta função alternar entre crescente e decrescente, dois conjuntos $B_{j}$ consecutivos têm interseção com interior não-vazio, e ainda $\Gamma_{j} \subseteq \partial\left(B_{j} \cap B_{j+1}\right)$.

Defina a função contínua

$$
\tilde{u}_{j}=u \circ z^{-1} \text { em } B_{j}, j=0, \ldots, N^{\prime}
$$

Afirmamos que $\tilde{u}_{j}$ é holomorfa em $B_{j}^{\circ}$. De fato, observe que

$$
0=\mathrm{Lu}=\mathrm{L}\left(\tilde{u}_{j} \circ z\right)=\frac{\partial \tilde{u}_{j}}{\partial \zeta}(z) \mathrm{L} z+\frac{\partial \tilde{u}_{j}}{\partial \bar{\zeta}}(z) \mathrm{L} \bar{z}=\frac{\partial \tilde{u}_{j}}{\partial \bar{\zeta}}(z) \mathrm{L} \bar{z}
$$

Mas por outro lado

$$
\mathrm{L} \bar{z}=\mathrm{L} \bar{z}-\overline{\mathrm{Lz}}=(\mathrm{L}-\overline{\mathrm{L}}) \bar{z}=-2 i(\mathfrak{I} \mathrm{L}) \bar{z}=-2 i \lambda \frac{\partial \bar{z}}{\partial x}=\frac{-2 i \phi_{\mathrm{t}}}{1+\phi_{\mathrm{x}}} \frac{\partial \bar{z}}{\partial x}
$$

E assim

$$
0=\frac{\partial \tilde{u}_{j}}{\partial \bar{\zeta}}(z(t, x)) \frac{-2 i \phi_{t}(t, x)}{1+\phi_{x}(t, x)} \frac{\partial \bar{z}}{\partial x}(t, x)
$$

Como $\bar{z}_{x}$ nunca se anula e $\phi_{t}$ não se anula em $(t, x) \in C_{j}$, obtemos que

$$
\frac{\partial \tilde{u}_{j}}{\partial \bar{\zeta}}(z(t, x))=0 \quad, \quad(t, x) \in C_{j}
$$

Isto é, $\tilde{u}_{j}$ é holomorfa em $z\left(C_{j}\right)$. Agora, como $B_{j}^{\circ}$ é a reunião de $z\left(C_{j}\right)$ com uma quantidade finita de curvas analíticas (as curvas $z\left(\delta_{1}\right), \ldots, z\left(\delta_{k}\right)$ ) - um conjunto analítico - concluímos por continuidade e pelo lema 2.12 que $\tilde{u}_{j}$ é holomorfa em $B_{j}^{\circ}$, como queríamos.

Nosso próximo passo é "colar" todas as $\tilde{u}_{j}$. As funções holomorfas $\tilde{u}_{j}$ e $\tilde{u}_{j+1}$ estão ambas definidas no aberto $\left(B_{j} \cap B_{j+1}\right)^{\circ}$ e coincidem em $\Gamma_{j}=z\left(\rho_{j}\right)$, pois $\rho_{j}$ está na fronteira de $A_{j}$ e $A_{j+1}$. Concluímos do principio de identidade de funções holomorfas que elas coincidem em $B_{j} \cap B_{j+1}$., o que é suficiente para garantir que existe $\tilde{u}$ holomorfa em $\cup_{j=0, \ldots, N^{\prime}} B_{j}$ satisfazendo

$$
u=\tilde{u} \circ z \quad \operatorname{em} \overline{\mathcal{B}_{r}} \times(0, \varepsilon]
$$


Se fizermos o mesmo processo para $\chi$ negativo (possivelmente com outro valor de $N^{\prime}$ ) e chamarmos também de ũ a função obtida, a conclusão é que

$$
\tilde{\mathrm{u}} \in \mathrm{C}^{0}\left(\overline{\mathcal{B}_{\mathrm{r}}} \times([-\varepsilon, \varepsilon] \backslash\{0\})\right) \cap \mathcal{O}\left(\mathcal{B}_{\mathrm{r}} \times((-\varepsilon, \varepsilon) \backslash\{0\})\right)
$$

$\mathrm{e}$

$$
u(t, x)=\tilde{u}(z(t, x)) \quad, \quad 0<|x| \leq \varepsilon
$$

O trabalho agora é estender $\tilde{u}$ holomorficamente ao hiperplano $x=0$ de forma que a igualdade acima continue válida. Existem finitos pontos $\left(t^{(1)}, 0\right), \ldots,\left(t^{(p)}, 0\right)$ neste hiperplano nos quais $\phi_{\mathrm{t}}$ se anula. Se $(\mathrm{t}, 0)$ não é um deles, pelo Teorema da Função Inversa, $z$ é um difeomorfismo de uma vizinhança aberta de $(t, 0)$ em uma vizinhança aberta $Z$ de $z(t, 0)$, que é um ponto do eixo imaginário. Podemos definir $\tilde{v}=u \circ z^{-1}$ em $Z$, e ela será holomorfa ali, pelo mesmo cálculo que fizemos em (2.14) acima. Mas como ṽ coincide com $\tilde{u}$ onde esta está definida em $Z$, ou seja, no aberto $\{\zeta \in Z ; \mathfrak{R} \zeta \neq 0\}$, temos que $\tilde{v}$ é uma extensão holomorfa para ũ. Concluímos então que ũ se estende continuamente a $W^{\prime}$ e holomorficamente a $W^{\prime \circ}$, exceto possivelmente nos pontos $z\left(t^{(1)}, 0\right), \ldots, z\left(t^{(p)}, 0\right)$. Mas estes, por serem em número finito, seriam polos de ũ caso esta não pudesse ser estendida holomorficamente a eles, o que não são porque ũ é limitada. (Essa limitação vem da equação $u=\tilde{u} \circ z$ em $\mathfrak{R} \zeta \neq 0$ ). Então $\tilde{u}$ está de fato definida continuamente em $W^{\prime}$ e holomorficamente em $W^{\prime \circ}$. A igualdade $u=\tilde{u} \circ z$ vale também em $\{x=0\}$ por continuidade.

Segundo caso: Agora suponha que $m \in \mathbb{N}$ é qualquer. Provaremos em um primeiro momento que $u$ é constante nas fibras de $z$ em $\mathcal{B}_{\mathrm{r}} \times[-\varepsilon, \varepsilon]$, para algum $\varepsilon>0$. A partir disto será simples provar que existe $\tilde{u}$ como no enunciado.

Sejam então $z_{0}=x_{0}+i y_{0} \in W e\left(t^{(1)}, x_{0}\right),\left(t^{(2)}, x_{0}\right) \in \overline{\mathcal{B}_{r}}$ tais que $z\left(t^{(1)}, x_{0}\right)=z\left(t^{(2)}, x_{0}\right)=$ $z_{0}$. Queremos provar que $u\left(t^{(1)}, x_{0}\right)=u\left(t^{(2)}, x_{0}\right)$, desde que $\left|x_{0}\right|$ seja suficientemente pequeno. Tome uma curva analítica $\gamma=\left(\gamma^{1}, \ldots, \gamma^{\mathrm{m}}\right):[-1,1] \rightarrow \overline{\mathcal{B}_{\mathrm{r}}}$ tal que $\gamma^{\prime} \neq 0 \mathrm{e}$

$$
\gamma(-1)=\mathrm{t}^{(1)}, \gamma(0)=0, \gamma(1)=\mathrm{t}^{(2)}
$$

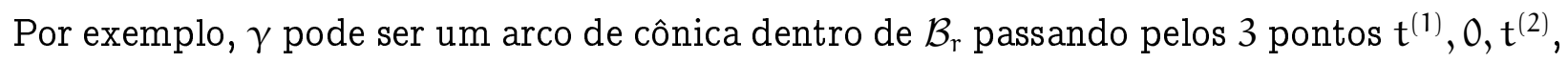
e parametrizado de forma a obter as equações acima. Denote por $\mathcal{M}$ a variedade analítica $\gamma \times \overline{\mathrm{J}}$, imersa em $\overline{\mathcal{B}_{\mathrm{r}}} \times \overline{\mathrm{J}}$ pela aplicação

$$
\sigma:(s, x) \mapsto(\gamma(s), x)
$$

Defina em $[-1,1] \times \overline{\mathrm{J}}$ as funções

$$
\phi^{\sharp}=\phi \circ \sigma \quad, \quad u^{\sharp}=u \circ \sigma, \quad z^{\sharp}=z \circ \sigma
$$

Considere também o campo de vetores

$$
L=\sum_{j=1}^{m} \frac{d \gamma^{j}}{d s}(s) L_{j}=\sum_{j=1}^{m} \frac{d \gamma^{j}}{d s}(s) \frac{\partial}{\partial t_{j}}-\left(\frac{i}{1+i \phi_{x}(\gamma(s), x)} \sum_{j=1}^{m} \frac{d \gamma^{j}}{d s}(s) \phi_{t_{j}}(\gamma(s), x)\right) \frac{\partial}{\partial x}
$$


Em particular, sua direção nas variáveis t é a mesma da curva $\gamma$, e portanto L está definido em $\mathcal{M}$. Seu pullback à variedade $[-1,1] \times \overline{\mathrm{J}}$ é dado por

$$
L^{\sharp}=\frac{\partial}{\partial s}-\frac{i \phi_{s}^{\sharp}}{1+i \phi_{x}^{\sharp}} \frac{\partial}{\partial x}
$$

Isto é, $L^{\sharp}$ é o campo que satisfaz $L^{\sharp}(f \circ \sigma)=(\mathrm{L} f) \circ \sigma$ para toda função $f$ em $\overline{\mathcal{B}_{r}} \times[-\varepsilon, \varepsilon]$. Em particular, $L^{\sharp} u^{\sharp}=(\mathrm{Lu}) \circ \sigma=0$ e similarmente $L^{\sharp} z^{\sharp}=0$. Assim $z^{\sharp}$ é uma integral primeira para a estrutura gerada por $L^{\sharp}$, e como $[-1,1] \times \bar{J}$ é uma variedade 2-dimensional e o campo $\mathrm{L}^{\sharp}$ tem o mesmo formato do campo que gerava a estrutura no caso $\mathrm{m}=1$ acima, podemos aplicar este mesmo teorema para $m=1$ e $r=1$ e assim obter $\varepsilon>0$ tal que $u^{\sharp}$ é constante nas fibras de $z^{\sharp}$ em $[-1,1] \times[-\varepsilon, \varepsilon]$. Em particular, se $\circ x_{0}$ do início fosse tomado com $\left|x_{0}\right| \leq \varepsilon$, então, tendo-se em vista que

$$
z^{\sharp}\left(-1, x_{0}\right)=z\left(\gamma(-1), x_{0}\right)=z\left(t^{(1)}, x_{0}\right)=z\left(t^{(2)}, x_{0}\right)=z\left(\gamma(1), x_{0}\right)=z^{\sharp}\left(1, x_{0}\right)
$$

concluímos

$$
u\left(t^{(1)}, x_{0}\right)=u\left(\gamma(-1), x_{0}\right)=u^{\sharp}\left(-1, x_{0}\right)=u^{\sharp}\left(1, x_{0}\right)=u\left(\gamma(1), x_{0}\right)=u\left(t^{(2)}, x_{0}\right)
$$

conforme queríamos demonstrar.

Fica assim bem-definida a função $\tilde{u}: H=z\left(\overline{\mathcal{B}_{r}} \times[-\varepsilon, \varepsilon]\right) \rightarrow \mathbb{C}$ dada por

$$
\tilde{u}(z(t, x))=u(t, x) \quad, \quad(t, x) \in \overline{\mathcal{B}_{r}} \times[-\varepsilon, \varepsilon]
$$

e falta provar que ela é contínua em $\mathrm{H}$ e holomorfa em $\mathrm{H}^{\circ}$.

A continuidade segue por Topologia: seja $V \subseteq \mathbb{C}$ aberto. Como u é contínua, a imagem inversa $u^{-1}(V)$ é aberta em $\overline{\mathcal{B}_{r}} \times[-\varepsilon, \varepsilon]$. Denote por $\sim$ a relação de equivalência em $\overline{\mathcal{B}_{\mathrm{r}}} \times[-\mathcal{E}, \varepsilon]$ dada pelas fibras de $z$, isto é,

$$
\left(\mathrm{t}^{(1)}, x^{(1)}\right) \sim\left(\mathrm{t}^{(2)}, x^{(2)}\right) \Longleftrightarrow z\left(\mathrm{t}^{(1)}, x^{(1)}\right)=z\left(\mathrm{t}^{(2)}, x^{(2)}\right)
$$

e seja $\pi$ a aplicação quociente desta relação. Então $\pi$ é aberta, e assim $\pi\left(u^{-1}(V)\right)$ é aberto em $\overline{\mathcal{B}_{\mathrm{r}}} \times[-\varepsilon, \varepsilon] / \sim$. Um teorema padrão de Topologia garante a existência de uma função contínua injetiva

$$
\hat{z}: \frac{\overline{\mathcal{B}_{\mathrm{r}}} \times[-\varepsilon, \varepsilon]}{\sim} \rightarrow \mathbb{C}
$$

tal que $z=\hat{z} \circ \pi$. Mas como o domínio de $\hat{z}$ é compacto, ela é um homeomorfismo sobre sua imagem, e concluímos que $z\left(u^{-1}(V)\right)=\hat{z}\left(\pi\left(u^{-1}(V)\right)\right)$ é aberto em H. Sendo $u=\tilde{u} \circ z$, isto é o mesmo que dizer que $\tilde{u}^{-1}(V)$ é aberto, e então ũ é contínua.

Para provarmos que $\tilde{u} \in \mathcal{O}\left(\mathrm{H}^{\circ}\right)$, fixe um ponto $\zeta_{0}=x_{0}+i y_{0} \in \mathrm{H}^{\circ}$. Seja $\gamma:[-1,1] \rightarrow \overline{\mathcal{B}_{\mathrm{r}}}$ uma curva analítica tal que $\gamma^{\prime} \neq 0$ e $y_{0}=\phi\left(\gamma(0), x_{0}\right)$, por exemplo um segmento de reta. Defina $\mathcal{M}, \sigma, u^{\sharp}, z^{\sharp}$ como fizemos acima, e $H^{\sharp}=z^{\sharp}([-1,1] \times \bar{J}) \subseteq H$. Observe que $z_{0}$ pertence 
ao interior da 1-variedade $H^{\sharp}$, que denotamos como $\left(H^{\sharp}\right)^{\circ}$ (não se trata do interior de $H^{\sharp}$ como subespaço de $\mathrm{H}$; este seria nulo). Pelo caso $m=1$, existe uma função

$$
\tilde{u}^{\sharp}: H^{\sharp} \rightarrow \mathbb{C} \quad, \quad \tilde{u^{\sharp}} \in C^{0}\left(H^{\sharp}\right) \cap \mathcal{O}\left(\left(H^{\sharp}\right)^{\circ}\right)
$$

tal que $u^{\sharp}=\tilde{u}^{\sharp} \circ z^{\sharp}$. Em particular, $u^{\sharp}$ é holomorfa em $z_{0}$.

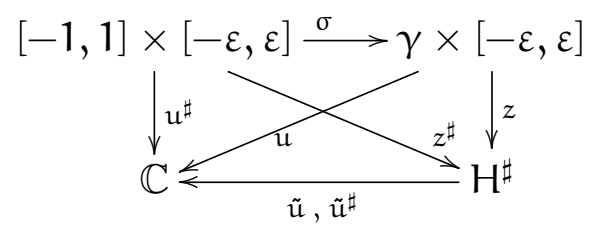

Mas por outro lado, temos $u^{\sharp}=u \circ \sigma=(\tilde{u} \circ z) \circ \sigma=\tilde{u} \circ z^{\sharp}$, $\circ$ que implica que $\tilde{u}^{\sharp}=\tilde{u}$, e assim $u$ é holomorfa em $z_{0}$, conforme queríamos.

Se aplicamos este resultado para comparar duas integrais primeiras em torno de um mesmo ponto, obtemos:

Proposição 2.15. Sejam $z, w$ duas integrais primeiras de $\mathbb{L}$ num aberto $\mathrm{U}$ em torno de $\omega_{0}$. Então $\omega_{0}$ admite uma vizinhança aberta $\mathrm{V} \subseteq \mathrm{U}$ tal que

$$
z=\mathrm{h} \circ \mathrm{w} \quad \text { em } \mathrm{V}
$$

para alguma função contínua $\mathrm{h}: w(\mathrm{~V}) \rightarrow z(\mathrm{~V})$, biholomorfismo se restrita $a w(\mathrm{~V})^{\circ} \rightarrow$ $z(\mathrm{~V})^{\circ}$.

Demonstração: Pelo teorema 2.13 acima, existem abertos $V_{1}, V_{2} \ni \omega_{0}$ tais que

$$
\begin{array}{ll}
z=\tilde{z} \circ w & \text { em } V_{1} \\
w=\tilde{w} \circ z & \text { em } V_{2}
\end{array}
$$

para certas funções contínuas $\tilde{z}$ e $\tilde{w}$, holomorfas em $z\left(V_{1}\right)^{\circ}$ e $w\left(V_{2}\right)^{\circ}$. Considere as restrições de $z, w$ a $V=V_{1} \cap V_{2}$. Então $z=\tilde{z} \circ(\tilde{w} \circ z) \Rightarrow \tilde{z} \circ \tilde{w}=\mathrm{id}$, e analogamente $\tilde{w} \circ \tilde{z}=\mathrm{id}$. Assim $\tilde{z}$ e $\tilde{w}$ são inversas uma da outra, e portanto biholomorfismos entre os conjuntos $z(V)^{\circ}$ e $w(V)^{\circ}$.

Corolário 2.18. Se uma integral primeira de $\mathbb{L}$ em torno de $\omega_{0}$ é aberta em $\omega_{0}$, então qualquer integral primeira de $\mathbb{L}$ em torno de $\omega_{0}$ também o é.

Definição 2.19. Dizemos que a estrutura $\mathbb{L}$ é aberta em $\omega_{0} \in \Omega$ se existe alguma integral primeira em torno de $\omega_{0}$ aberta nesse ponto, ou equivalentemente se todas as integrais primeiras em torno de $\omega_{0}$ são abertas nesse ponto.

A proposição 2.15 tem uma importante consequência: a noção de fibra de uma integral primeira $z$ em torno de um ponto independe da escolha de $z$. De fato, a equação (2.16) diz que as fibras de $w$ em $V_{1} \cap V_{2}$ estão contidas nas de $z$, e a equação (2.17) prova que as fibras de $z$ estão contidas nas de $w$. Fica então bem definido o conceito: 
Definição 2.20. Uma fibra de $\mathbb{L}$ em torno de $\omega_{0}$ é um conjunto de nivel de qualquer integral primeira de $\mathbb{L}$ numa vizinhança aberta de $\omega_{0}$.

\subsection{Soluções distribucionais}

O segundo resultado mais importante de [BT] lida com as soluções distribucionais de $\mathbb{L}$. Provaremos que, localmente, elas são dadas na forma Pw, para algum operador diferencial parcial linear $\mathrm{P}$ com coeficientes analíticos e alguma solução contínua $w$. Obteremos $w$ através de Análise de Fourier e propriedades dos espaços de Sobolev, mas precisaremos para isso trabalhar com uma variação deste último conceito que tire proveito do fato que os campos $L_{j}$ são elípticos apenas nas variáveis t. Aqui daremos o nome de espaços de Sobolev parciais a essa variação. Nossa abordagem para tanto foi inspirada em [T1], proposição 5.2, p. 39, e [T2], proposição I.4.3, p. 25, onde o autor apresenta a definição destes espaços.

Denotaremos por $\mathcal{F} f$ ou por $\hat{f}$ a transformada de Fourier de uma distribuição temperada $f$, e os pontos de seu domínio $\mathbb{R}^{m+1}$ serão escritos como $(\xi, \eta)$, com $\xi \in \mathbb{R}^{m}$ e $\eta \in \mathbb{R}$. A notação f̆ será empregada para a transformada inversa.

Definição 2.21. Dado $\mathrm{p} \in \mathbb{R}$, o espaço de Sobolev $\mathrm{H}^{\mathrm{p}}\left(\mathbb{R}^{\mathrm{m}+1}\right)$, denotado simplesmente por $\mathrm{H}^{\mathrm{p}}$, é definido por

$$
\begin{aligned}
& \mathrm{H}^{\mathrm{p}}=\left\{\mathrm{f} \in \mathcal{D}^{\prime}\left(\mathbb{R}^{\mathrm{m}+1}\right) ; \hat{\mathrm{f}}\right. \text { é uma função mensurável e } \\
& \left.\qquad\|\mathrm{f}\|_{\mathrm{H}^{\mathrm{p}}}^{2}=\int_{\mathbb{R}} \int_{\mathbb{R}^{\mathrm{m}}}\left(1+|\xi|^{2}+\eta^{2}\right)^{\mathrm{p}}|\hat{\mathrm{f}}(\xi, \eta)|^{2} \mathrm{~d} \xi \mathrm{d} \eta<\infty\right\}
\end{aligned}
$$

Espaços de Sobolev são espaços vetoriais, e vale a seguinte relação de inclusão:

$$
\mathrm{H}^{\mathrm{p}_{1}} \subseteq \mathrm{H}^{\mathrm{p}_{2}} \Longleftrightarrow \mathrm{p}_{1} \geq \mathrm{p}_{2}
$$

Definição 2.22. Dados $\Omega^{\prime} \subseteq \mathbb{R}^{m+1}$ aberto e $p \in \mathbb{R}$, o espaço de Sobolev local $\mathrm{H}_{\text {loc }}^{\mathrm{p}}\left(\Omega^{\prime}\right)$ é definido por

$$
\mathrm{H}_{\mathrm{loc}}^{\mathrm{p}}\left(\Omega^{\prime}\right)=\left\{\mathrm{f} \in \mathcal{D}^{\prime}\left(\Omega^{\prime}\right) ; \chi \mathrm{f} \in \mathrm{H}^{\mathrm{p}} \text { para toda } \chi \in \mathrm{C}_{\mathrm{c}}^{\infty}\left(\Omega^{\prime}\right)\right\}
$$

Dizer que $\chi f \in H^{p}$ é um abuso de notação, já que a rigor a distribuição $\chi f$ está definida apenas em $\Omega^{\prime}$. Entretanto, como essa distribuição claramente se estende (como zero) para fora de $\Omega^{\prime}$, podemos considerá-la como definida em $\mathbb{R}^{\mathrm{m}+1}$.

Espaços de Sobolev locais também são espaços vetoriais, e vale a mesma relação de inclusão apresentada acima. Listamos abaixo outras de suas propriedades bem conhecidas:

- $f \in H^{p}, \chi \in C_{c}^{\infty}\left(\mathbb{R}^{m+1}\right) \Rightarrow \chi f \in H^{p}$ 
- $f \in \mathrm{H}_{\mathrm{loc}}^{\mathrm{p}}\left(\Omega^{\prime}\right), \chi \in \mathrm{C}^{\infty}\left(\Omega^{\prime}\right) \Rightarrow \chi f \in \mathrm{H}_{\mathrm{loc}}^{\mathrm{p}}\left(\Omega^{\prime}\right)$

- $f \in H_{\text {loc }}^{p}\left(\Omega^{\prime}\right)$ se e somente se, para cada aberto $W \subset \subset \Omega^{\prime}$, existe $g \in H^{p}$ tal que $\mathrm{g}=\mathrm{f}$ em W.

- $\bigcap_{\mathrm{p} \in \mathbb{R}} \mathrm{H}_{\mathrm{loc}}^{\mathrm{p}}\left(\Omega^{\prime}\right)=\mathrm{C}^{\infty}\left(\Omega^{\prime}\right)$

- Se $\Omega^{\prime} \subset \subset \Omega$ são abertos e $f \in \mathcal{D}^{\prime}(\Omega)$, então existe $p \in \mathbb{R}$ tal que a restrição $\left.f\right|_{\Omega^{\prime}} \in \mathcal{D}^{\prime}\left(\Omega^{\prime}\right)$ pertence a $H_{\text {loc }}^{p}\left(\Omega^{\prime}\right)$.

Definição 2.23. Dados $r, s \in \mathbb{R}$, o espaço de Sobolev parcial $H^{r, s}\left(\mathbb{R}^{m+1}\right)$, denotado simplesmente por $\mathrm{H}^{\mathrm{r}, \mathrm{s}}$, é definido por

$$
H^{r, s}=\left\{f \in \mathcal{D}^{\prime}\left(\mathbb{R}^{m+1}\right) ; \hat{f} \text { é uma função mensurável } e\right.
$$

$$
\left.\|f\|_{H^{r, s}}^{2}=\int_{\mathbb{R}} \int_{\mathbb{R}^{m}}\left(1+|\xi|^{2}\right)^{r}\left(1+\eta^{2}\right)^{s}|\hat{f}(\xi, \eta)|^{2} d \xi d \eta<\infty\right\}
$$

Dados $\Omega^{\prime} \subseteq \mathbb{R}^{m+1}$ aberto e $r, s \in \mathbb{R}$, o espaço de Sobolev parcial local $\mathrm{H}_{\text {loc }}^{\mathrm{r}, \mathrm{s}}\left(\Omega^{\prime}\right)$ é definido por

$$
\mathrm{H}_{\mathrm{loc}}^{\mathrm{r}, \mathrm{s}}\left(\Omega^{\prime}\right)=\left\{\mathrm{f} \in \mathcal{D}^{\prime}\left(\Omega^{\prime}\right) ; \chi \mathrm{f} \in \mathrm{H}^{\mathrm{r}, \mathrm{s}} \text { para toda } \chi \in \mathrm{C}_{\mathrm{c}}^{\infty}\left(\Omega^{\prime}\right)\right\}
$$

Naturalmente, espaços de Sobolev parciais e seus análogos locais são espaços vetoriais, e valem as seguintes relações de inclusão:

$$
\mathrm{r}_{1} \geq \mathrm{r}_{2}, \mathrm{~s}_{1} \geq \mathrm{s}_{2} \Rightarrow \mathrm{H}^{\mathrm{r}_{1}, \mathrm{~s}_{1}} \subseteq \mathrm{H}^{\mathrm{r}_{2}, \mathrm{~s}_{2}}, \mathrm{H}_{\mathrm{loc}}^{\mathrm{r}_{1}, \mathrm{~s}_{1}} \subseteq \mathrm{H}_{\mathrm{loc}}^{\mathrm{r}_{2}, \mathrm{~s}_{2}}
$$

Mas em geral não podemos comparar esses espaços se por exemplo $r_{1}>r_{2}$ e $s_{1}<s_{2}$.

Proposição 2.24. Se $\mathrm{r}, \mathrm{s} \geq 0$, então $\mathrm{H}^{\mathrm{r}+\mathrm{s}} \subseteq \mathrm{H}^{\mathrm{r}, \mathrm{s}}$.

Demonstração: Basta notarmos que, para A, B não-negativos,

$$
\frac{(1+A)^{r}(1+B)^{s}}{(1+A+B)^{r+s}}=\frac{(1+A)^{r}}{(1+A+B)^{r}} \frac{(1+B)^{s}}{(1+A+B)^{s}}=\left(\frac{1+A}{1+A+B}\right)^{r}\left(\frac{1+B}{1+A+B}\right)^{s} \leq 1
$$

Logo, se $f \in \mathrm{H}^{\mathrm{r}+\mathrm{s}}$, temos

$$
\begin{aligned}
& \iint\left(1+|\xi|^{2}\right)^{r}\left(1+\eta^{2}\right)^{s}|\hat{f}(\xi, \eta)|^{2} d \xi d \eta= \\
& \quad=\iint \frac{\left(1+|\xi|^{2}\right)^{r}\left(1+\eta^{2}\right)^{s}}{\left(1+|\xi|^{2}+\eta^{2}\right)^{r+s}}\left(1+|\xi|^{2}+\eta^{2}\right)^{r+s}|\hat{f}(\xi, \eta)|^{2} d \xi d \eta<\infty
\end{aligned}
$$

Proposição 2.25. Dado $\mathrm{p} \in \mathbb{R}$, existem $\mathrm{r}, \mathrm{s} \in \mathbb{R}$ tais que $\mathrm{H}^{\mathrm{p}} \subseteq \mathrm{H}^{\mathrm{r}, \mathrm{s}}$. 
Demonstração: Se $p \geq 0$, pela proposição acima podemos afirmar que $H^{p} \subseteq H^{p, 0}$. Se $p \leq 0$, observe que, para $A, B$ não-negativos,

$$
\frac{(1+A)^{p}(1+B)^{p}}{(1+A+B)^{p}}=\left(\frac{1+A+B+A B}{1+A+B}\right)^{p} \leq 1^{p}=1
$$

O mesmo cálculo com integrais da proposição anterior mostra então que $\mathrm{H}^{\mathfrak{p}} \subseteq \mathrm{H}^{\mathrm{p}, \mathrm{p}}$.

Lema 2.26. (Desigualdade de Peetre) Sejam $N \in \mathbb{N}$ e $u, v, w \in \mathbb{R}^{N} \operatorname{com} u+v=w$. Seja ainda $\mathrm{p} \in \mathbb{R}$. Então

$$
\left(1+|w|^{2}\right)^{p} \leq 2^{|p|}\left(1+|u|^{2}\right)^{p}\left(1+|v|^{2}\right)^{|p|}
$$

Demonstração: Como $|w|=|u+v| \leq|u|+|v|$, elevando ao quadrado e observando que $|u v| \leq|u|^{2}+|v|^{2}$, temos $|w|^{2} \leq 2\left(|u|^{2}+|v|^{2}\right)$. Em particular

$$
\left(1+|w|^{2}\right) \leq 1+2\left(|u|^{2}+|v|^{2}\right)<2\left(1+|u|^{2}\right)\left(1+|v|^{2}\right)
$$

Se $p \geq 0$, segue imediatamente que

$$
\left(1+|w|^{2}\right)^{p} \leq 2^{p}\left(1+|u|^{2}\right)^{p}\left(1+|v|^{2}\right)^{p}
$$

como queríamos. Se $p \leq 0$, como $u=w+(-v)$ podemos aplicar a equação (2.27) acima fazendo as substituições $w \leftrightarrow u, u \leftrightarrow w$ e $v \leftrightarrow-v$ :

$$
\left(1+|u|^{2}\right)<2\left(1+|w|^{2}\right)^{p}\left(1+|v|^{2}\right)^{p}
$$

E portanto

$$
\left(1+|u|^{2}\right)^{p} \geq 2^{p}\left(1+|w|^{2}\right)^{p}\left(1+|v|^{2}\right)^{p} \Rightarrow\left(1+|w|^{2}\right)^{p} \leq 2^{-p}\left(1+|u|^{2}\right)^{p}\left(1+|v|^{2}\right)^{-p}
$$

Como $|p|=-p$, esta é de novo a equação que queríamos.

Lema 2.28. (Desigualdade de Minkowski integral) Sejam (X, dx) e (, $\mathrm{dy})$ espaços de medida $\sigma$-finitos. Então, para qualquer $\mathrm{p} \in[1, \infty)$ e qualquer função $\mathrm{g}: \mathrm{X} \times \mathrm{Y} \rightarrow \mathbb{C}$ mensurável com relação a $\mathrm{d} x \times \mathrm{d} y$,

$$
\left(\int\left|\int g(x, y) d x\right|^{p} d y\right)^{1 / p} \leq \int\left(\int|g(x, y)|^{p} d y\right)^{1 / p} d x
$$

quer estes números sejam finitos ou não.

A demonstração deste lema pode ser encontrada em [F2], teorema 6.19, p. 194.

O próximo lema demonstra uma propriedade dos espaços de Sobolev parciais que é análoga à já conhecida para os usuais: o espaço $\mathrm{C}_{\mathrm{c}}^{\infty}$ é um multiplicador de $\mathrm{H}^{\mathrm{r}, \mathrm{s}}$. Com isso concluiremos também que o espaço $C^{\infty}$ é um multiplicador de $H_{l o c}^{\text {r,s }}$. 
Lema 2.29. Sejam $r, s \in \mathbb{R}$ e $f \in \mathcal{D}^{\prime}\left(\mathbb{R}^{m+1}\right)$. Se $f \in H^{r, s}$ e $\chi \in C_{c}^{\infty}\left(\mathbb{R}^{m+1}\right)$, então $\chi f \in H^{r, s}$.

Demonstração: Lembremos que a transformada de um produto é dada em termos da convolução das transformadas:

$$
\widehat{\chi^{f}}(\xi, \eta)=\frac{1}{(2 \pi)^{m+1}}(\hat{\chi} * \hat{f})(\xi, \eta)=\frac{1}{(2 \pi)^{m+1}} \iint \hat{\chi}(t, x) \hat{f}(\xi-t, \eta-x) d t d x
$$

Seja $\sigma<0$ tal que

$$
\int_{\mathbb{R}^{m}}\left(1+|t|^{2}\right)^{\frac{|r|}{2}+\frac{\sigma}{2}} d t<\infty \quad, \quad \int_{\mathbb{R}}\left(1+x^{2}\right)^{\frac{|s|}{2}+\frac{\sigma}{2}} d x<\infty
$$

Como $\chi \in \mathrm{C}_{\mathrm{c}}^{\infty}\left(\mathbb{R}^{\mathrm{m}+1}\right) \subseteq \mathcal{S}\left(\mathbb{R}^{\mathrm{m}+1}\right)$, temos $\hat{\chi} \in \mathcal{S}\left(\mathbb{R}^{\mathrm{m}+1}\right.$ ) (onde $\mathcal{S}$ denota o espaço das funções de Schwartz), e então existe $\mathrm{C}>0$ tal que

$$
|\hat{\chi}(t, x)| \leq C\left(1+|t|^{2}+x^{2}\right)^{\sigma} \quad, \quad(t, x) \in \mathbb{R}^{m+1}
$$

Agora podemos estimar:

$$
\begin{aligned}
\|\chi f\|_{H^{r, s}} & =\left(\iint\left(1+|\xi|^{2}\right)^{r}\left(1+\eta^{2}\right)^{s}\left|\iint \hat{\chi}(t, x) \hat{f}(\xi-t, \eta-x) d t d x\right|^{2} d \xi d \eta\right)^{1 / 2} \\
& \leq\left(\iint\left(1+|\xi|^{2}\right)^{r}\left(1+\eta^{2}\right)^{s}\left(\iint|\hat{\chi}(t, x) \hat{f}(\xi-t, \eta-x)| d t d x\right)^{2} d \xi d \eta\right)^{1 / 2} \\
& \stackrel{(2.28)}{\leq} \iint\left(\iint\left(1+|\xi|^{2}\right)^{r}\left(1+\eta^{2}\right)^{s}|\hat{\chi}(t, x)|^{2}|\hat{f}(\xi-t, \eta-x)|^{2} d \xi d \eta\right)^{1 / 2} d t d x \\
& \stackrel{(2.31)}{\leq} C \iint\left(\iint\left(1+|\xi|^{2}\right)^{r}\left(1+\eta^{2}\right)^{s} \cdot\right. \\
& \left.\cdot\left(1+|t|^{2}+x^{2}\right)^{2 \sigma}|\hat{f}(\xi-t, \eta-x)|^{2} d \xi d \eta\right)^{1 / 2} d t d x \\
& \leq C \iint\left(\iint\left(1+|t|^{2}\right)^{|r|}\left(1+|\xi-t|^{2}\right)^{r}\left(1+x^{2}\right)^{|s|}\left(1+(\eta-x)^{2}\right)^{s} \cdot\right. \\
& \left.=\left(1+|t|^{2}+x^{2}\right)^{2 \sigma}|\hat{f}(\xi-t, \eta-x)|^{2} d \xi d \eta\right)^{1 / 2} d t d x
\end{aligned}
$$

Observe que a Desigualdade de Minkowski integral foi aplicada às medidas $\mathrm{dt} \times \mathrm{d} x \mathrm{e}$ $\left(1+|\xi|^{2}\right)^{\mathrm{r}} \mathrm{d} \xi \times\left(1+\eta^{2}\right)^{\mathrm{s}} \mathrm{d} \eta$. Para terminar, note que

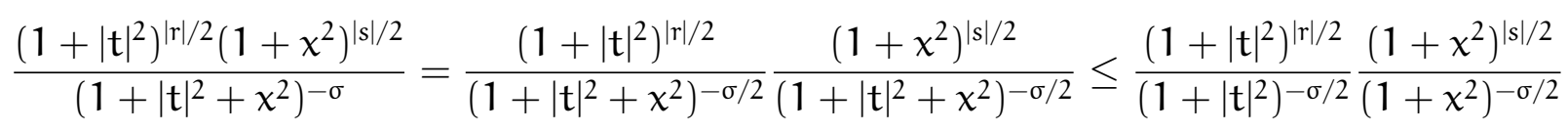

já que $\sigma<0$. Essa expressão, pelo Teorema de Fubini e pela nossa escolha de $\sigma$ em (2.30), é integrável. 
Corolário 2.32. Sejam $r, s \in \mathbb{R}, \Omega^{\prime} \subseteq \mathbb{R}^{m+1}$ aberto e $f \in \mathcal{D}^{\prime}\left(\Omega^{\prime}\right)$. Então $f \in \mathrm{H}_{\text {loc }}^{\mathrm{r}, \mathrm{s}}\left(\Omega^{\prime}\right)$ se e somente se, para cada aberto $\mathrm{W} \subset \subset \Omega^{\prime}$, existe $\mathrm{g} \in \mathrm{H}^{\mathrm{r}, \mathrm{s}}$ tal que $\mathrm{g}=\mathrm{f}$ em $\mathrm{W}$.

Demonstração: Suponha que $f \in \mathrm{H}_{\text {loc }}^{r, s}\left(\Omega^{\prime}\right)$, e seja $W \subset \subset \Omega^{\prime}$ aberto. Tome $\chi \in C_{c}^{\infty}\left(\Omega^{\prime}\right)$ tal que $\chi \equiv 1$ em $W$. Por hipótese, a distribuição $g=\chi f$, que coincide com $f$ em $W$, pertence a $\mathrm{H}^{\mathrm{r}, \mathrm{s}}$. Reciprocamente, suponha que para cada aberto $W \subset \subset \Omega^{\prime}$ existe $\mathrm{g}_{W} \in \mathrm{H}^{\mathrm{r}, \mathrm{s}}$ tal que $g_{W}=f$ em $W$, e seja $\chi \in C_{c}^{\infty}\left(\Omega^{\prime}\right)$. Tome um aberto $W \subset \subset \Omega^{\prime}$ contendo supp $\chi$. Pelo lema 2.29, a distribuição $\chi g_{W}$, que é igual a $\chi f$, pertence a $H^{r, s}$.

Corolário 2.33. Sejam $r, s \in \mathbb{R}, \Omega^{\prime} \subseteq \mathbb{R}^{m+1}$ aberto e $f \in \mathcal{D}^{\prime}\left(\Omega^{\prime}\right)$. Se $f \in \mathrm{H}_{\text {loc }}^{r, s}\left(\Omega^{\prime}\right)$ e $\chi \in \mathrm{C}^{\infty}\left(\Omega^{\prime}\right)$, então $\chi \mathrm{f} \in \mathrm{H}_{\mathrm{loc}}^{\mathrm{r}, \mathrm{s}}\left(\Omega^{\prime}\right)$.

Demonstração: Seja $\Omega^{\prime \prime} \subseteq \Omega^{\prime}$ aberto. Por hipótese existe $g \in \mathrm{H}^{\mathrm{r}, \mathrm{s}}$ tal que $f=\mathrm{g}$ em $\Omega^{\prime \prime}$. Seja $\varphi \in C_{c}^{\infty}\left(\mathbb{R}^{m+1}\right)$ tal que $\varphi \equiv 1$ em $\Omega^{\prime \prime}$. Pelo lema 2.29, temos que $(\varphi \chi) g \in \mathrm{H}^{r, s}$. Como $\chi f$ coincide com $\chi g=(\varphi \chi) g$ em $\Omega^{\prime \prime}$, o corolário 2.32 permite afirmar que $\chi f \in H^{r, s}$.

Corolário 2.34. Seja $\Omega^{\prime} \subset \subset \mathbb{R}^{m+1}$ aberto. Se $\mathrm{r}, \mathrm{s} \geq 0$, então $\mathrm{H}_{\mathrm{loc}}^{\mathrm{r}+\mathrm{s}}\left(\Omega^{\prime}\right) \subseteq \mathrm{H}_{\mathrm{loc}}^{\mathrm{r}, \mathrm{s}}\left(\Omega^{\prime}\right)$. Se $p \in \mathbb{R}$, existem $r, s \in \mathbb{R}$ tais que $\mathrm{H}_{\mathrm{loc}}^{\mathrm{p}}\left(\Omega^{\prime}\right) \subseteq \mathrm{H}_{\mathrm{loc}}^{\mathrm{r}, \mathrm{s}}\left(\Omega^{\prime}\right)$.

Demonstração: Segue do corolário 2.32 juntamente com as proposições 2.24 e 2.25.

Corolário 2.35. Seja $\Omega^{\prime} \subseteq \mathbb{R}^{\mathrm{m}+1}$ aberto. Então $\mathrm{C}^{\infty}\left(\Omega^{\prime}\right) \subseteq \mathrm{H}_{\mathrm{loc}}^{\mathrm{r}, \mathrm{s}}\left(\Omega^{\prime}\right)$ para quaisquer $r, s \in \mathbb{R}$.

Demonstração: Sejam $r, s \in \mathbb{R}$ dados. Como queremos provar que $C^{\infty}\left(\Omega^{\prime}\right) \subseteq H_{\text {loc }}^{r, s}\left(\Omega^{\prime}\right)$, podemos supor sem perda de generalidade que $r, s \geq 0$. Mas então temos $C^{\infty}\left(\Omega^{\prime}\right) \subseteq$ $\mathrm{H}_{\mathrm{loc}}^{\mathrm{r}+\mathrm{s}} \subseteq \mathrm{H}_{\mathrm{loc}}^{\mathrm{r}, \mathrm{s}}$, pelo corolário anterior e porque

$$
\bigcap_{p \in \mathbb{R}} H_{10 c}^{p}\left(\Omega^{\prime}\right)=C^{\infty}\left(\Omega^{\prime}\right)
$$

Outra propriedade dos espaços de Sobolev parciais que é análoga à dos usuais é enunciada no próximo lema: um operador de derivada agindo em $\mathrm{H}_{\text {loc }}^{\mathrm{r}, \mathrm{s}}$ tem imagem num espaço $\mathrm{H}_{\mathrm{loc}}^{\mathrm{r}^{\prime}, \mathrm{s}^{\prime}}$ maior, fazendo com que os índices percam exatamente a ordem desse operador em cada variável.

Lema 2.36. Sejam $r, s \in \mathbb{R}, \alpha \in \mathbb{N}^{m}, j \in \mathbb{N} e \Omega^{\prime} \subseteq \mathbb{R}^{m+1}$ aberto. Então:

$$
f \in \mathrm{H}_{\mathrm{loc}}^{\mathrm{r}, \mathrm{s}}\left(\Omega^{\prime}\right) \Rightarrow \frac{\partial^{|\alpha|+j}}{\partial \mathrm{t}^{\alpha} \partial x^{j}} f \in \mathrm{H}_{\mathrm{loc}}^{\mathrm{r}-|\alpha|, \mathrm{s}-\mathrm{j}}\left(\Omega^{\prime}\right)
$$


Demonstração: Devido ao corolário 2.32, será suficiente assumir que $f \in \mathrm{H}^{r, s}$ e provar o resultado sem loc. Uma propriedade fundamental da transformada de Fourier diz que

$$
\left(\frac{\partial^{|\alpha|+j}}{\partial t^{\alpha} \partial x^{j}} f\right)^{\wedge}(\xi, \eta)=\xi^{\alpha} \eta^{j} \hat{f}(\xi, \eta)
$$

Portanto,

$$
\begin{aligned}
\left\|\frac{\partial^{|\alpha|+j}}{\partial t^{\alpha} \partial x^{j}} f\right\|_{H^{r-|\alpha|, s-j}}^{2} & =\iint\left(1+|\xi|^{2}\right)^{r-|\alpha|}\left(1+\eta^{2}\right)^{s-j}\left|\left(\frac{\partial^{|\alpha|+j}}{\partial t^{\alpha} \partial x^{j}} f\right)^{\wedge}(\xi, \eta)\right|^{2} d \xi d \eta \\
& =\iint\left(1+|\xi|^{2}\right)^{r-|\alpha|}|\xi|^{2|\alpha|}\left(1+\eta^{2}\right)^{s-j} \eta^{2 j}|\hat{f}(\xi, \eta)|^{2} d \xi d \eta \\
& \leq \iint\left(1+|\xi|^{2}\right)^{r-|\alpha|}\left(1+|\xi|^{2}\right)^{|\alpha|}\left(1+\eta^{2}\right)^{s-j}\left(1+\eta^{2}\right)^{j}|\hat{f}(\xi, \eta)|^{2} d \xi d \eta \\
& =\|f\|_{H^{r, s}}^{2}<\infty
\end{aligned}
$$

Recordemos a chamada regularidade local para operadores elípticos com relação a espaços de Sobolev usuais: se $\mathrm{P}$ é um operador diferencial parcial linear elíptico com coeficientes em $C^{\infty}\left(\Omega^{\prime}\right)$ e de ordem $M$, então vale a implicação

$$
\text { Pf } \in \mathrm{H}_{\mathrm{loc}}^{\mathrm{p}}\left(\Omega^{\prime}\right) \Rightarrow \mathrm{f} \in \mathrm{H}_{\mathrm{loc}}^{\mathrm{p}+\mathrm{M}}\left(\Omega^{\prime}\right)
$$

para todo $p \in \mathbb{R}$ e toda $f \in \mathcal{D}^{\prime}\left(\Omega^{\prime}\right)$. Isto é, pelo fato do operador ser elíptico, podemos recuperar a mesma quantidade de índices de Sobolev que são perdidos quando o operador é aplicado a uma distribuição em $\mathrm{H}_{\text {loc }}^{\mathrm{p}+\mathrm{M}}$. Uma demonstração deste fato pode ser encontrada em [F1], teorema 6.33, p. 214. Seguindo-se as mesmas ideias dessa demonstração, poderíamos provar o resultado análogo para espaços de Sobolev parciais, com relação ao ganho de índices correspondentes à ordem do operador em cada conjunto de variáveis ( $\mathrm{t}$ e x). Essa propriedade teria sido útil em alguns pontos desta seção; entretanto, sua demonstração teria tomado muito tempo do projeto, de forma que optamos por tentar contornar sua utilização em resultados como o da próxima proposição:

Proposição 2.37. Seja $\Omega^{\prime} \subseteq \mathbb{R}^{m+1}$ aberto. Se $f \in \mathcal{D}^{\prime}\left(\Omega^{\prime}\right) e$

$$
(\mathrm{id}-\Delta) f=f-\sum_{j=1}^{m} \frac{\partial^{2}}{\partial t_{j}^{2}} f-\frac{\partial^{2}}{\partial x^{2}} f \in H_{l o c}^{r, s}\left(\Omega^{\prime}\right)
$$

então $f \in \mathrm{H}_{\mathrm{loc}}^{\mathrm{r}+2, \mathrm{~s}}\left(\Omega^{\prime}\right)$.

A propriedade de regularidade local de operadores elípticos provaria ainda mais: $f \in$ $\mathrm{H}_{\mathrm{loc}}^{\mathrm{r}+2, s+2}\left(\Omega^{\prime}\right)$. Ao invés disso, foi possível provar a proposição 2.37 na forma como está apresentada, que, apesar de mais fraca, já nos será suficiente. O que nos possibilita fazê-lo 
é o fato que o operador (id $-\Delta$ ) se comporta bem com relação à transformada de Fourier e aos pesos $\left(1+|\xi|^{2}\right)^{r}$ e $\left(1+\eta^{2}\right)^{s}$ que surgem na definição dos espaços de Sobolev parciais, isto é,

$$
((\mathrm{id}-\Delta) f)^{\wedge}(\xi, \eta)=\left(1+|(\xi, \eta)|^{2}\right) \hat{f}(\xi, \eta)
$$

Demonstração de (2.37). Seja $\Omega_{1} \subset \subset \Omega^{\prime}$ aberto. De acordo com o corolário 2.32, devemos provar que existe $\mathrm{g} \in \mathrm{H}^{\mathrm{r}+2, \mathrm{~s}}$ tal que $\mathrm{f}=\mathrm{g}$ em $\Omega_{1}$. Fixe também um aberto $\Omega_{2}$ tal que $\Omega_{1} \subset \subset \Omega_{2} \subset \subset \Omega^{\prime}$.

Por hipótese e pelo corolário 2.32, existe $w \in \mathrm{H}^{\mathrm{r}, \mathrm{s}}$ tal que $(\mathrm{id}-\Delta) \mathrm{f}=w$ em $\Omega_{2}$. Tome uma função de corte $\chi \in C_{c}^{\infty}\left(\mathbb{R}^{m+1}\right)$ tal que $\chi \equiv 1$ em $\Omega_{1}$. Como $\chi w \in \mathcal{E}^{\prime}\left(\mathbb{R}^{m+1}\right)$, existem $M \in \mathbb{N}, M>0$, e uma distribuição $z \in \mathcal{D}^{\prime}\left(\mathbb{R}^{m+1}\right)$ tais que

$$
\chi w=(\mathrm{id}-\Delta)^{\mathrm{M}} z \quad \text { em } \Omega_{1}
$$

Defina $v=(\mathrm{id}-\Delta)^{M-1} z$. Temos $(\mathrm{id}-\Delta) v=(\mathrm{id}-\Delta)^{M} z=\chi w \in \mathrm{H}^{\mathrm{r}, \mathrm{s}}$, e assim

$$
\begin{aligned}
\|v\|_{H^{r+2, s}}^{2} & =\iint\left(1+|\xi|^{2}\right)^{r+2}\left(1+\eta^{2}\right)^{s}|\hat{v}(\xi, \eta)|^{2} \mathrm{~d} \xi \mathrm{d} \eta \\
& =\iint\left(1+|\xi|^{2}\right)^{\mathrm{r}}\left(1+\eta^{2}\right)^{s}\left(1+|\xi|^{2}\right)^{2}|\hat{v}(\xi, \eta)|^{2} \mathrm{~d} \xi \mathrm{d} \eta \\
& \leq \iint\left(1+|\xi|^{2}\right)^{\mathrm{r}}\left(1+\eta^{2}\right)^{s}\left(1+|\xi|^{2}+\eta^{2}\right)^{2}|\hat{v}(\xi, \eta)|^{2} \mathrm{~d} \xi \mathrm{d} \eta \\
& =\iint\left(1+|\xi|^{2}\right)^{\mathrm{r}}\left(1+\eta^{2}\right)^{s}\left|\left(1+|(\xi, \eta)|^{2}\right) \hat{v}(\xi, \eta)\right|^{2} \mathrm{~d} \xi \mathrm{d} \eta \\
& =\iint\left(1+|\xi|^{2}\right)^{\mathrm{r}}\left(1+\eta^{2}\right)^{s}\left|((\mathrm{id}-\Delta) v)^{\wedge}(\xi, \eta)\right|^{2} \mathrm{~d} \xi \mathrm{d} \eta<\infty
\end{aligned}
$$

isto é, $v \in \mathrm{H}^{\mathrm{r}+2, \mathrm{~s}}$. Note ainda que, em $\Omega_{1}$, temos

$$
(\mathrm{id}-\Delta) v=\chi w=w=(\mathrm{id}-\Delta) \mathrm{f} \Rightarrow(\mathrm{id}-\Delta)(\mathrm{f}-v)=0
$$

Dessa forma $f-v \in \mathcal{D}^{\prime}\left(\Omega_{1}\right)$ pertence ao núcleo de id $-\Delta$, que está contido em $C^{\infty}\left(\Omega_{1}\right)$, uma vez que id $-\Delta$ é hipoelíptico.

Agora fixe $\tau \in C_{c}^{\infty}\left(\mathbb{R}^{m+1}\right)$ tal que $\tau \equiv 1$ em $\Omega_{1}$. Temos que $\tau(f-v) \in C^{\infty}\left(\mathbb{R}^{m+1}\right) \subseteq$ $H^{r+2, s}$. Logo, $g=v+\tau(f-v)$ é uma distribuição de $H^{r+2, s}$ que coincide $\operatorname{com} v+1(f-v)=f$ em $\Omega_{1}$, como queríamos.

Já podemos voltar nosso foco ao estudo local das soluções da estrutura $\mathbb{L}$. Então suponha a partir daqui que $\Omega$ e $\mathbb{L}$ são como no início da seção 2.1 , isto é, que $\Omega$ é um aberto de $\mathbb{R}^{m+1}=\left\{\left(t_{1}, \ldots, t_{m}, x\right)\right\}$ e que a estrutura $\mathbb{L}$ é gerada pelos campos

$$
L_{j}=\frac{\partial}{\partial t_{j}}+\lambda_{j}(t, x) \frac{\partial}{\partial x} \quad, \quad j=1, \ldots, m
$$


onde $\lambda_{j}$ são funções analíticas. Recordamos que existe em $\Omega$ uma integral primeira para $\mathbb{L}$,

$$
z(t, x)=x+i \phi(t, x)
$$

onde $\phi$ é analítica a valores reais e satisfaz $\phi(0, x)=0$ para todo $x$. Finalmente, também havíamos definido o campo $\mathrm{L}_{0}=\left(1 / z_{x}\right) \partial_{x}$.

Lema 2.38. (Fórmula de Leibniz Transposta) Seja $\vee \subseteq \mathbb{R}$ aberto. Para todo natural $\mathrm{M}$, toda distribuição $\mathrm{g} \in \mathcal{D}^{\prime}(\mathrm{V})$ e toda função $\mathrm{f} \in \mathrm{C}^{\infty}(\mathrm{V})$, vale a fórmula

$$
f \frac{d^{M} g}{d \tau^{M}}=\sum_{j=0}^{M}(-1)^{j}\left(\begin{array}{c}
M \\
j
\end{array}\right) \frac{d^{M-j}}{d \tau^{M-j}}\left(\frac{d^{j} f}{d \tau^{j}} g\right)
$$

Demonstração: Seja $\phi=\phi(\tau)$ uma função teste. Denotemos por $\mathfrak{u}^{(j)}$ a derivada de ordem j de uma função ou distribuição u da variável real $\tau$. Pela Regra de Leibniz usual:

$$
\begin{aligned}
\left\langle f g^{(M)}, \phi\right\rangle & =\left\langle g^{(M)}, f \phi\right\rangle=(-1)^{M}\left\langle g,(f \phi)^{(M)}\right\rangle=(-1)^{M}\left\langle g, \sum_{j=0}^{M}\left(\begin{array}{c}
M \\
j
\end{array}\right) f^{(j)} \phi^{(M-j)}\right\rangle= \\
& =\sum_{j=0}^{M}(-1)^{M}\left(\begin{array}{c}
M \\
j
\end{array}\right)\left\langle f^{(j)} g, \phi^{(M-j)}\right\rangle=\sum_{j=0}^{M}(-1)^{M}\left(\begin{array}{c}
M \\
j
\end{array}\right)(-1)^{M-j}\left\langle\left(f^{(j)} g\right)^{(M-j)}, \phi\right\rangle= \\
& =\left\langle\sum_{j=0}^{M}(-1)^{2 M-j}\left(\begin{array}{c}
M \\
j
\end{array}\right)\left(f^{(j)} g\right)^{(M-j)}, \phi\right\rangle
\end{aligned}
$$

Basta agora notarmos que $(-1)^{2 M-j}=(-1)^{j}$.

Proposição 2.39. Sejam $\mathrm{U} \subseteq \Omega$ aberto, $\mathrm{u} \in \mathcal{D}^{\prime}(\mathrm{U})$ e $\mathrm{N} \in \mathbb{N}$. Então $u=\mathrm{L}_{0}^{\mathrm{N}} v_{1}$ para alguma $v_{1} \in \mathrm{C}^{0}(\mathrm{U})$ se e somente se $\mathrm{u}=\partial_{\chi}^{\mathrm{N}} v_{2}$ para alguma $v_{2} \in \mathrm{C}^{0}(\mathrm{U})$.

Demonstração: Ambas as implicações são provadas por indução em $\mathrm{N}$, sendo que o caso $\mathrm{N}=0$ é trivial: basta tomar $v_{1}=v_{2}$. Para provarmos a primeira implicação, suponha que $u=\mathrm{L}_{0}^{\mathrm{N}+1} v_{1}$ para alguma $v_{1}$ contínua, e que toda distribuição na forma $\mathrm{L}_{0}^{\mathrm{N}} \mathrm{f}_{1}$ ( $\mathrm{f}_{1}$ contínua) se escreve como $\partial_{x}^{N_{f}} f_{2}\left(f_{2}\right.$ contínua); essa é a hipótese de indução. Em particular, existe $w$ contínua tal que $\mathrm{L}_{0}^{\mathrm{N}} v_{1}=\partial_{x}^{\mathrm{N}} \mathcal{w}$, e temos

$$
\mathrm{u}=\mathrm{L}_{0}^{\mathrm{N}+1} v_{1}=\mathrm{L}_{0}\left(\mathrm{~L}_{0}^{\mathrm{N}} v_{1}\right)=\frac{1}{z_{x}} \frac{\partial}{\partial x}\left(\frac{\partial^{\mathrm{N}} \mathcal{w}}{\partial x^{\mathrm{N}}}\right)=\frac{1}{z_{x}} \frac{\partial^{\mathrm{N}+1} \mathcal{w}}{\partial x^{\mathrm{N}+1}}
$$

Defina a função contínua

$$
v_{2}=\sum_{j=0}^{N+1}(-1)^{j}\left(\begin{array}{c}
N+1 \\
j
\end{array}\right)\left(\frac{\partial}{\partial x}\right)^{-j}\left(\frac{\partial^{j}\left(1 / z_{x}\right)}{\partial x^{j}} w\right)
$$


onde a notação $\left(\partial_{x}\right)^{-j} g$ significa uma primitiva de ordem $j$ da distribuição $g$ com relação a $x$, que existe porque se trata de uma única variável real. Pela Fórmula de Leibniz Transposta, temos $\left(\partial_{\chi}\right)^{\mathrm{N}+1} v_{2}=\left(1 / z_{\chi}\right)\left(\partial_{\chi}\right)^{N+1} w=u$, como queríamos.

Reciprocamente, suponha que $u=\partial_{x}^{N+1} v_{2}$ para alguma $v_{2}$ contínua. Seja a função contínua

$$
w=\sum_{j=0}^{N+1}(-1)^{j}\left(\begin{array}{c}
N+1 \\
j
\end{array}\right)\left(\frac{\partial}{\partial x}\right)^{-j}\left(\frac{\partial^{j} z_{x}}{\partial x^{j}} v_{2}\right)
$$

A Fórmula de Leibniz Transposta implica que $\partial_{\chi}^{\mathrm{N}+1} \mathcal{W}=z_{\chi} \partial_{\chi}^{\mathrm{N}+1} v_{2}=z_{\chi} u$, e por hipótese de indução existe $v_{1}$ contínua tal que $\partial^{\mathrm{N}} \mathcal{W}=\mathrm{L}_{0}^{\mathrm{N}} v_{1}$. Segue que

$$
z_{x} u=\frac{\partial}{\partial x} \frac{\partial^{N} \mathcal{w}}{\partial x^{N}}=\frac{\partial}{\partial x} \mathrm{~L}_{0}^{\mathrm{N}} v_{1} \Rightarrow u=\frac{1}{z_{x}} \frac{\partial}{\partial x} \mathrm{~L}_{0}^{\mathrm{N}} v_{1}=\mathrm{L}_{0}^{\mathrm{N}+1} v_{1}
$$

O resultado chave que combina espaços de Sobolev e os operadores $L_{j}$ é a próxima proposição. Ela afirma que soluções locais $u$ de $\mathbb{L}$ pertencem a espaços $H_{\text {loc }}^{\text {rss }}$ com $r$ arbitrariamente grande, mas em geral com $s$ muito negativo. A ideia da prova é usar os operadores $\mathrm{L}_{j}$ para trocar derivadas de $u$ com relação $a t_{j}$ pelas derivadas com relação a $x$.

Proposição 2.40. Seja $\Omega^{\prime} \subset \subset \Omega$ aberto. Se $u \in \mathcal{D}^{\prime}(\Omega)$ é solução de $\mathbb{L}$ em $\Omega^{\prime}$, então para todo $\mathrm{r} \in \mathbb{R}$ existe $\mathrm{s} \in \mathbb{R}$ tal que $\mathrm{u} \in \mathrm{H}_{\mathrm{loc}}^{\mathrm{r}, \mathrm{s}}\left(\Omega^{\prime}\right)$.

Demonstração: Como $\Omega^{\prime} \subset \subset \Omega$, temos que a restrição $\left.u\right|_{\Omega^{\prime}}$, que denotaremos também por $u$, pertence a $H_{\text {loc }}^{p}\left(\Omega^{\prime}\right)$ para algum $p \in \mathbb{R}$, e o corolário 2.34 nos dá que $u \in H_{\text {loc }}^{a, b}\left(\Omega^{\prime}\right)$ para algum par $a, b$ de números reais. Se $a \geq r$, terminamos. Então suponha que $a<r$.

$\operatorname{Em} \Omega^{\prime}$, valem por hipótese as m equações:

$$
\frac{\partial u}{\partial t_{j}}(t, x)=-\lambda_{j}(t, x) \frac{\partial u}{\partial x}(t, x), \quad j=1, \ldots, m
$$

Logo,

$$
\begin{aligned}
(\mathrm{id}-\Delta) u & =u-\sum_{j=1}^{m} \frac{\partial^{2} u}{\partial t_{j}^{2}}-\frac{\partial^{2} u}{\partial x^{2}} \\
& =u-\sum_{j=1}^{m} \frac{\partial}{\partial t_{j}}\left(-\lambda_{j} \frac{\partial u}{\partial x}\right)-\frac{\partial^{2} u}{\partial x^{2}} \\
& =u+\sum_{j=1}^{m}\left(\frac{\partial \lambda_{j}}{\partial t_{j}} \frac{\partial u}{\partial x}+\lambda_{j} \frac{\partial^{2} u}{\partial t_{j} \partial x}\right)-\frac{\partial^{2} u}{\partial x^{2}}
\end{aligned}
$$

A primeira parcela dessa soma pertence a $H_{\text {loc }}^{\mathrm{a}, \mathrm{b}}\left(\Omega^{\prime}\right)$. O somatório está em $\mathrm{H}_{\mathrm{loc}}^{\mathrm{a}, \mathrm{b}-1}\left(\Omega^{\prime}\right)+$ $\mathrm{H}_{\mathrm{loc}}^{\mathrm{a}-1, \mathrm{~b}-1}\left(\Omega^{\prime}\right) \subseteq \mathrm{H}_{\mathrm{loc}}^{\mathrm{a}-1, \mathrm{~b}-1}\left(\Omega^{\prime}\right)$, de acordo com o lema 2.36 e o corolário 2.33. E a última parcela pertence a $\mathrm{H}_{\mathrm{loc}}^{\mathrm{a}-1, \mathrm{~b}-2}\left(\Omega^{\prime}\right)$ pelo lema 2.36. Assim temos que

$$
(\mathrm{id}-\Delta) u \in \mathrm{H}_{\mathrm{loc}}^{\mathrm{a}, \mathrm{b}}\left(\Omega^{\prime}\right)+\mathrm{H}_{\mathrm{loc}}^{\mathrm{a}-1, \mathrm{~b}-1}\left(\Omega^{\prime}\right)+\mathrm{H}_{\mathrm{loc}}^{\mathrm{a}-1, \mathrm{~b}-2}\left(\Omega^{\prime}\right) \subseteq \mathrm{H}_{\mathrm{loc}}^{\mathrm{a}-1, \mathrm{~b}-2}\left(\Omega^{\prime}\right)
$$


e a proposição 2.37 implica que $u \in \mathrm{H}_{\mathrm{loc}}^{\mathrm{a}+1, \mathrm{~b}-2}\left(\Omega^{\prime}\right)$. Agora aplique este mesmo cálculo mais algumas vezes, substituindo $a, b$ no início por $a+1, b-2$. Obtemos sucessivamente que

$$
u \in \mathrm{H}_{\mathrm{loc}}^{\mathrm{a}+2, \mathrm{~b}-4}\left(\Omega^{\prime}\right) \quad, \quad u \in \mathrm{H}_{\mathrm{loc}}^{\mathrm{a}+3, \mathrm{~b}-6}\left(\Omega^{\prime}\right) \quad, \quad u \in \mathrm{H}_{\mathrm{loc}}^{\mathrm{a}+4, \mathrm{~b}-8}\left(\Omega^{\prime}\right) \quad, \quad \text { etc. }
$$

Ou seja, após $r-a$ aplicações do mesmo cálculo, concluímos que $u \in H_{l o c}^{r, b-2(r-a)}\left(\Omega^{\prime}\right)$.

Teorema 2.41. Se $u \in \mathcal{D}^{\prime}(\Omega)$ e $\mathbb{L} u=0$, então para todo aberto $\Omega^{\prime} \subset \subset \Omega$ existem $\mathrm{N} \in \mathbb{N}$ e $\mathrm{G} \in \mathrm{C}^{0}\left(\Omega^{\prime}\right)$ tais que $\mathrm{u}=\frac{\partial^{\mathrm{N}} \mathrm{G}}{\partial x^{\mathrm{N}}}$ como distribuições em $\Omega^{\prime}$.

Demonstração: Podemos supor que $\Omega^{\prime}=\mathcal{B}_{r^{\prime}} \times J^{\prime}$ para alguma bola aberta $\mathcal{B}_{r^{\prime}} \subseteq \mathbb{R}^{m}$ e algum intervalo aberto $\mathrm{J}^{\prime} \subseteq \mathbb{R}$. Seja $r>0$ tal que

$$
\int_{\mathbb{R}^{m}}\left(1+|\xi|^{2}\right)^{-r} d \xi<\infty
$$

Pela proposição anterior, $u \in \mathrm{H}_{\mathrm{loc}}^{r, s}\left(\Omega^{\prime}\right)$ para algum $s \in \mathbb{R}$. Em particular, $u$ coincide em $\Omega^{\prime}$ com uma distribuição $U \in H^{r, s}$.

Para cada $\psi \in \mathcal{S}\left(\mathrm{J}^{\prime}\right)$, defina a função $\mathrm{f}_{\psi}: \mathcal{B}_{\mathrm{r}^{\prime}} \rightarrow \mathbb{C}$ por

$$
f_{\psi}(t)=\frac{1}{(2 \pi)^{m}} \int_{\mathbb{R}} \int_{\mathbb{R}^{m}} \widehat{u}(\xi, \eta) e^{i \xi t} \check{\psi}(\eta) d \xi d \eta
$$

Temos que verificar que $f_{\psi}(t)$ está bem-definida. De fato, pela Desigualdade de Hölder,

$$
\begin{aligned}
(2 \pi)^{\mathrm{m}}\left|\mathrm{f}_{\psi}(\mathrm{t})\right| & \leq \iint|\hat{\mathrm{U}}(\xi, \eta) \| \breve{\psi}(\eta)| \mathrm{d} \xi \mathrm{d} \eta \\
& =\iint\left(1+|\xi|^{2}\right)^{r / 2}\left(1+\eta^{2}\right)^{s / 2}|\widehat{\mathrm{U}}(\xi, \eta)| \cdot \\
& \cdot\left(1+|\xi|^{2}\right)^{-r / 2}\left(1+\eta^{2}\right)^{-s / 2}|\check{\psi}(\eta)| \mathrm{d} \xi \mathrm{d} \eta \\
& \leq\left(\iint\left(1+|\xi|^{2}\right)^{r}\left(1+\eta^{2}\right)^{s}|\widehat{\mathrm{U}}(\xi, \eta)|^{2} \mathrm{~d} \xi \mathrm{d} \eta\right)^{1 / 2} \cdot \\
& =\|\mathrm{U}\|_{H^{r, s}}\left(\int\left(1+|\xi|^{2}\right)^{-r} \mathrm{~d} \xi\right)^{1 / 2}\left(\int\left(1+\eta^{2}\right)^{-s}|\check{\psi}(\eta)|^{2} \mathrm{~d} \eta\right)^{1 / 2} \\
& =\|\mathrm{U}\|_{H^{r, s}}\left(\int\left(1+|\xi|^{2}\right)^{-r} \mathrm{~d} \xi\right)^{1 / 2}\left\|(\check{\psi})^{-}\right\|_{H^{-s}}<\infty
\end{aligned}
$$


Note também que a mesma conta prova que $f_{\psi}$ é contínua em $\mathcal{B}_{r^{\prime}}$, pelo teorema $d a$ convergência dominada. Ainda, dada $\phi \in \mathrm{C}_{\mathrm{c}}^{\infty}\left(\mathcal{B}_{\mathrm{r}^{\prime}}\right)$, temos

$$
\begin{aligned}
\int f_{\psi}(t) \phi(t) d t & =\frac{1}{(2 \pi)^{m}} \iiint \widehat{u}(\xi, \eta) e^{i \xi t} \breve{\psi}(\eta) d \xi d \eta \phi(t) d t \\
& =\iint \widehat{u}(\xi, \eta)\left(\frac{1}{(2 \pi)^{m}} \int e^{i \xi t} \phi(t) d t\right) \check{\psi}(\eta) d \xi d \eta \\
& =\iint \widehat{u}(\xi, \eta) \check{\phi}(\xi) \check{\psi}(\eta) d \xi d \eta \\
& =\iint \widehat{u}(\xi, \eta)(\phi \otimes \psi)^{\vee}(\xi, \eta) d \xi d \eta \\
& =\left\langle\widehat{u},(\phi \otimes \psi)^{\sim}\right\rangle=\langle u, \phi \otimes \psi\rangle
\end{aligned}
$$

e o que justifica mudarmos a ordem de integração é o Teorema de Fubini, já que o integrando tem norma $\mathrm{L}^{1}$ finita. Defina agora $\mathrm{g}: \mathcal{B}_{\mathrm{r}^{\prime}} \times \mathrm{J}^{\prime} \rightarrow \mathbb{C}$ por

$$
g(t, x)=\int_{\mathbb{R}^{\prime}} \int_{\mathbb{R}^{m}}\left(1+\eta^{2}\right)^{-M} e^{i \eta x} e^{i \xi t} \hat{u}(\xi, \eta) d \xi d \eta
$$

onde $M \geq 0$ é escolhido de forma que $\left(1+\eta^{2}\right)^{-2 M-s}$ seja integrável em $\mathbb{R}$. Novamente pelo teorema da convergência dominada, temos g contínua:

$$
\begin{aligned}
|g(t, x)| & \leq \iint|\hat{\mathrm{U}}(\xi, \eta)|\left(1+\eta^{2}\right)^{-\mathrm{M}} \mathrm{d} \xi \mathrm{d} \eta \\
& =\iint\left(1+|\xi|^{2}\right)^{\mathrm{r} / 2}\left(1+\eta^{2}\right)^{s / 2}|\widehat{\mathrm{U}}(\xi, \eta)|\left(1+|\xi|^{2}\right)^{-\mathrm{r} / 2}\left(1+\eta^{2}\right)^{-s / 2-M} \mathrm{~d} \xi \mathrm{d} \eta \\
& \leq\left(\iint\left(1+|\xi|^{2}\right)^{\mathrm{r}}\left(1+\eta^{2}\right)^{s}|\widehat{\mathrm{U}}(\xi, \eta)|^{2} \mathrm{~d} \xi \mathrm{d} \eta\right)^{1 / 2} \cdot \\
& \cdot\left(\iint\left(1+|\xi|^{2}\right)^{-\mathrm{r}}\left(1+\eta^{2}\right)^{-2 M-s} \mathrm{~d} \xi \mathrm{d} \eta\right)^{1 / 2} \\
& =\|\mathrm{U}\|_{\mathrm{H}^{r, s}}\left(\int\left(1+|\xi|^{2}\right)^{-r} \mathrm{~d} \xi\right)^{1 / 2}\left(\int\left(1+\eta^{2}\right)^{-2 M-s} \mathrm{~d} \eta\right)^{1 / 2}<\infty
\end{aligned}
$$

Finalmente, para cada $t \in \mathcal{B}_{\mathrm{r}^{\prime}}$, seja $\mathcal{w}_{\mathrm{t}}: \mathcal{S}(\mathbb{R}) \rightarrow \mathbb{C}$ a distribuição temperada

$$
\left\langle w_{\mathrm{t}}, \psi\right\rangle=\int_{\mathbb{R}^{\prime}} \int_{\mathbb{R}^{m}}\left(1+\eta^{2}\right)^{-\mathrm{M}} e^{\mathrm{i} \xi \mathrm{t}} \widehat{\mathrm{U}}(\xi, \eta) \psi(\eta) \mathrm{d} \xi \mathrm{d} \eta \quad, \quad \psi \in \mathcal{S}(\mathbb{R})
$$

O mesmo tipo de estimativa que fizemos em (2.42) prova que $w_{t}$ está bem definida e é de fato temperada. Agora, se $\mathcal{F}_{x} g(t, \cdot)$ denota a transformada de Fourier parcial com relação 
a $x$ da função $g(t, x)$, com $t$ fixo, então, para toda $\psi \in \mathcal{S}(\mathbb{R})$,

$$
\begin{aligned}
\left\langle\mathcal{F}_{x} g(t, \cdot), \psi\right\rangle & =\langle g(t, \cdot), \hat{\psi}\rangle \\
& =\iiint\left(1+\eta^{2}\right)^{-M} e^{i \eta x} e^{i \xi t} \widehat{u}(\xi, \eta) d \xi d \eta \hat{\psi}(x) d x \\
& =2 \pi \iint\left(1+\eta^{2}\right)^{-M} e^{i \xi t} \hat{u}(\xi, \eta) d \xi d \eta \psi(\eta) \\
& =2 \pi\left\langle w_{t}, \psi\right\rangle
\end{aligned}
$$

isto é, $\mathcal{F}_{x} g(t, \cdot)=2 \pi w_{t}$. Em particular,

$$
\begin{aligned}
\left\langle\mathcal{F}_{x}\left(\mathrm{id}-\frac{\partial^{2}}{\partial x^{2}}\right)^{M} g(t, \cdot), \psi\right\rangle & =\left\langle\left(1+\eta^{2}\right)^{M} \mathcal{F}_{x} g(t, \eta), \psi(\eta)\right\rangle \\
& =\left\langle\mathcal{F}_{x} g(t, \eta),\left(1+\eta^{2}\right)^{M} \psi(\eta)\right\rangle \\
& =2 \pi\left\langle w_{t}(\eta),\left(1+\eta^{2}\right)^{M} \psi(\eta)\right\rangle \\
& =2 \pi \iint\left(1+\eta^{2}\right)^{-M} e^{i \xi t} \hat{u}(\xi, \eta)\left(1+\eta^{2}\right)^{M} \psi(\eta) d \xi d \eta \\
& =2 \pi \iint e^{i \xi \xi} \hat{u}(\xi, \eta) \psi(\eta) d \xi d \eta \\
& =(2 \pi)^{m+1} f_{\hat{\psi}}(t)
\end{aligned}
$$

Mas note que qualquer soma de derivadas de diversas ordens com relação a $x$ de funções contínuas pode ser escrita como uma única derivada de ordem alta com relação a $x$ de uma função contínua. De fato, se uma distribuição $f \in \mathcal{D}^{\prime}\left(\mathcal{B}_{r^{\prime}} \times J^{\prime}\right)$ é dada na forma

$$
f=\sum_{j=0}^{p} \frac{\partial^{j} h_{j}}{\partial x^{j}}
$$

onde cada $h_{j}=h_{j}(t, x)$ é contínua em $\mathcal{B}_{r^{\prime}} \times J^{\prime}$, e se denotarmos por $h_{j}^{(-k)}$ uma função (de classe $C^{k}$ ) tal que

$$
\frac{\partial^{k} h_{j}^{(-k)}}{\partial x^{k}}=h_{j} \quad, \quad j=1, \ldots, p \quad, \quad k \in \mathbb{N}
$$

então temos $f=\frac{\partial^{p}}{\partial x^{p}}\left(\sum_{j=0}^{p} h_{j}^{-p+j}\right)$. Dessa forma, existe $G=G(t, x)$ contínua satisfazendo

$$
\left(\mathrm{id}-\frac{\partial^{2}}{\partial x^{2}}\right)^{M} g=\frac{\partial^{N} G}{\partial x^{N}}
$$


(onde $N=2 M$ ). Portanto, para quaisquer $\phi \in \mathrm{C}_{\mathrm{C}}^{\infty}\left(\mathcal{B}_{\mathrm{r}^{\prime}}\right)$ e $\psi \in \mathrm{C}_{\mathrm{C}}^{\infty}\left(\mathrm{J}^{\prime}\right)$, as equações (2.43) e (2.44) implicam que

$$
\begin{aligned}
(2 \pi)^{m+1}\langle u, \phi \otimes \psi\rangle & =\int(2 \pi)^{m+1} f_{\psi}(t) \phi(t) d t \\
& =\int\left\langle\mathcal{F}_{x}\left(i d-\frac{\partial^{2}}{\partial x^{2}}\right)^{M} g(t, \cdot), \check{\psi}\right\rangle \phi(t) d t \\
& =\int\left\langle\mathcal{F}_{x}\left(\frac{\partial^{N} G}{\partial x^{N}}\right)(t, \cdot), \check{\psi}\right\rangle \phi(t) d t \\
& =\int\left\langle\frac{\partial^{N} G}{\partial x^{N}}(t, \cdot), \psi\right\rangle \phi(t) d t \\
& =(-1)^{N} \int\left\langle G(t, \cdot), \frac{\partial^{N} \psi}{\partial x^{N}}\right\rangle \phi(t) d t \\
& =(-1)^{N} \iint G(t, x) \frac{\partial^{N} \psi}{\partial x^{N}}(x) \phi(t) d t d x \\
& =(-1)^{N}\left\langle G, \frac{\partial^{N}}{\partial x^{N}}(\phi \otimes \psi)\right\rangle \\
& =\left\langle\frac{\partial^{N} G}{\partial x^{N}}, \phi \otimes \psi\right\rangle
\end{aligned}
$$

Dada a densidade do subespaço vetorial gerado pelos produtos tensoriais $\phi \otimes \psi$ em $\mathrm{C}_{\mathrm{c}}^{\infty}\left(\mathcal{B}_{\mathrm{r}^{\prime}} \times\right.$ $\mathrm{J}^{\prime}$ ), isto prova que

$$
u=\frac{\partial^{N}}{\partial x^{N}}\left(\frac{G}{(2 \pi)^{m+1}}\right) \quad \text { como distribuições em } \mathcal{B}_{r^{\prime}} \times J^{\prime} .
$$

Lema 2.45. Seja $z(t, x)$ a integral primeira do início da seção 2.1. Suponha que a seguinte equação polinomial em z é válida num aberto de $\Omega$ :

$$
c_{0}+z(t, x) c_{1}+\cdots+z(t, x)^{M} c_{M}=0
$$

para certas distribuições $\mathrm{c}_{j} \in \mathcal{D}^{\prime}(\Omega)$ que satisfazem $\partial_{x} c_{j}=0, j=0, \ldots, M$. Então $\mathrm{c}_{\mathrm{j}} \equiv 0$ para todo $\mathrm{j}$.

Demonstração: Façamos indução em $M$. Não há nada para provar se $M=0$, então suponha que o resultado é válido para $M$, e que

$$
c_{0}+z(t, x) c_{1}+\cdots+z(t, x)^{M+1} c_{M+1}=0
$$

onde $\partial_{x} c_{j}=0, j=0, \ldots, M+1$. Derivando (2.46) com relação a $x$, temos

$$
z_{\chi} c_{1}+2 z z_{\chi} c_{2}+3 z^{2} z_{\chi} c_{3}+\ldots+(M+1) z^{M} z_{\chi} c_{M+1}=0
$$


Como $z_{x}$ nunca se anula, isto é equivalente a

$$
c_{1}+2 z c_{2}+3 z^{2} c_{3}+\ldots+(M+1) z^{M} c_{M+1}=0
$$

e por hipótese de indução concluímos que $c_{1} \equiv \cdots \equiv c_{M+1} \equiv 0$, o que implica que (2.46) é equivalente a $c_{0} \equiv 0$.

Finalmente podemos demonstrar o teorema que anunciamos ao início desta seção:

Teorema 2.47. (Baouendi-Trèves) Seja $\omega_{0} \in \Omega$. Se u $\in \mathcal{D}^{\prime}(\Omega)$ e $\mathbb{L} u=0$, então, para qualquer vizinhança aberta $\Omega^{\prime}$ de $\omega_{0}$ suficientemente pequena, existem $N \in \mathbb{N} e$ $w \in \mathrm{C}^{0}\left(\Omega^{\prime}\right)$ tais que

$$
\mathbb{L} w=0 \quad \text { e } \quad \mathrm{u}=\mathrm{L}_{0}^{\mathrm{N}} \mathfrak{w} \quad \text { em } \Omega^{\prime}
$$

Demonstração: Suponha novamente que $\Omega^{\prime}$ tem a forma $\mathcal{B}_{\mathrm{r}^{\prime}} \times \mathrm{J}^{\prime}$, como acima, e que em $\Omega^{\prime}$ valem (2.3) e (2.4), a respeito da forma local de $L_{j}$ e $z$, com $\omega_{0}=0$. É essa suposição que justifica a condição vizinhança suficientemente pequena no enunciado. Sabemos do teorema 2.41 que existem $\mathrm{G} \in \mathrm{C}^{0}\left(\mathcal{B}_{\mathrm{r}^{\prime}} \times \mathrm{J}^{\prime}\right)$ e $\mathrm{N} \in \mathbb{N}$ tais que

$$
u=\frac{\partial^{N} G}{\partial x^{N}} \quad \operatorname{em} \mathcal{B}_{r^{\prime}} \times J^{\prime}
$$

Pela proposição 2.39, temos $u=\mathrm{L}_{0}^{\mathrm{N}} \mathrm{H}$ em $\mathcal{B}_{\mathrm{r}^{\prime}} \times \mathrm{J}^{\prime}$, para alguma $\mathrm{H} \in \mathrm{C}^{0}\left(\mathcal{B}_{\mathrm{r}^{\prime}} \times \mathrm{J}^{\prime}\right)$. Mas não sabemos se $H$ é solução de $\mathbb{L}$, e nosso trabalho será modificá-la de forma que isso seja satisfeito.

Como $L_{0}$ comuta com cada $L_{j}$, temos que $L_{0}^{N}\left(L_{j} H\right)=0, j=1, \ldots, m$. Provemos que isso implica que existem distribuições $c_{j, v} \in \mathcal{D}^{\prime}\left(\mathcal{B}_{r^{\prime}} \times J^{\prime}\right)$ tais que

$$
\partial_{x} c_{j, v}=0 \quad, \quad L_{j} H=\sum_{v=0}^{N-1} z^{\nu} c_{j, v}
$$

Fixe j. Por indução em $N \geq 1$, suponha inicialmente que $L_{0}\left(L_{j} H\right)=0$, isto é,

$$
\frac{1}{z_{x}} \frac{\partial}{\partial x}\left(L_{j} H\right)=0
$$

Portanto $\partial_{x}\left(L_{j} H\right)=0$, e então $c_{j, 0}=L_{j} H$ é uma distribuição que anula o operador $\partial_{x}$, provando a base da indução. Agora suponha que a afirmação vale para um dado $\mathrm{N}$, e que $\mathrm{L}_{0}^{\mathrm{N}+1}\left(\mathrm{~L}_{j} \mathrm{H}\right)=0$. Então $\mathrm{L}_{0}^{\mathrm{N}}\left(\mathrm{L}_{0} \mathrm{~L}_{j} \mathrm{H}\right)=0$, e por hipótese de indução (aplicada a $\mathrm{L}_{0} \mathrm{~L}_{j} \mathrm{H}$ ) temos

$$
\mathrm{L}_{\mathrm{o}} \mathrm{L}_{\mathrm{j}} \mathrm{H}=\sum_{v=0}^{\mathrm{N}-1} z^{v} \mathrm{c}_{\mathrm{j}, v}
$$


para certas distribuições $c_{j, v}$ que anulam $\partial_{x}$. Logo,

$$
\frac{\partial}{\partial x}\left(L_{j} H\right)=\sum_{v=0}^{N-1} z^{v} z_{x} c_{j, v}=\frac{\partial}{\partial x}\left(\sum_{v=1}^{N} z^{\nu} \frac{c_{j, v-1}}{v}\right)
$$

e dessa forma $d_{j, 0}=L_{j} H-\sum_{v=1}^{N} z^{v}\left(c_{j, v-1} / v\right)$ é uma distribuição que anula $\partial_{x}$. Finalmente, é claro que

$$
\mathrm{L}_{\mathrm{j}} \mathrm{H}=\mathrm{d}_{\mathrm{j}, 0}+\sum_{v=1}^{\mathrm{N}} z^{v}\left(\mathrm{c}_{\mathrm{j}, v-1} / v\right)=\sum_{v=0}^{\mathrm{N}} z^{v} \mathrm{~d}_{\mathrm{j}, v} \quad\left(\mathrm{~d}_{\mathrm{j}, v}=\frac{\mathrm{c}_{\mathrm{j}, v-1}}{v}, v \geq 1\right)
$$

provando o passo indutivo.

Obtemos de (2.48) acima que

$$
\begin{aligned}
\mathrm{L}_{k} \mathrm{~L}_{j} \mathrm{H} & =\sum_{\nu=0}^{\mathrm{N}-1} z^{v}\left(\mathrm{~L}_{k} \mathrm{c}_{j, v}\right)+\sum_{v=0}^{\mathrm{N}-1} \mathrm{~L}_{k}\left(z^{v}\right) \mathrm{c}_{j, v} \\
& =\sum_{\nu=0}^{\mathrm{N}-1} z^{v}\left(\frac{\partial c_{j, v}}{\partial t_{k}}+\lambda_{j} \frac{\partial c_{j, v}}{\partial x}\right)+\sum_{v=0}^{N-1}\left(v z^{v-1} \mathrm{~L}_{k} z\right) c_{j, v} \\
& =\sum_{v=0}^{N-1} z_{v} \frac{\partial c_{j, v}}{\partial t_{k}}
\end{aligned}
$$

Do fato que cada $L_{j}$ comuta com cada $L_{k}, j, k=1, \ldots, m$, concluímos que

$$
\sum_{v=0}^{\mathrm{N}-1} z^{\nu} \frac{\partial \mathrm{c}_{j, v}}{\partial t_{k}}=\sum_{\nu=0}^{\mathrm{N}-1} z^{\nu} \frac{\partial \mathrm{c}_{k, v}}{\partial t_{j}} \quad, \quad j, k=1, \ldots, \mathrm{m}
$$

Usando o lema 2.45, segue que

$$
\frac{\partial c_{j, v}}{\partial t_{k}}=\frac{\partial c_{k, v}}{\partial t_{j}} \quad, \quad j, k=1, \ldots, m, v=0, \ldots, N-1
$$

Esta equação implica que, para cada $v$ fixado, a seguinte corrente em $\Omega^{\prime}$ (isto é, forma diferencial com coeficientes distribucionais)

$$
\omega_{v}=c_{1, v} d t_{1}+\cdots+c_{m, v} d t_{m}
$$

é fechada. De fato,

$$
d \omega_{v}=\sum_{j=1}^{m} \sum_{k=1}^{m} \frac{\partial c_{j, v}}{\partial t_{k}} d t_{k} \wedge d t_{j}=2 \sum_{j<k}\left(\frac{\partial c_{j, v}}{\partial t_{k}}-\frac{\partial c_{k, v}}{\partial t_{j}}\right) d t_{j} \wedge d t_{k}=0
$$

Assim como no caso de formas diferenciais ordinárias, isto implica que $\omega$ é exata, dado que $\Omega^{\prime}$ é simplesmente conexo - vide [R], cap. 4. Portanto, para cada $j=1, \ldots, m$, existe 
uma distribuição $C_{v} \in \mathcal{D}^{\prime}\left(\Omega^{\prime}\right)$ satisfazendo $\partial_{t_{j}} C_{v}=c_{j, v}$, o que transforma a equação (2.48) em

$$
\mathrm{L}_{\mathrm{j}} \mathrm{H}=\sum_{v=0}^{\mathrm{N}-1} z(t, x)^{v} \frac{\partial \mathrm{C}_{v}}{\partial t_{j}} \quad, \quad j=1, \ldots, \mathrm{m}
$$

Definimos a distribuição

$$
w=\mathrm{H}-\sum_{v=0}^{\mathrm{N}-1} z^{v} \mathrm{C}_{v}
$$

Observe que $\mathrm{L}_{0}^{\mathrm{N}}$ anula o somatório do segundo membro acima. De fato, pela regra da cadeia, e porque as distribuições $C_{v}$ continuam anulando $\partial_{x}$, temos para todo $k \in \mathbb{N}$ que

$$
\begin{aligned}
\mathrm{L}_{0}^{\mathrm{k}}\left(z^{v} \mathrm{C}_{v}\right)=\left(\mathrm{L}_{0}^{\mathrm{k}}\left(z^{v}\right)\right) \mathrm{C}_{v}=v\left(\mathrm{~L}_{0}^{\mathrm{k}-1}\left(z^{v-1} \mathrm{~L}_{0} z\right)\right) \mathrm{C}_{v}=v\left(\mathrm{~L}_{0}^{\mathrm{k}-1}\left(z^{v-1}\right)\right) \mathrm{C}_{v}= \\
=v(v-1)\left(\mathrm{L}_{0}^{\mathrm{k}-2}\left(z^{v-2} \mathrm{~L}_{0} z\right)\right) \mathrm{C}_{v}=v(v-1)\left(\mathrm{L}_{0}^{\mathrm{k}-2}\left(z^{v-2}\right)\right) \mathrm{C}_{v}=\cdots= \\
\quad=v(v-1) \ldots(v-\mathrm{k}+1)\left(\mathrm{L}_{0}^{v-\mathrm{k}}\left(z^{v-k}\right)\right) \mathrm{C}_{v}
\end{aligned}
$$

Se $k=N$, essa expressão vale 0 , porque $v$ varia de 0 a $N-1$. Então (2.49) acima permite concluir que $\mathrm{L}_{0}^{\mathrm{N}} \mathfrak{w}=\mathrm{L}_{0}^{\mathrm{N}} \mathrm{H}=u$. Por outro lado, para cada $j=1, \ldots, \mathrm{m}$ e cada $v=0, \ldots, N-1$, a regra da cadeia pode ser usada novamente para calcularmos

$$
\mathrm{L}_{\mathrm{j}}\left(z^{v} \mathrm{C}_{v}\right)=z^{v}\left(\mathrm{~L}_{\mathrm{j}} \mathrm{C}_{v}\right)=z^{v} \frac{\partial \mathrm{C}_{v}}{\partial \mathrm{t}_{j}}
$$

Segue que

$$
\mathrm{L}_{j} w=\mathrm{L}_{j} \mathrm{H}-\sum_{v=0}^{\mathrm{N}-1} z^{v} \frac{\partial \mathrm{C}_{v}}{\partial t_{j}}=\mathrm{L}_{j} \mathrm{H}-\mathrm{L}_{\mathrm{j}} \mathrm{H}=0
$$

Logo $\mathrm{L}_{0}^{\mathrm{N}} \mathfrak{w}=u$ e $\mathbb{L} w=0$, mas o que não sabemos agora é se $w$ é contínua. Para provar isso e finalizar o teorema, devido à equação (2.49), será suficiente provarmos que cada $C_{v}$ é contínua.

Aplique o teorema 2.41 à solução distribucional $w$ : diminuindo $\mathcal{B}_{\mathrm{r}^{\prime}} \times \mathrm{J}^{\prime}$ se necessário, podemos afirmar que existem $M \geq 0$ e $f_{1} \in C^{0}\left(\mathcal{B}_{r^{\prime}} \times J^{\prime}\right)$ tais que $w=\partial_{x}^{M} f_{1}$. Isso implica que existe $\mathrm{f}_{2} \in \mathrm{C}^{0}\left(\mathcal{B}_{\mathrm{r}^{\prime}} \times \mathrm{J}^{\prime}\right)$ tal que $\mathrm{H}-\mathrm{w}=\partial_{x}^{M} \mathrm{f}_{2}$ (empregando a notação que já utilizamos para primitivas de ordens superiores com relação a $x$, basta tomarmos $f_{2}=H^{-M}-f_{1}$, que é contínua porque $\mathrm{H}$ o é). Finalmente, a proposição 2.39 garante que existe $f_{3} \in \mathrm{C}^{0}\left(\mathcal{B}_{\mathrm{r}^{\prime}} \times \mathrm{J}^{\prime}\right)$ tal que $\mathrm{H}-w=\mathrm{L}_{0}^{M} \mathrm{f}_{3}$.

Por outro lado, sabemos que

$$
\mathrm{L}_{0}^{\mathrm{k}}(\mathrm{H}-\mathrm{w})=\sum_{v=\mathrm{k}}^{\mathrm{N}-1} \frac{v !}{(v-\mathrm{k}) !} z^{v-\mathrm{k}} \mathrm{C}_{v} \quad, \quad \mathrm{k}=0, \ldots, \mathrm{N}-1
$$


Dessa equação, podemos tirar cada $C_{N-k}$ em função de $C_{N-1}, C_{N-2}, \ldots, C_{N-(k-1)}$ :

$$
\begin{aligned}
& \mathrm{C}_{\mathrm{N}-1}=\frac{1}{(\mathrm{~N}-1) !} \mathrm{L}_{0}^{\mathrm{N}-1}(\mathrm{H}-\mathrm{w}) \\
& \mathrm{C}_{\mathrm{N}-\mathrm{k}}=\frac{1}{(\mathrm{~N}-\mathrm{k}) !}\left(\mathrm{L}_{0}^{\mathrm{N}-\mathrm{k}}(\mathrm{H}-\mathrm{w})-\sum_{v=1}^{\mathrm{k}-1} \frac{(\mathrm{N}-\mathrm{k}-\mathrm{v}) !}{v !} z^{v} \mathrm{C}_{\mathrm{N}-\mathrm{k}-\mathrm{v}}\right), \mathrm{k}=2, \ldots, \mathrm{N}-1
\end{aligned}
$$

Provemos que $\mathrm{C}_{\mathrm{N}-1}$ é contínua. Como

$$
\mathrm{C}_{\mathrm{N}-1}=\frac{1}{(\mathrm{~N}-1) !} \mathrm{L}_{0}^{\mathrm{N}-1}(\mathrm{H}-\mathrm{w})=\frac{1}{(\mathrm{~N}-1) !} \mathrm{L}_{0}^{\mathrm{M}+\mathrm{N}-1} \mathrm{f}_{3}
$$

a proposição 2.39 pode ser usada para concluirmos que

$$
\mathrm{C}_{\mathrm{N}-1}=\frac{\partial^{\mathrm{M}+\mathrm{N}-1}}{\partial x^{\mathrm{M}+\mathrm{N}-1}} \mathrm{f}_{4} \quad \text { em } \mathcal{B}_{\mathrm{r}^{\prime}} \times \mathrm{J}^{\prime}
$$

para alguma $f_{4} \in C^{0}\left(\mathcal{B}_{r^{\prime}} \times J^{\prime}\right)$. Mas $0=\partial_{x} C_{N-1}=\partial_{x}^{M+N_{f_{4}}}$, e um cálculo indutivo similar ao já feito anteriormente com o operador $\mathrm{L}_{0}$ mostra que, nesse caso,

$$
f_{4}=\sum_{\rho=0}^{M+N-1} x^{\rho} D_{\rho}
$$

para certas distribuições $D_{1}, \ldots, D_{M+N-1}$ que anulam $\partial_{x}$. Sendo $f_{4}$ contínua, podemos concluir que cada $D_{\rho}$ é uma função contínua de $t$. De fato, tome $M+N$ números distintos $\varepsilon_{1}, \ldots, \varepsilon_{M+N} \in J^{\prime}$. A equação (2.50) fornece

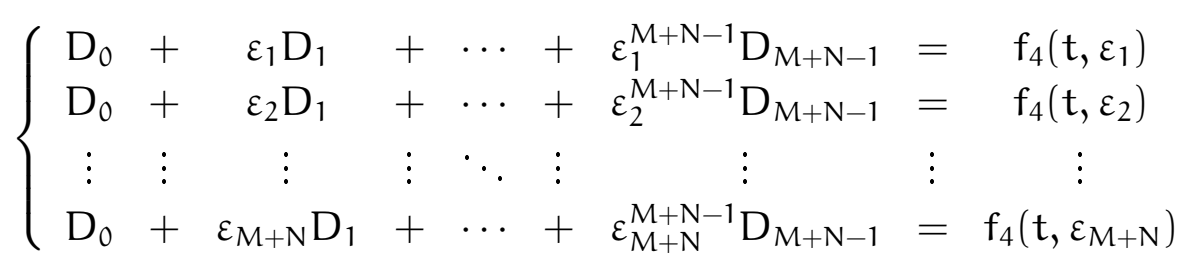

Esse sistema linear nas variáveis $\mathrm{D}_{\rho}$ é não-singular, devido à regra do Determinante de Vandermonde, e como $f_{4}\left(t, \varepsilon_{j}\right), j=1, \ldots, M+N$, são funções contínuas de $t$, segue da Regra de Cramer que cada $\mathrm{D}_{\rho}$ também o é. Em particular,

$$
C_{N-1}=\frac{\partial^{M+N-1} f_{4}}{\partial x^{M+N-1}}=(M+N-1) ! D_{M+N-1}
$$

também é uma função contínua de $t$, como havíamos afirmado.

Prosseguimos indutivamente para demonstrarmos a continuidade das outras $\mathrm{C}_{\mathrm{N}-\mathrm{k}}$ : suponha que já saibamos que $\mathrm{C}_{\mathrm{N}-1}, \ldots, \mathrm{C}_{\mathrm{N}-\mathrm{k}}$ são funções contínuas de t. Temos

$$
C_{N-(k+1)}=\frac{1}{(N-k-1) !}\left(L_{0}^{N-k-1} L_{0}^{M} f_{3}-\sum_{\nu=1}^{k} \frac{(N-k-1-v) !}{v !} z^{v} C_{N-k-1-v}\right)
$$


O termo $L_{0}^{N-k-1} L_{0}^{M} f_{3}$ pode ser escrito na forma $\partial_{x}^{M+N-k-1} f_{5}$ para alguma função contínua $f_{5}$, e o somatório acima é uma função contínua por hipótese de indução. Tomando uma primitiva desse somatório de ordem $M+N-k-1$ com relação a $x$, podemos dizer que

$$
C_{N-(k+1)}=\frac{\partial^{M+N-k-1}}{\partial x^{M+N-k-1}} f_{6} \quad \text { em } \mathcal{B}_{r^{\prime}} \times J^{\prime}
$$

para alguma $f_{6} \in \mathrm{C}^{0}\left(\mathcal{B}_{\mathrm{r}^{\prime}} \times \mathrm{J}^{\prime}\right)$, e agora procedemos exatamente como fizemos para $\mathrm{C}_{\mathrm{N}-1}$ para concluir que $C_{N-(k+1)}$ é uma função contínua de $t$.

\subsection{Consequências para hipoelipticidade}

Os teoremas 2.13 e 2.47 nos permitirão agora caracterizar a hipoelipticidade analítica local da estrutura $\mathbb{L}$.

Teorema 2.51. Sejam $\mathbb{L}$ uma estrutura involutiva analitica sobre $\Omega$ e $\omega_{0} \in \Omega$. Denote por $\mathrm{F}$ a fibra de $\mathbb{L}$ passando por $\omega_{0}$, em algum aberto. São equivalentes:

a) $\mathbb{L}$ não é analítica hipoelíptica em $\omega_{0}$.

b) Existe uma vizinhança aberta $\mathrm{U} \ni \omega_{0}$ onde está definida uma integral primeira $z$ tal que $z(\mathrm{U})$ não é uma vizinhança aberta de $z\left(\omega_{0}\right)$.

c) Existe uma vizinhança aberta $\mathrm{V} \ni \omega_{0}$ onde está definida uma função $\mathrm{u} \in \mathrm{C}^{\infty}(\mathrm{V})$ flat em $\mathrm{V} \cap \mathrm{F}$ tal que $\mathbb{L} u=0$ e $\mathrm{u} \in \mathrm{C}^{\omega}(\mathrm{V} \backslash \mathrm{F})$.

d) Dado $k \in \mathbb{N}$, existe uma vizinhança aberta $V \ni \omega_{0}$ onde está definida uma função $\mathrm{u} \in \mathrm{C}^{\mathrm{k}}(\mathrm{V})$ tal que $\mathbb{L} \mathrm{u}=0$ e $\mathrm{u} \notin \mathrm{C}^{\mathrm{k}+1}\left(\mathrm{~V}^{\prime}\right)$ para qualquer $\omega_{0} \in \mathrm{V}^{\prime} \subseteq \mathrm{V}$.

Em particular, $\mathbb{L}$ é analítica hipoelíptica em $\omega_{0}$ se e somente se $\mathbb{L}$ é aberta em $\omega_{0}$.

Demonstração: As implicações $d \Rightarrow a$ e $c \Rightarrow a$ são imediatas. Provaremos que $a \Rightarrow b$, $\mathrm{b} \Rightarrow \mathrm{c}$ e $\mathrm{b} \Rightarrow \mathrm{d}$, e isto já será suficiente para concluirmos todas as equivalências:

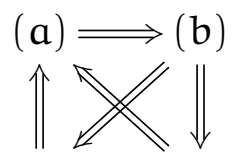

(d)

(c)

Iniciamos com $a \Rightarrow b$. Podemos assumir que $\Omega \subseteq \mathbb{R}^{m+1}$, com $\omega_{0}=0$, e que $\mathbb{L}$ é gerada em $\Omega$ por campos analíticos $L_{1}, \ldots, L_{m}$. Suponha que $b$ é falsa, e sejam $U \ni 0$ e $u \in D^{\prime}(U)$ tais que $f_{j}=L_{j} u$ é analítica em $U$ para cada $j$. Queremos provar que $u$ é analítica em alguma vizinhança aberta $V \ni 0$. O teorema 1.62 pode ser aplicado para obtermos uma solução 
analítica $v$ do sistema $L_{j} v=f_{j}, j=1, \ldots, m$, em algum aberto $W$ em torno da origem (as condições de compatibilidade seguem do fato que os campos $L_{j}$ comutam). Mas então $u-v$ é solução de $\mathbb{L}$ em $W$, e sabemos do teorema 2.47 que, após diminuirmos $W$, podemos encontrar um operador $L_{0}$ a coeficientes analíticos tal que $u-v=L_{0}^{N_{f}}$ para algum $N \in \mathbb{N}$ e alguma $f$ solução contínua de $\mathbb{L}$. O teorema 2.13 também afirma que $f=\tilde{f} \circ z$, para alguma integral primeira $z$ e alguma $\tilde{f}$ contínua em $z(\bar{W})$ e holomorfa em $z(\bar{W})^{\circ}$. Defina $\mathrm{V}=z^{-1}\left(z(\bar{W})^{\circ}\right)$. Considerando-se o corolário 2.18, como estamos assumindo que $\mathrm{b}$ é falsa, temos que $V$ é uma vizinhança aberta da origem. $E$ em $V$ temos $f=\tilde{f} \circ z$ analítica, logo $u-v=\mathrm{L}_{0}^{N} f$ também, e finalmente $u=v+(u-v)$ também, como queríamos.

Agora demonstremos ambas $\mathrm{b} \Rightarrow \mathrm{c}$ e $\mathrm{b} \Rightarrow \mathrm{d}$. Aqui podemos assumir que as coordenadas em $\Omega$ em torno de $\omega_{0}$ são tais que $L_{j}$ e $z$ são dados como em (2.3) e (2.4), com $\omega_{0}=0$. Tomemos também $\mathcal{B}_{\mathrm{r}}$ e $\mathrm{J}$ como antes. Devido à proposição 2.9, sabemos que $z\left(\mathcal{B}_{\mathrm{r}} \times \mathrm{J}\right)$ omite pelo menos um dos dois semieixos abertos $\{\mathfrak{R} \zeta=0, \mathfrak{I} \zeta>0\}$ e $\{\mathfrak{R} \zeta=0, \mathfrak{I} \zeta<0\}$ em $\mathbb{C}$. Em particular, para qualquer $\alpha \in \mathbb{R}$, podemos definir um ramo de $z(t, x)^{\alpha}$ contínuo em $\mathcal{B}_{\mathrm{r}} \times \mathrm{J}$ e analítico em $\left(\mathcal{B}_{\mathrm{r}} \times \mathrm{J}\right) \backslash \mathrm{F}$, onde $\mathrm{F}=z^{-1}(0)$ é a fibra de $\mathbb{L}$ passando por 0 .

Para provar c, suponha sem perda de generalidade que o semieixo imaginário omitido por $z$ é $\{\mathfrak{R} \zeta=0, \mathfrak{I} \zeta<0\}$, e defina

$$
u(t, x)=e^{-z(t, x)^{-1 / 4}}
$$

Fora de $\mathrm{F}, \mathrm{u}$ é a composição de uma função holomorfa $\operatorname{com} z$, logo é uma solução de $\mathbb{L}$ em $\left(\mathcal{B}_{\mathrm{r}} \times \mathrm{J}\right) \backslash \mathrm{F}$. Como $\mathrm{F}$ tem medida nula, u é uma solução distribucional de $\mathbb{L}$ em $\mathcal{B}_{\mathrm{r}} \times \mathrm{J}$. Agora, se $D$ é uma derivação de qualquer ordem com respeito às variáveis $(t, x)$, então

$$
\operatorname{Du}(t, x)=\sum_{j} a_{j}\left(P_{j} z(t, x)\right) z(t, x)^{-\rho_{j}} e^{-z(t, x)^{-1 / 4}}
$$

onde $\Sigma_{j}$ é uma soma finita, para certas constantes $a_{j} \in \mathbb{C}$ e $\rho_{j}>0$ e certos operadores diferenciais parciais $P_{j}$. Para vermos que $u$ é flat em $F$, devemos provar que $\lim _{(t, x) \rightarrow\left(t_{0}, x_{0}\right)} \operatorname{Du}(t, x)=0$, para todo $\left(t_{0}, x_{0}\right) \in F$. O termo $P_{j} z(t, x)$ tende a $P_{j} z\left(t_{0}, x_{0}\right)$ e não atrapalha a convergência. Essencialmente precisamos provar que

$$
\lim _{\zeta \rightarrow 0} \zeta^{-\rho} e^{-\zeta^{-1 / 4}}=0
$$

para um dado $\rho>0$ e para $\zeta$ na imagem de $z$. Considere a forma polar $\zeta=\operatorname{Re}^{i \theta}$. Tendo-se em vista que $z$ omite o semieixo imaginário negativo, tem-se $-\pi / 2<\theta<3 \pi / 2$. Logo,

$$
\left|\zeta^{-\rho} e^{-\zeta^{-1 / 4}}\right|=\mathrm{R}^{-\rho} e^{-\left(\mathrm{R}^{-1 / 4}\right) \cos (\theta / 4)}
$$

Esse número tende a 0 quando $R \rightarrow 0$ porque $\cos (\theta / 4)$ é positivo para $-\pi / 2<\theta<3 \pi / 2$.

Para provar d, defina

$$
u(t, x)=z(t, x)^{k+\frac{1}{2}}
$$


Como acima, u é solução distribucional de $\mathbb{L}$ em $\mathcal{B}_{\mathrm{r}} \times \mathrm{J}$. Por outro lado, para qualquer vizinhança $\mathcal{B}_{\mathrm{r}}^{\prime} \times \mathrm{J}^{\prime} \subseteq \mathcal{B} \times \mathrm{J}$ da origem, as derivadas complexas de $\zeta^{\mathrm{k}+1 / 2}$ estão definidas até a ordem $k$, mas não depois disso, no conjunto $z\left(\mathcal{B}_{r}^{\prime} \times J^{\prime}\right)$. De fato, a derivada de ordem $k+1$ não é contínua na origem. Concluímos então que $u \in C^{k}\left(\mathcal{B}_{r} \times J\right) \operatorname{mas} u \notin C^{k+1}\left(\mathcal{B}_{r}^{\prime} \times J^{\prime}\right)$.

\subsection{Hipoelipticidade parcial das soluções}

Seja $\mathbb{L}$ uma estrutura gerada por campos analíticos $L_{1}, \ldots, L_{m}$ sobre um aberto da forma $\mathcal{B} \times \mathrm{J}, \mathcal{B} \subseteq \mathbb{R}^{\mathrm{m}}, \mathrm{J} \subseteq \mathbb{R}$, e suponha que os $\mathrm{L}_{\mathrm{j}}$ são dados em sua forma canônica local, isto é,

$$
\mathrm{L}_{j}=\frac{\partial}{\partial t_{j}}+\lambda_{j}(t, x) \frac{\partial}{\partial x}
$$

Uma propriedade das soluções de $\mathbb{L}$, que é utilizada pelos autores em [BT] para provar o teorema 2.47, é a hipoelipticidade parcial com relação às variáveis $t$, que significa o seguinte: se $u \in \mathcal{D}^{\prime}(\mathcal{B} \times J)$ é solução de $\mathbb{L}$ e se $\mathcal{B}^{\prime} \subset \subset \mathcal{B}$ e $\mathrm{J}^{\prime} \subset \subset \mathrm{J}$, então $u$ induz uma aplicação

$$
\mathrm{F}: \mathcal{B}^{\prime} \rightarrow \mathcal{D}^{\prime}\left(\mathrm{J}^{\prime}\right)
$$

Ainda mais, F é suave, como aplicação a valores num espaço vetorial topológico, o que por definição significa que todas suas derivadas são contínuas. (Por derivada $\partial_{t_{j}}$ de uma aplicação $\mathrm{G}: \mathrm{U} \rightarrow \mathrm{E}$, onde $\mathrm{U} \subseteq \mathbb{R}^{\mathrm{m}}$ é aberto e $\mathrm{E}$ é um espaço vetorial topológico, entende-se a aplicação $\partial_{t_{j}} G: U \rightarrow E$ definida por

$$
\partial_{t_{j}} G(t)=\lim _{h \rightarrow 0} \frac{G\left(t_{1}, \ldots, t_{j}+h, \ldots, t_{m}\right)-G\left(t_{1}, \ldots, t_{j}, \ldots, t_{m}\right)}{h}
$$

quando esse limite existir. As derivadas $\partial^{\alpha} G, \alpha \in \mathbb{N}^{m}$, são definidas recursivamente de maneira natural.)

Apesar de não termos utilizado esta propriedade em nossa demonstração, ela está presente em vários trabalhos da área de equações lineares, e tem importância suficiente para ser provada agora.

Seja $u \in \mathcal{D}^{\prime}(\mathcal{B} \times J)$ qualquer. Para cada $\psi \in \mathrm{C}_{\mathrm{c}}^{\infty}(\mathrm{J})$ está definida uma distribuição $\mathrm{U}_{\psi} \in \mathcal{D}^{\prime}(\mathcal{B})$ dada por

$$
\left\langle\mathrm{u}_{\psi}, \phi\right\rangle=\langle\mathrm{u}, \phi \otimes \psi\rangle \quad, \quad \phi \in \mathrm{C}_{\mathrm{c}}^{\infty}(\mathcal{B})
$$

Se $\mathbb{L} u=0$ em $\mathcal{B} \times J$, fixe abertos arbitrários $\mathcal{B}^{\prime} \subset \subset \mathcal{B}$ e J' $\subset \subset$ J. Vimos na prova do teorema 2.41 que é possível definir, para cada $\psi \in \mathrm{C}_{\mathrm{c}}^{\infty}\left(\mathrm{J}^{\prime}\right)$, uma função contínua $\mathrm{f}_{\psi}: \mathcal{B}^{\prime} \rightarrow \mathbb{C}$ dada por

$$
f_{\psi}(t)=\frac{1}{(2 \pi)^{m}} \int_{\mathbb{R}^{\prime}} \int_{\mathbb{R}^{m}} \hat{u}(\xi, \eta) e^{i \xi \xi} \breve{\psi}(\eta) d \xi d \eta
$$


para uma certa $U \in \mathcal{D}^{\prime}\left(\mathbb{R}^{m+1}\right)$. Vimos também a seguinte propriedade desta função:

$$
\langle u, \phi \otimes \psi\rangle=\int f_{\psi}(t) \phi(t) d t \quad, \quad \phi \in C_{c}^{\infty}\left(\mathcal{B}^{\prime}\right)
$$

Isto é, $\mathrm{f}_{\psi}=\mathrm{U}_{\psi}$ como distribuições. Note também que, como a equação

$$
\left\langle u, \phi \otimes\left(\psi_{1}+\lambda \psi_{2}\right)\right\rangle=\left\langle u, \phi \otimes \psi_{1}\right\rangle+\lambda\left\langle u, \phi \otimes \psi_{2}\right\rangle
$$

vale para quaisquer $\psi_{1}, \psi_{2} \in \mathrm{C}_{\mathrm{c}}^{\infty}\left(\mathrm{J}^{\prime}\right), \lambda \in \mathbb{C}$ e $\phi \in \mathrm{C}_{\mathrm{c}}^{\infty}\left(\mathcal{B}^{\prime}\right)$, temos

$$
f_{\psi_{1}+\lambda \psi_{2}}=f_{\psi_{1}}+\lambda f_{\psi_{2}} \text { quase sempre em } \mathcal{B}^{\prime}
$$

Proposição 2.55. Nas condições acima, se $\mathrm{u} \in \mathrm{H}_{\mathrm{loc}}^{\mathrm{r}, \mathrm{s}}\left(\mathcal{B}^{\prime} \times \mathrm{J}^{\prime}\right)$, então $\mathrm{U}_{\psi} \in \mathrm{H}_{\mathrm{loc}}^{\mathrm{r}}\left(\mathcal{B}^{\prime}\right)$ para toda $\psi \in \mathrm{C}_{\mathrm{c}}^{\infty}\left(\mathrm{J}^{\prime}\right)$.

Demonstração: Seja $\mathcal{B}^{\prime \prime} \subset \subset \mathcal{B}^{\prime}$ arbitrário, e seja $J^{\prime \prime} \subset \subset J^{\prime}$ contendo supp $\psi$. Pelo corolário 2.32, existe $w \in H^{r, s}$ que coincide com $u$ em $\mathcal{B}^{\prime \prime} \times J^{\prime \prime}$. Seja $W_{\psi} \in \mathcal{D}^{\prime}\left(\mathbb{R}^{m}\right)$ definida por

$$
\left\langle W_{\psi}, \phi\right\rangle=\langle w, \phi \otimes \psi\rangle \quad, \quad \phi \in C_{c}^{\infty}\left(\mathbb{R}^{m}\right)
$$

Observe que $\mathrm{U}_{\psi}=\mathrm{W}_{\psi}$ em $\mathcal{B}^{\prime \prime}$, pois $u=w$ em $\mathcal{B}^{\prime \prime} \times \mathrm{J}^{\prime \prime} \supseteq \mathcal{B}^{\prime \prime} \times \operatorname{supp} \psi$. Resta apenas vermos que $W_{\psi} \in \mathrm{H}^{r}$.

Escolha $\sigma \in \mathbb{R}$ tal que $\left(1+\eta^{2}\right)^{2 \sigma-s}$ seja integrável na reta. Como $\check{\psi} \in \mathcal{S}$, existe $C \geq 0$ tal que

$$
|\psi(\breve{\eta})| \leq C\left(1+\eta^{2}\right)^{\sigma} \quad, \quad \eta \in \mathbb{R}
$$

Para qualquer $\phi \in \mathcal{S}\left(\mathbb{R}^{\mathrm{m}}\right)$, temos

$$
\begin{aligned}
\left\langle\widehat{W}_{\psi}, \phi\right\rangle=\left\langle W_{\psi}, \hat{\phi}\right\rangle=\langle w, \hat{\phi} \otimes \psi\rangle & = \\
& =\left\langle w,(\phi \otimes \breve{\psi})^{\wedge}\right\rangle=\langle\hat{w}, \phi \otimes \breve{\psi}\rangle=\iint \hat{w}(\xi, \eta) \phi(\xi) \check{\psi}(\eta) d \xi d \eta
\end{aligned}
$$

Isto é, a transformada de $W_{\psi}$ é a função mensurável

$$
\widehat{W}_{\psi}(\xi)=\int \widehat{w}(\xi, \eta) \check{\psi}(\eta) d \eta \quad, \quad \xi \in \mathbb{R}^{m}
$$


Agora a Desigualdade de Minkowski integral e a Desigualdade de Hölder implicam que

$$
\begin{aligned}
& \left\|W_{\psi}\right\|_{H^{r}}=\left(\int\left(1+|\xi|^{2}\right)^{r}\left|\int \hat{w}(\xi, \eta) \check{\psi}(\eta) d \eta\right|^{2} d \xi\right)^{1 / 2} \\
& \leq \int\left(\int|\hat{w}(\xi, \eta)|^{2}|\breve{\psi}(\eta)|^{2}\left(1+|\xi|^{2}\right)^{r} d \xi\right)^{1 / 2} d \eta \\
& \leq C \int\left(\int|\hat{w}(\xi, \eta)|^{2}\left(1+\eta^{2}\right)^{2 \sigma}\left(1+|\xi|^{2}\right)^{r} d \xi\right)^{1 / 2} d \eta \\
& =C \int\left(1+\eta^{2}\right)^{\sigma-\frac{s}{2}}\left(\int|\hat{w}(\xi, \eta)|^{2}\left(1+\eta^{2}\right)^{s}\left(1+|\xi|^{2}\right)^{r} d \xi\right)^{1 / 2} d \eta \\
& \leq \mathrm{C}\left(\int\left(1+\eta^{2}\right)^{2 \sigma-s} \mathrm{~d} \eta\right)^{1 / 2}\left(\iint|\hat{w}(\xi, \eta)|^{2}\left(1+\eta^{2}\right)^{s}\left(1+|\xi|^{2}\right)^{r} \mathrm{~d} \xi \mathrm{d} \eta\right)^{1 / 2} \\
& =C\left(\int\left(1+\eta^{2}\right)^{2 \sigma-s} d \eta\right)^{1 / 2}\|w\|_{H^{r, s}}<\infty
\end{aligned}
$$

Corolário 2.56. Se $u \in \mathcal{D}^{\prime}(\mathcal{B} \times J)$ e $\mathbb{L} u=0$, então $\mathrm{f}_{\psi} \in \mathrm{C}^{\infty}\left(\mathcal{B}^{\prime}\right)$ para toda $\psi \in \mathrm{C}_{\mathrm{c}}^{\infty}(\mathrm{J})$.

Demonstração: Sejam $\mathcal{B}^{\prime \prime} \subset \subset \mathcal{B}^{\prime}$ e $J^{\prime \prime} \subset \subset J^{\prime}$ arbitrários. Sabemos (proposição 2.40) que $u \in \mathrm{H}_{\mathrm{loc}}^{\mathrm{r}, \mathrm{s}}\left(\mathcal{B}^{\prime} \times \mathrm{J}^{\prime}\right)$ para $\mathrm{r}$ arbitrariamente grande e $s$ negativo o suficiente. Então a proposição anterior e o fato que $\cap_{p \in \mathbb{R}} H_{\text {loc }}^{p}\left(\mathcal{B}^{\prime \prime}\right)=C^{\infty}\left(\mathcal{B}^{\prime \prime}\right)$ implicam que $f_{\psi}=U_{\psi} \in C^{\infty}\left(\mathcal{B}^{\prime \prime}\right)$, isto é, singsupp $f_{\psi} \subseteq \mathcal{B}^{\prime} \backslash \mathcal{B}^{\prime \prime}$. Mas como $\mathcal{B}^{\prime}$ era arbitrário, podemos concluir que singsupp $f_{\psi}=\emptyset$, como queríamos.

Finalmente podemos definir a aplicação $\mathrm{F}$ como em (2.52). Se $u \in \mathcal{D}^{\prime}(\mathcal{B} \times \mathrm{J})$ é solução de $\mathbb{L}$, fixe mais uma vez $\mathcal{B}^{\prime} \subset \subset \mathcal{B}$ e $J^{\prime} \subset \subset$ J e seja

$$
\mathrm{F}: \mathcal{B}^{\prime} \rightarrow \mathcal{D}^{\prime}\left(\mathrm{J}^{\prime}\right) \quad, \quad\langle\mathrm{F}(\mathrm{t}), \psi\rangle=\mathrm{f}_{\psi}(\mathrm{t}) \quad, \quad \psi \in \mathrm{C}_{\mathrm{c}}^{\infty}\left(\mathrm{J}^{\prime}\right)
$$

Para cada $t \in \mathcal{B}^{\prime}$ fixo, a linearidade de $F(t)$ segue de $(2.54)$, e se uma sequência $\left(\psi_{j}\right)$ converge em $C_{c}^{\infty}\left(J^{\prime}\right)$ a 0 , então $\left(\breve{\psi}_{j}\right)$ converge em $\mathcal{S}\left(J^{\prime}\right)$ a 0 , e em particular também suas normas $\mathrm{H}^{-s}$, onde s é tal que $u \in \mathrm{H}_{\text {loc }}^{\mathrm{r}, \mathrm{s}}\left(\mathcal{B}^{\prime} \times \mathrm{J}^{\prime}\right)$; logo a continuidade de $\mathrm{F}(\mathrm{t})$ (como funcional linear de $\left.C_{c}^{\infty}\left(J^{\prime}\right)\right)$ é consequência da estimativa (2.42). Por fim, se $\alpha \in \mathbb{N}^{m}$, segue da equação (2.53) que a derivada $\partial^{\alpha} \mathrm{F}$ é dada pela aplicação contínua

$$
\mathrm{t} \mapsto\left(\partial^{\alpha} \mathrm{F}\right)(\mathrm{t}) \quad: \quad \psi \mapsto \partial^{\alpha} \mathrm{f}_{\psi}(\mathrm{t})
$$

Portanto, F é uma aplicação bem-definida e suave, como havíamos afirmado. 


\section{CAPÍTULO 3 \\ Regularidade Analítica Global}

Neste capítulo abordaremos o artigo [BZ], que trata do problema análogo ao de [BT], mas agora no caso global. A partir daqui, $\Omega$ representará uma variedade analítica $p a$ racompacta orientável de dimensão $n+1$, sobre a qual consideraremos uma estrutura involutiva analítica $\mathbb{L}$ de posto $n$. As hipóteses destacadas são pouco restritivas, mas precisam ser assumidas para que possamos utilizar um resultado da teoria homológica de feixes de funções analíticas, uma ferramenta imprescindível quando tivermos de estender funções analíticas em $\Omega$.

\subsection{Conjuntos de nível}

Seja $p \in \Omega$ qualquer. Sabemos, pelos resultados vistos em [BT], que existe uma carta local $\left(U,\left(t_{1}, \ldots, t_{n}, x\right)\right)$ centrada em $p$ onde $\mathbb{L}$ é gerada pelos campos

$$
\mathrm{L}_{j}=\frac{\partial}{\partial \mathrm{t}_{j}}+\lambda_{j}(\mathrm{t}, \mathrm{x}) \frac{\partial}{\partial x} \quad, \quad j=1, \ldots, n
$$

sendo cada $\lambda_{j}$ uma função analítica em $U$, e existe uma integral primeira analítica em $U$ dada na forma

$$
z_{p}(t, x)=x+i \phi(t, x)
$$

onde $\phi$ é analítica e satisfaz $\phi(0, \cdot) \equiv 0 \mathrm{em} \mathrm{U}$.

O princípio da constância local demonstrado naquele artigo afirma que, se $u$ é contínua e $\mathbb{L} u=0$ em $U$, então existem um aberto $V_{p} \ni p$ e $\tilde{u}: z_{p}\left(V_{p}\right) \rightarrow \mathbb{C}$ contínua em $z_{p}\left(V_{p}\right)$ e holomorfa em $z_{p}\left(V_{p}^{\circ}\right)$ tais que $u=\tilde{u} \circ z_{p}$ em $\bigvee_{p}$. Em geral, não sabemos se 
$0=z_{p}(0)$ pertence à fronteira de $z_{\mathfrak{p}}\left(V_{p}\right)$, e portanto pode não fazer sentido perguntar-se se ũ é holomorfa em torno de 0 . Mas nossa primeira observação é que esse será o caso sob uma hipótese a mais:

Proposição 3.1. Nas condições acima, se u é analítica em torno de p, então ũ é holomorfa em torno de 0.

Demonstração: Para cada $(0, x) \in V$, temos $u(0, x)=\tilde{u}\left(z_{p}(0, x)\right)=\tilde{u}(x)$. Então a função real

$$
\tilde{u}_{0}=\left.\tilde{u}\right|_{z_{p}\left(v_{p}\right) \cap\{\Im \zeta=0\}}
$$

é analítica perto de 0. Estenda-a a uma função, de mesmo nome, holomorfa em um aberto $W \subseteq \mathbb{C}$ contendo a origem, e seja

$$
v=\tilde{u}_{0} \circ z_{p}
$$

Como $z_{p}$ é uma integral primeira e $\tilde{u}_{0}$ é holomorfa, temos $\mathbb{L} v=0$ pelo teorema 1.8. Note que também $v(0, x)=\tilde{u}_{0}(x)=\tilde{u}(x)=u(0, x)$. Então, pelo corolário 1.61, $u=v$ em algum aberto $W^{\prime} \subseteq W$ contendo a origem. Isso implica que $\tilde{u} \circ z_{p}=\tilde{u}_{0} \circ z_{p}$ em $W^{\prime}$, isto é, $\tilde{u}=\tilde{u}_{0}$, e ũ é holomorfa em torno de 0 .

Definição 3.2. Considere a seguinte relação de equivalência em $\Omega$ : dois pontos $\mathrm{p}, \mathrm{q}$ estão relacionados se e somente se existem pontos $\mathrm{p}=\mathrm{p}_{1}, \mathrm{p}_{2}, \ldots, \mathrm{p}_{\mathrm{r}}=\mathrm{q}$ tais $q u e$

- $z_{\mathfrak{p}_{j}}^{-1}(0)$ é conexo por caminhos, $j=1, \ldots, r$;

- $z_{\mathfrak{p}_{j}}^{-1}(0) \cap z_{\mathfrak{p}_{j+1}}^{-1}(0) \neq \emptyset, j=1, \ldots, r-1$.

onde $z_{p_{j}}$ é uma integral primeira em torno de $p_{j}$ tal como acima. As classes de equivalência dessa relação se chamam conjuntos de nivel de $\mathbb{L}$.

Segue que conjuntos de nível são conexos por caminhos, e eles coincidem com as componentes conexas de uma integral primeira global, caso esta exista.

Exemplo 3.3. Seja $\mathbb{T}^{2}=\mathbb{S}_{\mathrm{t}}^{1} \times \mathbb{S}_{x}^{1}$ o toro plano, isto é, $\mathbb{R}^{2}=\{(\mathrm{t}, x)\}$ quocientado pela relação de equivalência

$$
\left(t_{1}, x_{1}\right) \sim\left(t_{2}, x_{2}\right) \Longleftrightarrow t_{1}-t_{2}, x_{1}-x_{2} \in\{2 k \pi ; k \in \mathbb{Z}\}
$$

Denote por $\pi: \mathbb{R}^{2} \rightarrow \mathbb{T}^{2}$ a aplicação quociente. Sejam $\mathbb{L}_{1}$ e $\mathbb{L}_{2}$ estruturas em $\mathbb{T}^{2}$ geradas respectivamente pelos push-forward a $\mathbb{T}^{2}$ dos campos $\mathrm{L}_{1}$ e $\mathrm{L}_{2}$ dados por

$$
\mathrm{L}_{1}=\frac{\partial}{\partial \mathrm{t}} \quad, \quad \mathrm{L}_{2}=\frac{\partial}{\partial \mathrm{t}}+\alpha \frac{\partial}{\partial x}
$$

onde $\alpha \in \mathbb{R}$ é irracional. Os conjuntos de nivel de $\mathbb{L}_{1}$ são os paralelos do toro, isto é, a imagem por $\pi$ das retas $\left\{\left(t, x_{0}\right) \in \mathbb{R}^{2} ; t \in \mathbb{R}\right\}$, para cada $x_{0}$ fixado. De fato, 
numa carta local com as coordenadas $(t, x)$ canônicas do toro, uma integral primeira local para $\mathbb{L}_{1}$ é dada por $z_{1}(\mathrm{t}, \mathrm{x})=x$ (apenas local, por não ser periódica), e suas fibras são os conjuntos $\{x=$ constante $\}$. Consideradas como retas em $\mathbb{R}^{2}$, são todas paralelas ao eixo t. Isso prova que dois pontos quaisquer em um mesmo paralelo do toro sempre pertencem a uma fibra de uma única integral primeira como $z_{1}$, enquanto que dois pontos em paralelos distintos nunca poderão estar relacionados com respeito à relação que define os conjuntos de nivel de $\mathbb{L}_{1}$. Note que aqui os conjuntos de nivel são fechados e localmente conexos.

Da mesma forma, os conjuntos de nivel de $\mathbb{L}_{2}$ são a imagem por $\pi$ das retas $\left\{(t, x) \in \mathbb{R}^{2} ; x=\alpha t+\alpha_{0}\right\}$, para cada $\alpha_{0} \in \mathbb{R}$, porque, numa carta local com as coordenadas $(\mathrm{t}, \mathrm{x})$ canônicas do toro, uma integral primeira local para $\mathbb{L}_{2}$ é dada por $z_{2}(t, x)=x-\alpha t$. Em constraste com $\mathbb{L}_{1}$, aqui os conjuntos de nivel não são fechados nem localmente conexos: eles são densos em $\mathbb{T}^{2}$. Para simplificar a notação, provaremos esta afirmação apenas para $\pi\left(\left\{(t, x) \in \mathbb{R}^{2} ; x=\alpha t\right\}\right)$. O que temos de provar é que, dados $\varepsilon>0$ e $\left(t_{0}, x_{0}\right) \in \mathbb{R}^{2}$, existem $k, l \in \mathbb{Z}$ e $t \in \mathbb{R}$ tais que

$$
\left|(t, \alpha t)-\left(t_{0}+2 k \pi, x_{0}+2 l \pi\right)\right|<\varepsilon
$$

Seja $\sigma: \mathbb{R} \rightarrow \mathbb{S}^{1}$ dada por $\sigma(y)=\exp (2 \pi i y)$. Seja arg $: \mathbb{C} \rightarrow[0,2 \pi)$ a função argumento. Considere a família de retas $r_{m}=\left\{\left(t_{0}+2 m \pi, x\right) \in \mathbb{R}^{2} ; x \in \mathbb{R}\right\}, m \in \mathbb{Z}$. A reta $x=\alpha$ cruza $r_{m}$ no ponto $p_{m}=\left(t_{m}, x_{m}\right)=\left(t_{0}+2 m \pi, \alpha t_{0}+2 \alpha m \pi\right)$. Note que, se $\mathrm{m}_{1} \neq \mathrm{m}_{2}$, então $\arg \sigma\left(x_{\mathrm{m}_{1}}\right) \neq \arg \sigma\left(x_{\mathrm{m}_{2}}\right)$. De fato, se o contrário fosse verdade então $2 \alpha \pi\left(m_{2}-m_{1}\right)=x_{m_{2}}-x_{m_{1}}$ seria múltiplo de $2 \pi$, contradizendo nossa escolha de $\alpha$.

Fixe $\mathrm{n} \in \mathbb{N}$ tal que $(2 \pi) / \mathrm{n}<\varepsilon$. Pelo princípio das casas dos pombos, sendo que os subconjuntos $\sigma[0,(2 \pi) / n], \ldots, \sigma[(n-1)(2 \pi) / n, 2 \pi)$ de $\mathbb{S}^{1}$ representam as casas, e os infinitos pontos $\sigma\left(x_{m}\right)$ representam os pombos, existem $m_{1}<m_{2} \in \mathbb{Z}$ tais que

$$
0<\left|\arg \sigma\left(x_{m_{2}}\right)-\arg \sigma\left(x_{m_{1}}\right)\right| \leq \frac{2 \pi}{n}
$$

Defina $\mathrm{d}=\arg \sigma\left(x_{m_{2}}\right)-\arg \sigma\left(x_{m_{1}}\right)$ e $m_{j}=m_{2}+(j-2)\left(m_{2}-m_{1}\right), j=3,4, \ldots$, e observe que

$$
\arg \sigma\left(x_{m_{j+1}}\right)-\arg \sigma\left(x_{m_{j}}\right)=\mathrm{d} \text { ou } 2 \pi+d \quad \text { para todo } j
$$

Isto é consequência do fato que $x_{m_{j+1}}-x_{m_{j}}=x_{m_{2}}-x_{m_{1}}$ para todo $j$, que é verdadeiro porque $\left(\mathrm{m}_{1}, \mathrm{~m}_{2}, \ldots\right)$ é uma progressão aritmética.

Assim, se $M \in \mathbb{N}$ é maior que $\mathrm{d}$, os pontos $\arg \sigma\left(x_{\mathrm{m}_{1}}\right), \ldots, \arg \sigma\left(\mathrm{x}_{\mathrm{m}_{\mathrm{M}}}\right)$ dividem $[0,2 \pi)$ em vários subintervalos de comprimento menor ou igual a d. Em particular existe j tal que

$$
\left|\arg \sigma\left(x_{0}\right)-\arg \sigma\left(x_{m_{j}}\right)\right|<d
$$

Isto é, $x_{0}$ e $x_{m_{j}}$ se escrevem na forma

$$
x_{0}=2 n_{0} \pi+r_{1} \quad, \quad x_{m_{j}}=2 n_{j} \pi+r_{j}
$$


para certos $n_{0}, n_{j} \in \mathbb{Z}$ e $r_{1}, r_{j} \in[0,2 \pi)$ satisfazendo $\left|r_{0}-r_{j}\right|<d$. Então tome $t=t_{m_{j}}$, $\mathrm{k}=\mathrm{m}_{\mathrm{j}}$ e $\mathrm{l}=\mathrm{n}_{\mathrm{j}}-\mathrm{n}_{\mathrm{o}}$ em (3.4). Temos assim

$$
\begin{aligned}
& \left|(t, \alpha t)-\left(t_{0}+2 k \pi, x_{0}+2 l \pi\right)\right|=\left|\left(t_{m_{j}}, x_{m_{j}}\right)-\left(t_{0}+2 m_{j} \pi, x_{0}+2\left(n_{j}-n_{0}\right) \pi\right)\right|= \\
& \quad=\left|\left(t_{m_{j}}, 2 n_{j} \pi+r_{j}\right)-\left(t_{m_{j}}, 2 n_{0} \pi+r_{0}+2\left(n_{j}-n_{0}\right) \pi\right)\right|=\left|r_{j}-r_{0}\right|<|d| \leq(2 \pi) / n<\varepsilon
\end{aligned}
$$

como queríamos.

Exemplo 3.5. No cilindro $\mathbb{R}_{\mathrm{t}} \times \mathbb{S}_{x}^{1}$, os conjuntos de nivel da estrutura gerada pelo campo

$$
\mathrm{L}=\mathrm{t} \frac{\partial}{\partial \mathrm{t}}+\frac{\partial}{\partial x}
$$

são localmente conexos, mas em geral não fechados. De fato, se fosse um campo em $\mathbb{R}^{2}$, estaria definida uma integral primeira global

$$
z(t, x)=t e^{-x}
$$

$e$ os conjuntos de nivel seriam gráficos de exponenciais, isto é, $\left\{t=t_{0} e^{x}\right\}$, com $t_{0} \in \mathbb{R}$. No cilindro, esta integral primeira é apenas local, e então os conjuntos de nivel serão os mesmos gráficos de exponenciais, mas agora enrolados ao redor do cilindro. Dessa forma, o círculo $\mathrm{F}_{0}=\{(0, x) ; x \in[0,2 \pi]\}$ é o único conjunto de nivel compacto, enquanto que todos os outros tendem a $\mathrm{F}_{0}$ (quando $\mathrm{x} \rightarrow-\infty$ ), tanto pelo lado $\mathrm{t}>0$ como pelo lado $\mathrm{t}<0$.

Lema 3.6. Sejam $p \in \Omega$ um ponto e $W \subseteq \Omega$ uma vizinhança aberta de $p$. Se $u \in \mathcal{D}^{\prime}(\mathrm{W})$ é solução de $\mathbb{L} u=0$, então existem um aberto $\mathrm{U} \subseteq \mathrm{W}$ contendo $\mathrm{p}$ e uma solução $w$ continua em $\mathrm{U}$ (isto é, $\mathbb{L} w=0$ em $\mathrm{U}$ ) de forma que $\mathrm{u}=\mathrm{P} w$ para algum operador diferencial parcial linear $\mathrm{P}$ com coeficientes analiticos. Ainda mais, w é analítica em qualquer aberto de $\mathrm{U}$ onde u seja analítica.

Este lema, de autoria de Trèves, pode ser visto como um refinamento do teorema 2.47, a única diferença sendo a propriedade de $w$ ser analítica sempre que $u$ o for. $O$ operador P obtido no teorema 2.47 não era elíptico, portanto essa propriedade pode não valer ali. A demonstração do lema acima, omitida no presente trabalho, se encontra em [T2], teorema II.5.1, p. 100, onde o autor obtém P elíptico. Aplicaremos este resultado à demonstração da propriedade mais importante dos conjuntos de nível de $\mathbb{L}$ : eles propagam analiticidade. Mais precisamente:

Teorema 3.7. Sejam $\mathrm{F}$ um conjunto de nivel de $\mathbb{L}$ e $\mathrm{u} \in \mathcal{D}^{\prime}(\Omega)$ uma distribuição tal que $\mathrm{Lu} \in \mathrm{C}^{\omega}(\Omega)$ para toda seção $\mathrm{L}$ de $\mathbb{L}$. Se u é analítica em algum ponto de $\mathrm{F}$, então $u$ é analitica em qualquer ponto de $\mathrm{F}$.

Demonstração: Seja $p \in F$ arbitrário e sejam $z_{p}$ e $V_{p}$ como definidos acima. Defina

$$
\mathrm{F}_{\mathrm{p}}=z_{\mathrm{p}}^{-1}(0) \subseteq \mathrm{F}
$$


Diminua $V_{p}$ se necessário para encontrar $u_{p} \in C^{\omega}\left(V_{p}\right)$ satisfazendo $\mathbb{L}\left(u-u_{p}\right)=0$ em $V_{p}$. (Isto é possível porque, por hipótese, Lu é analítica para qualquer seção $L$ de $\mathbb{L}$ e em particular para os campos $L_{j}$ que geram $\mathbb{L}$ em $V_{p}$; tudo o que precisamos fazer é encontrar uma solução analítica $u_{p}$ do sistema $L_{j} u_{p}=L_{j} u$.) Pelo lema acima, após diminuir $V_{p}$ novamente, encontramos $w_{p}$ contínua em $V_{p}$ tal que $\mathbb{L} w_{p}=0$ e $u-u_{p}=P w_{p}$ para algum operador a coeficientes analíticos, de forma que, dado qualquer aberto $W \subseteq V_{p}$, temos $u \in C^{\omega}(W)$ se e somente se $w_{p} \in C^{\omega}(W)$. Pelo principio da constância local, após diminuir $V_{p}$ uma terceira vez, podemos assumir que existe $\tilde{w}_{p}$ contínua em $z_{p}\left(V_{p}\right)$ e holomorfa em $z_{p}\left(V_{p}\right)^{\circ}$ tal que

$$
w_{p}=\tilde{w}_{p} \circ z_{p} \quad \text { em } V_{p}
$$

Mais ainda, a proposição 3.1 garante que $\tilde{w}_{p}$ é holomorfa na origem se $w_{p}$ for analítica em p. Em resumo, o que deduzimos até agora é que para cada $p \in F$ existem uma vizinhança aberta $V_{p}$ e:

- uma integral primeira $z_{p} \in \mathrm{C}^{\omega}\left(\mathrm{V}_{\mathrm{p}}\right)$;

- uma função $w_{p} \in C^{0}\left(V_{p}\right)$ que é analítica em $V_{p}$ precisamente onde $u$ é analítica;

- uma função $\tilde{w}_{p} \in C^{0}\left(z_{p}\left(V_{p}\right)\right)$ que satisfaz $w_{p}=\tilde{w}_{p} \circ z_{p}$, e que é holomorfa na origem se $w_{p}$ for analítica em $p$.

Sejam agora $a, b \in F$ quaisquer, e suponha que $u$ como no enunciado é analítica em $a$. Queremos provar que $u$ é analítica em $b$. Pela definição dos conjuntos de nível, existe um caminho contínuo contido em F ligando a a b. Por compacidade deste caminho, existem finitos pontos $a=p_{1}, p_{2}, \ldots, p_{r}=b$ cujas vizinhanças correspondentes $V_{p_{j}}$ o recobrem. Se reordenarmos os índices $j$ e eliminarmos aquelas $V_{p_{j}}$ que estão contidas em alguma $V_{p_{k}}$, também podemos assumir que $V_{p_{j}} \cap V_{p_{j+1}} \neq \emptyset, j=1, \ldots, r-1$. Como feito acima, defina $\mathrm{F}_{\mathrm{p}_{j}}=z_{\mathrm{p}_{j}}^{-1}(0)=\mathrm{V}_{\mathrm{p}_{j}} \cap \mathrm{F}$.

Tome $x \in F_{p_{1}} \cap F_{p_{2}}$. Como $u$ é analítica em $p_{1}=a, w_{p_{1}}$ também o é, logo sabemos que $\tilde{w}_{p_{1}}$ é holomorfa em torno da origem. Como $z_{p_{1}}(x)=0$ (porque $x \in F_{p_{1}}$ ), a equação $w_{p_{1}}=\tilde{w}_{p_{1}} \circ z_{p_{1}}$ implica então que $w_{p_{1}}$, logo também $u$, são analíticas em $\chi$. Mas $\chi$ também pertence a $V_{p_{2}}$, e assim $w_{p_{2}}$ é analítica em $x$. Agora apliquemos o princípio da constância local a $w_{p_{2}}$ em $x$, mas com relação à integral primeira $z_{p_{2}}$ : em algum aberto $\mathrm{Z} \subseteq \mathrm{V}_{\mathrm{p}_{2}}$, podemos escrever

$$
w_{\mathrm{p}_{2}}=v \circ z_{\mathrm{p}_{2}}
$$

para alguma $v$ contínua em $z_{\mathrm{p}_{2}}(W)$. Por um lado, a proposição 3.1 nos diz que $v$ é holomorfa em torno da origem, já que $w_{p_{2}}$ é analítica em $x$. Por outro, a decomposição

$$
w_{p_{2}}=\tilde{w}_{p_{2}} \circ z_{p_{2}}
$$


vale em $V_{p_{2}} \supseteq W$. Concluímos que $\tilde{w}_{p_{2}}=v$ em $z_{p_{2}}(W) \ni 0$, e assim $\tilde{w}_{p_{2}}$ é holomorfa na origem. Por fim, dado que $z_{p_{2}}\left(p_{2}\right)=0$, a equação (3.8) acima permite concluir que $w_{p_{2}}$, e portanto $u$, são analíticas em $p_{2}$. A partir daqui, prosseguimos por indução para concluir que $u$ é analítica em $p_{3}, p_{4}, \ldots, p_{r}=b$.

\subsection{Hipoelipticidade analítica global}

O conceito que estamos interessados em caracterizar é o seguinte:

Definição 3.9. A estrutura $\mathbb{L}$ é dita globalmente analítica hipoelíptica (GAH) se, para toda distribuição $u \in \mathcal{D}^{\prime}(\Omega)$, a seguinte implicação é verdadeira:

$$
\mathrm{Lu} \in \mathrm{C}^{\omega}(\Omega) \text { para toda seção } \mathrm{L} \text { de } \mathbb{L} \Rightarrow \mathrm{u} \in \mathrm{C}^{\omega}(\Omega)
$$

De acordo com o teorema 2.51, a propriedade local análoga a esta, num dado $p \in \Omega$, é equivalente à integral primeira $z_{p}$ ser uma aplicação aberta em $p$, e isto torna o próximo resultado quase trivial:

Teorema 3.10. Se qualquer conjunto de nivel contém pelo menos um ponto onde $\mathbb{L}$ é aberta, então $\mathbb{L}$ é GAH.

Demonstração: Seja $u \in \mathcal{D}^{\prime}(\Omega)$ tal que $L u \in C^{\omega}(\Omega)$ para toda seção $L$ de $\mathbb{L}$. Sejam $p \in \Omega$ e $F$ o conjunto de nível de $\mathbb{L}$ que contém $p$. Por hipótese, existe um ponto $q \in F$ onde $\mathbb{L}$ é aberta, e isto implica que u é analítica em q, pelo teorema 2.51. Então basta aplicarmos o teorema 3.7 para concluir que $u$ é analítica em $p$.

O que veremos a partir de agora é que, sob certas hipóteses topológicas a respeito dos conjuntos de nível de $\mathbb{L}$, a recíproca deste teorema é verdadeira. Mas antes é necessário introduzir noções cohomológicas a respeito de feixes de funções analíticas.

Seja $\mathcal{U}=\left\{U_{i}\right\}_{i \in I}$ uma cobertura de $\Omega$ por abertos $U_{i}$, indexada em algum conjunto de índices I. Definimos

$$
\mathcal{C}^{1}\left(\mathcal{U}, C^{\omega}\right)=\left\{\left(g_{i j}\right)_{u_{i} \cap u_{j} \neq \emptyset} ; g_{i j} \in C^{\omega}\left(U_{i} \cap U_{j}\right)\right\}
$$

Isto é, $\mathcal{C}^{1}\left(\mathcal{U}, C^{\omega}\right)$ é o conjunto de todas as famílias $\left(g_{i j}\right)$ de funções analíticas em $U_{i} \cap U_{j}$, considerando-se apenas os pares $i, j$ tais que $U_{i} \cap U_{j} \neq \emptyset$. Seja também

$$
\mathcal{Z}^{1}\left(\mathcal{U}, C^{\omega}\right)=\left\{\left(g_{i j}\right) \in \mathcal{C}^{1}\left(\mathcal{U}, C^{\omega}\right) ; g_{i j}+g_{j k}=g_{i k} \text { em } U_{i} \cap \mathcal{U}_{j} \cap \mathcal{U}_{k}\right\}
$$

Este é o espaço dos cociclos relativos à cobertura $\mathcal{U}$. Observemos que um cociclo necessariamente satisfaz $g_{i i}=0$ e $g_{i j}=-g_{j i}$. Defina ainda

$$
\mathcal{C}^{0}\left(\mathcal{U}, \mathrm{C}^{\omega}\right)=\left\{\left(g_{i}\right)_{i \in \mathrm{I}} ; g_{i} \in C^{\omega}\left(U_{i}\right)\right\}
$$


Existe uma aplicação natural $\delta: \mathcal{C}^{0}\left(\mathcal{U}, C^{\omega}\right) \rightarrow \mathcal{C}^{1}\left(\mathcal{U}, C^{\omega}\right)$, definida por

$$
\delta\left(\left(g_{i}\right)_{i \in I}\right)=\left(g_{i}-g_{j}\right) u_{i} \cap u_{j} \neq \emptyset
$$

Ou seja, definimos a imagem de uma família $\left(g_{i}\right)$ como sendo a família $\left(g_{i}-g_{j}\right)$ de funções analíticas em $\mathrm{U}_{i} \cap \mathrm{U}_{j}$, com $i, j$ variando em $\mathrm{I}$ e tais que $\mathrm{U}_{i} \cap \mathrm{U}_{j} \neq \emptyset$. Note que esta família é um cociclo. Finalmente, defina também

$$
\mathcal{B}^{1}\left(\mathcal{U}, C^{\omega}\right)=\delta\left(\mathcal{C}^{0}\left(\mathcal{U}, C^{\omega}\right)\right)
$$

Em particular, $\mathcal{B}^{1}\left(\mathcal{U}, C^{\omega}\right) \subseteq \mathcal{Z}^{1}\left(\mathcal{U}, C^{\omega}\right)$. Os conjuntos $\mathcal{C}^{0}, \mathcal{C}^{1}, \mathcal{Z}^{1}, \mathcal{B}^{1}$ são espaços vetoriais sobre $\mathbb{C}$, com a soma e produto por escalar usuais de funções analíticas feitos coordenada a coordenada. A aplicação $\delta$ é linear, e o espaço quociente

$$
\mathcal{H}^{1}\left(\mathcal{U}, C^{\omega}\right)=\frac{\mathcal{Z}^{1}\left(\mathcal{U}, C^{\omega}\right)}{\mathcal{B}^{1}\left(\mathcal{U}, C^{\omega}\right)}
$$

é o chamado primeiro grupo de cohomologia de $\mathcal{U}$ com coeficientes complexos.

Teorema 3.11. Se $\Omega$ é uma variedade analítica paracompacta orientável, então $\mathcal{H}^{1}\left(\mathcal{U}, \mathrm{C}^{\omega}\right)=0$ para qualquer cobertura $\mathcal{U}$ de $\Omega$ por abertos.

Isto é, qualquer cociclo $\left(g_{i j}\right)$ pode ser escrito como imagem por $\delta$ de uma família de funções analíticas $\left(h_{i}\right)$ :

$$
g_{i j}=h_{i}-h_{j} \quad \text { em } u_{i} \cap u_{j}
$$

A demonstração está presente em [C], no contexto de variedades de Stein, enquanto que o teorema $3 \mathrm{em}[\mathrm{G}]$ prova que é suficiente considerarmos variedades paracompactas orientáveis.

Definição 3.12. O suporte singular analítico de uma distribuição $v \in \mathcal{D}^{\prime}(\Omega)$, denotado por $\operatorname{singsupp}_{A} v$, é o complementar do maior aberto onde $v$ é uma função analítica.

Lema 3.13. Se um conjunto de nivel $\mathrm{F}$ é localmente conexo, então para cada $\mathrm{p} \in \mathrm{F}$ existe uma vizinhança aberta $V_{p} \ni p$ onde está definida uma integral primeira $z_{p}$ de forma que

$$
\mathrm{F} \cap \mathrm{V}_{\mathrm{p}}=z_{\mathrm{p}}^{-1}(0)
$$

Demonstração: Sejam $p \in F$ um ponto e $V_{p} \ni p$ uma vizinhança aberta onde está definida uma integral primeira $z_{p}$ tal que $z_{p}(p)=0$. Por hipótese, podemos tomar $V_{p}$ de forma que $\mathrm{F} \cap \mathrm{V}_{\mathrm{p}}$ seja conexo. Isso implica que é também conexo por caminhos, devido à primeira condição imposta na definição dos conjuntos de nível de $\mathbb{L}$. Seja $F_{p}=z_{p}^{-1}(0) \subseteq F$, e suponha por absurdo que $\left(F \backslash F_{p}\right) \cap V_{p} \neq \emptyset$. 
O conjunto de nível $F$ é por definição uma reunião arbitrária dos conjuntos de nível nulos de integrais primeiras locais. Como estamos assumindo que $\left(F \backslash F_{p}\right) \cap V_{p} \neq \emptyset$, existe uma integral primeira $w$ em algum aberto $V \subseteq V_{p}$ tal que $w^{-1}(0) \subseteq F \backslash F_{p}$. Pela proposição 2.15, $z_{p}$ e $w$ são constantes nas fibras uma da outra em $V$ (após diminuirmos $V$ se necessário); $\left.\log w\right|_{V} ^{-1}(0)=\left.z_{\mathfrak{p}}\right|_{V} ^{-1}\left(\zeta_{0}\right)$, para algum $\zeta_{0} \in \mathbb{C}, \zeta_{0} \neq 0$.

Podemos conectar $p$ a um ponto qualquer $\left.q \in w\right|_{V} ^{-1}(0)$ por um caminho $\gamma$ contido em F. Como acima, $\gamma$ é formado por uma reunião arbitrária de conjuntos de nível nulos de integrais primeiras locais, e, utilizando-se novamente a proposição 2.15 para compararmos $z_{\mathfrak{p}}$ com cada uma destas, concluímos que $z_{\mathfrak{p}}$ é localmente constante em $\gamma$. Sendo $\gamma$ conexo, deduz-se que $z_{p}$ é constante em $\gamma$, e em particular $\zeta_{0}=0$, absurdo.

Proposição 3.14. Seja $\mathrm{F}$ um conjunto de nivel localmente conexo de $\mathbb{L}$, e suponha que $\mathbb{L}$ não é aberta em nenhum ponto de $\mathrm{F}$. Então existem um aberto $\mathrm{W} \supseteq \mathrm{F}$ e uma distribuição $w \in \mathcal{D}^{\prime}(\mathrm{W})$ tais que $\operatorname{singsupp}_{\mathrm{A}} w=\mathrm{F}$ e $\mathrm{L} w \in \mathrm{C}^{\omega}(\mathrm{W})$ para toda seção $\mathrm{L}$ de $\mathbb{L}$.

O fato da estrutura não ser aberta em $p$ implica que existe em torno de $p$ uma solução singular. Então o que esta proposição diz é que, se temos uma solução singular local em torno de cada ponto de um conjunto de nível localmente conexo, podemos "colar" todas elas e obter uma distribuição $w$ cuja singularidade está em F e cuja imagem pela estrutura $\mathbb{L}$ é analítica. Essas propriedades são quase o que precisamos para mostrar que $\mathbb{L}$ não é GAH; o que está faltando é obter $w$ definida não apenas em torno de $F$, mas sim em $\Omega$.

Demonstração de 3.14. Existem $V_{p}$ e $z_{p}, p$ percorrendo $F$, tais como dadas no lema 3.13, e por hipótese $z_{\mathfrak{p}}$ não é aberta em $p$ para nenhum $p \in F$. Dessa forma, tal como feito no teorema 2.51, sabemos que a imagem $z_{\mathfrak{p}}\left(\mathrm{V}_{\mathrm{p}}\right)$ omite pelo menos um dos semieixos imaginários $\{\mathfrak{R} \zeta=0, \mathfrak{I} \zeta>0\}$ e $\{\mathfrak{R} \zeta=0, \Im \zeta<0\}$, e podemos definir a função analítica

$$
u_{p}=\log z_{p} \quad \text { em } V_{p} \backslash F
$$

Também podemos considerar $u_{p}$ como uma função $L_{\text {loc }}^{1}$ em $V_{p}$, uma vez que só não está definida em $F \cap V_{p}=z_{p}^{-1}(0)$, um conjunto de medida nula. Como log é uma função holomorfa onde está definida, temos $\mathbb{L} u_{p}=0$. Agora defina $u_{p, q}=u_{p}-u_{q}$ em cada interseção $V_{p} \cap V_{q}$ não-vazia. $O$ lema 3.13 garante que, em $F \cap\left(V_{p} \cap V_{q}\right)$, temos $z_{p}=0$ ou $z_{\mathrm{q}}=0$, e que, fora de $F$, ambas $z_{\mathrm{p}}$ e $z_{\mathrm{q}}$ são não-nulas. Assim ambas $u_{\mathrm{p}}=\log z_{\mathrm{p}}$ e $u_{\mathrm{q}}=\log z_{\mathrm{q}}$ são analíticas, e $u_{p, q} \in C^{\omega}\left(\left(V_{p} \cap V_{q}\right) \backslash F\right)$. Mostremos que na verdade $u_{p, q} \in C^{\omega}\left(V_{p} \cap V_{q}\right)$.

Seja então $s \in F \cap\left(V_{p} \cap V_{q}\right)$. Pelo teorema 2.15, existem abertos $V \subseteq V_{p} \cap V_{q}$ em torno de $\mathrm{s}, \mathrm{A} \subseteq \mathbb{C}$ em torno de $z_{\mathrm{p}}(\mathrm{s})=0$ e $\mathrm{B} \subseteq \mathbb{C}$ em torno de $z_{\mathrm{q}}(\mathrm{s})=0$ e existe um biholomorfismo $h: A \rightarrow B$ tais que $z_{p}=h \circ z_{q}$ em V. Note que $h(0)=h\left(z_{q}(s)\right)=z_{p}(s)=0$. Diminua $A$ se necessário de forma que

$$
h(\zeta)=\zeta g(\zeta) \quad \text { em } A
$$


para alguma função holomorfa g que não se anula em $A$. Isto pode ser obtido porque 0 é um zero simples de $h$, já que esta é um biholomorfismo. Note que, em particular, log g é uma função holomorfa. Dessa forma, para todo $r \in V$,

$$
\begin{aligned}
& u_{\mathrm{p}, \mathrm{q}}(\mathrm{r})=\log \left(z_{\mathrm{p}}(\mathrm{r})\right)-\log \left(z_{\mathrm{q}}(\mathrm{r})\right)=\log \left(\mathrm{h}\left(z_{\mathrm{q}}(\mathrm{r})\right)\right)-\log \left(z_{\mathrm{q}}(\mathrm{r})\right)= \\
& =\log \left(z_{\mathrm{q}}(\mathrm{r}) \mathrm{g}\left(z_{\mathrm{q}}(\mathrm{r})\right)\right)-\log \left(z_{\mathrm{q}}(\mathrm{r})\right)=\log \left(\mathrm{g}\left(z_{\mathrm{q}}(\mathrm{r})\right)\right)
\end{aligned}
$$

isto é, $u_{p, q}$ é analítica em $V$, e assim também em $s$, como queríamos.

Ponha $W=\cup_{p \in F} V_{p}$. Está claro que a família das funções analíticas $u_{p, q}$, com $p, q$ variando em $F$, define um cociclo relativo à cobertura $\left\{V_{p}\right\}_{p \in F}$ de $W$. Então o teorema 3.11 pode ser aplicado para concluirmos que existem $w_{p} \in C^{\omega}\left(V_{p}\right)$ satisfazendo

$$
u_{p}-u_{q}=w_{q}-w_{p} \quad \text { em } v_{p} \cap v_{q} \quad, \quad p, q \in F
$$

Dessa forma, $w: W \rightarrow \mathbb{C}$ dada por

$$
w=u_{p}+w_{p} \quad \text { em } v_{p}
$$

está bem definida, é $L_{\text {loc }}^{1}$ em $W$ e analítica em $W \backslash F$, isto é, $\operatorname{singsupp}_{A} w=F$. Veja também que $L w \in C^{\omega}(W)$ para qualquer seção $L$ de $\mathbb{L}$, já que $u_{p}$ satisfaz essa mesma propriedade e $w_{p}$ é analítica.

Corolário 3.15. Se F na proposição acima for também fechado, então w pode ser obtida em $\mathcal{D}^{\prime}(\Omega)$.

Demonstração: Com a notação da proposição anterior, considere a cobertura de $\Omega$ por abertos $\mathcal{U}=\{\mathrm{W}, \Omega \backslash \mathrm{F}\}$. Como $\mathcal{w} \in \mathrm{C}^{\omega}(\mathrm{W} \backslash \mathrm{F})$, a família $\{w\}$ define um cociclo de $\mathcal{U}$. Assim, pelo teorema 3.11, existem $u_{1} \in C^{\omega}(W)$ e $u_{2} \in C^{\omega}(\Omega \backslash F)$ tais que

$$
u_{1}-u_{2}=w \quad \text { em } W \backslash F
$$

Então podemos definir $v: \Omega \rightarrow \mathbb{C}$ por

$$
v=\left\{\begin{array}{ccc}
w-u_{1} & \text { em } & W \\
-u_{2} & \text { em } & \Omega \backslash F
\end{array}\right.
$$

Teremos $v \in \mathrm{L}_{\text {loc }}^{1}(\Omega)$, $\operatorname{singsupp}_{A} v=\mathrm{F}$ e $\mathrm{L} v \in \mathrm{C}^{\omega}(\Omega)$ para cada seção $\mathrm{L}$ de $\mathbb{L}$, como queríamos.

Chegamos finalmente à caracterização da hipoelipticidade analítica global em termos dos conjuntos de nível de $\mathbb{L}$ :

Teorema 3.16. (Bergamasco-Zani) Se cada conjunto de nivel de $\mathbb{L}$ é fechado e localmente conexo, então $\mathbb{L}$ é GAH se e somente se cada conjunto de nível contém pelo menos um ponto onde $\mathbb{L}$ é aberta. 
Demonstração: Resta mostrarmos que GAH implica que cada conjunto de nível possui um ponto onde $\mathbb{L}$ é aberta. Suponha, pela contrapositiva, que existe um conjunto de nível $F$ tal que $\mathbb{L}$ não é aberta em nenhum ponto $p \in F$. Diretamente do corolário 3.15, obtemos $w \in \mathrm{L}_{\text {loc }}^{1}(\Omega)$ tal que $w \notin \mathrm{C}^{\omega}(\Omega)$ mas $\mathrm{L} w \in \mathrm{C}^{\omega}(\Omega)$ para qualquer seção $\mathrm{L}$ de $\mathbb{L}$, o que é precisamente a negação da propriedade GAH.

Observação 3.17. Vimos anteriormente exemplos de estruturas para as quais a hipótese dos conjuntos de nível serem fechados e localmente conexos não são válidas, $e$ nesse caso a caracterização provada acima pode não ser verdadeira. Considere o toro $\mathbb{T}^{2}$, com o campo $\mathrm{L}_{2}$ dado como no exemplo 3.3, isto é,

$$
\mathrm{L}_{2}=\frac{\partial}{\partial t}+\alpha \frac{\partial}{\partial x}
$$

$\operatorname{com} \alpha \in \mathbb{R} \backslash \mathbb{Q}$. Sabe-se que nesse caso a hipoelipticidade analítica global da estrutura em questão está relacionada ao fato de a ser um número de Liouville exponencial, e não mais apenas ao fato de que cada conjunto de nivel contém um ponto onde a estrutura é aberta. Veja [Gr]. 


\section{Teorema de Aproximação de Baouendi-Trèves}

Estruturas localmente integráveis admitem, por definição, uma família local $Z_{1}, \ldots, Z_{m}$ de integrais primeiras em torno de cada ponto. Levando-se em conta a linearidade da estrutura e a regra da cadeia, do fato das $Z_{j}$ serem soluções da estrutura conclui-se que qualquer polinômio nas $Z_{j}$ a coeficientes constantes também o é. O que demonstraremos agora é uma ferramenta útil que permite aproximar localmente por polinômios deste tipo qualquer solução suave da estrutura. O mesmo resultado vale também com soluções distribucionais, mas sua demonstração envolve detalhes da teoria de distribuições que fogem ao escopo deste projeto. A demonstração desenvolvida aqui segue as mesmas linhas do capítulo 2 de $[\mathrm{BCH}]$. As letras $\mathrm{m}, \mathrm{n}$ e $\mathrm{N}$ denotarão inteiros positivos tais que $\mathrm{N}=\mathrm{m}+\mathrm{n}$.

Estabelecemos também uma notação conveniente: se $\zeta=\left(\zeta_{1}, \ldots, \zeta_{\mathrm{m}}\right) \in \mathbb{C}^{\mathrm{m}}$, escrevemos $[\zeta]^{2}$ para denotar o número complexo

$$
\zeta_{1}^{2}+\cdots+\zeta_{m}^{2}
$$

Lema 4.1. A função é é Lipschitz com constante 1 no semiplano complexo $\mathfrak{R} \zeta \leq 0$.

Demonstração: Fixados $\zeta_{1}, \zeta_{2} \in \mathbb{C}$ com parte real não-positiva, defina $f:[0,1] \rightarrow \mathbb{C}$ por $f(s)=e^{\zeta_{2}+s\left(\zeta_{1}-\zeta_{2}\right)}$. A Desigualdade do Valor Médio implica que existe $s_{0} \in[0,1]$ tal que

$$
\left|e^{\zeta_{2}}-e^{\zeta_{1}}\right|=|\mathbf{f}(0)-\mathbf{f}(1)| \leq\left|\mathbf{f}^{\prime}\left(s_{0}\right)\right|=\left|\zeta_{1}-\zeta_{2}\right|\left|e^{\zeta_{2}+s_{0}\left(\zeta_{1}-\zeta_{2}\right)}\right| \leq\left|\zeta_{1}-\zeta_{2}\right|
$$

uma vez que $\mathfrak{R}\left(\zeta_{2}+s_{0}\left(\zeta_{1}-\zeta_{2}\right)\right)=s_{0} \mathfrak{R} \zeta_{1}+\left(1-s_{0}\right) \mathfrak{R} \zeta_{2} \leq 0$ 
A prova dos dois próximos lemas segue as ideias de [H], seção 3.4, p. 84.

Lema 4.2. Seja $M \in M_{m}(\mathbb{R})$ uma matriz real positiva definida e simétrica. Então

$$
\int_{\mathbb{R}^{\mathrm{m}}} e^{-(M x) \cdot x} \mathrm{~d} x=\frac{\pi^{\mathrm{m} / 2}}{\sqrt{\operatorname{det} M}}
$$

onde o produto que aparece na exponencial é o produto interno usual do espaço euclidiano.

Demonstração: O valor da integral

$$
\int_{-\infty}^{\infty} e^{-t^{2}} d t=\sqrt{\pi}
$$

é bem conhecido. Pode ser deduzido considerando-se sua análoga em dimensões maiores

$$
\mathrm{I}_{\mathrm{N}}=\int_{\mathbb{R}^{N}} e^{-|t|^{2}} \mathrm{dt}
$$

que pelo Teorema de Fubini é igual a $\mathrm{I}_{1}^{\mathrm{N}}$. A integral $\mathrm{I}_{2}$ é facilmente calculada por coordenadas polares, e permite deduzir o valor das demais $\mathrm{I}_{\mathrm{N}}$.

Através de uma mudança de variáveis, temos

$$
\int_{-\infty}^{\infty} e^{-a t^{2}} d t=\int_{-\infty}^{\infty} e^{-t^{2}} \frac{d t}{\sqrt{a}}=\sqrt{\frac{\pi}{a}}
$$

para todo $a>0$. Logo, se $D=\operatorname{diag}\left(a_{1}, \ldots, a_{m}\right)$ é uma matriz diagonal com termos positivos,

$$
\begin{aligned}
\int_{\mathbb{R}^{\mathrm{m}}} e^{-(\mathrm{Dx}) \cdot x} \mathrm{~d} x=\int_{\mathbb{R}^{\mathrm{m}}} e^{-\mathrm{a}_{1} x_{1}^{2}-\cdots-a_{\mathrm{m}} x_{\mathrm{m}}^{2}} \mathrm{~d} x=\int_{\mathbb{R}^{\mathrm{m}}} e^{-\mathrm{a}_{1} x_{1}^{2}} \cdots e^{-\mathrm{a}_{\mathrm{m}} x_{\mathrm{m}}^{2}} \mathrm{~d} x= \\
=\int_{-\infty}^{\infty} e^{-\mathrm{a}_{1} x_{1}^{2}} \mathrm{~d} x_{1} \cdots \int_{-\infty}^{\infty} e^{-\mathrm{a}_{\mathrm{m}} x_{\mathrm{m}}^{2}} \mathrm{~d} x_{\mathrm{m}}=\sqrt{\frac{\pi}{\mathrm{a}_{1}}} \cdots \sqrt{\frac{\pi}{a_{\mathrm{m}}}}=\frac{\pi^{\mathrm{m} / 2}}{\sqrt{\operatorname{det} \mathrm{D}}}
\end{aligned}
$$

Agora, se $M$ é uma matriz como no enunciado do lema, é sabido que podemos diagonalizála através de uma matriz de mudança de base ortogonal. Isto é, existem $A, D \in M_{m}(\mathbb{R})$ tais que $M=A^{-1} D A$, com $A$ ortogonal e $D$ diagonal. Em particular, $\operatorname{det} A= \pm 1$ e $\operatorname{det} M=\operatorname{det} D$. Mais ainda, sendo $M$ definida positiva, $D$ também o será, o que implica que os elementos de sua diagonal são todos positivos. Sendo assim, o resultado já foi provado para $\mathrm{D}$, e podemos usá-lo para concluir a tese para $M$ através de uma mudança de variáveis na integral:

$$
\begin{aligned}
\int e^{-(M x) \cdot x} d x=\int e^{-\left(A^{-1} D A x\right) \cdot x} d x=\int e^{-(D A x) \cdot(A x)} d x= \\
=\int e^{-(D y) \cdot y}\left|\operatorname{det} A^{-1}\right| d y=\int e^{-(D y) \cdot y} d y=\frac{\pi^{m / 2}}{\sqrt{\operatorname{det} D}}=\frac{\pi^{m / 2}}{\sqrt{\operatorname{det} M}}
\end{aligned}
$$


Lema 4.3. Seja $M \in M_{m}(\mathbb{C})$ uma matriz complexa simétrica com parte real positiva definida. Então

$$
\int_{\mathbb{R}^{m}} e^{-\langle M x, x\rangle} d x=\frac{\pi^{m / 2}}{\sqrt{\operatorname{det} M}}
$$

onde o produto que aparece na exponencial é o produto interno usual do espaço $\mathbb{C}^{m}$, e o ramo da raiz quadrada complexa utilizado é aquele que é positivo no semieixo real positivo.

Demonstração: Seja $\mathcal{H}_{m}$ o conjunto das matrizes $M_{m}(\mathbb{C})$ simétricas com parte real positiva definida.

Primeira afirmação: A função det não se anula em $\mathcal{H}_{\mathrm{m}}$.

De fato, sejam $A \in \mathcal{H}_{m}$ e $x \in \mathbb{C}^{m}$ arbitrários tais que $A x=0$. Vamos provar que $x=0$. Temos:

$$
\begin{aligned}
0=\mathfrak{R}\langle A x, x\rangle & =\mathfrak{R}\left\langle\left(\sum_{j=1}^{m} a_{1 j} x_{j}, \cdots, \sum_{j=1}^{m} a_{m j} x_{j}\right), x\right\rangle \\
& =\Re \sum_{j, k=1}^{m} a_{k j} x_{j} \overline{x_{k}} \stackrel{(*)}{=} \mathfrak{R}\left(\sum_{j<k} a_{k j}\left(x_{j} \overline{x_{k}}+x_{k} \overline{x_{j}}\right)+\sum_{l=1}^{m} a_{l l}\left(x_{l} \overline{x_{l}}\right)\right) \\
& =\sum_{j<k}\left(\Re a_{k j}\right)\left(x_{j} \overline{x_{k}}+x_{k} \overline{x_{j}}\right)+\sum_{l=1}^{m}\left(\Re a_{l l}\right)\left(x_{l} \overline{x_{l}}\right) \\
& =\sum_{j, k=1}^{m}\left(\Re a_{k j}\right)\left(x_{j} \overline{x_{k}}\right)=\langle(\Re A) x, x\rangle
\end{aligned}
$$

Então $x=0$, porque $\mathfrak{R A}$ é positiva definida. Note que a hipótese de $A$ ser simétrica foi essencial em $(*)$.

Segunda afirmação: $\mathcal{H}_{\mathrm{m}}$ é um conjunto convexo.

De fato, se $A, B \in \mathcal{H}_{m}$ e $s \in[0,1]$, devemos provar que $A+s(B-A) \in \mathcal{H}_{m}$. Esta matriz é simétrica porque $A, B$ o são, e só resta provar que tem parte real positiva definida. Mas, se $x \in C^{m}$, então

$$
\begin{aligned}
\langle[\mathfrak{R}(\mathrm{A}+\mathrm{s}(\mathrm{B}-\mathrm{A}))] x, x\rangle=\langle(\mathfrak{R A}) x, x\rangle+s(\langle(\mathfrak{R B}) x, x\rangle-\langle(\mathfrak{R A}) x, x\rangle)= \\
=s\langle(\mathfrak{R B}) x, x\rangle+(1-s)\langle(\mathfrak{R A}) x, x\rangle \geq 0
\end{aligned}
$$

com igualdade se e somente se cada parcela é nula, o que implica $x=0$, dado que $\mathfrak{R A}, \mathfrak{R B}$ são positivas definidas.

Terceira afirmação: $\mathcal{H}_{m}$ é aberto no subespaço de $M_{m}(\mathbb{C})$ das matrizes simétricas.

De fato, seja $A \in \mathcal{H}_{m}$. Denotemos por $\sigma(C)$ o conjunto dos autovalores de uma matriz C. Podemos escolher $\varepsilon>0$ pequeno o suficiente de forma que, para qualquer matriz 
simétrica $B$ satisfazendo $\|A-B\|<\varepsilon$ em alguma norma, tenhamos

$$
|\mu-v|<\frac{1}{2}\left(\min _{\lambda \in \sigma(\Re A)}|\lambda|\right)>0 \quad, \quad \mu \in \sigma(\Re A), v \in \sigma(\Re B)
$$

Isto se deve ao fato que as raízes complexas de um polinômio (o polinômio característico de A) variam continuamente com seus coeficientes, desde que o grau do polinômio se mantenha. Em particular, os autovalores de $\mathfrak{R B}$ são positivos, o que é equivalente a dizer que $\mathfrak{R B}$ é positiva definida.

De posse dessas três afirmações, podemos terminar a demonstração. O lema anterior nos diz que a igualdade

$$
\int_{\mathbb{R}^{m}} e^{-\langle M x, x\rangle} d x=\frac{\pi^{m / 2}}{\sqrt{\operatorname{det} M}}
$$

vale quando $M \in \mathcal{H}_{m} \cap M_{m}(\mathbb{R})$. Considere (4.4) como uma equação entre funções de $M$ definidas em $\mathcal{H}_{m}$, considerado como subconjunto de $\mathbb{C}^{\frac{\mathfrak{m}(\mathfrak{m}+1)}{2}}$ (lembremos que $\mathcal{H}_{\mathrm{m}}$ é um conjunto de matrizes simétricas ). Assim, a igualdade vale em $\mathcal{H}_{m} \cap \mathbb{R}^{\frac{\mathfrak{m}(\mathfrak{m}+1)}{2}}$. Mas podemos utilizar o ramo usual da raiz quadrada, mencionado no enunciado, para definir a função holomorfa $\sqrt{\operatorname{det}}$ em $\mathcal{H}_{\mathrm{m}}$. De fato, levando-se em conta que det assume alguns valores reais positivos no convexo $\mathcal{H}_{m}$, mas não assume o valor 0 , não poderá assumir valores reais negativos em $\mathcal{H}_{m}$. Sendo assim, ambos os membros da equação (4.4) são funções holomorfas de $M$ em $\mathcal{H}_{\mathrm{m}}$, e o princípio de identidade de funções holomorfas garante que a igualdade vale em todo o conjunto $\mathcal{H}_{m}$.

Lema 4.5. Se $\mathrm{B} \in \mathrm{M}_{\mathrm{m}}(\mathbb{R})$ é uma matriz real tal que $\|\mathrm{B}\|<1$ (a norma $\|\cdot\|$ no espaço $M_{m}(\mathbb{R})$ é a norma Euclidiana de $\mathbb{R}^{\mathrm{m}^{2}}$ ), e se definirmos

$$
A=I+i B
$$

onde I denota a matriz identidade em $M_{m}(\mathbb{R})$, então

$$
(\operatorname{det} A) \int_{\mathbb{R}^{m}} e^{-[A x]^{2}} \mathrm{dx}=\pi^{\mathrm{m} / 2}
$$

Demonstração: Note que $[A x]^{2}=\langle A x, A x\rangle=\left\langle A^{t} A x, x\right\rangle=\langle C x, x\rangle$, onde a matriz

$$
\mathrm{C}=(\mathrm{I}+\mathrm{iB})^{\mathrm{t}}(\mathrm{I}+\mathrm{iB})=\left(\mathrm{I}-\mathrm{B}^{\mathrm{t}} \mathrm{B}\right)+\mathrm{i}\left(\mathrm{B}+\mathrm{B}^{\mathrm{t}}\right)
$$

tem determinante $\operatorname{det} C=\operatorname{det} A^{t} A=(\operatorname{det} A)^{2}$ e parte real $I-B^{t} B$ positiva definida: se $x \in \mathbb{C}^{\mathrm{m}}$, então o fato de que $\|\mathrm{B}\|<1$ implica

$$
\left\langle\left(\mathrm{I}-\mathrm{B}^{\mathrm{t}} \mathrm{B}\right) \mathrm{x}, \mathrm{x}\right\rangle=|\mathrm{x}|^{2}-\left\langle\mathrm{B}^{\mathrm{t}} \mathrm{B} x, x\right\rangle=|x|^{2}-|\mathrm{B} x|^{2} \geq 0
$$

com igualdade se e somente se $x=0$. Portanto, pelo lema 4.3 aplicado à matriz $C$, temos

$$
\int_{\mathbb{R}^{m}} e^{-[A x]^{2}} d x=\int_{\mathbb{R}^{m}} e^{-\langle C x, x\rangle} d x=\pi^{\frac{\mathfrak{m}}{2}}(\operatorname{det} C)^{\frac{1}{2}}=\pi^{\frac{\mathfrak{m}}{2}}(\operatorname{det} A)^{-1}
$$

Multiplicamos ambos os lados da igualdade por $\operatorname{det} A$ para concluir a prova. 
Proposição 4.6. Seja $v: \mathbb{R}^{m} \rightarrow \mathbb{C}$ uma função de classe $\mathrm{C}^{\infty}$ com suporte contido em $\{|x| \leq R\}$, para algum $R>0$. Sejam $\phi_{j}: \mathbb{R}^{m} \rightarrow \mathbb{R}$ funções de classe $C^{\infty}$ com suporte contido em $\{|x| \leq R\}$, para $j=1, \ldots, m$, e defina

$$
Z_{j}(x)=x_{j}+i \phi_{j}(x) \quad, \quad x \in \mathbb{R}^{m}
$$

Suponha ainda que

$$
\|\mathrm{D} \Phi(x)\|<\frac{1}{2} \quad, \quad x \in \mathbb{R}^{\mathrm{m}}
$$

onde $\|\cdot\|$ é alguma norma em $M_{m}\left(\mathbb{R}^{m}\right)$ e $\Phi$ é a aplicação $\left(\phi_{1}, \ldots, \phi_{m}\right): \mathbb{R}^{m} \rightarrow \mathbb{R}^{m}$. Então as integrais

$$
\begin{aligned}
& \mathrm{I}_{\tau}=\int_{\mathbb{R}^{\mathrm{m}}} e^{-[\mathrm{DZ}(x) \cdot \xi]^{2}}\left(v\left(x+\tau^{-1 / 2} \xi\right)-v(x)\right) d \xi \\
& \mathrm{J}_{\tau}=\int_{\mathbb{R}^{\mathrm{m}}}\left(e^{-\tau\left[\mathrm{Z}(x)-\mathrm{Z}\left(x+\tau^{-1 / 2} \xi\right)\right]^{2}}-e^{-[\mathrm{DZ}(x) \cdot \xi]^{2}}\right) v\left(x+\tau^{-1 / 2} \xi\right) d \xi
\end{aligned}
$$

convergem uniformemente (em $x$ ) a zero quando $\tau \rightarrow \infty$.

Demonstração: Como $v \in \mathrm{C}_{\mathrm{c}}^{\infty}\left(\mathbb{R}^{\mathrm{m}}\right)$, existe uma constante $\mathrm{K} \geq 0$ tal que

$$
|v(x)| \leq K \quad, \quad|\nabla v(x)| \leq K \quad, \quad x \in \mathbb{R}^{m}
$$

sendo $\mathrm{K}$ independente de $x$. Assim, dados $x, \xi \in \mathbb{R}^{m}$ e $\tau>0$, existe pela Desigualdade do Valor Médio um vetor $\eta \in \mathbb{R}^{m}$ múltiplo de $\xi$ e com $|\eta| \leq|\xi|$ tal que

$$
\left|v\left(x+\tau^{-1 / 2} \xi\right)-v(x)\right| \leq\left|\nabla v\left(x+\tau^{-1 / 2} \eta\right)\right| \tau^{-1 / 2}|\xi| \leq K \tau^{-1 / 2}|\xi|
$$

A matriz derivada de $Z$ é dada por

$$
\mathrm{DZ}(x)=\mathrm{I}+\mathrm{iD} \Phi(x) \quad, \quad x \in \mathbb{R}^{\mathrm{m}}
$$

Logo podemos estimar, para todo $\xi \in \mathbb{R}^{\mathrm{m}}$ :

$$
\begin{aligned}
\left|e^{-[\mathrm{DZ}(x) \xi]^{2}}\right|=\exp \left(-\mathfrak{R}[\mathrm{DZ}(x) \xi]^{2}\right) & =\exp \left(-\mathfrak{R}\left(\xi_{1}^{2}+\cdots+\xi_{m}^{2}-|\mathrm{D} \Phi(x) \xi|^{2}\right)\right)= \\
= & \exp \left(-|\xi|^{2}+|\mathrm{D} \Phi(x) \xi|^{2}\right) \leq \exp \left(-|\xi|^{2}+\frac{1}{4}|\xi|^{2}\right)=e^{-\frac{3}{4}|\xi|^{2}}
\end{aligned}
$$

Com essas informações já podemos concluir o desejado para $\mathrm{I}_{\tau}$ :

$$
\left|I_{\tau}(x)\right| \leq \int_{\mathbb{R}^{m}}\left|e^{-[D Z(x) \xi]^{2}}\right| v\left(x+\tau^{-1 / 2} \xi\right)-v(x)\left|d \xi \leq K \tau^{-1 / 2} \int_{\mathbb{R}^{m}}\right| \xi \mid e^{-\left.\frac{3}{4}|\xi|\right|^{2}} d \xi=K^{\prime} \tau^{-1 / 2}
$$

onde a constante $K^{\prime}$ independe de $x$. Assim $I_{\tau} \rightarrow 0$ quando $\tau \rightarrow \infty$, e o limite é uniforme em $x \in \mathbb{R}^{m}$. 
$O$ resultado para $J_{\tau}$ requer mais alguns resultados preliminares. Começamos afirmando que, se $L>0$ é qualquer, então para $|\xi| \leq L$ temos

$$
\frac{1}{\tau^{-1 / 2}}\left(Z(x)-Z\left(x+\tau^{-1 / 2} \xi\right)\right) \underset{\tau \rightarrow \infty}{\longrightarrow}-D Z(x) \xi
$$

uniformemente em $x$. De fato, defina $h=\tau^{-1 / 2} \xi$ para facilitar a escrita. Note que

$$
\begin{aligned}
& \frac{1}{\tau^{-1 / 2}\left(Z(x)-Z\left(x+\tau^{-1 / 2} \xi\right)\right)+D Z(x) \xi=\frac{1}{\tau^{-1 / 2}}(x+i \Phi(x)-(x+h+i \Phi(x+h)))+}+(I+i D \Phi(x)) \xi \\
& = \\
& =\frac{1}{\tau^{-1 / 2}}(-h+i(\Phi(x)-\Phi(x+h)))+\xi+i D \Phi(x) \xi \\
& =\frac{1}{\tau^{-1 / 2}}(-h)+\xi-i\left(\frac{1}{\tau^{-1 / 2}}(\Phi(x+h)-\Phi(x))-D \Phi(x) \xi\right) \\
& =-\frac{i}{\tau^{-1 / 2}}(\Phi(x+h)-\Phi(x)-D \Phi(x) h)
\end{aligned}
$$

Assim podemos estimar

$$
\begin{aligned}
\left|\frac{1}{\tau^{-1 / 2}}\left(Z(x)-Z\left(x+\tau^{-1 / 2} \xi\right)\right)+D Z(x) \xi\right| & =\frac{1}{\tau^{-1 / 2}}|\Phi(x+h)-\Phi(x)-D \Phi(x) h| \\
& \leq \frac{1}{\tau^{-1 / 2}} \sum_{j=1}^{m}\left|\phi_{j}(x+h)-\phi_{j}(x)-\nabla \phi_{j}(x) h\right| \\
& \leq \frac{1}{\tau^{-1 / 2}} \sum_{j=1}^{m} \frac{D^{2} \phi_{j}(x+\eta)|h|^{2}}{2} \\
& \leq \frac{C}{\tau^{-1 / 2}}|h|^{2}=C \tau^{-1 / 2}|\xi|^{2} \leq C L^{2} \tau^{-1 / 2}
\end{aligned}
$$

para algum vetor $\eta$ entre 0 e $h$. Note que $\eta$ pode depender de $x$, mas como cada $\phi_{j}$ tem suporte compacto, a constante $C$ acima pode vir da limitação de $\left\|D^{2} \phi_{j}(\eta)\right\|$ de forma independente de $x$. Isto prova a afirmação.

A seguir afirmamos que

$$
\mathfrak{R}\left[\frac{1}{\tau^{-1 / 2}}\left(Z(x)-Z\left(x+\tau^{-1 / 2} \xi\right)\right)\right]^{2} \geq 0
$$


para quaisquer $x, \xi \in \mathbb{R}^{m}$ e $\tau>0$. De fato, defina mais uma vez $h=\tau^{-1 / 2} \xi$. Então

$$
\begin{aligned}
\Re\left[\frac{1}{\tau^{-1 / 2}}\right. & (Z(x)-Z(x+h))]^{2}=\Re\left[\frac{1}{\tau^{-1 / 2}}(i \Phi(x)-h-i \Phi(x+h))\right]^{2} \\
& =\Re\left[-\frac{1}{\tau^{-1 / 2}}(h+i \Phi(x+h)-i \Phi(x))\right]^{2} \\
& =\Re\left(\tau[h+i(\Phi(x+h)-\Phi(x))]^{2}\right) \\
& =\tau \sum_{j=1}^{m} \mathfrak{R}\left(h_{j}+i\left(\phi_{j}(x+h)-\phi_{j}(x)\right)\right)^{2} \\
& =\tau \sum_{j=1}^{m}\left(\left(\Re\left\{h_{j}+i\left(\phi_{j}(x+h)-\phi_{j}(x)\right)\right\}\right)^{2}-\right. \\
& =\tau \sum_{j=1}^{m}\left(h_{j}^{2}-\left(\phi_{j}(x+h)-\phi_{j}(x)\right)^{2}\right) \\
& =\tau|h|^{2}-\tau|\Phi(x+h)-\Phi(x)|^{2} \\
& \geq \tau|h|^{2}-\tau|| D \Phi(x+\eta) \|^{2}|h|^{2} \geq \tau|h|^{2}-\tau \frac{1}{4}|h|^{2}=\frac{3 \tau}{4}|h|^{2} \geq 0
\end{aligned}
$$

onde mais uma vez $\eta$ está entre 0 e $h$, mas a estimativa independe de $x$ porque estimamos $\|\mathrm{D} \Phi(x+\eta)\|$ uniformemente por $1 / 2$.

Observe que as afirmações (4.7) e (4.8) implicam que

$$
\mathfrak{R}[\mathrm{DZ}(x) \xi]^{2} \geq 0
$$

para quaisquer $x, \xi \in \mathbb{R}^{m} \operatorname{com}|\xi| \leq L$. Outra afirmação que nos será útil é a seguinte:

$$
\left|e^{-\tau\left[Z(x)-Z\left(x+\tau^{-1 / 2} \xi\right)\right]^{2}}-e^{-[D Z(x) \xi]^{2}}\right| \leq 2 e^{-\frac{3}{4}|\xi|^{2}}
$$

para quaisquer $x, \xi \in \mathbb{R}^{m}$ e qualquer $\tau>0$. Pela desigualdade triangular, basta provarmos que cada parcela

$$
\left|e^{-\tau\left[Z(x)-Z\left(x+\tau^{-1 / 2} \xi\right)\right]^{2}}\right| \quad, \quad\left|e^{-[D Z(x) \xi]^{2}}\right|
$$

fica menor que $\exp \left(-\frac{3}{4}|\xi|^{2}\right)$. Já fizemos esse cálculo para a segunda parcela no início desta demonstração, e podemos tratar a primeira analogamente:

$$
\begin{aligned}
\left|e^{-\tau\left[Z(x)-Z\left(x+\tau^{-1 / 2} \xi\right)\right]^{2}}\right| & =\exp \left(-\mathfrak{R}\left[\frac{1}{\tau^{-1 / 2}}\left(Z(x)-Z\left(x+\tau^{-1 / 2} \xi\right)\right)\right]^{2}\right) \\
& =\exp \left(\tau\left|\Phi\left(x+\tau^{-1 / 2} \xi\right)-\Phi(x)\right|^{2}-|\xi|^{2}\right) \\
& \leq \exp \left(\tau|| D \Phi\left(x+\tau^{-1 / 2} \eta\right) \|^{2}\left|\tau^{-1 / 2} \xi\right|^{2}-|\xi|^{2}\right) \\
& =\exp \left(\|\left. D \Phi\left(x+\tau^{-1 / 2} \xi\right)\right|^{2}|\xi|^{2}-|\xi|^{2}\right) \\
& \leq e^{-\frac{3}{4}|\xi|^{2}}
\end{aligned}
$$


De posse de (4.7), (4.8), (4.9), (4.10), podemos finalmente estimar $\mathrm{J}_{\tau}$. Seja $\varepsilon>0$ qualquer. Temos:

$$
\begin{aligned}
\left|J_{\tau}(x)\right| & \leq \int_{\mathbb{R}^{m}}\left|e^{-\tau\left[Z(x)-Z\left(x+\tau^{-1 / 2} \xi\right)\right]^{2}}-e^{-[D Z(x) \xi]^{2}}\right|\left|v\left(x+\tau^{-1 / 2} \xi\right)\right| d \xi \\
& \leq K \int_{\mathbb{R}^{m}}\left|e^{-\tau\left[Z(x)-Z\left(x+\tau^{-1 / 2} \xi\right)\right]^{2}}-e^{-[D Z(x) \xi]^{2}}\right| d \xi \\
& =K\left(\int_{|\xi| \leq L}+\int_{|\xi| \geq L}\right)\left|e^{-\tau\left[Z(x)-Z\left(x+\tau^{-1 / 2} \xi\right)\right]^{2}}-e^{-[D Z(x) \xi]^{2}}\right| d \xi
\end{aligned}
$$

onde $\mathrm{L}>0$ é escolhido de forma que a integral sobre $\{|\xi| \geq L\}$ fique menor que $\varepsilon / 2 \mathrm{~K}$. Podemos exigir isto porque, devido a (4.10), essa integral é menor que

$$
\int_{|\xi| \geq L} 2 e^{-\frac{3}{4}|\xi|^{2}} d \xi
$$

que tende a zero quando $\mathrm{L} \rightarrow \infty$, dado que $\int_{\mathbb{R}^{m}} \exp \left(-\frac{3}{4}|\xi|^{2}\right) \mathrm{d} \xi$ é convergente. Deve-se observar aqui que a escolha de $\mathrm{L}$ independe de $x$. Para tratar a integral sobre $\{|\xi| \leq \mathrm{L}\}$ acima, usamos inicialmente o lema 4.1 e as afirmações (4.8) e (4.9):

$$
\begin{aligned}
& \mathrm{K} \int_{|\xi| \leq \mathrm{L}} \mid e^{-\tau\left[Z(x)-Z\left(x+\tau^{-1 / 2} \xi\right)\right]^{2}}-e^{-[\mathrm{DZ}(x) \xi]^{2}} \mid \mathrm{d} \xi \leq \\
& \leq \mathrm{K} \int_{|\xi| \leq L}\left|\left[-\frac{1}{\tau^{-1 / 2}}\left(\mathrm{Z}(x)-\mathrm{Z}\left(x+\tau^{-1 / 2} \xi\right)\right)\right]^{2}-[\mathrm{DZ}(x) \xi]^{2}\right| \mathrm{d} \xi
\end{aligned}
$$

Escolha $\tau$ grande o bastante de forma que o integrando fique menor que $(\varepsilon / 2 K)\left(\int_{|\xi| \leq L} d \xi\right)^{-1}$. Isto pode ser feito devido a (4.7). Note ainda que este valor de $\tau$ depende apenas de $L$, e não de $x$. Dessa forma,

$$
\left|J_{\tau}(x)\right| \leq K \frac{\varepsilon}{2 K}\left(\int_{|\xi| \leq L} d \xi\right)^{-1}\left(\int_{|\xi| \leq L} d \xi\right)+K \frac{\varepsilon}{2 K}=\varepsilon
$$

exatamente o que precisávamos provar.

Corolário 4.11. Sejam $u: \mathbb{R}^{m} \rightarrow \mathbb{C}$ uma função de classe $C^{\infty}$ e $h: \mathbb{R}^{m} \rightarrow \mathbb{R}$ uma função de corte de classe $C^{\infty}$ que vale 1 em $\{|x| \leq R / 2\}$ e se anula para $|x| \geq R$, para algum $\mathrm{R}>0$. Sejam $\mathrm{Z}$ e $\Phi=\left(\phi_{1}, \ldots, \phi_{\mathrm{m}}\right)$ como na proposição anterior. Então a integral

$$
\mathrm{G}_{\tau}(x)=\left(\frac{\tau}{\pi}\right)^{\mathrm{m} / 2} \int_{\mathbb{R}^{\mathrm{m}}} e^{-\tau[Z(x)-Z(\xi)]^{2}} u(\xi) h(\xi)(\operatorname{det} \mathrm{DZ}(\xi)) \mathrm{d} \xi
$$

converge a $\mathrm{u}(\mathrm{x}) \mathrm{h}(\mathrm{x})$, quando $\tau \rightarrow \infty$, uniformemente em $\mathrm{x} \in \mathbb{R}^{\mathrm{m}}$. Em particular, para $|x| \leq R / 2$, o limite é $u(x)$. 
Demonstração: Como $\mathrm{DZ}(x)=\mathrm{I}+\mathrm{iD} \Phi(x)$ e $\|\mathrm{D} \Phi(x)\|<1 / 2$ para todo $x \in \mathbb{R}^{\mathrm{m}}$, o lema 4.5 garante que

$$
(\operatorname{det} \mathrm{DZ}(\mathrm{x})) \int_{\mathbb{R}^{\mathrm{m}}} e^{-[\mathrm{DZ}(\mathrm{x}) \xi]^{2}} \mathrm{~d} \xi=\pi^{\frac{\mathrm{m}}{2}}
$$

Defina uma função $v \in \mathrm{C}_{\mathrm{c}}^{\infty}\left(\mathbb{R}^{\mathrm{m}}\right)$, suportada em $|x| \leq \mathrm{R}$, por

$$
v(x)=u(x) h(x) \operatorname{det} D Z(x)
$$

Dessa forma,

$$
\pi^{-\frac{\mathfrak{m}}{2}} \int_{\mathbb{R}^{m}} e^{-[\mathrm{DZ}(x) \xi]^{2}} v(x) \mathrm{d} \xi=v(x) \pi^{-\frac{\mathfrak{m}}{2}} \int_{\mathbb{R}^{m}} e^{-[\mathrm{DZ}(x) \xi]^{2}} \mathrm{~d} \xi=v(x)(\operatorname{det} \mathrm{DZ}(x))^{-1}=h(x) u(x)
$$

Através da mudança de variável $\xi=\tau^{-1 / 2} \eta-\chi$, podemos escrever

$$
\begin{aligned}
G_{\tau}(x) & =\left(\frac{\tau}{\pi}\right)^{m / 2} \int_{\mathbb{R}^{m}} e^{-\tau[Z(x)-Z(\eta)]^{2}} u(\eta) h(\eta)(\operatorname{det} D Z(\eta)) d \eta \\
& =\left(\frac{\tau}{\pi}\right)^{m / 2} \int_{\mathbb{R}^{m}} e^{-\tau[Z(x)-Z(\eta)]^{2}} v(\eta) d \eta \\
& =\pi^{-\frac{m}{2}} \int_{\mathbb{R}^{m}} e^{-\tau\left[Z(x)-Z\left(x+\tau^{-1 / 2} \xi\right)\right]^{2}} v\left(x+\tau^{-1 / 2} \xi\right) d \xi
\end{aligned}
$$

e portanto

$$
\begin{aligned}
\mathrm{G}_{\tau}(x) & -h(x) u(x)= \\
& =\pi^{-\frac{\mathfrak{m}}{2}}\left(\int_{\mathbb{R}^{m}} e^{-\tau\left[Z(x)-Z\left(x+\tau^{-1 / 2} \xi\right)\right]^{2}} v\left(x+\tau^{-1 / 2} \xi\right) d \xi-\int_{\mathbb{R}^{m}} e^{-[D Z(x) \xi]^{2}} v(x) d \xi\right) \\
& =\pi^{-\frac{m}{2}}\left(I_{\tau}(x)-J_{\tau}(x)\right)
\end{aligned}
$$

com a notação da proposição anterior. Concluímos imediatamente que $G_{\tau}(x) \rightarrow h(x) u(x)$ uniformemente em $x$.

Corolário 4.12. Sejam $u: \mathbb{R}^{m} \times \mathbb{R}^{n} \rightarrow \mathbb{C}$ uma função de classe $C^{\infty}$ e $\mathrm{h}: \mathbb{R}^{\mathrm{m}} \rightarrow \mathbb{R}$ uma função de corte de classe $\mathrm{C}^{\infty}$ que vale 1 em $\{|x| \leq R / 2\}$ e se anula para $|x| \geq R$, para algum $\mathrm{R}>0$. Sejam $\phi_{j}: \mathbb{R}^{m} \times \mathbb{R}^{n} \rightarrow \mathbb{R}$ funções de classe $\mathrm{C}^{\infty}$ com suporte contido em $\left\{(x, t) \in \mathbb{R}^{m} \times \mathbb{R}^{n} ;|x| \leq R,|t| \leq R\right\}$, para $j=1, \ldots, m$, e defina

$$
Z_{j}(x, t)=x_{j}+i \phi_{j}(x, t) \quad, \quad x \in \mathbb{R}^{m}, t \in \mathbb{R}^{n}
$$

Suponha ainda que

$$
\left\|\frac{\partial \Phi}{\partial x}(x, t)\right\|<\frac{1}{2} \quad, \quad x \in \mathbb{R}^{m}, t \in \mathbb{R}^{n}
$$

onde $\|\cdot\|$ é alguma norma em $M_{m}\left(\mathbb{R}^{m}\right)$, $\Phi$ é a aplicação $\left(\phi_{1}, \ldots, \phi_{m}\right): \mathbb{R}^{m} \times \mathbb{R}^{n} \rightarrow \mathbb{R}^{m}$, e $\partial_{x} \Phi$ é a matriz $\left(\partial_{x_{j}} \phi_{k}\right)_{k, j}$. Então a integral

$$
\mathrm{G}_{\tau}(x, \mathrm{t})=\left(\frac{\tau}{\pi}\right)^{\mathrm{m} / 2} \int_{\mathbb{R}^{\mathrm{m}}} e^{-\tau[Z(x, t)-Z(\xi, t)]^{2}} \mathrm{u}(\xi, t) h(\xi) \operatorname{det} \frac{\partial Z}{\partial x}(\xi, t) d \xi
$$

converge a $\mathrm{u}(\mathrm{x}, \mathrm{t})$, quando $\tau \rightarrow \infty$, uniformemente no conjunto $\{|x|<\mathrm{R} / 2,|\mathrm{t}|<\mathrm{C}\}$, onde $\mathrm{C}>0$ é qualquer constante. 
Demonstração: A diferença com relação à proposição 4.6 e ao corolário 4.11 foi a adição das variáveis $t \in \mathbb{R}^{n}$ como parâmetros. Basta reproduzirmos as mesmas demonstrações, mas agora, para limitarmos $|v|$ e $|\nabla v|$ uniformemente com relação a $t$, devemos nos restringir a algum compacto $\{|\boldsymbol{t}| \leq \mathrm{C}\}$.

A partir de agora, fixe uma estrutura localmente integrável $\mathcal{V}$ em $\mathbb{R}^{N}=\{(x, t) ; x \in$ $\mathbb{R}^{m}, t \in \mathbb{R}^{n}$ \} gerada globalmente por campos da forma

$$
L_{j}=\frac{\partial}{\partial t_{j}}-i \sum_{k=1}^{m} \frac{\partial \phi_{k}}{\partial t_{j}}(x, t) M_{k} \quad, \quad j=1, \ldots, n
$$

onde

$$
M_{k}=\sum_{l=1}^{m} \mu_{k l}(x, t) \frac{\partial}{\partial x_{l}}
$$

são campos satisfazendo $M_{k} Z_{l}=\delta_{k l}$ (Delta de Kronecker) e $Z=\left(Z_{1}, \ldots, Z_{m}\right.$ ) é uma família global de integrais primeiras para $\mathcal{V}$, escritas na forma

$$
Z_{k}(x, t)=x_{k}+i \phi_{k}(x, t) \quad, \quad k=1, \ldots, m
$$

com $\phi_{1}, \ldots, \phi_{m} \in C^{\infty}\left(\mathbb{R}^{m}\right)$ a valores reais e com suporte contido em $\{|x| \leq R,|t| \leq R\}$, para algum $R>0$. Suponha ainda que a aplicação $\Phi=\left(\phi_{1}, \ldots, \phi_{m}\right)$ satisfaz

$$
\left\|\frac{\partial \Phi}{\partial x}(x, t)\right\|<\frac{1}{2} \quad, \quad x \in \mathbb{R}^{m}, t \in \mathbb{R}^{n}
$$

De acordo com o estudado no início deste trabalho, sempre podemos assumir, através de uma mudança de coordenadas, que uma dada estrutura localmente integrável satisfaz as propriedades acima localmente. Entretanto, como estamos fazendo um estudo pontual (local) de uma estrutura localmente integrável, podemos primeiramente encontrar, em alguma vizinhança $W$ do ponto $p$ em questão, o sistema de coordenadas que fornece as propriedades acima, e então modificar a estrutura fora de $W$, de forma que as propriedades sejam válidas globalmente. Essa modificação pode ser levada a cabo da seguinte maneira:

- Utilizando funções de corte suaves, suportadas em algum aberto $\mathrm{U} \supseteq \mathrm{W}$ em torno de $p$, e iguais a 1 em $W$, podemos estender $\Phi$ a todo o $\mathbb{R}^{N}$. Também é possível fazê-lo mantendo a limitação de seu Jacobiano nas variáveis $\chi$.

- Agora as funções $Z_{k}$ estão definidas globalmente. A estrutura $\mathcal{V}$ pode ser redefinida como o anulador das $Z_{k}$. Desta forma, está definida globalmente, coincide com a estrutura original em $W$, e ainda tem $\left\{Z_{k}\right\}_{k=1, \ldots, m}$ como uma família de integrais primeiras.

- Os campos $M_{k}$, caracterizados por $M_{k} Z_{l}=\delta_{k l}$, podem ser definidos inicialmente apenas em alguma vizinhança Ũ de $p$, pois são obtidos tal como no teorema 1.54 . 
Mas, conforme a definição de $Z$, é simples defini-los fora de $U$, onde as $\phi_{j}$ estão suportadas: basta tomar $M_{k}=\partial / \partial x_{k}$. Assim, diminuindo $U$ se necessário (para que $\mathrm{U} \subseteq \tilde{\mathrm{U}})$, a definição dos $M_{\mathrm{k}}$ é global.

- Finalmente, a expressão $L_{j}=\partial / \partial t_{j}-i \sum_{k=1}^{m}\left(\partial \phi_{k} / \partial t_{j}\right)(x, t) M_{k}$ define os campos $L_{j}$ globalmente, e eles geram a estrutura devido ao teorema 1.54.

Será conveniente notar que existem funções suaves $\lambda_{j k}(x, t), j=1, \ldots, n, k=1, \ldots, m$, tais que

$$
L_{j}=\frac{\partial}{\partial t_{j}}+\sum_{k=1}^{m} \lambda_{j k}(x, t) \frac{\partial}{\partial x_{k}} \quad, \quad j=1, \ldots, n
$$

De fato, basta escrever por extenso os campos $M_{k}$ na definição de $L_{j}$, e então escrever a expressão resultante como uma combinação $C^{\infty}$-linear dos campos $\partial / \partial t_{j}, \partial / \partial x_{k}$.

Fixe também $h: \mathbb{R}^{m} \rightarrow \mathbb{R}$ função de corte de classe $C^{\infty}$ que vale 1 em $\{|x| \leq R / 2\}$ e se anula para $|x| \geq R$. Dada qualquer função $v: \mathbb{R}^{N} \rightarrow \mathbb{C} \in C^{\infty}\left(\mathbb{R}^{N}\right)$, definimos as integrais:

$$
\begin{aligned}
& E_{\tau}(v)(x, t)=\left(\frac{\tau}{\pi}\right)^{m / 2} \int_{\mathbb{R}^{m}} e^{-\tau[Z(x, t)-Z(\xi, 0)]^{2}} v(\xi, 0) h(\xi) \operatorname{det} \frac{\partial Z}{\partial x}(\xi, 0) d \xi \\
& G_{\tau}(v)(x, t)=\left(\frac{\tau}{\pi}\right)^{m / 2} \int_{\mathbb{R}^{m}} e^{-\tau[Z(x, t)-Z(\xi, t)]^{2}} v(\xi, t) h(\xi) \operatorname{det} \frac{\partial Z}{\partial x}(\xi, t) d \xi
\end{aligned}
$$

Teorema 4.13. Nas condições acima, existe $\mathrm{T}>0$ tal que, para toda $u \in \mathrm{C}^{\infty}\left(\mathbb{R}^{\mathrm{N}}\right)$ solução de $\mathcal{V}$,

$$
\mathrm{G}_{\tau}(\mathrm{u})(x, \mathrm{t})-\mathrm{E}_{\tau}(\mathrm{u})(x, \mathrm{t}) \underset{\tau \rightarrow \infty}{\longrightarrow} 0
$$

uniformemente no conjunto

$$
\left\{|x| \leq \frac{R}{4},|t| \leq T\right\}
$$

Demonstração: Como as formas $d Z_{1}, \ldots, d Z_{m}, d t_{1}, \ldots, d t_{n}$ são linearmente independentes em qualquer ponto $(x, t)$, devem gerar $\mathbb{C T}_{(x, t)}^{*} \mathbb{R}^{N}$. E como os campos $M_{1}, \ldots, M_{m}, L_{1}, \ldots$, $L_{n}$ são os seus respectivos duais, uma função qualquer $g \in C^{\infty}\left(\mathbb{R}^{N}\right)$ terá sua diferencial escrita na forma

$$
d g=\sum_{j=1}^{n} L_{j} g d t_{j}+\sum_{k=1}^{m} M_{k} g d Z_{k}
$$

Seja $T \in(0,1)$ inicialmente arbitrário. Fixe $(x, t) \in \mathbb{R}^{N}$, com $|x| \leq R / 4$ e $|t| \leq T$. Seja $C>0$ tal que

$$
\left|\Phi\left(\xi, \rho_{1}\right)-\Phi\left(\xi, \rho_{2}\right)\right| \leq C\left|\rho_{1}-\rho_{2}\right| \quad, \quad|\xi| \leq R,\left|\rho_{1}\right|,\left|\rho_{2}\right| \leq 1
$$


$C$ pode ser tomado independente de $(x, t)$ sob as restrições que estamos assumindo para esse ponto, e também independe de T. De fato, pela Desigualdade do Valor Médio, é suficiente tomar

$$
C=\max _{k=1, \ldots, m} \max _{j=1, \ldots, n} \sup _{|\xi| \leq R,|\rho| \leq 1}\left|\frac{\partial \phi_{k}}{\partial t_{j}}(\xi, \rho)\right|
$$

e então corrigir $C$ por um fator que leve em conta a equivalência entre as normas Euclidiana e da soma em $\mathbb{R}^{n}$.

Defina

$$
\begin{aligned}
g(\xi, \rho) & =\left(\frac{\tau}{\pi}\right)^{m / 2} e^{-\tau[Z(x, t)-Z(\xi, \rho)]^{2}} u(\xi, \rho) h(\xi) \\
\omega(\xi, \rho) & =g(\xi, \rho) d Z(\xi, \rho)
\end{aligned}
$$

para $\xi \in \mathbb{R}^{m}$ e $\rho \in \mathbb{R}^{n}$. Note que

$$
\int_{\mathbb{R}^{m} \times\{0\}} \omega(\xi, \rho)=E_{\tau}(u)(x, t) \quad, \quad \int_{\mathbb{R}^{m} \times\{t\}} \omega(\xi, \rho)=G_{\tau}(u)(x, t)
$$

já que o pullback de $\omega$ a um conjunto da forma $\mathbb{R}^{m} \times\left\{t_{0}\right\}$ é

$$
\begin{aligned}
&\left(\frac{\tau}{\pi}\right)^{m / 2} e^{-\tau\left[Z(x, t)-Z\left(\xi, t_{0}\right)\right]^{2}} u\left(\xi, t_{0}\right) h(\xi) d Z\left(\xi, t_{0}\right)= \\
&=\left(\frac{\tau}{\pi}\right)^{m / 2} e^{-\tau\left[Z(x, t)-Z\left(\xi, t_{0}\right)\right]^{2}} u\left(\xi, t_{0}\right) h(\xi) \operatorname{det} \frac{\partial Z}{\partial x}\left(\xi, t_{0}\right) d \xi
\end{aligned}
$$

A fronteira da variedade $\mathbb{R}^{m} \times[0, t]$, onde $[0, t]$ denota o segmento de reta entre 0 e $t$, é dada por $\mathbb{R}^{\mathrm{m}} \times\{\mathrm{t}\}-\mathbb{R}^{\mathrm{m}} \times\{0\}$ (indicamos com o sinal de menos o fato que a orientação é negativa na segunda componente). Assim, o Teorema de Stokes implica que

$$
G_{\tau}(x, t)-E_{\tau}(x, t)=\int_{\mathbb{R}^{m} \times\{t\}} \omega(\xi, \rho)-\int_{\mathbb{R}^{m} \times\{0\}} \omega(\xi, \rho)=\int_{\mathbb{R}^{m} \times[0, t]} d \omega(\xi, \rho)
$$

onde, pela equação (4.14),

$$
d \omega=d g \wedge d Z=\left(\sum_{j=1}^{n}\left(L_{j} g\right) d t_{j} \wedge d Z\right)+\left(\sum_{k=1}^{m}\left(M_{k} g\right) d Z_{j} \wedge d Z\right)=\sum_{j=1}^{n}\left(L_{j} g\right) d t_{j} \wedge d Z
$$

Calculamos $\mathrm{L}_{j} \mathrm{~g}=\mathrm{L}_{\mathrm{j}} \mathrm{g}(\xi, \rho)$ pela Regra de Leibniz, observando que $\mathrm{L}_{\mathrm{j}} \mathrm{u}(\xi, \rho)=0$ e $\mathrm{L}_{j} \exp \left(-\tau[Z(x, t)-Z(\xi, \rho)]^{2}\right)=0$, esta última por se tratar de uma função holomorfa composta com $Z(\xi, \rho)$. Então,

$$
\mathrm{L}_{j} g(\xi, \rho)=\left(\frac{\tau}{\pi}\right)^{\mathrm{m} / 2} e^{-\tau[Z(x, t)-Z(\xi, \rho)]^{2}} u(\xi, \rho) \mathrm{L}_{j} h(\xi)
$$

Segue que

$$
G_{\tau}(x, t)-E_{\tau}(x, t)=\left(\frac{\tau}{\pi}\right)^{m / 2} \sum_{j=1}^{n} \int_{\mathbb{R}^{m} \times[0, t]} e^{-\tau[Z(x, t)-Z(\xi, \rho)]^{2}} u(\xi, \rho) L_{j} h(\xi) d t_{j} \wedge d Z(\xi, \rho)
$$


Como $h$ é constante em $\{|\xi|<R / 2\}$ e em $\{|\xi|>R\}$, a integral acima pode ser tomada em $\{R / 2 \leq|\xi| \leq R\} \times[0, t]$. Mas nesse compacto, podemos limitar a norma de $u(\xi, \rho) L_{j} h(\xi)$ por uma constante $K \geq 0$, que independe de $x$ e de $t$, uma vez que estamos considerando $|\mathrm{t}| \leq \mathrm{T}$. Também incorporaremos o fator $\pi^{-\mathrm{m} / 2}$ em $\mathrm{K}$, para escrever

$$
\left|G_{\tau}(x, t)-E_{\tau}(x, t)\right| \leq K \tau^{m / 2} \sum_{j=1}^{n} \int_{\{R / 2 \leq|\xi| \leq R\} \times[0, t]}\left|e^{-\tau[Z(x, t)-Z(\xi, \rho)]^{2}}\right|\left|d t_{j} \wedge d Z(\xi, \rho)\right|
$$

Calculemos o módulo que aparece no integrando:

$$
\begin{aligned}
\left|e^{-\tau[Z(x, t)-Z(\xi, \rho)]^{2}}\right| & =\exp \Re\left(-\tau[Z(x, t)-Z(\xi, \rho)]^{2}\right) \\
& =\exp \Re\left(-\tau[x-\xi+i(\Phi(x, t)-\Phi(\xi, \rho))]^{2}\right) \\
& =\exp \left(-\tau \Re \sum_{j=1}^{m}\left(x_{j}-\xi_{j}+i\left(\phi_{j}(x, t)-\phi_{j}(\xi, \rho)\right)\right)^{2}\right) \\
& =\exp \left(-\tau \sum_{j=1}^{m}\left(\left(x_{j}-\xi_{j}\right)^{2}-(\Phi(x, t)-\Phi(\xi, \rho))^{2}\right)\right) \\
& =\exp \left(-\tau|x-\xi|^{2}+\tau|\Phi(x, t)-\Phi(\xi, \rho)|^{2}\right)
\end{aligned}
$$

Dado que $|\xi| \geq R / 2$ e $\rho \in[0, t]$, podemos ainda estimar

$$
\begin{aligned}
|\Phi(x, t)-\Phi(\xi, \rho)| & \leq|\Phi(x, t)-\Phi(\xi, t)|+|\Phi(\xi, t)-\Phi(\xi, \rho)| \\
& \leq \frac{1}{2}|x-\xi|+C|t-\rho| \\
& \leq \frac{1}{2}|x-\xi|+2 C T
\end{aligned}
$$

Então,

$$
|\Phi(x, t)-\Phi(\xi, \rho)|^{2} \leq \frac{1}{4}|x-\xi|^{2}+2|x-\xi| C T+4 C^{2} T^{2} \leq \frac{1}{2}|x-\xi|^{2}+8 C^{2} T^{2}
$$

Nesta última passagem, usamos a desigualdade entre as médias aritmética e geométrica tal como segue:

$$
\begin{aligned}
2|x-\xi| C T=\frac{|x-\xi|}{\sqrt{2}}(\sqrt{8} \mathrm{CT})=\sqrt{\frac{|x-\xi|^{2}}{2}\left(8 \mathrm{C}^{2} \mathrm{~T}^{2}\right)} & \leq \\
& \leq \frac{1}{2}\left(\frac{|x-\xi|^{2}}{2}+8 \mathrm{C}^{2} \mathrm{~T}^{2}\right)=\frac{|x-\xi|^{2}}{4}+4 \mathrm{C}^{2} \mathrm{~T}^{2}
\end{aligned}
$$

Voltando a (4.16), ficamos com

$$
\begin{aligned}
\left|e^{-\tau[Z(x, t)-Z(\xi, \rho)]^{2}}\right|=\exp \left(-\tau|x-\xi|^{2}+\tau|\Phi(x, t)-\Phi(\xi, \rho)|^{2}\right) & \leq \\
& \leq \exp \left(8 \tau C^{2} T^{2}-\frac{\tau|x-\xi|^{2}}{2}\right) \leq \exp \left(\tau\left(8 C^{2} T^{2}-\frac{R^{2}}{32}\right)\right)
\end{aligned}
$$


O que usamos aqui foi o fato que, como $|\xi| \geq R / 2$ e $|x| \leq R / 4$, temos $|x-\xi| \geq R / 4$. Então, se $T>0$ for escolhido pequeno o suficiente para que $8 C^{2} T^{2}<R^{2} / 32$, obtemos

$$
\left|e^{-\tau[Z(x, t)-Z(\xi, \rho)]^{2}}\right|<e^{-\frac{\tau R^{2}}{64}}
$$

Substituindo em (4.15),

$$
\begin{aligned}
\left|G_{\tau}(x, t)-E_{\tau}(x, t)\right| \leq K \tau^{m / 2} \sum_{j=1}^{n} \int_{\{R / 2 \leq|\xi| \leq R\} \times[0, t]} e^{-\frac{\tau R^{2}}{64}}\left|d t_{j} \wedge d Z(\xi, \rho)\right| \leq \\
\quad \leq K \tau^{m / 2} e^{-\frac{\tau R^{2}}{64}} \sum_{j=1}^{n} \int_{\{R / 2 \leq|\xi| \leq R\} \times[0, T]}\left|d t_{j} \wedge d Z(\xi, \rho)\right|
\end{aligned}
$$

que, conforme queríamos demonstrar, converge a 0 uniformemente em $(x, t)$ quando $\tau \rightarrow$ $\infty$.

Teorema 4.17. (Fórmula de Aproximação de Baouendi-Trèves) Sejam $\Omega$ uma variedade $\mathrm{N}$-dimensional, $\mathcal{V}$ uma estrutura localmente integrável de posto $\mathrm{n}$ em $\Omega$, e $\mathrm{Z}=\left(\mathrm{Z}_{1}, \ldots, \mathrm{Z}_{\mathrm{m}}\right)$ um sistema de integrais primeiras para $\mathcal{V}$ em torno de um ponto $\mathrm{p} \in \Omega$. Então existem abertos $\mathrm{U}, \mathrm{W} \ni \mathrm{p}$, com $\mathrm{U} \subset \subset \mathrm{W} \subset \subset \Omega$, tais que, para toda $\mathrm{u} \in \mathrm{C}^{\infty}(\mathrm{W})$, se $\mathrm{Vu}=0 \mathrm{em} \mathrm{W}$ então u é limite uniforme em $\mathrm{U}$ de polinômios nas funções $Z_{k}$.

Demonstração: Podemos e vamos assumir que $\mathcal{V}$ e $Z$ são dadas como nos resultados acima, com $\Omega \subseteq \mathbb{R}^{N}$ e $p=0$. Tomamos

$$
U=\left\{|x| \leq \frac{R}{4},|t| \leq T\right\} \quad, \quad W=\left\{|x| \leq \frac{R}{2},|t| \leq R\right\}
$$

onde R é o mesmo com o qual estamos trabalhando até agora e T é obtido como no teorema 4.13. (Se necessário, diminua $T$ para que $U \subset \subset W$ ). Seja $u \in C^{\infty}(W)$ uma solução da estrutura. Tome ainda uma função de corte $h$ suportada em $\{|x| \leq R\}$ e valendo 1 em $\{|x| \leq R / 2\}$. O corolário 4.11 afirma que

$$
\mathrm{G}_{\tau}(\mathrm{u})(x, \mathrm{t})=\left(\frac{\tau}{\pi}\right)^{\mathrm{m} / 2} \int_{\mathbb{R}^{m}} e^{-\tau[Z(x, t)-Z(\xi, t)]^{2}} u(\xi, t) h(\xi) \operatorname{det} \frac{\partial Z}{\partial x}(\xi, t) d \xi
$$

converge a $u(x, t)$ quando $\tau \rightarrow \infty$, uniformemente em $(x, t) \in W$ (e isso ocorre independentemente de u ser ou não solução da estrutura). Já o teorema 4.13 prova que, se

$$
E_{\tau}(u)(x, t)=\left(\frac{\tau}{\pi}\right)^{m / 2} \int_{\mathbb{R}^{m}} e^{-\tau[Z(x, t)-Z(\xi, 0)]^{2}} u(\xi, 0) h(\xi) \operatorname{det} \frac{\partial Z}{\partial x}(\xi, 0) d \xi
$$

então $G_{\tau}(x, t)-E_{\tau}(x, t)$ converge a 0 quando $\tau \rightarrow \infty$, uniformemente em $(x, t) \in U$. Em particular $E_{\tau}(u)(x, t)$ deve convergir uniformemente a $u$. Assim, o espaço

$$
\mathcal{E}_{2}=\left\{\mathrm{E}_{\tau}(\mathrm{u}) ; \tau>0, u \in \mathrm{C}^{\infty}(\mathrm{W}), \nu \mathrm{u}=0 \mathrm{em} \mathrm{u}\right\}
$$


é denso no espaço

$$
\mathcal{E}_{1}=\left\{u ; u \in C^{\infty}(W), v u=0 \text { em } u\right\}
$$

ambos com a topologia da convergência uniforme.

Para cada $k \in \mathbb{N}$ e cada $\tau>0$, denote por $P_{k, \tau}(\zeta)$ o Polinômio de Taylor de grau $k$ da função inteira $e^{-\tau[\zeta]^{2}}, \zeta \in \mathbb{C}^{\mathrm{m}}$, com centro na origem, definido em algum disco compacto contendo o conjunto $\{(Z(x, t)-Z(\xi, 0)) ;(x, t) \in U,|\xi| \leq R\}$. Observe que

$$
\mathrm{Q}(x, \mathrm{t})=\left(\frac{\tau}{\pi}\right)^{\mathrm{m} / 2} \int_{\mathbb{R}^{\mathrm{m}}} \mathrm{P}_{k, \tau}(Z(x, t)-Z(\xi, 0)) \mathrm{u}(\xi, 0) h(\xi) \operatorname{det} \frac{\partial Z}{\partial x}(\xi, 0) d \xi
$$

é um polinômio em $Z_{1}(x, t), \ldots, Z_{m}(x, t)$, porque a integral é feita com relação a $\xi$. Também, para cada $\tau$ fixo,

$$
Q_{k, \tau}(x, t) \underset{k \rightarrow \infty}{\longrightarrow} E_{\tau}(x, t)
$$

uniformemente em $(x, t) \in U$ (mais do que isso, em norma $C^{\infty}$ ). Então o espaço

$$
\mathcal{E}_{3}=\left\{\mathrm{P}\left(\mathrm{Z}_{1}, \ldots, \mathrm{Z}_{\mathrm{m}}\right) ; \mathrm{P} \text { é um polinômio }\right\}
$$

é denso em $\mathcal{E}_{2}$ com a topologia da convergência uniforme. Como a densidade de subespaços topológicos é transitiva, isto é suficiente para garantir que $\mathcal{E}_{3}$ é denso em $\mathcal{E}_{1}$, que é equivalente ao que queríamos mostrar.

Observação 4.18. Vale ressaltar aqui que a Fórmula de Aproximação também pode ser usada para concluir o princípio da constância local (com a fórmula da maneira como foi provada aqui, provamos o princípio apenas para soluções suaves), mas agora para estruturas de coposto arbitrário. De fato, se u é solução suave de $\mathcal{V}$, então, no aberto $\mathrm{U} \ni \mathrm{p}$ do teorema, $\mathrm{u}$ é limite pontual de polinômios nas $\mathrm{Z}_{\mathrm{k}}$, o que implica que u é constante nas fibras da aplicação $\mathrm{Z}=\left(\mathrm{Z}_{1}, \ldots, \mathrm{Z}_{\mathrm{m}}\right)$. Pelo mesmo resultado de Topologia utilizado na demonstração do teorema 2.13, podemos concluir que existe ũ continua em Z(U) tal que $\mathrm{u}=\tilde{u} \circ \mathrm{Z}$ em $\mathrm{U}$.

Exemplo 4.19. $E m \mathbb{R}^{2}=\{(t, x)\}$, considere a estrutura gerada pelo operador de CauchyRiemann:

$$
\frac{\partial}{\partial \bar{\zeta}}=\frac{1}{2}\left(\frac{\partial}{\partial t}+i \frac{\partial}{\partial x}\right)
$$

Sabemos que suas soluções são as funções holomorfas da variável complexa $\zeta=\mathrm{t}+\mathrm{ix}$, que é uma integral primeira global da estrutura. Então a Fórmula de Aproximação prova que funções holomorfas são limites uniformes locais de polinômios em $\zeta$.

Exemplo 4.20. $E m \mathbb{R}^{n+1}=\left\{\left(t_{1}, \ldots, t_{n}, x\right)\right\}$, considere a estrutura gerada pela derivada $\partial_{x}$, cujas soluções continuas são da forma $\mathrm{u}(\mathrm{t}, \mathrm{x})=\mathrm{f}(\mathrm{t})$, para $\mathrm{f}$ continua. Uma possivel familia de integrais primeiras globais nesse caso é $\mathrm{Z}(\mathrm{t}, \mathrm{x})=\left(\mathrm{t}_{1}, \ldots, \mathrm{t}_{\mathrm{n}}\right)$, e então a Fórmula de Aproximação prova que funções continuas em $\mathbb{R}^{n}$ são limites uniformes locais de polinômios nas $n$ variáveis do espaço euclidiano. 
Agora abordaremos a questão da convergência da sequência de polinômios em $Z$ obtida no teorema acima. Por enquanto, sabemos que ela se dá no espaço das funções contínuas. Mas vale algo mais forte que isso: a convergência em $C^{\infty}$, isto é, convergência uniforme de todas as derivadas. O trabalho para provar isso já está quase pronto. Precisaremos apenas provar resultados a respeito do comutador entre os operadores $L_{j}, M_{k}$ e as aplicações $G_{\tau}, E_{\tau}$.

Lema 4.21. Se $\mathrm{t} \in \mathbb{R}^{n}$ e $u \in \mathrm{C}^{\infty}\left(\mathbb{R}^{\mathrm{N}}\right)$ são quaisquer, então

$$
\int_{\mathbb{R}^{m} \times\{t\}}\left(M_{k} u\right) d Z=0, \quad k=1, \ldots, m
$$

Demonstração: Fixado $k=1, \ldots, m$, introduza a forma diferencial exata

$$
\begin{aligned}
w_{k} & =d\left(u d Z_{1} \wedge \cdots \wedge d Z_{k-1} \wedge d Z_{k+1} \wedge \cdots \wedge d Z_{m}\right) \\
& =d u \wedge d Z_{1} \wedge \cdots \wedge d Z_{k-1} \wedge d Z_{k+1} \wedge \cdots \wedge d Z_{m} \\
& =\left(\sum_{j=1}^{n} L_{j} u d t_{j}+\sum_{p=1}^{m} M_{p} u d Z_{p}\right) \wedge d Z_{1} \wedge \cdots \wedge d Z_{k-1} \wedge \\
& =\left(\sum_{j=1}^{n} L_{j} u d t_{j}+M_{k} u d Z_{k}\right) \wedge d Z_{k+1} \wedge \cdots \wedge d_{d Z_{m}=} \wedge Z_{k-1} \wedge d Z_{k+1} \wedge \cdots \wedge d Z_{m}= \\
& =\sum_{j=1}^{n} L_{j} u d t_{j} \wedge d Z_{1} \wedge \cdots \wedge d Z_{k-1} \wedge d Z_{k+1} \wedge \cdots \wedge d Z_{m}+(-1)^{k-1} M_{k} u d Z
\end{aligned}
$$

O pullback de $\omega_{k}$ a $\mathbb{R}^{m} \times\{t\}$ é portanto dado por $(-1)^{k-1} M_{k} u d Z$. Sendo $\omega_{k}$ exata, esse pullback é também exato, logo $0=\int_{\mathbb{R}^{\mathrm{m}} \times\{\mathrm{t}\}} \omega_{\mathrm{k}}=(-1)^{\mathrm{k}-1} \int_{\mathbb{R}^{\mathrm{m}} \times\{\mathrm{t}\}} M_{\mathrm{k}} \mathrm{udZ}$.

Lema 4.22. Se $u \in C^{\infty}\left(\mathbb{R}^{N}\right)$ é qualquer, então valem as fórmulas:

$$
\begin{aligned}
M_{k} G_{\tau}(u)(x, t)-G_{\tau}\left(M_{k} u\right)(x, t) & = \\
= & \left(\frac{\tau}{\pi}\right)^{m / 2} \int_{\mathbb{R}^{m}} e^{-\tau[Z(x, t)-Z(\xi, t)]^{2}} u(\xi, t) M_{k} h(\xi) \operatorname{det} \frac{\partial Z}{\partial x}(\xi, t) d \xi \\
M_{k} E_{\tau}(u)(x, t)-E_{\tau}\left(M_{k} u\right)(x, t) & = \\
= & \left(\frac{\tau}{\pi}\right)^{m / 2} \int_{\mathbb{R}^{m}} e^{-\tau[Z(x, t)-Z(\xi, 0)]^{2}} u(\xi, 0) M_{k} h(\xi) \operatorname{det} \frac{\partial Z}{\partial x}(\xi, 0) d \xi
\end{aligned}
$$

para todo $\mathrm{k}=1, \ldots, \mathrm{m}$ e todo $(\mathrm{x}, \mathrm{t}) \in \mathbb{R}^{\mathrm{N}}$.

Demonstração: Provaremos apenas a primeira fórmula. Quanto à demonstração da segunda, basta observar que as contas continuam válidas trocando-se t por 0 nos locais onde deve-se fazer a troca para esse fim. 
Aqui será necessário considerar o operador $M_{k}$ tanto de acordo com sua definição usual como também agindo na variável de integração $\xi$ ao invés de $\chi$. Definimos

$$
M_{k}^{x}=\sum_{p=1}^{m} \mu_{k l}(x, t) \frac{\partial}{\partial x_{p}} \quad, \quad M_{k}^{\xi}=\sum_{p=1}^{m} \mu_{k l}(\xi, t) \frac{\partial}{\partial \xi_{p}}
$$

Observe que

$$
M_{k}^{x}\left\{Z_{j}(x, t)-Z_{j}(\xi, t)\right\}=-M_{k}^{\xi}\left\{Z_{j}(x, t)-Z_{j}(\xi, t)\right\}
$$

De fato,

$$
\delta_{k j}=M_{k}^{x} Z_{j}(x, t)=M_{k}^{x}\left\{Z_{j}(x, t)\right\}=M_{k}^{x}\left\{Z_{j}(x, t)-Z_{j}(\xi, t)\right\}
$$

porque $Z_{j}(\xi, t)$ independe de $x, e$

$$
-\delta_{k j}=-M_{k}^{\xi} Z_{j}(\xi, t)=M_{k}^{\xi}\left\{-Z_{j}(\xi, t)\right\}=M_{k}^{\xi}\left\{Z_{j}(x, t)-Z_{j}(\xi, t)\right\}
$$

porque $Z_{j}(x, t)$ independe de $\xi$. Logo, dada qualquer função holomorfa $F=F(\zeta), \zeta \in \mathbb{C}$, definindo-se $f(x, \xi, t)=F\left(Z_{j}(x, t)-Z_{j}(\xi, t)\right)$, temos

$$
M_{k}^{x}\{f(x, \xi, t)\}=-M_{k}^{\xi}\{f(x, \xi, t)\}
$$

Utilizaremos este fato com $F(\zeta)=e^{-\tau[\zeta]^{2}}$ nos cálculos abaixo:

$$
\begin{aligned}
M_{k}^{x} G_{\tau}(u)(x, t) & =\left(\frac{\tau}{\pi}\right)^{m / 2} \int_{\mathbb{R}^{m}} M_{k}^{x}\left\{e^{-\tau[Z(x, t)-Z(\xi, t)]^{2}}\right\} u(\xi, t) h(\xi) \operatorname{det} \frac{\partial Z}{\partial x}(\xi, t) d \xi \\
& =-\left(\frac{\tau}{\pi}\right)^{m / 2} \int_{\mathbb{R}^{m}} M_{k}^{\xi}\left\{e^{-\tau[Z(x, t)-Z(\xi, t)]^{2}}\right\} u(\xi, t) h(\xi) \operatorname{det} \frac{\partial Z}{\partial x}(\xi, t) d \xi \\
& =-\left(\frac{\tau}{\pi}\right)^{m / 2} \int_{\mathbb{R}^{m}} M_{k}^{\xi}\left\{e^{-\tau[Z(x, t)-Z(\xi, t)]^{2}}\right\} u(\xi, t) h(\xi) d Z(\xi, t) \\
& \stackrel{(4.21)}{=}\left(\frac{\tau}{\pi}\right)^{m / 2} \int_{\mathbb{R}^{m}} e^{-\tau[Z(x, t)-Z(\xi, t)]^{2}} M_{k}^{\xi}\{u(\xi, t) h(\xi)\} d Z(\xi, t) \\
& =\left(\frac{\tau}{\pi}\right)^{m / 2} \int_{\mathbb{R}^{m}} e^{-\tau[Z(x, t)-Z(\xi, t)]^{2}} M_{k}^{\xi}\{u(\xi, t)\} h(\xi) d Z(\xi, t)+ \\
& =\left(\frac{\tau}{\pi}\right)^{m / 2} \int_{\mathbb{R}^{m}} e^{-\tau[Z(x, t)-Z(\xi, t)]^{2}} M_{k}^{x} u(\xi, t) h(\xi) d Z(\xi, t)+ \\
& +\left(\frac{\tau}{\pi}\right)^{m / 2} \int_{\mathbb{R}^{m}} e^{-\tau[Z(x, t)-Z(\xi, t)]^{2}} u(\xi, t) M_{k}^{x} h(\xi) d Z(\xi, t) \\
& =G_{\tau}\left(M_{k}^{x} u\right)(x, t)+\left(\frac{\tau}{\pi}\right)^{m / 2} \int_{\mathbb{R}^{m}} e^{-\tau[Z(x, t)-Z(\xi, t)]^{2}} u(\xi, t) M_{k}^{x} h(\xi) d Z(\xi, t)
\end{aligned}
$$

Passando a primeira parcela ao primeiro membro obtemos a fórmula que queríamos demonstrar. 
Lema 4.23. As seguintes $n$ equações diferenciais valem em $\mathbb{R}^{\mathrm{N}}$ :

$$
\frac{\partial \operatorname{det} \frac{\partial Z}{\partial x}}{\partial t_{j}}+\sum_{k=1}^{m} \frac{\partial\left(\lambda_{j k} \operatorname{det} \frac{\partial Z}{\partial x}\right)}{\partial x_{k}}=0, \quad j=1, \ldots, n
$$

Demonstração: Seja $\varphi=\varphi(x, t)$ uma função teste arbitrária. Fixe $j=1, \ldots, n$ e defina a seguinte forma diferencial:

$$
\omega_{j}=d\left(\varphi d Z \wedge d t_{1} \wedge \cdots \wedge d t_{j-1} \wedge d t_{j+1} \wedge \cdots \wedge d t_{n}\right)
$$

onde

$$
\mathrm{dZ}=\mathrm{d} \mathrm{Z}_{1} \wedge \cdots \wedge \mathrm{d} \mathrm{Z}_{\mathrm{m}}=\operatorname{det} \frac{\partial \mathrm{Z}}{\partial x} \mathrm{dx}
$$

Sendo $\omega_{j}$ exata, temos $\int_{\mathbb{R}^{N}} \omega_{j}=0$. Mas por outro lado

$$
\begin{aligned}
w_{j} & =d \varphi d Z \wedge d t_{1} \wedge \cdots \wedge d t_{j-1} \wedge d t_{j+1} \wedge \cdots \wedge d t_{n}= \\
& =\left(\sum_{p=1}^{n} L_{p} \varphi d t_{p}+\sum_{k=1}^{m} M_{k} \varphi d Z_{k}\right) d Z \wedge d t_{1} \wedge \cdots \wedge d t_{j-1} \wedge \\
& =L_{j} \varphi d t_{j} \wedge d Z \wedge d t_{1} \wedge \cdots \wedge d t_{j-1} \wedge d t_{j+1} \wedge \cdots \wedge d t_{n}= \\
& =(-1)^{m+j-1} L_{j} \varphi d Z \wedge d t= \\
& =(-1)^{m+j-1}\left(L_{j} \varphi \operatorname{det} \frac{\partial Z}{\partial x}\right) d x d t
\end{aligned}
$$

Logo,

$$
\begin{aligned}
& 0= \int_{\mathbb{R}^{N}} \omega_{j}=(-1)^{m+j-1} \int_{\mathbb{R}^{N}}\left(L_{j} \varphi\right)(x, t) \operatorname{det} \frac{\partial Z}{\partial x}(x, t) d x d t \\
&=(-1)^{m+j-1} \int_{\mathbb{R}^{N}}\left(\frac{\partial \varphi}{\partial t_{j}}(x, t)+\sum_{k=1}^{m} \lambda_{j k}(x, t) \frac{\partial \varphi}{\partial x_{k}}(x, t)\right) \operatorname{det} \frac{\partial Z}{\partial x}(x, t) d x d t \\
&=(-1)^{m+j-1} \int_{\mathbb{R}^{N}} \frac{\partial \varphi}{\partial t_{j}}(x, t) \operatorname{det} \frac{\partial Z}{\partial x}(x, t) d x d t+ \\
& \quad+(-1)^{m+j-1} \sum_{k=1}^{m} \int_{\mathbb{R}^{N}} \lambda_{j k}(x, t) \frac{\partial \varphi}{\partial x_{k}}(x, t) \operatorname{det} \frac{\partial Z}{\partial x}(x, t) d x d t \\
&=(-1)^{m+j} \int_{\mathbb{R}^{N}} \varphi(x, t) \frac{\partial \operatorname{det} \frac{\partial Z}{\partial x}}{\partial t_{j}}(x, t) d x d t+ \\
& \quad+(-1)^{m+j} \sum_{k=1}^{m} \int_{\mathbb{R}^{N}} \varphi(x, t) \frac{\partial\left(\lambda_{j k} \operatorname{det} \frac{\partial Z}{\partial x}\right)}{\partial x_{k}}(x, t) d x d t \\
&=(-1)^{m+j} \int_{\mathbb{R}^{N}} \varphi(x, t)\left(\frac{\partial \operatorname{det} \frac{\partial Z}{\partial x}}{\partial t_{j}}(x, t)+\sum_{k=1}^{m} \frac{\partial\left(\lambda_{j k} \operatorname{det} \frac{\partial Z}{\partial x}\right)}{\partial x_{k}}(x, t)\right) d x d t
\end{aligned}
$$


Dada a arbitrariedade de $\varphi$ (que foi introduzida simplesmente para que $\omega_{j}$ fosse integrável), o resultado segue.

Lema 4.24. Se $u \in C^{\infty}\left(\mathbb{R}^{N}\right)$ é qualquer, então vale a fórmula:

$$
L_{j} G_{\tau}(u)(x, t)-G_{\tau}\left(L_{j} u\right)(x, t)=\left(\frac{\tau}{\pi}\right)^{m / 2} \int_{\mathbb{R}^{m}} e^{-\tau[Z(x, t)-Z(\xi, t)]^{2}} u(\xi, t) L_{j} h(\xi) \operatorname{det} \frac{\partial Z}{\partial x}(\xi, t) d \xi
$$

para todo $\mathrm{j}=1, \ldots, \mathrm{n}$ e todo $(x, \mathrm{t}) \in \mathbb{R}^{\mathrm{N}}$.

Demonstração: $\operatorname{Se} F=F(\zeta, t)$ é uma função holomorfa com relação a $\zeta \in \mathbb{C}$ e se definirmos $f(x, t)=F(Z(x, t), t)$, então:

$$
\begin{aligned}
& L_{j}\{f(x, t)\}= \frac{\partial}{\partial t_{j}}(F(Z(t, x), t))+\sum_{k=1}^{m} \lambda_{j k}(x, t) \frac{\partial}{\partial x_{k}}(F(Z(x, t), t))= \\
&= \sum_{l=1}^{m} \frac{\partial F}{\partial \zeta_{l}}(Z(x, t), t) \frac{\partial Z_{l}}{\partial t_{j}}(x, t)+\sum_{r=1}^{n} \frac{\partial F}{\partial t_{r}}(Z(x, t), t) \frac{\partial t_{r}}{\partial t_{j}}+ \\
& \quad \quad+\sum_{k=1}^{m} \lambda_{j k}(t, x) \sum_{l=1}^{m} \frac{\partial F}{\partial \zeta_{l}}(Z(x, t), t) \frac{\partial Z_{l}}{\partial x_{k}}(x, t)= \\
&=\sum_{l=1}^{m} \frac{\partial F}{\partial \zeta_{l}}(Z(x, t), t) L_{j} Z_{l}(x, t)+\frac{\partial F}{\partial t_{j}}(Z(x, t), t)= \\
&=\frac{\partial F}{\partial t_{j}}(Z(x, t), t)
\end{aligned}
$$

Utilizaremos este fato para a função holomorfa

$$
\tilde{G}_{\tau}(u)(\zeta, t)=\int_{\mathbb{R}^{m}} e_{\tau}(\zeta, \xi, \tau) u(\xi, t) h(\xi) \operatorname{det} \frac{\partial Z}{\partial x}(\xi, t) d \xi
$$

onde $e_{\tau}(\zeta, \xi, t)=e^{-\tau[\zeta-Z(\xi, t)]^{2}}$. Isto é, utilizaremos o fato que

$$
L_{j} G_{\tau}(u)(x, t)=\left(\frac{\tau}{\pi}\right)^{m / 2} L_{j} \tilde{G}_{\tau}(u)(Z(x, t), t)=\left(\frac{\tau}{\pi}\right)^{m / 2} \frac{\partial \tilde{G}_{\tau}(u)}{\partial t_{j}}(Z(x, t), t)
$$

Portanto devemos calcular $\partial_{t_{j}} \tilde{G}_{\tau}(u)$. No que se segue, denotaremos por $D_{x} Z$ a matriz de derivadas parciais $(\partial Z / \partial x)$. Como

$$
\tilde{G}_{\tau}(u)(\zeta, t)=\int e_{\tau}(\zeta, \xi, t) u(\xi, t) h(\xi) \operatorname{det} \frac{\partial Z}{\partial x}(\xi, t) d \xi=\int e_{\tau} u h \operatorname{det} D_{x} Z
$$


temos

$$
\begin{aligned}
\frac{\partial \tilde{G}_{\tau}(u)}{\partial t_{j}} & =\int\left(\frac{\partial\left(e_{\tau} u h\right)}{\partial t_{j}} \operatorname{det} D_{x} Z+e_{\tau} u h \frac{\partial \operatorname{det} D_{x} Z}{\partial t_{j}}\right) \\
& =\int\left\{\left(L_{j}\left(e_{\tau} u h\right)-\sum_{k=1}^{m} \lambda_{j k} \frac{\partial\left(e_{\tau} u h\right)}{\partial x_{k}}\right) \operatorname{det} D_{x} Z+e_{\tau} u h \frac{\partial \operatorname{det} D_{x} Z}{\partial t_{j}}\right\} \\
& =\int L_{j}\left(e_{\tau} u h\right) \operatorname{det} D_{x} Z-\int \sum_{k=1}^{m} \lambda_{j k} \frac{\partial\left(e_{\tau} u h\right)}{\partial x_{k}} \operatorname{det} D_{x} Z+\int e_{\tau} u h \frac{\partial \operatorname{det} D_{x} Z}{\partial t_{j}} \\
& =\int L_{j}\left(e_{\tau} u h\right) \operatorname{det} D_{x} Z+\int e_{\tau} u h \sum_{k=1}^{m} \frac{\partial\left(\lambda_{j k} \operatorname{det} D_{x} Z\right)}{\partial x_{k}}+\int e_{\tau} u h \frac{\partial \operatorname{det} D_{x} Z}{\partial t_{j}} \\
& =\int L_{j}\left(e_{\tau} u h\right) \operatorname{det} D_{x} Z+\int e_{\tau} u h\left(\frac{\partial \operatorname{det} D_{x} Z}{\partial t_{j}}+\sum_{k=1}^{m} \frac{\partial\left(\lambda_{j k} \operatorname{det} D_{x} Z\right)}{\partial x_{k}}\right) \\
& \stackrel{(4.23)}{=} \int L_{j}\left(e_{\tau} u h\right) \operatorname{det} D_{x} Z=\int L_{j}\left(e_{\tau}\right) u h \operatorname{det} D_{x} Z+ \\
& +\int e_{\tau} L_{j}(u) h \operatorname{det} D_{x} Z+\int e_{\tau} u L_{j}(h) \operatorname{det} D_{x} Z= \\
& =\int e_{\tau} L_{j}(u) h \operatorname{det} D_{x} Z+\int e_{\tau} u L_{j}(h) \operatorname{det} D_{x} Z
\end{aligned}
$$

Para a última passagem, note que $\mathrm{L}_{\mathbf{j}}\left(e_{\tau}\right)=\mathrm{L}_{\mathbf{j}}\left\{\exp \left(-\tau[Z(x, t)-Z(\xi, t)]^{2}\right)\right\}=0$. Ficamos então com

$$
\begin{aligned}
L_{j} G_{\tau}(u)(x, t)=\left(\frac{\tau}{\pi}\right)^{m / 2} \int_{\mathbb{R}^{m}} e^{-\tau[Z(x, t)-Z(\xi, t)]^{2}} L_{j} u(\xi, t) h(\xi) \operatorname{det} \frac{\partial Z}{\partial x}(\xi, t) d \xi+ \\
\quad+\left(\frac{\tau}{\pi}\right)^{m / 2} \int_{\mathbb{R}^{m}} e^{-\tau[Z(x, t)-Z(\xi, t)]^{2}} u(\xi, t) L_{j} h(\xi) \operatorname{det} \frac{\partial Z}{\partial x}(\xi, t) d \xi
\end{aligned}
$$

que é a fórmula enunciada.

Corolário 4.25. A sequência de polinômios em Z obtida com a Fórmula de Aproximação de Baouendi-Trèves converge à solução correspondente em norma $\mathrm{C}^{\infty}$.

Demonstração: Seja $u \in C^{\infty}(W)$ uma solução de $\mathcal{V}$, como no teorema 4.17. Já sabemos que, qualquer que seja $h \in C^{\infty}\left(\mathbb{R}^{m}\right)$ suportada em $\{|x| \leq R\}$ e valendo 1 em $\{|x| \leq R / 2\}$,

$$
\mathrm{G}_{\tau}(\mathrm{u}), \mathrm{E}_{\tau}(\mathrm{u}) \underset{\tau \rightarrow \infty}{\longrightarrow} \mathrm{hu}
$$

uniformemente em $\{|x| \leq R / 4\} \times\{|t| \leq T\}$, e essa convergência se dá uniformemente com relação a u também. Seja $k=1, \ldots, m$ qualquer. Como $M_{k} u$ é solução de $\mathcal{V}$ (visto que os campos $L_{j}$ e $M_{k}$ comutam), concluímos que também

$$
\mathrm{G}_{\tau}\left(M_{\mathrm{k}} \mathrm{u}\right), \mathrm{E}_{\tau}\left(\mathrm{M}_{\mathrm{k}} \mathrm{u}\right) \underset{\tau \rightarrow \infty}{\longrightarrow} h M_{k} \mathrm{u}
$$


uniformemente em $\{|x| \leq R / 4\} \times\{|t| \leq T\}$. Por outro lado, provamos no lema 4.22 que a expressão para $M_{k} G_{\tau}(u)-G_{\tau}\left(M_{k} u\right)$ é a mesma que a expressão para $G_{\tau}$, apenas trocandose $h$ por $M_{k} h$. Esta última é também uma função suave suportada em $\{|x| \leq R\}$, então também temos

$$
M_{k} G_{\tau}(u)-G_{\tau}\left(M_{k} u\right) \underset{\tau \rightarrow \infty}{\longrightarrow}\left(M_{k} h\right) u
$$

Mas em $\mathrm{U}$, sendo $\mathrm{h} \equiv 1$, temos $M_{k} h=0$, e assim

$$
M_{k} G_{\tau}(u)=G_{\tau}\left(M_{k} u\right)+\left(M_{k} G_{\tau}(u)-G_{\tau}\left(M_{k} u\right)\right) \underset{\tau \rightarrow \infty}{\longrightarrow} h M_{k} u+\left(M_{k} h\right) u=M_{k} u
$$

uniformemente. Analogamente provamos que

$$
M_{k} E_{\tau}(u) \underset{\tau \rightarrow \infty}{\longrightarrow} M_{k} u \quad, \quad L_{j} G_{\tau}(u) \underset{\tau \rightarrow \infty}{\longrightarrow} L_{j} u \quad, \quad L_{j} E_{\tau}(u) \underset{\tau \rightarrow \infty}{\longrightarrow} L_{j} u
$$

uniformemente em $\mathrm{U}$, para todos $j=1, \ldots, n, k=1, \ldots, \mathrm{m}$ (o lema 4.22 também dá conta da primeira convergência, e o lema 4.24 prova a segunda, enquanto que a última é trivial, pois os dois lados são zero).

Agora basta lembrarmos que toda derivada de ordem 1 é uma combinação $\mathrm{C}^{\infty}$-linear dos $L_{j}$ e $M_{k}, j=1, \ldots, n, k=1, \ldots, m$, pois estes campos geram o espaço tangente. Dessa forma, se D é uma derivação de primeira ordem, então

$$
\mathrm{DG}_{\tau}(\mathrm{u}) \underset{\tau \rightarrow \infty}{\longrightarrow} \mathrm{Du}, \mathrm{DE}_{\tau}(\mathrm{u}) \underset{\tau \rightarrow \infty}{\longrightarrow} \mathrm{Du}
$$

uniformemente em $U$. Iterando-se esse resultado, temos que $G_{\tau}(u)$ e $E_{\tau}(u)$ convergem a $u$ em $U$ na topologia $C^{\infty}$. Examinando-se a prova do teorema 4.17, vemos que isso é tudo o que precisávamos para concluir o mesmo da sequência de polinômios em Z obtida ali. 



\section{Referências Bibliográficas}

[BT] Baouendi, M. e Trèves, F., A local constancy principle for the solutions of certain overdetermined systems of first-order linear partial differential equations,. Adv. in Math. Suppl. Stud. 7. Academic Press, New York-London (1981), 245-262.

[BZ] Bergamasco, A. e Zani, S., Global analytic regularity for structures of co-rank one. Comm. Partial Differential Equations 33 (2008), 933-941.

[BCH] Berhanu, S., Cordaro, P. e Hounie, J., An Introduction to Involutive Structures. New Mathematical Monographs 6. Cambridge University Press, Cambridge (2008).

[C] Cartan, H., Variétés analytiques réelles et variétés analytiques complexes. Bull. Soc. Math. France 85 (1957), 77-99.

[F1] Folland, G., Introduction to Partial Differential Equations, $2^{\mathrm{a}}$ edição. Princeton University Press, Princeton (1995).

[F2] Folland, G., Real Analysis: Modern Techniques and Their Applications, $2^{\mathrm{a}}$ edição. Pure and Applied Mathematics: A Wiley Series of Texts, Monographs and Tracts 40. Wiley (1999).

[G] Grauert, H., On Levi's problem and the imbedding of real-analytic manifolds. Ann. of Math. (N.2) 68 (1958), 460-472.

[Gr] Greenfield, S.J., Hypoelliptic vector fields and continued fractions. Proc. Amer. Math. Soc. 31 (1972), 115-118.

[H] Hörmander, L., The Analysis of Linear Partial Differential Operators I: Distribution Theory and Fourier Analysis, $2^{\mathrm{a}}$ edição. Grundlehren der mathematischen Wissenschaften 256, Springer-Verlag (1990). 
[J] John, F., Partial Differential Equations, $4^{\mathrm{a}}$ edição. Applied Mathematical Sciences 1, Springer-Verlag (1982).

[L] Lewy, H. An example of a smooth linear partial differential equation without solution. Ann. of Math. 66 (1957), 155-158.

[NN] Newlander, A., Nirenberg, L. Complex coordinates in almost complex manifolds. Ann. of Math. 65 (1957), 391-404.

[R] de Rham, G., Variétés Différentiables: Formes, Courants, Formes Harmoniques. Actualites Scientifiques et Industrielles. Hermann (1955).

[S] Saks, S., Theory of the Integral, $2^{a}$ edição revisada. Dover Books on Mathematics. Dover Publications (2005).

[T1] Trèves, F., Approximation and Representation of Functions and Distributions Annihilated by a System of Complex Vector Fields. Centre de mathématiques, École polytechnique (1981).

[T2] Trèves, F., Hypo-Analytic Structures: Local Theory. Princeton University Press, Princeton (1993).

[T3] Trèves, F., Remarks about certain first-order linear PDE in two variables. Comm. Partial Differential Equations 5(4) (1980), 381-425. 